\title{
MODELLING AND PREDICTION OF BLOCKAGE EFFECTS ON THE OPERATION OF TIDAL TURBINES
}

KOH WEI XIANG MARTIN

\section{Interdisciplinary Graduate School Energy Research Institute@ NTU (ERI@N)}

A thesis submitted to the Nanyang Technological

University in partial fulfilment of the requirement for the degree of

Doctor of Philosophy 


\section{Statement of Originality}

I hereby certify that the work embodied in this thesis is the result of original research and has not been submitted for a higher degree to any other University or Institution.

$20 / 12 / 2016$

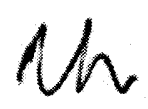

Date

Koh Wei Xiang Martin 


\section{Acknowledgements}

I would like to thank the following people for their help and support in the completion of this thesis.

First, I would like to thank my parents for their support in all that I do.

Next, I would like to thank my supervisors and members of my Thesis Advisory Committee for their input and guidance during the dissertation process. My main supervisor, Dr Ng Yin Kwee Eddie, for all the technical advice and questions raised in the duration of my work. My co-supervisor, Dr Lim Siow Yong, for helping me clarify my thoughts and showing me new perspectives on my work. Dr Narasimalu Srikanth, for his valuable feedback on the relevance of my work for industrial applications. Also included is Dr Michael Lochinvar S. Abundo from the Energy Research Institute@NTU, who has provided me with valuable help and guidance.

I would also like to thank my friends in the office for their emotional support and making the dissertation process more enjoyable, especially Aw Kuan Tai, William Toh, Tan Chun Kiat, Koh Jian Hao, Ken Tay and Chew Kok Hon.

I am also grateful to the Interdisciplinary Graduate School for providing financial support during the dissertation process, as well as for the many programs and activities planned to provide us with a more well rounded education.

Lastly, I would like to thank God, without whom none would be possible. 
Table of Contents

$\begin{array}{lr}\text { Acknowledgements } & 1\end{array}$

Table of Contents 3

$\begin{array}{lr}\text { Table Captions } & 9\end{array}$

$\begin{array}{ll}\text { Figure Captions } & 11\end{array}$

$\begin{array}{lr}\text { Abbreviations } & 19\end{array}$

$\begin{array}{ll}\text { Abstract } & 22\end{array}$

$\begin{array}{lll}\text { Chapter } 1 & \text { Introduction } & \mathbf{2 5}\end{array}$

$1.1 \quad$ Background . . . . . . . . . . . . . . 26

1.2 Problem statement . . . . . . . . . . . . . 27

1.3 Objective and scope ................ 27

1.4 Thesis overview . . . . . . . . . . . . . 28

1.5 Contributions . . . . . . . . . . . . . . 29

$1.6 \quad$ Limitations . . . . . . . . . . . . . . . . . . 29

$\begin{array}{lll}\text { Chapter } 2 & \text { Literature review } & 31\end{array}$

2.1 Introduction . . . . . . . . . . . . . . 32

2.2 Turbine modeling methods . . . . . . . . . . . . 32

2.2.1 Blade Element Momentum theory (BEMT) . . . . . . . . 32

2.2 .1 .1 Airfoil data . . . . . . . . . . . . . . . . 33

2.2 .1 .2 Tip loss correction . . . . . . . . . . . . . . . 34

2.2.1.3 High induction correction . . . . . . . . . . . 36

2.2.2 Computational Fluid Dynamics (CFD) . . . . . . . . 37

2.2.2.1 Actuator methods . . . . . . . . . . . . . . . . . . 37

2.2.2.2 Resolving the physical geometry . . . . . . . . . . . 39 
2.3 Blockage modeling and correction . . . . . . . . . 40

2.4 Wake studies . . . . . . . . . . . . . . . . . 42

2.5 Concluding remarks ................. 44

Chapter $3 \quad$ Analytical turbine models 45

3.1 Linear momentum actuator disc theory (LMADT) . . . . . 46

$3.2 \quad$ BEMT . . . . . . . . . . . . . . . . . . 48

3.2 .1 Tip loss correction . . . . . . . . . . . . . 50

3.2.1.1 Prandtl's tip loss correction model . . . . . . . . . . . . 50

3.2.1.2 Goldstein's tip loss correction model . . . . . . . . . . . 51

3.2.1.3 Shen's tip correction model . . . . . . . . . . . . . 52

3.3 Blockage correction models . . . . . . . . . . . . . 53

3.4 Concluding remarks . . . . . . . . . . . . . 55

$\begin{array}{lll}\text { Chapter } 4 & \text { CFD model } & 57\end{array}$

$4.1 \quad$ Methodology . . . . . . . . . . . . . . . . 58

$4.2 \quad$ Numerical Model . . . . . . . . . . . . . . . . 59

4.2.1 Turbulence representation . . . . . . . . . . . . 61

4.2 .2 Actuator disk model . . . . . . . . . . . . . . . . 62

4.3 Validation ................... 64

4.4 Discussion ....................... 69

Chapter 5 Effects of Reynolds number and tip loss models on the accuracy of BEMT $\quad 71$

5.1 Introduction . . . . . . . . . . . . 72

$5.2 \quad$ Rotor models . . . . . . . . . . . . . . . 72

5.3 Methodology . . . . . . . . . . . . . 73

$5.4 \quad$ Results and Discussion . . . . . . . . . . . 78

5.4.1 Effect of Reynolds number variation . . . . . . . . . . 78

5.4.2 Effect of tip loss functions . . . . . . . . . . . . . 85

$5.5 \quad$ Concluding remarks . . . . . . . . . . . . . . 88 
Chapter 6 CFD study of the performance and wake recovery of a single tidal turbine under various flow $\begin{array}{ll}\text { and blockage conditions } & 91\end{array}$

6.1 Introduction . . . . . . . . . . . . . . . . . . 92

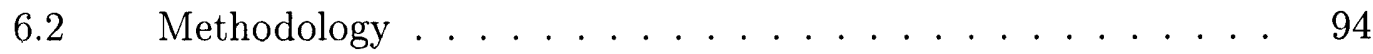

6.3 Results and discussion . . . . . . . . . . . . . . 95

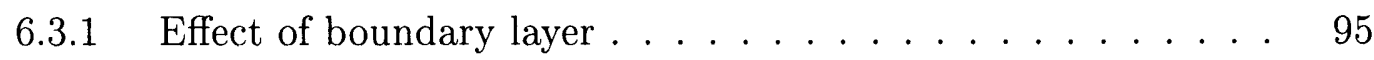

6.3 .2 Effect of flow properties . . . . . . . . . . . . . . 102

6.3.3 Effect of channel geometry . . . . . . . . . . . . . . 105

6.3 .4 Wake recovery . . . . . . . . . . . . . . . . . . 109

6.3 .5 Discussion . . . . . . . . . . . . . . . . . . 112

6.4 Concluding remarks $\ldots \ldots \ldots \ldots \ldots \ldots$

Chapter 7 Analytical prediction of the performance and wake recovery of a single tidal turbine under blocked conditions 117

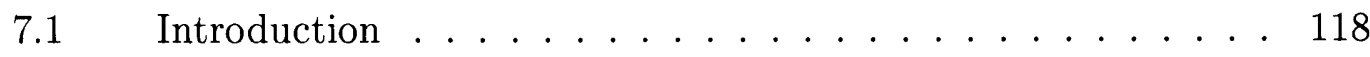

7.2 Analytical models . . . . . . . . . . . . . . . . . 119

7.2.1 Blockage correction models . . . . . . . . . . . . . . . . 119

7.2 .2 Wake models . . . . . . . . . . . . . . . . . 120

7.3 Methodology . . . . . . . . . . . . . . . 122

7.4 Results and discussion . . . . . . . . . . . . . . . . . 123

7.4 .1 Performance prediction . . . . . . . . . . . . . 123

7.4 .2 Wake prediction . . . . . . . . . . . . 128

$7.5 \quad$ Concluding remarks . . . . . . . . . . . . . . . . 129

Chapter 8 Modelling and prediction of the effect of blockage on the performance of a 3 bladed rotor 133

8.1 Introduction . . . . . . . . . . . . . . . 134

$8.2 \quad$ CFD model . . . . . . . . . . . . . . . . . . . . 134

8.3 Validation . . . . . . . . . . . . . . 136

8.4 Methodology . . . . . . . . . . . . . . . . 137

$8.5 \quad \operatorname{Results} \ldots \ldots \ldots \ldots \ldots \ldots$ 
8.5.1 CFD modeling . . . . . . . . . . . . . . . . . 139

8.5.2 BEM prediction . . . . . . . . . . . . . 140

8.5 .3 Discussion . . . . . . . . . . . . . . . . . . . 142

8.6 Concluding remarks . . . . . . . . . . . . . . 143

Chapter 9 Performance and wake recovery of a tidal turbine array under various blockage conditions $\quad 145$

9.1 Introduction . . . . . . . . . . . . . . . . 146

9.2 Methodology . . . . . . . . . . . . . . 146

9.3 Results . . . . . . . . . . . . . . . 149

9.3.1 Array performance . . . . . . . . . . . . . . 149

9.3 .2 Wake study . . . . . . . . . . . . . . 155

9.3 .3 Discussion . . . . . . . . . . . . . . . 158

9.4 Concluding remarks ................. 161

Chapter 10 Conclusion 163

10.1 Summary . . . . . . . . . . . . . . . . . 164

10.2 Implications . . . . . . . . . . . . . . . . 167

$10.3 \quad$ Future works . . . . . . . . . . . . . . . 168

$\begin{array}{ll}\text { References } & 170\end{array}$

$\begin{array}{ll}\text { References } & 171\end{array}$

Chapter A Validation cases contour plots $\quad 185$

A.1 Contour plots for the empty flume . . . . . . . . . . . . 185

A.2 Contour plots for a turbine in the flume . . . . . . . . . 187

A.3 Contour plots for turbines with different added turbulence intensity ......................... 189

$\begin{array}{lll}\text { Chapter B } & \text { Contour plots for single turbine cases } & 191\end{array}$

B.1 Plots for a single turbine in channels with the boundary layer present . . . . . . . . . . . . . . . 192

B.2 Plots for a single turbine in channels without the boundary layer 195

B.3 Plots for a single turbine in channels with different depth . . 198 
$\begin{array}{lll}\text { Chapter C Velocity ratios for single turbine case } & 201\end{array}$

Chapter D MATLAB code for blockage correction 203

$\begin{array}{ll}\text { Related publications } & 205\end{array}$ 


\section{Table Captions}

Table 4.1 Grid convergence index of the three levels of mesh created 60

Table 5.1 Blade sectional data for the turbine used by Bahaj et al. $[1] \ldots \ldots \ldots \ldots 76$

Table 5.2 Blade sectional data for the turbine used by Mycek et al. $[2] \ldots \ldots \ldots \ldots 77$

Table 6.1 Cross sectional dimensions and reference velocities of the channels tested . . . . . . . . . . . . 94

Table 6.2 Cross-sectional dimensions of the additional channels introduced . . . . . . . . . . . . . 106

Table 6.3 Channels and the respective variables involved in each data set . . . . . . . . . . . . 106

Table 7.1 Cross sectional dimensions of the channels tested . . . . 122

Table 8.1 Size of the different meshes and results for the convergence test . . . . . . . . . . . . . 135

Table 8.2 Details of the channels used to study the blockage effect 137

Table 9.1 Values of the global and local blockage ratios used in CFD simulations . . . . . . . . . . . . . . 148

Table C.1 Table of velocity ratios for channels without boundary layer . . . . . . . . . . . . . . 201

Table C.2 Table of velocity ratios for channels with boundary layer 201 


\section{Figure Captions}

Figure 1.1 An example of a turbine that is being used for the MeyGen project [3] . . . . . . . . . . . . 26

Figure 3.1 Illustration of the 1D linear momentum actuator disk theory ................ . . 4 46

Figure 3.2 Illustration of the forces on a turbine blade and changes in flow through a turbine [4] . . . . . . . . . 48

Figure 3.3 Illustration of a turbine being placed in a bounded flow [5] 53

Figure 4.1 Cross section of the medium mesh . . . . . . . . . 60

Figure 4.2 The velocity $(\mathrm{a}, \mathrm{b})$ and turbulence intensity $(\mathrm{c}, \mathrm{d})$ profiles of the flume generated by the different turbulence models. . . . . . . . . . . . . . 65

Figure 4.3 The a) centreline velocity and b) turbulence profiles of an actuator disk generated by the different turbulence models. . . . . . . . . . . . . . .

Figure 4.4 Predicted vertical velocity profile for various turbulence models compared to experimental results at various downstream locations

solid line: ske, dotted line: rke, dashed line: skw, dotteddashed line: kw-sst . . . . . . . . . . . . 66 66

Figure 4.5 Predicted vertical turbulence intensity profile for various turbulence models compared to experimental results at various downstream locations

solid line: ske, dotted line: rke, dashed line: skw, dotteddashed line: kw-sst . . . . . . . . . . 66

Figure 4.6 The a) centreline velocity and b) turbulence profiles of an actuator disk generated by different amount added turbulence at the disk . . . . . . . . . . 67 
Figure 4.7 Predicted vertical velocity profile for various amounts of turbulence added at the disk compared to experimental results at various downstream locations

No added turbulence: dotted line, $\triangle I_{m}$ added: solid line, $2 \triangle I_{m}$ added: dashed line . . . . . . . . . . . 68

Figure 4.8 Predicted vertical turbulence intensity profile for various amounts of turbulence added at the disk compared to experimental results at various downstream locations No added turbulence: dotted line, $\triangle I_{m}$ added: solid line, $2 \triangle I_{m}$ added: dashed line . . . . . . . . . . .

Figure 4.9 Comparison of the wake generated by the momentum sink approach and the porous media formulation . . 70

Figure 5.1 Comparison of the a) lift and b) drag coefficients predicted by XFoil and experiments for the NACA 63-815 airfoil $[6] \ldots \ldots \ldots \ldots \ldots$

Figure 5.2 Variation of lift and drag coefficients with Reynolds number as predicted by XFOIL for NACA 63-815 used in the turbine by Bahaj et al. [1] . . . . . . . . . . .

Figure 5.3 Variation of lift and drag coefficients with Reynolds number for NACA 63-418 used in the turbine by Mycek et al. $[2] \ldots \ldots \ldots \ldots \ldots \ldots \ldots$

Figure 5.4 Variation of L/D ratio with Reynolds number for a) NACA 63-815 and b) NACA 63-418 . . . . . . . .

Figure 5.5 Effect of using the local Reynolds number on the predicted power coefficient of the turbine by Bahaj et al. [1] for turbine pitch angles of 20,25 and 27 degrees respect-

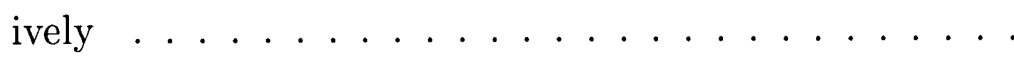

Figure 5.6 Effect of using the local Reynolds number on the predicted thrust coefficient of the turbine by Bahaj et al. [1] for turbine pitch angles of 20,25 and 27 degrees re-

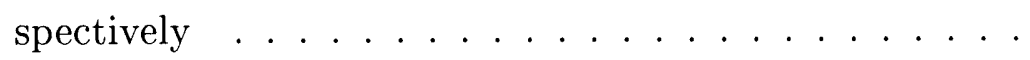


Figure 5.7 Variation of Reynolds number experienced by the turbine blade used by Bahaj et al. [1] across the whole operational range of TSRs for turbine pitch angles of 20 , 25 and 27 degrees respectively . . . . . . . . .

Figure 5.8 Effect of using the local Reynolds number on the induction factors of the turbine by Bahaj et al. [1], taken at a pitch angle of 20 degrees with a TSR of $6 \ldots . .$.

Figure 5.9 Effect of using the local Reynolds number on the angle of attack on the blade of the turbine by Bahaj et al. [1], taken at a pitch angle of 20 degrees with a TSR of 6 . .

Figure 5.10 Effect of using the local Reynolds number on the predicted power and thrust coefficient of the turbine by Mycek et al. [2] . . . . . . . . . . . . . .

Figure 5.11 Effect of using the local Reynolds number on the induction factors of the turbine by Mycek et al. [2], taken at a TSR of $5 \ldots \ldots \ldots \ldots$

Figure 5.12 Variation of Reynolds number for the turbine by Mycek et al. [2] for various TSRs . . . . . . . . . . . .

Figure 5.13 Effect of Shen's correction on the predicted power coefficient of the turbine by Bahaj et al. [1] for turbine pitch angles of 20, 25 and 27 degrees respectively . . . . . .

Figure 5.14 Effect of Shen's correction on the predicted thrust coefficient of the turbine by Bahaj et al. [1] for turbine pitch angles of 20, 25 and 27 degrees respectively . . . . . .

Figure 5.15 Effect of Shen's correction on the induction factors of the turbine by Bahaj et al. [1] for a TSR of 6 and blade pitch angle of 20 degrees . . . . . . . . . . .

Figure 5.16 Effect of Shen's correction on the angle of attack for a TSR of 6 and blade pitch angle of 20 degrees . . . . . .

Figure 5.17 Effect of Shen's correction on the axial force coefficient at a TSR of 6 for a blade pitch angle of 20 degrees . . .

Figure 5.18 Effect of Shen's correction on the tangential force coefficient at a TSR of 6 for a blade pitch angle of 20 degrees 
Figure 6.1 Velocity profiles for all the channels at a) 5 diameters before and b) 11 diameters after the turbine location compared with experimental data $[7] \ldots 96$

Figure 6.2 Turbulence Intensity profiles for all the channels at a) 5 diameters before and b) 11 diameters after the turbine location compared with experimental data $[7] \ldots .$. .

Figure 6.3 Comparison between $C_{T, 0}$ and $C_{T, E}$ for all channels a) in the presence of and $b$ ) without the presence of the boundary layer . . . . . . . . . . . . . .

Figure 6.4 $C_{T, E} / C_{T, 0}$ for all channels a) in the presence of and b) without the presence of the boundary layer . . . . . .

Figure 6.5 Comparison of the velocity ratios of the different channels a) in the presence of and b) without the presence of the boundary layer . . . . . . . . . . . . .

Figure 6.6 Difference in velocity ratios measured with and without the presence of a boundary layer . . . . . . . . .

Figure 6.7 Difference in a) thrust and b) power extracted by a turbine due to the presence of the boundary layer . . . . . 100

Figure 6.8 Comparison of the differences in velocity ratios with and without the boundary layer for channels of a) varying blockage ratios, b) varying aspect ratios . . . . . . . . 101

Figure 6.9 Difference in thrust and power generated by a turbine due to the presence of the boundary layer for channels of a) varying blockage ratios, b) varying aspect ratios . 102

Figure 6.10 Changes of the a) velocity ratio, b) maximum velocity drop downstream of the turbine due to variation in the inlet velocity taken at thrust coefficient of $1.0 \ldots 103$

Figure 6.11 Changes of the a) velocity ratio, b) maximum velocity drop downstream of the turbine due to variation in the Turbulence Intensity taken at thrust coefficient of 1.0 . 104

Figure 6.12 Interaction effects between Turbulence Intensity and velocity taken at thrust coefficient of $1.0 \ldots 105$ 
Figure 6.13 Effect of varying the aspect ratio of the channel on the measured velocity ratio taken at thrust coefficient of $1.0 \quad 108$

Figure 6.14 Effect of varying the depth of the channel on the measured velocity ratio taken at thrust coefficient of $1.0 \ldots 108$

Figure 6.15 Effect of varying the blockage ratio of the channel on the measured velocity ratio taken at thrust coefficient of 1.0108

Figure 6.16 Comparison of the effects of dissipation on the velocity ratio with respect to aspect ratio variations . . . . . 109

Figure 6.17 Comparison of the decay rate of turbulence intensity in the channels of different aspect ratios . . . . . . . 109

Figure 6.18 Comparison of the wake generated in channels of constant depth and varying area for a) the full length of the domain, b) from 5D onwards, c) various downstream locations . . . . . . . . . . . . . 110

Figure 6.19 Comparison of the wake generated in channels of constant area and different depth for a) the full length of the domain, b) from 5D onwards, c) various downstream locations ................... 111

Figure 7.1 Comparison of the different analytical models with CFD simulations for different thrust coefficients. CFD-1 refers to the data set taken from Chapter 6, CFD-2 refers to the simulations performed with lower inlet turbulent in-

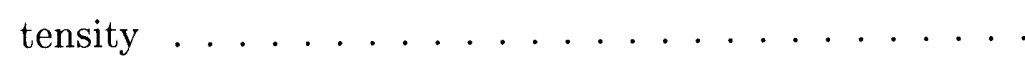

Figure 7.2 Variation of the velocity ratio predicted by the different models with increasing blockage ratio for thrust coefficients of $0.8,0.9$ and 1.0 respectively . . . . . . 125

Figure 7.3 Variation of the maximum velocity reduction predicted by the different models with increasing blockage ratio for thrust coefficients of $0.8,0.9$ and 1.0 respectively . . . 125

Figure 7.4 Velocity ratio contour plot of the mid plane of Channel 0126

Figure 7.5 Velocity plot at $0.8 \mathrm{D}$ downstream of the turbine plane . 126 
Figure 7.6 Turbulent Kinetic Energy plot at $0.8 \mathrm{D}$ downstream of the turbine plane . . . . . . . . . . 126

Figure 7.7 Thrust coefficients predicted by the different models compared with experimental data from McTavish et al. [8] . 127

Figure 7.8 Wake recovery for the different channels predicted with CFD compared with the modified Jensen wake model and the modified wake model of Bastankhah and PortéAgel [9] . . . . . . . . . . . . . .

Figure 8.1 Cross section of the mesh around a turbine blade from Mycek et al. [2] . . . . . . . . . . . . . 135

Figure 8.2 Validation of the CFD model with results obtained with different turbulence models . . . . . . . . . . 136

Figure 8.3 Flowchart illustrating the process to predict the blocked performance of a turbine using BEM and a blockage prediction model . . . . . . . . . . . . . 138

Figure 8.4 a) Power and b) Thrust coefficients predicted by CFD in the different channels . . . . . . . . . . . . . . . . . 139

Figure 8.5 Comparison between the power and thrust coefficients predicted by CFD and BEM for a) channel B0, b) channel B1, c) channel B2 . . . . . . . . . . . . 140

Figure 8.6 Comparison between the a) power coefficients and b) thrust coefficients with (solid lines) and without (dashed lines) the Reynolds number effect . . . . . . . . . . 141

Figure 9.1 Variation of the velocity ratio with changing blockage ratio for a single turbine taken from Chapter 6 . . . . 147

Figure 9.2 Schematic of the array of turbines in a channel . . . 147

Figure 9.3 Velocity ratio experienced by each turbine in the array compared with that experienced by a single turbine at the same global and local blockage ratio for different thrust coefficients . . . . . . . . . . . . . . . . . . . . 149

Figure 9.4 Velocity profile along the centreline of the array for various local blockage at global blockage G1 . . . . . . . 149 
Figure 9.5 Variation of the calculated equivalent single turbine blockage with the global blockage ratio for the different local blockage ratios . . . . . . . . . . . . 151

Figure 9.6 Variation of the calculated equivalent single turbine blockage with the local blockage ratio for the different global blockage ratios . . . . . . . . . . . . . 151

Figure 9.7 Comparison of the predictions obtained by the overall best fit equation with results obtained by CFD simulations for arrays with a local blockage of $0.4 \ldots 152$

Figure 9.8 Comparisons of predictions obtained by alternative fittings with the original fitting and results obtained from CFD simulations for arrays with local blockage ratio of 0.4153

Figure 9.9 Comparison of the new fitting with the original fitting and results obtained from CFD simulations for arrays with local blockage ratio of $0.4 \ldots \ldots$. . . . . . 153

Figure 9.10 Comparisons of the average velocity ratio experienced by each turbine between that predicted using the equivalent single turbine blockage obtained from equation 9.5 and that obtained by CFD simulations for an array operating at a thrust coefficient of $0.7 \ldots \ldots \ldots 154$

Figure 9.11 Comparisons between the equivalent single turbine blockage obtained by the different methods of varying the global blockage ratio and that predicted by equation 9.5154

Figure 9.12 Velocity profile along the centreline of the array for various global blockage at local blockage L2 . . . . . . . . 156

Figure 9.13 Lateral velocity profile for various global blockage ratios for the local blockage L2 taken at 20D downstream of the turbine plane . . . . . . . . . . . 157

Figure 9.14 Turbulent kinetic energy profile for various global blockage ratios for the local blockage L2 taken at 20D downstream of the turbine plane . . . . . . . . . 157 
Figure 9.15 a) Lateral velocity profile and b) turbulent kinetic energy for arrays of various sizes for the local blockage L2 taken at 20D downstream of the turbine plane . . . . . . 157

Figure 9.16 Comparison between the centreline wake recovery of the arrays a) G1L2, b) G1L3 with the wake of a single turbine at their respective global and local blockage ratio . 158

Figure 9.17 Comparison of the wake recovery predicted by different methods of varying the global blockage ratio at 20D downstream of the turbine location . . . . . . . . 159

Figure A.1 Velocity plots by the different turbulence models for the empty channel . . . . . . . . . . . . . . 185

Figure A.2 Turbulence Intensity plots by the different turbulence models for the empty channel . . . . . . . . . . . 186

Figure A.3 Pressure plots by the different turbulence models for the empty channel . . . . . . . . . . . . . . . 186

Figure A.4 Velocity plots by the different turbulence models for a turbine in the channel . . . . . . . . . . . . 187

Figure A.5 Turbulence Intensity plots by the different turbulence models for a turbine in the channel . . . . . . . . . 188

Figure A.6 Pressure plots by the different turbulence models for a turbine in the channel . . . . . . . . . . . . . . 188

Figure A.7 Velocity plots for different levels of turbulence added by the turbine . . . . . . . . . . . . . . . . . . 189

Figure A.8 Turbulence Intensity plots for different levels of turbulence added by the turbine . . . . . . . . . . . . 190

Figure A.9 Pressure plots for different levels of turbulence added by the turbine . . . . . . . . . . . . . . . 190

Figure B.1 Velocity plots for the different channels with the boundary layer present . . . . . . . . . . . . . . . 192

Figure B.2 Turbulence Intensity plots for the different channels with the boundary layer present . . . . . . . . . . . . . 193 
Figure B.3 Pressure plots for the different channels with the boundary layer present . . . . . . . . . . . . . . . 194

Figure B.4 Velocity plots for the different channels without the boundary layer . . . . . . . . . . . . . . . . 195

Figure B.5 Turbulence Intensity plots for the different channels without the boundary layer . . . . . . . . . . . 196

Figure B.6 Pressure plots for the different channels without the boundary layer . . . . . . . . . . . . . . . . . 197

Figure B.7 Velocity contours for channels with varying depth for turbulent length scale varying with depth (a-d), constant turbulent length scale $(\mathrm{e}-\mathrm{h}) \ldots \ldots \ldots$

Figure B.8 Turbulence Intensity contours for channels with varying depth for turbulent length scale varying with depth (ad), constant turbulent length scale (e-h) . . . . . . . 199

Figure B.9 Pressure contours for channels with varying depth for turbulent length scale varying with depth (a-d), constant turbulent length scale $(\mathrm{e}-\mathrm{h}) \ldots \ldots \ldots$ 


\section{Abbreviations}

AOA Angle of Attack

AD Actuator Disk

BE Blade Element

BEMT Blade Element Momentum theory

CFD Computational Fluid Dynamics

RANS Reynolds Averaged Navier Stokes

LES Large Eddy Simulation

TSR Tip Speed Ratio 


\section{Abstract}

A series of works have been performed to better understand the effects of channel blockage on the performance and wake recovery of tidal turbines. The prediction of the performance of tidal turbines operating under blocked conditions has also been studied.

Computational fluid dynamics (CFD) studies of a single turbine operating under various flow conditions and channel sizes revealed that the effect of blockage is to cause the turbine to experience a higher velocity than the free-stream velocity. The boundary layer is also found to greatly affect the prediction of performance of a tidal turbine, and neglecting its presence would lead to the performance of the tidal turbine being overestimated. Channel depth is also found to affect the performance of the tidal turbine. Increasing the blockage ratio of the channel is found to increase the recovery of the far wake, while the near wake was minimally affected.

Predictions of performance by analytical models were found to be more sensitive to changes in blockage ratios when compared to that predicted from CFD. Nevertheless, there is generally good agreement between the predictions made by analytical models and CFD for typical operating ranges of thrust coefficients. Prediction of the wake recovery was also compared. When modified to work under blockage conditions, predictions of the wake recovery by analytical models had good agreement with the wake predicted by CFD. The model used by Bahaj et al. in 2007 to predict the effect of blockage on the performance on a turbine, together with the Jensen wake model, is recommended for predicting the effects of blockage on the performance and wake recovery of a single turbine.

Coupling the blockage prediction model by Bahaj et al. with a BEM model allowed the prediction of the performance of a 3 bladed rotor under blockage 
conditions with a good degree of accuracy. In addition, Reynolds number effects are found to be important in accurately predicting the performance of the turbine under blocked conditions due to the increase in experienced velocity. Including the Reynolds number effect results in the optimum rotation speed and power coefficient increasing as the blockage ratio increases as compared to when it is not included.

The performance and wake recovery of a small array of fixed size under various blockage scenarios was also studied. Performance of each turbine within the array is found to be between that of a single turbine operating with the same global blockage and local blockage as the array. The equivalent single turbine blockage is defined as the blockage ratio required for a single turbine to produce the same performance as the turbine within the array. The equivalent single turbine blockage is used to characterise the performance of the turbine in the array, and an expression was developed to describe it.

Wake recovery of the array was studied as well. Increasing global blockage ratios was found to increase the wake recovery of the array, while locating turbines too close to each other lead to their wakes merging and taking longer to recover. When there is no significant wake interaction between the wakes of the individual turbines in the array, the wakes were found to be similar to that of a single turbine operating with the same global blockage ratio as the turbine. The minimum inter-turbine spacing for this is found to be two turbine diameters. 


\section{Chapter 1}

\section{Introduction}

It is important to consider the effect of blockage on the operation of tidal turbines. However, current methods of modelling the effect of blockage are very resource consuming. As such, the use of analytical models would be very attractive in predicting the effects of blockage on tidal turbine operations. This thesis explores the validation and use of analytical models to predict the performance of tidal turbines under various modes of operation. A brief summary of the main contributions and limitations of the thesis is also presented. 


\subsection{Background}

As the tidal turbine technology matures and the industry advances, turbines are increasingly being deployed in arrays in order to harvest energy more efficiently. It is important to be able to predict the performance of the array before it is deployed, and much work has been done on this aspect. The operation of tidal turbines and wind turbines are very similar, and many prediction tools currently used on tidal turbines have been adopted from the wind turbine industry. However, the operating environment of a wind turbine is very different from that of a tidal turbine. Besides the difference in working fluid, tidal turbines also operate in blocked conditions, where the turbine is located close to the fluid boundaries. For example, the MeyGen project in Pentland Firth involves placing turbines with rotor diameters of $18 \mathrm{~m}$ in waters with an average depth of $35 \mathrm{~m}$ [10], of which an example can be seen in figure 1.1. This will have an effect on the performance of the turbines, which is still not entirely understood.

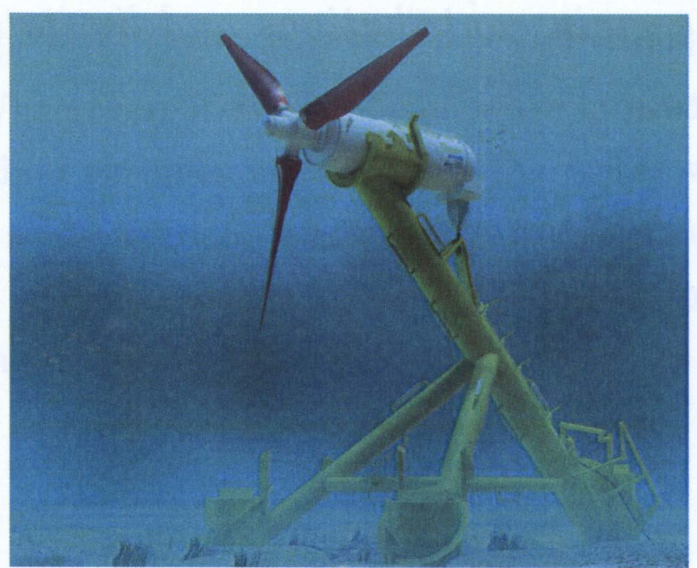

Figure 1.1: An example of a turbine that is being used for the MeyGen project [3]

Furthermore, certain countries may require a certain portion of a channel to be unoccupied for other reasons. This may change the nature of the resource that is measured in the absence of turbines. Completely occupying the channel may also impede the flow of water through the channel, causing 
less energy to be generated than what is predicted.

Modelling the effects of energy extraction on the regional flow patterns are commonly carried out using coastal area models. However, such models require an accurate input of the forces that the turbines exert on the flow. Computational fluid dynamics (CFD) is able to study the effects of blockage on the local flows around the turbine, and the resulting performance of the turbine as a result of those local flows. However, CFD would be very expensive to use in the initial planning of a tidal turbine array. Analytical models exists for predicting the performance of the turbine under blockage conditions, but their assumptions do not often match with real world conditions. Thus, application of the models to real world data without understanding the differences would lead to inaccurate results. However, understanding of the differences between the model assumptions and real world conditions is lacking in available literature found to date. Also, the accuracy of the models are often unknown as no validation has been found to be performed.

\subsection{Problem statement}

Understanding of the effects of channel blockage on the performance and wake recovery of tidal turbines is still lacking. Accuracy and implementation of analytical models to predict the effects of channel blockage is also not well studied.

\subsection{Objective and scope}

The objective of this thesis is to provide a better understanding of the effects of channel blockage on the performance and wake recovery of tidal turbines through the use of CFD. Differences between real world conditions simulated through CFD and analytical models will be explored and quantified. In addition, effects of blockage on the performance and wake recovery of an 
array are to be investigated. Application of analytical models to predict the performance and wake recovery of an array will also be explored.

\subsection{Thesis overview}

This thesis is divided into the following chapters.

Chapter 2 presents a literature review of the different different methods for modelling the tidal turbine, as well as the effects of blockage on the operation of the tidal turbine.

Chapter 3 introduces the different analytical models commonly used to predict the performance of a tidal turbine, while Chapter 4 introduces the CFD model that will be used throughout the thesis.

Chapter 5 presents a study investigating the effects of several factors on the accuracy of the BEM model. This is done by comparing the outputs of the BEM model with experimental results.

A CFD simulation of the performance and wake recovery of a single turbine under different flow conditions and channel geometries is presented in Chapter 6 . Differences between real world conditions and assumptions made by analytical models will also be investigated and quantified in this chapter.

Chapter 7 presents an evaluation of different blockage correction models by comparing them against CFD simulations performed in Chapter 6. Wake prediction models are also evaluated against the wake produced from $\mathrm{CFD}$ simulations.

Chapter 8 combines the results in Chapter 5 and 7 to produce an analytical model of a turbine capable of predicting the performance of a physical turbine under blocked conditions.

Chapter 9 presents a study on the performance and wake recovery of a tidal turbine array that is subjected to various blockage cases. 
Lastly, Chapter 10 presents a summary of the findings from the entire thesis, as well as possible extensions to the works presented in this thesis.

\subsection{Contributions}

This research lead to several outcomes.

1. A method for quantifying the effect of blockage on the performance of a turbine.

2. Quantification of the difference in predicted power and thrust produced by a turbine when the effect of boundary layer is neglected.

3. An understanding of how the channel geometry affects the performance of a tidal turbine.

4. Validation of the different analytical models with CFD simulations, and recommendation of suitable models to be used.

5. Development of an expression to determine the blockage experienced by a turbine within a small array of fixed size subjected to various blockage scenarios.

6. An understanding of how the wake recovery of a tidal turbine is affected by channel blockage.

\subsection{Limitations}

Some limitations of the research presented in this thesis are listed below and will be addressed as future works.

1. Only constant forcing for simulations is considered in this thesis.

2. Only rectangular channels are considered in this thesis. 
3. The study on arrays is only for a single row. Thus, application to multi-row arrays is not yet verified.

4. The array studied is of a fixed size of 5 turbines. Hence, the expression to determine blockage would not be accurate for arrays of other sizes. 


\section{Chapter 2}

\section{Literature review}

This chapter aims to provide an overview of the available literature on concepts and models that are used in this thesis. Techniques for modeling tidal turbines will first be discussed, followed by blockage effects and wake studies. It is found that the accuracy of analytical models used to predict the blockage effect is currently unknown. Furthermore, there are no comparisons between the different models found in literature. As such, part of the aims of this thesis include determining the accuracy of the different analytical models used to predict the blockage effect. 


\subsection{Introduction}

Most of the models and concepts used in tidal turbines are adapted from the wind turbine field. As the tidal turbine field is relatively young compared to the wind turbine area, there would be more literature available for similar concepts in the wind turbine field. As such, where literature related to tidal turbines are found lacking, those related to wind turbines would be brought in.

\subsection{Turbine modeling methods}

\subsubsection{Blade Element Momentum theory (BEMT)}

As more tidal turbine designs reach maturity and prepare for deployment, it is necessary to be able to predict the performance of the turbine under various operating conditions. The BEMT model is a very popular tool for rotor design and analysis, as it requires little computing power compared to Computational Fluid Dynamics (CFD). Thus, results can be obtained much quicker.

The BEMT model is a combination of the Blade Element theory (BE) and the Momentum theory. The BE theory involves dividing the rotor blade into many elements, and the rotor's thrust and torque profiles are modelled from the lift and drag characteristics of each blade element. Meanwhile, the momentum theory considers the linear and angular momentum changes across the rotor. By combining the 2 theories, momentum changes in the fluid are forced to match with the blade forces at each blade element $[11,12]$. It was first developed to study aircraft and marine propellers, but has been adapted to study wind turbines. Recently, it has also been shown to be applicable to tidal turbines as well [13], with studies showing that the BEMT model matching experimental results with reasonable accuracy $[13,14,4]$.

The accuracy of the BEMT model depends on the reliability of the many 
sub-models that are contained within it.

\subsubsection{Airfoil data}

A key assumption of the BEMT model is that there are no interactions between the different blade elements. However, experiments have shown that there is radial flow along the blade caused by the blade rotation. Thus, the accuracy of the BEMT solution might be affected if the 2D aerodynamic force coefficients are used without any correction. This effect was first recorded by Himmelskamp [15] in his study of propellers, and was subsequently investigated by many authors $[16,17,18,19]$. Current understanding of the effects on the flow brought about by blade rotation is described in the following paragraphs.

Due to centrifugal effects caused by blade rotation, the boundary layer is pushed outwards radially, leading it to become thinner and more stable compared to non-rotating state. Also, centrifugal effects on separated flow at the trailing edge causes radial flow along the blade. As the centrifugal effects increase radially, pressure in the separated flow decreases and contributes to the normal force on the turbine blade $[16,18,19]$. This effect is known as "Centrifugal pumping". Also, additional negative pressure in the separated flow results in larger negative pressure gradient in the chord-wise direction, increasing the stability of the boundary layer. Subsequently, this results in requiring a larger AOA for the airfoil to stall. This effect is known as "Stall Delay" [16]. All these effects lead to increased lift in the affected areas.

However, the outcome of rotational effects on the drag-coefficient is still not conclusive, with different researchers arguing for the increase [15] and decrease [17] of drag as a result of blade rotation.

Radial flow caused by centrifugal effects also prevents the formation of negative pressures near the blade tip, leading to reduced force coefficients compared to non-rotating case [16]. 
A number of analytical models has been introduced to correct for this effect. Snel et al. [20] first proposed a correction for the rotational effects based on the analysis of boundary layer equations. This was further extended by Du and Selig [17], and Lindenburg [21]. Bak et al. [22] proposed a model based on pressure measurements from wind tunnel experiments. Corrigan and Schilling [23] also developed a correction based on boundary layer analysis and helicopter experimental data. This was later used for wind turbine applications by Tangler and Selig [24].

A number of analytical models to correct for the rotational effects were compared with experimental and CFD results. CFD was found to produce the best prediction of the results obtained from experiments. The comparison also highlighted the difficulties for analytical models to consistently capture the flow physics found in a rotating airfoil [25]. Yu et al. [26] used CFD to study the stall delay phenomenon. The correction by Du and Selig [17] was also used to correct a lifting surface code. The correction improved the prediction accuracy of power production by lifting surface code, but was not able to capture the complete range of stall delay effects along the blade.

Dowler and Schmitz [27] noted that most analytical correction models work by modifying the $2 \mathrm{D}$ airfoil before it is used by the BEMT process. This assumes that rotational effects being corrected for is similar across all blades, and that phenomena specific to certain blades might be ignored. A new approach to correcting for rotational effects was then introduced, where the correction is dependent on parameters calculated during the process of solving the BEMT equations. Results show that there is good match between the predicted and experimental data.

\subsubsection{Tip loss correction}

The tip loss factor can be interpreted in a number of ways.

The BEMT model assumes that the forces on the rotor are averaged over each annular stream-tube, meaning that all fluid particles passing through 
the rotor disk at a particular radial position would experience the same loss in axial momentum. Whereas in reality, spaces exist between the blades in a real turbine where the influence of the rotor blades are not felt. This means that the average drop in momentum at a particular radial position will be lower than that predicted by the BEMT model. This effect is more pronounced towards the edge of the rotor disk, as there are more empty spaces at the tip compared to the root. Thus, the momentum drop averaged over each annulus that a fluid particle experienced in a real turbine would be much lower that that predicted by BEMT alone [11].

Another way of interpreting this would be to consider the circulation around each blade. Using the circulation theory, Betz arrived with a solution that described the wake produced by an optimal rotor as one that is rigid [28]. Some assumptions for the solution include having an infinite number of blades, and that the rotor is lightly loaded. Prandtl improved the solution by introducing a factor to account for a finite number of blades. The assumption made is that the vortex structures in a wake are similar to flow around flat plates placed in the wake of a rotor [16, 28], although this is only true at high tip speeds ratios [16]. Also based on Betz's work on the optimal rotor, Goldstein proposed a more accurate solution of the flow in a rotor wake based on Bessel functions [16, 29]. It is noted that the agreement between solution provided by Prandtl and Goldstein improves with the number of rotor blades. Glauert [30] later modified Prandtl's correction to act on the axial momentum, which was used in $[31,32]$ to derive their tip loss models.

Shen et al. analysed Glauert's model and found that an outcome of the model is that the relative axial velocity at the tip is zero, which means that the vortex generated by the tip is unable to be convected into the wake. Since the models by Wilson and Lissaman [31] and De Vries [32] were based on Glauert's model, they would also lead to the same outcome. Further analysis using the Blade Element theory found that the force at the blade tips is generally not zero. However, this is not the case physically due to the equalisation of pressure at the tip. Thus, a new tip loss model that modified the force coefficients in addition to the induced velocities was introduced 
[33].

Studies were done to compare the performance of the various tip loss models against results obtained from CFD [34, 35, 36, 37]. Hansen and Johansen [34] performed a numerical experiment of a wind turbine and compared the power produced by the numerical model to that predicted by the standard BEMT model, as well as Shen's tip loss model. It was found that all three models predicted similar radial loadings on the turbine blade. Lee et al. [35] performed CFD experiments with a tidal turbine. There was good agreement around the design tip speed ratio (TSR) between the power coefficient predicted by CFD and that by the BEMT model. However, there was bad agreement at high and low TSRs, which was attributed to rotational flow effects not accounted for in their BEMT model.

Sankar [36] compared the behaviour of the tip loss function between that calculated from CFD and BEMT utilising the Prandtl tip loss function. Differences between the two methods were attributed to vortex stretching, and an improvement based on Prandtl's model was introduced. Similarly, Madsen et al. [37] compared BEMT to CFD simulations of an actuator disk (AD) model, and introduced corrections to the BEMT model to correct for pressure variation in the rotating wake and wake expansion.

Branlard and Dixon [38] noted that Prandtl and Goldstein's code did not account for a number of wake phenomena, and introduced a new model based on a vortex code and used with a look-up table. Masters and Chapman [39] reported that iterative methods to solve the BEMT equations do not work well under off-design conditions. A new implementation based on combined Monte Carlo and sequential quadratic optimisation was introduced. Used together with Prandtl's model for tip and hub corrections, the new model showed good agreement when compared with a commercial software.

\subsubsection{High induction correction}

Glauert [40] presented an empirical equation relating the axial induction 
factors with experimental measurements of thrust at high axial induction factors, where the momentum theory breaks down. The axial induction factor is a measure of how much the velocity of the flow has reduced as it passes through the turbine. A mathematical definition can be found in Chapter 3. Buhl Jr [41] noted that a discontinuity appears between the momentum curve and Glauert's empirical curve when the tip loss function is applied. A new equation that includes the tip loss factor was derived using algebraic means, and was subsequently used in Chapman et al. [42].

\subsubsection{Computational Fluid Dynamics (CFD)}

\subsubsection{Actuator methods}

Actuator methods refer to the use of parameters to describe the turbine. Three types of actuator methods are currently used to model wind and tidal turbines. The first method is the actuator disk (AD) method, in which the swept area of the turbine blades is represented by a thin disk, where changes is momentum of the flow takes place. The turbine is parameterised by the thrust coefficient of the turbine. The second method is the actuator line method, where the turbine blades are represented by lines on which the aerodynamic forces of the blades act. The lift and drag coefficients of the different blade sections are thus used to parametrise the turbine. The third method is the actuator surface method, where the turbine blades are represented by the pressures experienced by the turbine blades.

Among the three actuator methods, the AD method is the most widely used model to represent tidal turbines because of its simplicity $[43,44,45,7,46$, 47,48 ]. It has also been shown to be reliable in predicting the far wake of a turbine when compared to experiments [43, 7]. Harrison et al. [43] noted that the AD model alone does not produce the small scale turbulence that is generated by experimental porous disks, unless turbulent sources are defined at the disk location as well. Blackmore et al. [7] showed that the inclusion of turbulent sources at the disk location had an effect on the wake recovery of 
the disk. In order to compare well to experimental porous disk experiments, a turbulent length scale with the size of the holes in the porous disk was found to provide the best match.

Harrison et al. [49] also noted that the AD method alone cannot model a turbine in detail as it does not include information on the blades, and that fully resolving the turbine and the wake region would be too computationally expensive. Instead of using a constant source term defined by the thrust coefficient of the turbine, Harrison et al. [49] used the blade element actuator disk (AD-BE) method, where source terms within the AD domain were determined using the blade element approach. This resulted in improved modeling of the near wake region compared to the uniform $\mathrm{AD}$ method. Turnock et al. [50] uses the BEMT theory to determine the strength of the sources, and uses it to model an array of turbines. Various other authors have also used the previously mentioned methods of using the blade element method or BEMT to determine the strength of the source terms $[51,52$, $53,54,55,56]$. Batten et al. [44] compared the uniform AD model with the $\mathrm{AD}-\mathrm{BE}$ model, and found that the AD-BE model was preferable to the uniform $\mathrm{AD}$ model as it produces a better wake prediction and allows the calculation of turbine performance.

Due to the fact that the computational requirements for the $\mathrm{AD}$ and and $\mathrm{AD}$ BEM models are significantly less than fully resolving the turbine geometry, they are often used to perform simulations on turbine arrays [46, 50, 51, $55,56,57]$. The AD-BEMT model was also used to study the effects of different velocity on wake recovery of the turbine $[53,54]$, while Nishino and Willden [48] applied the uniform AD model to determine the effects of different channel blockage on wake recovery.

Schluntz and Willden [58] used the actuator line method to study a single turbine, while Churchfield et al. [59] used the actuator line method to simulate an array of tidal turbines. Andersen et al. [60] also used the actuator line method to study a vertical axis turbine. There is no known study that used the actuator surface to model a tidal turbine. 


\subsubsection{Resolving the physical geometry}

There are a number of simulation studies that resolved the physical geometry of the turbine $[35,61,62,63,64,65,66,67,68]$. Most of the studies were focused on predicting the power and thrust of the turbine due to the relative abundance of experimental power and thrust data available, and the availability established methodologies to predict the power and thrust measurements in the absence of experimental data. The turbine by Bahaj et al. [1] was modelled using different turbulence models [61, 62, 66]. LES was found to produce the best match between predicted and experimental power and thrust coefficients, while the $k-\omega$ SST model produced satisfactory agreement with a significantly reduced computational cost. Lee et al. [35] compared the predictions of BEMT with the predictions obtained from CFD, and found that they had good agreement around the design tip speed ratio.

Conversely, there are few studies for the wake of the turbine, possibly due to the lack of published wake data at the time the studies were done. As such, no conclusions about the accuracy can be made. Afgan et al. [66] compares the wake produced by LES and RANS simulations. LES was found to produce vortices that could not be captured completely by RANS simulations. Turbulent kinetic energy in the wake was also lower in RANS simulations compared to LES. Jo et al. [65] presented the wake generated by their turbine using the SST turbulence model and compared to the Jensen semi-empirical wake model. Good agreement was reported between the far wake predicted by CFD and that predicted by the Jensen model.

Masters et al. [51] compared the AD-BEM, BEMT and the resolving of the physical geometry to experimental results, and concluded that all three methods could agree with each other only if blockage correction is performed consistently among all the models. 


\subsection{Blockage modeling and correction}

Blockage theory for tidal turbines are based on the theories developed to correct for the interference between the wind tunnel walls and propeller in wind tunnel experiments. This was then adapted for use by wind turbines, and subsequently tidal turbines.

Bramwell et al. [69] first noticed that the wind tunnel walls had an interference effect on the behaviour of the propeller, and introduced an empirical expression relating the equivalent free-stream airspeed to the observed airspeed in the wind tunnel. Wood and Harris [70] later derived a theoretical expression to predict the equivalent free-stream airspeed. This was later simplified by Glauert [71] by representing the propeller with an actuator disk.

Although the model by Glauert [71] was developed to correct propeller data, it has also been used to correct wind turbine data [72]. Barnsley and Wellicome [73] modified Glauert's model [71] to for use in wind turbine applications, and Bahaj et al. [1] used it to correct data from a tidal turbine experiment. This was subsequently used by other authors correcting data obtained from water tunnel experiments $[61,8,74]$.

Maskell [75] proposed that the increase in force experienced by a bluff body in a wind tunnel is due to the body experiencing the higher dynamic pressure of the bypass flow caused by the tunnel walls. Whelan et al. [76] used this theory to correct their data obtained from water tunnel experiments. They also modified the actuator disk model to account for the variation in water surface due to the drop in pressure in the wake of the turbine.

There are many studies that investigated the effects of blockage on various aspects of the turbine operation. Nishino and Willden [48], Kolekar and Banerjee [68], McTavish et al. [8], Birjandi et al. [77] studied the effects of blockage on the performance of the turbine. Kolekar and Banerjee [68] investigated the effect of blockage on power produced by a turbine, and found that increasing the blockage ratio of the channel beyond $10 \%$ resulted in 
an increase in power production. It was also reported that power production at low tip speed ratios did not improve with increasing blockage as additional power produced due to the blockage effect was offset by power loss due to stall on the blades. Utilising the model by Whelan et al. [76], Birjandi et al. [77] evaluated the effect of blockage on a vertical axis turbine by varying the water level in the water tunnel, and found that the power coefficient increased as blockage increased while the turbine was fully submerged. McTavish et al. [8] studied the effect of blockage on the thrust coefficient of the turbine by having turbines of different diameters in the same channel. It was revealed that while the uncorrected thrust coefficients increased with increasing blockage, correction for blockage effects resulted in the thrust coefficients becoming almost constant for all blockage ratios.

The effect of blockage on a turbine's wake was also studied. McTavish et al. [8] studied the wake of a turbine by injecting dye into the water just upstream of the blade tip location, allowing the wake development to be studied visually. It was found that the wake width was reduced by half when the blockage ratio increased from $6.3 \%$ to $25.4 \%$. Vortex pairing was also observed to be delayed as the blockage ratio increased.

Chen and Liou [78], Medici et al. [79] studied the effect of blockage on the upstream velocity of wind tunnels experiments. Chen and Liou [78] quantified the blockage effect with the model used by Bahaj et al. [1], and found that blockage effects are largely influenced by the TSR, blade pitch angle of the turbine and blockage ratio of the tunnel. Medici et al. [79] found that velocities were affected by more than 3 diameters upstream of the turbine.

Investigations of blockage effects in wind farms was found to largely mirror that of tidal turbine farms. Experiments on tidal turbines $[80,56]$ have shown that the flow between the turbine is accelerated by having small inter-turbine distances between two laterally arranged turbines. The optimal inter-turbine distance to produce this effect have been found to be approximately 3 diameters measured from the turbine centres. Turbines 
placed in this region of accelerated flow subsequently experienced higher rates of power production. This findings were subsequently replicated in studies of wind farms $[81,82]$.

Models discussed previously mainly dealt with correcting turbine data for the interference from tunnel walls. However, tidal turbines are likely to be placed in environments where blockage effects are inevitable. As such, it is important to know how the turbine performance will differ from the data obtained from water tunnel experiments when placed in a different blocked environment. Garrett and Cummins [5] used the actuator disk theory to develop a model of a tidal turbine operating in bounded flow to predict the change in turbine performance due to blockage. Results showed that the turbine's performance coefficients increased with increasing blockage ratios. This was later adapted and used to develop models for a partial tidal fence $[83,84]$, and also a wind farm [85]. Houlsby et al. [86] also developed a model based on the $\mathrm{AD}$ theory, but modified it to account for the free-surface. A search through literature also yielded work in [87], who essentially developed the same model as Garrett and Cummins [5], but for wind turbines.

\subsection{Wake studies}

The wake produced by a turbine has been subjected to intense study as it affects the downstream positioning of additional turbines, with both physical turbines $[64,88,89,90,91,92,93,94,95,2,96]$ and actuator disks $[43,45$, $7,50,53,54,1,91,97,98]$ being equally popular in generating the wake. Although both devices are fundamentally different, Bahaj and Myers [91] concluded from their experimental studies that their wakes are similar in the far wake given similar flow conditions.

The wake can generally be divided into the near wake and the far wake. The near wake is the portion of the wake that contains complex flow fields which are determined by the geometry of the turbine [99]. In experimental studies of the near wake of a tidal turbine, Chamorro et al. [88] identified significant 
tangential velocity near the blade root, as well as radial velocities near the tip. Tip vortices were also present up till 2 diameters downstream. The far wake is the region where flow recovery takes place due to wake mixing, and where the geometry of the turbine is not a dominating factor. The transition point between the near and far wake is still ambiguous. Sanderse [99] defines the transition point as when the shear layer reaches the wake axis, while $[100,101]$ define it as when vortices from the turbine break down. This point is typically within 5 diameters of the rotor plane for wind turbines $[99,100]$. However, Tedds et al. [90] defined it as up to 7 diameters in their experimental study of a tidal turbine. Since the near wake is typically highly turbulent, it is not advisable to locate another turbine within the near wake as it will shorten the turbine's fatigue life, in addition to reduced power capture. As such, there is much interest in the factors affecting the recovery of the far wake.

Various factors affecting the wake of a turbine have been identified from the many experimental and computational wake studies performed. Thrust produced by the turbine is found to mainly affect the near wake and has little effect on the far wake [43, 91, 97]. However, Myers and Bahaj [95] also discovered that the support structure of the turbine also affects the near wake when its wake interacts with the wake generated by the turbine. Far wake recovery is found to be driven by the mixing of the wake with the freestream flow [97]. Thus, the turbulent intensity of the ambient flow would have a great impact on the far wake recovery $[43,91,94,2]$, as the level of wake mixing is proportional to the amount of turbulence in the flow.

Other flow factors also found to affect the wake recovery are the turbulent length scale and the flow velocity. In CFD simulations of actuator disks, larger turbulent length scales were found to result in increased velocity deficit closer to the turbine while increasing the rate of wake recovery at the same time. Increased length scales have also lead to wider wake widths [45]. Wake length and width was found to increase with increasing flow velocity [54], while recovery is faster when the turbine is subjected to accelerating flows [53]. 
Being in close proximity to channel boundaries have also been found to affect the wake recovery of a turbine. Bahaj et al. [1] discovered that the free surface and channel bed serves to restrain the vertical wake expansion, while $[97,98]$ found that the wake recovers slower when the turbine is near the channel bed due to the presence of the boundary layer. Although there is an increase in turbulence in the boundary layer, velocity is also slower. This would lead to lower levels of wake mixing, leading to the wake recovery being slower than if the turbine is located higher in the water column. Placing the turbine too close to the surface would lead to a drop in the free surface and skewing of the wake [68].

\subsection{Concluding remarks}

It is seen from literature that BEMT and CFD based models are established methods to model the performance of the turbine.

Studies have shown how turbine performance increases with increasing blockage ratios, but none of them provide a comparison to the predictions made by existing correction models. This can be a source of frustration to modellers who are trying to model turbines without using CFD, as the accuracy of the blockage correction models is currently not known. There is also a lack of blockage studies on tidal turbine arrays, which will be important as the technology advances. 


\section{Chapter 3}

\section{Analytical turbine models}

Accurately modeling the performance of a tidal turbine is a very expensive process, either numerically or experimentally. Fortunately, mathematical models have been developed to predict the performance of a turbine by making certain assumptions. One such assumption is that the fluid is considered inviscid, thus viscous flow effects like flow mixing and the boundary layer are ignored. This chapter introduces the different mathematical models that are commonly used to model the various aspects of tidal turbine operation and discussed in Chapter 2. 


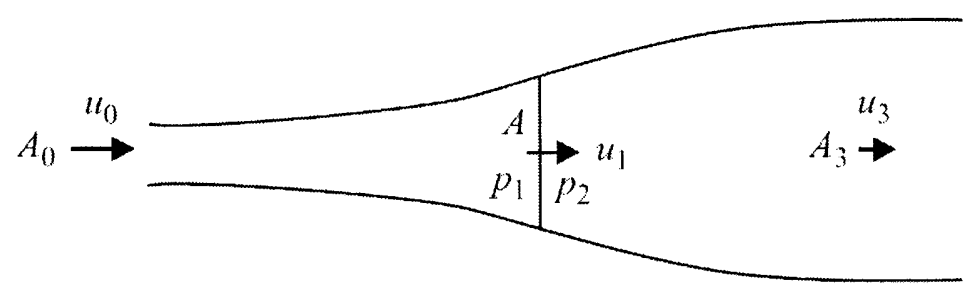

Figure 3.1: Illustration of the 1D linear momentum actuator disk theory

\subsection{Linear momentum actuator disc theory (LMADT)}

The 1D linear momentum actuator disk is a simplified representation of a device adding or extracting energy from a $1 \mathrm{D}$ inviscid flow, and it is used by many other models describing various aspects of tidal turbines. Energy extraction is assumed to occur across an infinitely thin membrane. The effects of flow rotation caused by the spinning turbine are ignored.

The theory is illustrated in figure 3.1 for the case of a tidal turbine with swept area, $A$, extracting energy from a tidal flow in an infinite domain. The following equations give the derivation of the thrust and power coefficients that are widely used to describe the performance of tidal turbines.

Considering the flow inside the stream-tube, applying the conservation of mass would yield equation 3.1, while applying the conservation of momentum at both ends of the stream-tube results in equation 3.2.

$$
\begin{aligned}
& u_{0} A_{0}=u_{2} A=u_{3} A_{3} \\
& T=\rho u_{0}^{2} A_{0}-\rho u_{3}^{2} A_{3}
\end{aligned}
$$

where $T$ represents the thrust produced by the turbine.

Applying Bernoulli's equation to the stream-tube results in equations 3.3 
and 3.4.

$$
\begin{aligned}
& p_{0}+\frac{1}{2} \rho u_{0}^{2}=p_{1}+\frac{1}{2} \rho u_{2}^{2} \\
& p_{3}+\frac{1}{2} \rho u_{3}^{2}=p_{2}+\frac{1}{2} \rho u_{2}^{2}
\end{aligned}
$$

Subtracting equation 3.4 from equation 3.3 and inserting $A$ results in equation 3.5.

$$
\frac{1}{2} \rho A u_{0}^{2}\left[1-\frac{u_{3}^{2}}{u_{0}^{2}}\right]=A\left(p_{1}-p_{2}\right)=T
$$

Equating equation 3.5 to equation 3.2 yields the following relation.

$$
u_{2}=\frac{1}{2}\left(u_{0}+u_{3}\right)
$$

The axial induction factor, $a$, is introduced and is defined in equation 3.7. It is a measure of the drop of the velocity through the turbine compared to the free-stream velocity.

$$
a=\frac{u_{0}-u_{2}}{u_{0}}
$$

The velocity through the turbine and the wake velocity can thus be expressed by the following equations.

$$
\begin{aligned}
& \frac{u_{2}}{u_{0}}=1-a \\
& \frac{u_{3}}{u_{0}}=1-2 a
\end{aligned}
$$




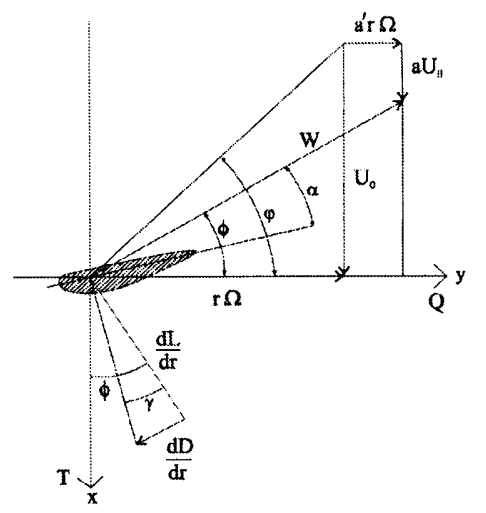

Figure 3.2: Illustration of the forces on a turbine blade and changes in flow through a turbine [4]

The coefficients of thrust and power according to the LMADT can thus be expressed in equations 3.10 and 3.11 respectively.

$$
\begin{gathered}
C_{T}=\frac{T}{\frac{1}{2} \rho A u_{0}^{2}}=4 a(1-a) \\
C_{P}=\frac{T u_{1}}{\frac{1}{2} \rho A u_{0}^{3}}=4 a(1-a)^{2}
\end{gathered}
$$

\subsection{BEMT}

Figure 3.2 shows the forces on the turbine blade, and changes in the flow as it passes through the turbine. By combining the blade element theory and the momentum theory, momentum changes in the fluid are forced to match with the blade forces at each blade element $[11,12]$. The following equations are used in the BEM formulation as found in [4]. Similar equations with their derivation can be found in wind energy textbooks and guides $[11,12,102]$. Symbols used in the equations represent the same quantities as shown in Figure 3.2.

Equations 3.12 and 3.13 represent the thrust and torque of the rotor as a 
result of axial and rotational momentum changes of the fluid through the rotor.

$$
\begin{gathered}
\frac{d T}{d r}=4 \pi r \rho U_{0}^{2} a(1-a) F \\
\frac{d Q}{d r}=4 \pi r^{3} \rho U_{0} \Omega a^{\prime}(1-a) F
\end{gathered}
$$

where $F$ represents the tip loss model to be used.

Equations 3.14 and 3.15 define the expression for thrust and torque of the rotor as obtained using the blade element theory.

$$
\begin{aligned}
& \frac{d T}{d r}=\frac{1}{2} \rho c B W^{2}\left(C_{l} \cos \phi+C_{d} \sin \phi\right) \\
& \frac{d Q}{d r}=\frac{1}{2} \rho c B W^{2}\left(C_{l} \sin \phi-C_{d} \cos \phi\right)
\end{aligned}
$$

where $B$ represents the number of blades in the turbine.

After obtaining the expression for $W$ in terms of $U$, and equating equations 3.12 to 3.14 , and equations 3.13 to 3.15 , equations 3.16 and 3.17 for $a$ and $a^{\prime}$ are obtained respectively.

$$
\begin{aligned}
\frac{a}{1-a} & =\frac{\sigma\left(C_{l} \cos \phi+C_{d} \sin \phi\right)}{4 F \cdot \sin ^{2} \phi} \\
\frac{a^{\prime}}{1-a} & =\frac{\sigma\left(C_{l} \sin \phi-C_{d} \cos \phi\right)}{4 F \cdot \lambda_{r} \cdot \sin ^{2} \phi}
\end{aligned}
$$

where solidity ratio $\sigma=\frac{c B}{2 \pi r}$, and $\lambda_{r}$ represents the local sectional tip speed ratio, not to be confused with the global tip speed ratio. These equations are then solved by iterating $\phi$. 
Blade sectional thrust and power coefficients are thus solved to result in equations 3.18 and 3.19 .

$$
\begin{gathered}
\frac{d C_{T}}{d x}=\frac{2 \sigma F(1-a)^{2}\left(C_{l} \cos \phi+C_{d} \sin \phi\right)}{\sin ^{2} \phi} \\
\frac{d C_{P}}{d x}=\frac{2 \cdot \lambda_{r} \cdot \sigma F(1-a)^{2}\left(C_{l} \sin \phi-C_{d} \cos \phi\right)}{\sin ^{2} \phi}
\end{gathered}
$$

where $x=r / R$.

At high axial induction factors, the momentum theory represented by equations 3.12 and 3.13 break down, as it indicates reversed flow in the wake. Subsequently, the force coefficients predicted are inaccurate due to the misrepresentation of the flow. The Bhul correction factor is implemented to correct for high axial induction factors, as used in [103, 42] and presented below.

$$
a=\frac{18 F-20-3 \sqrt{C_{n}(50-36 F)+12 F(3 F-4)}}{36 F-50}
$$

where $C_{n}$ represents the normal force coefficient, and $\mathrm{F}$ represents the tip loss factor, as will be presented in Section 3.2.1.

\subsubsection{Tip loss correction}

\subsubsection{Prandtl's tip loss correction model}

Prandtl first introduced a tip loss correction by simplifying the vortex sheet shed by the blades to a series of vortex planes moving together with the wake [28]. Glauert [30] further modified the correction to take the form presented in equation 3.21 . 


$$
F=\frac{2}{\pi} \cos ^{-1}\left[\exp \left(-\frac{B(R-r)}{2 r \sin \varphi}\right)\right]
$$

where $\varphi$ is the local relative velocity angle.

\subsubsection{Goldstein's tip loss correction model}

Goldstein [29] solved for the exact solution of the flow around marine propellers using the vortex method. However, due to the complexity during implementation, Goldstein's equations are more commonly represented using charts. Subsequently, equations have been fitted to the data detailed in the charts, as shown in equation 3.22, and found in Batten et al. [4].

$$
\begin{aligned}
F & =\frac{2}{\pi} \cos ^{-1}\left(\frac{\cosh (x f)}{\cosh (f)}\right) \\
f & =\frac{B}{2 x \tan \varphi}-\frac{1}{2}
\end{aligned}
$$

where $x=r / R$. This set of equations is known as the Goldstein momentum averaging factor. Prandtl's tip loss function and the Goldstein's momentum averaging factor are designed to correct the induced velocities in the BEM equations.

It is noted that Prandtl and Goldstein tip loss correction factors were developed by studying lightly loaded rotors [31], and hence might not be as accurate when the rotor was more heavily loaded. Shortcomings of Prandtl tip loss function have also been found by mathematical analysis [39] and through comparison with CFD $[36,37]$. Attempts have been made to improve the models, or come up with better models. However, some of them have been found to be mathematically inconsistent [31,32], or not easily implemented in the current BEM framework due to the need to access databases [38]. As such, the Prandtl tip loss function in the form of equation 
3.21 is used.

\subsubsection{Shen's tip correction model}

Noting the inconsistencies in Wilson and Lissaman [31], De Vries [32], Shen et al. [33] introduced a correction based on Prandtl tip loss function to the force coefficients used in the BEM equations, instead of correcting the induced velocity. This is to account for the fact that although the forces on a blade tip are supposed to decrease to zero with pressure equalisation, BEM however often produce a non-zero force at the tip. The corrections work in the manner as shown in eq. 3.23.

$$
\begin{aligned}
C_{n}^{r} & =F_{1} C_{n} \\
C_{t}^{r} & =F_{1} C_{t}
\end{aligned}
$$

where $C_{n}$ and $C_{t}$ are the normal and tangential force coefficients respectively. $F_{1}$ is proposed to take the following form as expressed in equation 3.24, similar to equation 3.21 .

$$
F_{1}=\frac{2}{\pi} \cos ^{-1}\left[\exp \left(-g \frac{B(R-r)}{2 r \sin \varphi}\right)\right]
$$

The final form of $g$ is shown in eq. 3.25, after the function is fitted using experimental data.

$$
g=\exp [-0.125(B \lambda-21)]+0.1
$$

where $\lambda$ represents the global Tip Speed Ratio (TSR). 


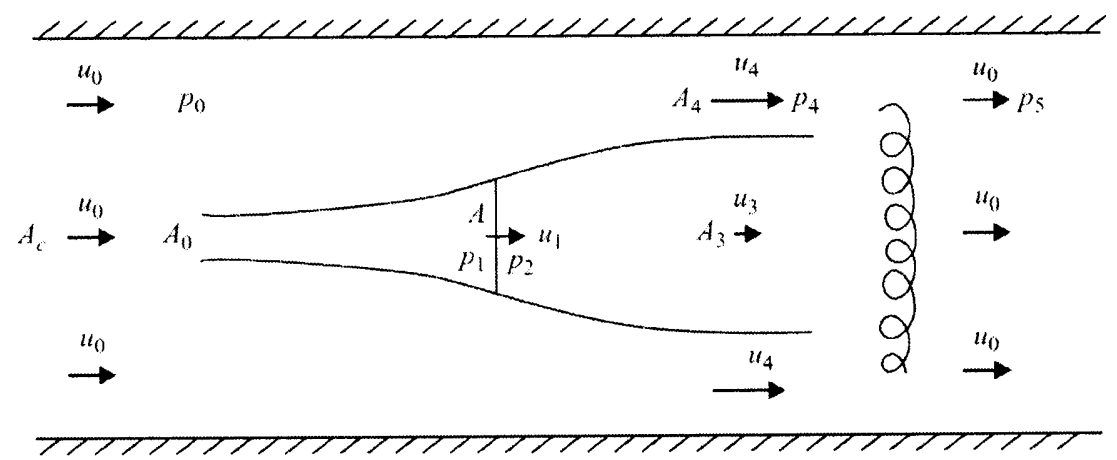

Figure 3.3: Illustration of a turbine being placed in a bounded flow [5]

\subsection{Blockage correction models}

The blockage correction models presented in this section can be derived by considering the actuator disk model presented in section 3.1 placed in a bounded flow, as illustrated in figure 3.3. In addition to the assumption of the LMADT that flow rotation is ignored, uniform flow is also assumed in the different segments of flows.

A brief outline of the blockage correction models to be used is presented in this section. More details of the models can be found in their respective references.

The first model is derived by Garrett and Cummins [5] using the principles of continuity and conservation of momentum in a channel. The result is a correction factor that is only dependent on the blockage ratio of the channel, as illustrated in the equations below.

$$
\begin{gathered}
C_{T, B}=\frac{C_{T}(1+B)}{(1-B)^{2}} \\
C_{P, B}=\frac{C_{P}}{(1-B)^{2}}
\end{gathered}
$$




$$
B=\frac{A}{A_{C}}
$$

where $C_{T, B}$ and $C_{P, B}$ represents the coefficients of thrust and power under blocked conditions respectively, and $B$ represents the blockage ratio of the channel.

The second model is proposed by Whelan et al. [76], based on the work by Maskell [75]. It says that the increased force experienced by an object in a blocked flow is as if the object is exposed to the accelerated flow created by the blockage. Whelan et al. [76] presented a model that includes the effect of free-surface adjustments due to changes in pressure in the fluid. However, the case for solid walls are presented below.

$$
\begin{gathered}
\tau=\frac{u_{4}}{u_{0}}=\frac{1-\alpha+\sqrt{B-2 \alpha B+\alpha^{2}\left(1-B+B^{2}\right)}}{1-B} \\
C_{T}=\tau^{2}-\alpha^{2} \\
\alpha=\frac{u_{3}}{u_{0}} \\
\frac{C_{T, B}}{\tau^{2}}=C_{T}
\end{gathered}
$$

It is seen that the second blockage correction also takes into account the thrust coefficient of the turbine, in addition to the blockage ratio. Incidentally, equation 3.29 is also found in the works of Garrett and Cummins $[5]$.

The third model is a modified form of Glauert's correction developed by Barnsley and Wellicome [73] and used by Bahaj et al. [1]. The equations are obtained as a result of continuity and momentum balance in the far-field 
before and after the turbine plane.

$$
\begin{gathered}
\frac{u_{1}}{u_{3}}=\frac{-1+\sqrt{1+B\left(\left(u_{4} / u_{3}\right)^{2}-1\right)}}{B\left(u_{4} / u_{3}-1\right)} \\
\frac{u_{0}}{u_{3}}=\frac{u_{4}}{u_{3}}-B \frac{u_{1}}{u_{3}}\left[\frac{u_{4}}{u_{3}}-1\right] \\
\frac{u_{0}}{u_{3}}=1 / \sqrt{\frac{C_{T}}{\left[\left(u_{4} / u_{3}\right)^{2}-1\right]}}
\end{gathered}
$$

Equations 3.34 and 3.35 are solved iteratively until a value of $u_{0} / u_{3}$ is found to satisfy both equations.

\subsection{Concluding remarks}

Models presented in this chapter are often seen in isolation. Part of the objective of this thesis is to determine the accuracy of the different models by performing comparisons between the different models. In addition, it is noticed that many of the models assume an inviscid flow. Although this is done to keep the models simple, it is not representative of real flows. As such, differences arising from this difference will also be studied and presented in Chapter 6. 


\section{Chapter 4}

\section{CFD model}

This chapter will describe the Computational Fluid Dynamics model that will be used and expanded on in the subsequent chapters. The model takes reference from an actuator disk experiment conducted by Myers et al. Validation is performed for the CFD model against experimental results, and possible explanations are given when discrepancies exists. The actuator disk is used to represent the presence of the tidal turbine, and the porous zone and momentum sink method are investigated for the implementation of the actuator disk. In addition, a new approach to interpreting the results obtained from simulations is introduced. 


\subsection{Methodology}

The thrust equation is conventionally written as in equation 4.1 and obtained by re-arranging equation 3.10 in Chapter 3 .

$$
T=\frac{1}{2} \rho u_{0}^{2} A C_{T, 0}
$$

where $u_{0}$ represents the undisturbed free-stream velocity, $A$ represents the turbine swept area and $C_{T, 0}$ represents the turbine's thrust coefficient. $u_{0}$ is taken to be the velocity in the channel at the turbine's location in its absence.

However, although $u_{0}$ can be measured, it might not be the velocity that is experienced by the turbine. The two quantities that can be measured from the numerical model are $T$ and $u_{T}$, which are the thrust force and the velocity through the turbine. In this present study, equation 4.1 is re-expressed as equation 4.2 by utilising the relations established in the actuator disk theory presented in Section 3.1. This is similar to the approaches used by other authors in representing their turbines in numerical models [104, 105].

$$
T=\frac{1}{2} \rho u_{T}^{2} A\left(\frac{4 a}{1-a}\right)
$$

Subsequently, $u_{E}$ and $C_{T, E}$, the velocity that is experienced by the turbine and the thrust coefficient experienced respectively, can be calculated using equations 4.3 and 4.4 after determining the axial induction factor, $a$, from equation 4.2 .

$$
\begin{gathered}
u_{E}=\frac{u_{T}}{1-a} \\
C_{T, E}=4 a(1-a)
\end{gathered}
$$


The thrust equation is thus expressed as in equation 4.5.

$$
T=\frac{1}{2} \rho u_{E}^{2} A C_{T, E}
$$

The approach taken in using equations 4.2-4.4 eliminates the need for any assumptions in determining the reference values, and allows for the direct calculation of all required variables based on quantities that can be measured from the model. However, this method might be difficult to implement on a physical system as it is not easy to measure the velocity through the device.

The velocity ratio, $\gamma$, is then calculated from the CFD results using equation 4.6, and is used as a measure of the effect of blockage on the performance of a turbine. The coefficient of thrust as defined by equation 4.1 can then be obtained from equation 4.7 .

$$
\begin{gathered}
\gamma=\frac{u_{E}}{u_{0}} \\
C_{T, B}=\gamma^{2} C_{T, E}
\end{gathered}
$$

\subsection{Numerical Model}

A numerical model of an actuator disk experiment performed in a circulating water channel [97] was made. This results in a computational domain with a width of $1.35 \mathrm{~m}$, and a height of $0.3 \mathrm{~m}$. The domain extends 20 and 40 turbine diameters before and after the first turbine respectively. For computational efficiency, half the domain was considered.

Velocity and turbulence inlet profiles were used, which were obtained using a pre-run of the simulation utilising a uniform velocity and turbulence inlet profile. The profiles were extracted at the point where they matched the experimental data [7] at 5 turbine diameters before the first turbine position. 
Table 4.1: Grid convergence index of the three levels of mesh created

\begin{tabular}{c|cccccccc} 
& & \multicolumn{8}{|c}{ Normalised centreline velocity } \\
& Mesh size & $-5 \mathrm{D}$ & $-1 \mathrm{D}$ & $4 \mathrm{D}$ & $7 \mathrm{D}$ & $11 \mathrm{D}$ & $15 \mathrm{D}$ & $20 \mathrm{D}$ \\
\hline Coarse & 957705 & 0.984 & 0.994 & 1.004 & 1.01 & 1.018 & 1.024 & 1.032 \\
Medium & 2113383 & 0.982 & 0.992 & 1.002 & 1.008 & 1.015 & 1.022 & 1.029 \\
Fine & 4475936 & 0.981 & 0.991 & 1.001 & 1.007 & 1.013 & 1.02 & 1.027 \\
GCI (\%) & & 0.44 & 0.57 & 0.65 & 0.77 & 0.86 & 0.95 & 1.07
\end{tabular}

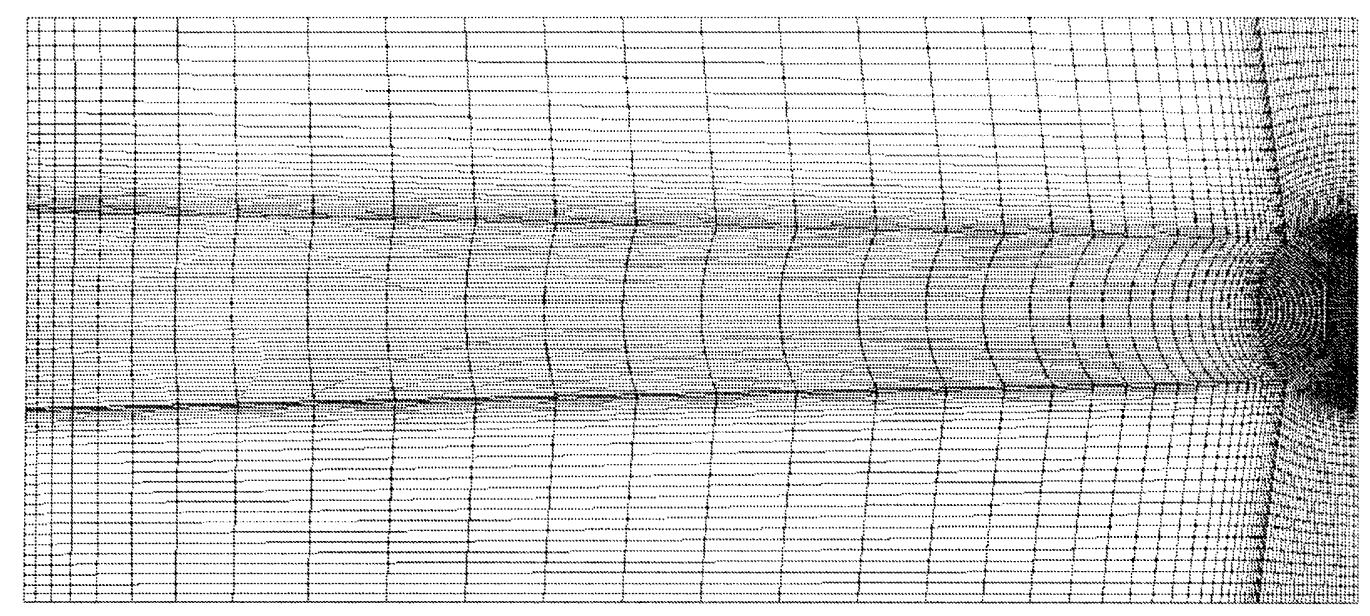

Figure 4.1: Cross section of the medium mesh

The non-slip wall condition was assigned to both the bed and the side wall, while the free-slip wall condition was assigned to the water surface. The standard wall function is used for the non-slip walls, where the law-of-thewall is used to calculate the flow quantities in the first cell away from the wall [106]. The outlet was assigned the pressure outlet condition.

A structured grid was generated using ICEM CFD. Three levels of meshes were created, with the grid convergence index (GCI) [107] at several points in the domain being presented in Table 4.1. It is noticed that there is little difference in the quantities measured using the medium and fine mesh. Thus, the medium mesh is used. A cross section of the medium mesh is presented in Figure 4.1. 
2nd order discretisation was applied to the flow and turbulent quantities, while discretisation for pressure was set to be standard.

The commercial finite volume solver ANSYS FLUENT 13.0 is used to solve the model.

\subsubsection{Turbulence representation}

Turbulence in the flow is characterised by the fluctuation of the flow velocity. Using Reynolds decomposition, the flow velocity, $u$, can be seperated into two components as seen in equation 4.8.

$$
u=\bar{u}+u^{\prime}
$$

where $\bar{u}$ is the mean flow velocity, and $u^{\prime}$ is the fluctuation of the velocity about the mean.

The amount of turbulence in the flow is quantified using the Turbulence Intensity, $I$, which is defined in equation 4.9 [108].

$$
I \equiv \frac{u_{r m s}^{\prime}}{\bar{u}}
$$

where $u_{r m s}^{\prime}$ is the root mean square of the fluctuation of the flow velocity. The turbulent kinetic energy, $k$, is defined as in equation 4.10.

$$
k=\frac{1}{2}\left(\overline{u_{x}^{\prime 2}}+\overline{u_{y}^{\prime 2}}+\overline{u_{z}^{\prime 2}}\right)
$$

where $u_{x}^{\prime}, u_{y}^{\prime}$ and $u_{z}^{\prime}$ are the instantaneous velocity fluctuations in the different directions. 
For flows where it is assumed that the turbulent fluctuation in the 3 directions are the same, equation 4.10 then becomes equation 4.11 .

$$
k=\frac{3}{2} \overline{u^{\prime 2}}
$$

Thus,

$$
u_{r m s}^{\prime} \equiv \sqrt{\frac{1}{3}\left(\overline{u_{x}^{\prime 2}}+\overline{u_{y}^{\prime 2}}+\overline{u_{z}^{\prime 2}}\right)}=\sqrt{\frac{2}{3} k}
$$

Substituting equation 4.12 into equation 4.9 and rearranging, $k$ can be expressed in terms of $I$ [108].

$$
k=\frac{3}{2} I^{2} \bar{u}^{2}
$$

The turbulent dissipation rate, $\varepsilon$, and the specific dissipation rate, $\omega$, can than be determined using equations 4.14 and 4.15 respectively [108].

$$
\begin{gathered}
\varepsilon=C_{\mu}^{\frac{3}{4}} \frac{k^{\frac{3}{2}}}{l} \\
\omega=\frac{k^{\frac{1}{2}}}{C_{\mu}^{\frac{1}{4}} l}
\end{gathered}
$$

$C_{\mu}$ is a dimensionless constant, and its value is taken to be 0.09 . $l$ represents the turbulent length scale.

\subsubsection{Actuator disk model}

The actuator disk model is chosen to represent the turbine as it is the most similar model to the Lanchester-Betz theory used in the various blockage correction models. 
The actuator disk can be modelled either through the use of a momentum sink or by the use of porous media. Both methods have been used extensively by researchers.

The porous media formulation is presented in equation 4.16 as provided by ANSYS FLUENT [108]. Pressure difference across the surfaces bounding the porous media creates the thrust force on the flow.

$$
\triangle p=\frac{1}{2} \rho C_{2} u_{T}\left|u_{T}\right| \triangle m
$$

$C_{2}$ represents the inertial resistance factor, which is determined with the method outlined in [109] with the thrust coefficient as the input. $u_{T}$ represents the velocity through the porous media and $\Delta m$ represents the thickness of the porous media.

The size of the momentum sink as defined by ANSYS FLUENT [108] is determined by the momentum change that occurs in the volume over which the sink is applied. In this case, this is equivalent to the amount of thrust applied to the flow, and is illustrated in equation 4.17.

$$
S=\frac{T}{V}
$$

$S$ presents the momentum sink, $T$ is the thrust exerted by the turbine on the flow, and $V$ is the volume of fluid over which the momentum sink is applied.

A turbulence source is also added to simulate the turbulence generated by a physical disk. An approximation for the maximum amount of turbulence intensity, $\triangle I_{m}$, added into the wake is presented in [7], and shown in equation 4.18.

$$
\triangle I_{m}=0.362\left[1-\left(1-C_{T}\right)^{\frac{1}{2}}\right]
$$


The corresponding amount of turbulence kinetic energy and dissipation can then be calculated based on equations 4.13-4.15.

As recommended by [7], a length scale of $0.005 \mathrm{~m}$ was used for the turbulence source. Due to the lack of data for other thrust coefficients, the same length scale is used regardless of the thrust coefficient of the actuator disk.

\subsection{Validation}

The numerical model was validated against the experimental results provided in [7], in which an experiment was performed with an actuator disk for a thrust coefficient of 0.86 . The momentum sink approach for representing the actuator disk was first used. Common two-equation turbulence models were tested during the validation process using the numerical model described above, and results for the different turbulence models are shown from figures 4.2-4.5. Velocity, turbulence intensity and pressure plots along the vertical plane through the centre of the turbine for the different cases can be found in Appendix A.

The vertical turbulence intensity profiles in the numerical domain show that the turbulence intensity tend to abruptly decrease at the top of the domain. This is thought to be due to the use of the free-slip condition for the top surface. However, it is not likely to affect the rest of the numerical results.

Figure 4.2 shows that all the turbulence models can reasonably reproduce the vertical velocity and turbulence intensity profile in the empty flume. However, the standard k-epsilon turbulence model is seen to produce the best prediction for the velocity and turbulence intensity profiles when the 


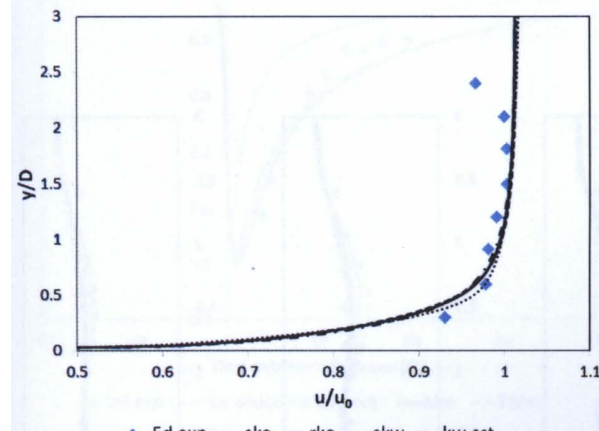

- -5d exp — ske .....rke -..-skw - . kw-sst

(a)

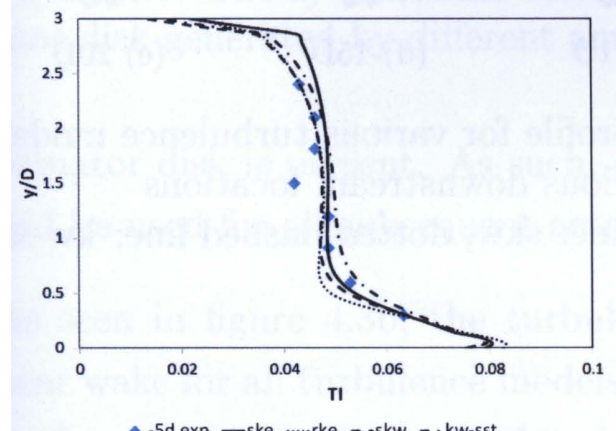

(c)

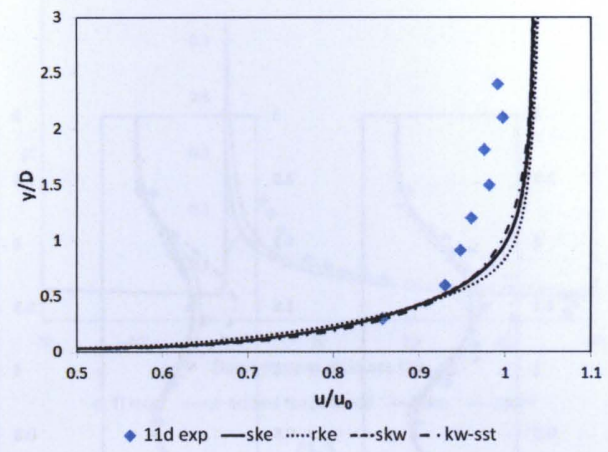

(b)

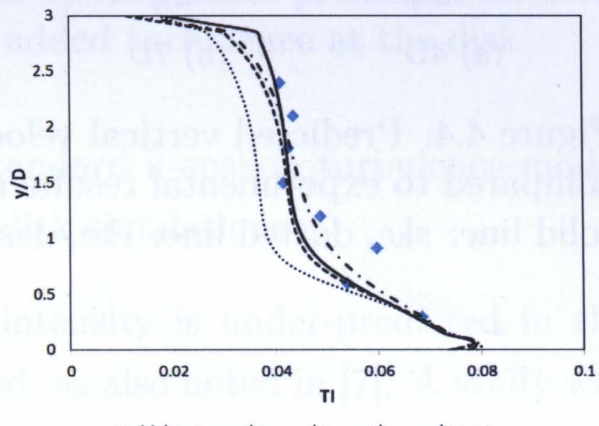

(d)

Figure 4.2: The velocity $(\mathrm{a}, \mathrm{b})$ and turbulence intensity $(\mathrm{c}, \mathrm{d})$ profiles of the flume generated by the different turbulence models.

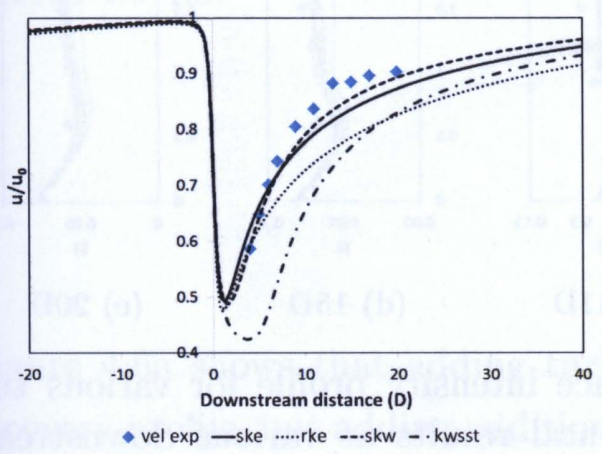

(a)

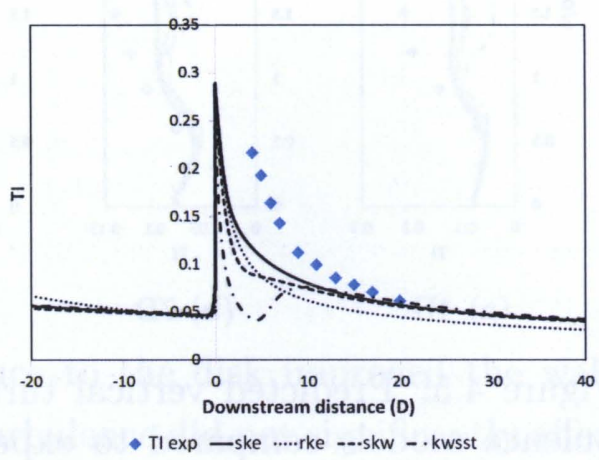

(b)

Figure 4.3: The a) centreline velocity and b) turbulence profiles of an actuator disk generated by the different turbulence models. 


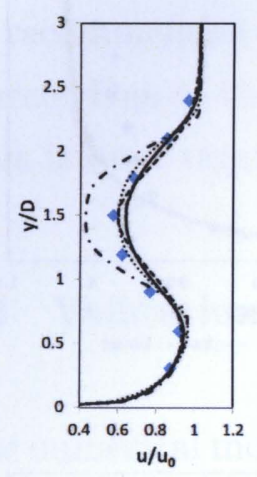

(a) $4 \mathrm{D}$

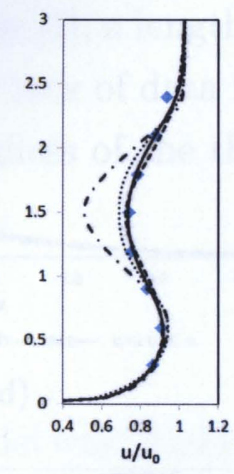

(b) $7 \mathrm{D}$

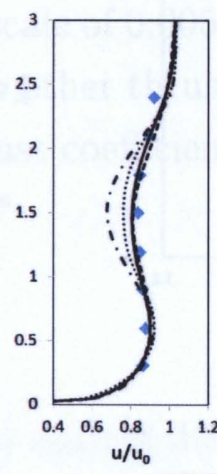

(c) $11 \mathrm{D}$

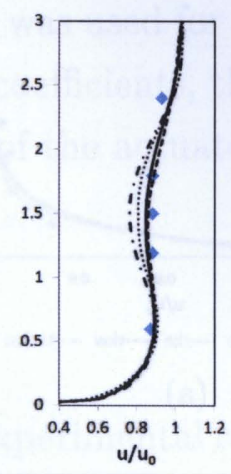

(d) $15 \mathrm{D}$

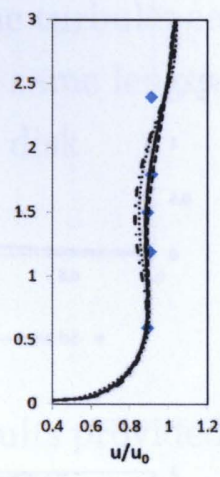

(e) $20 \mathrm{D}$

Figure 4.4: Predicted vertical velocity profile for various turbulence models compared to experimental results at various downstream locations solid line: ske, dotted line: rke, dashed line: skw, dotted-dashed line: kw-sst

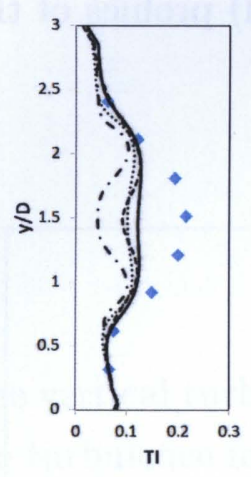

(a) $4 \mathrm{D}$

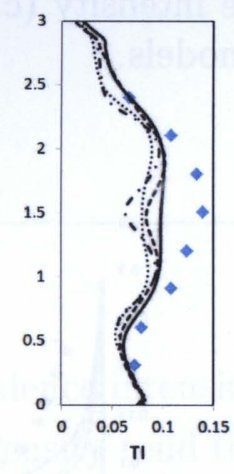

(b) $7 \mathrm{D}$

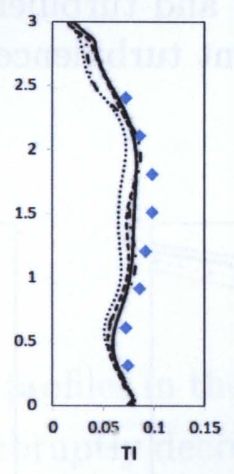

(c) $11 \mathrm{D}$

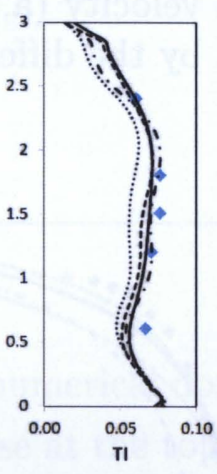

(d) $15 \mathrm{D}$

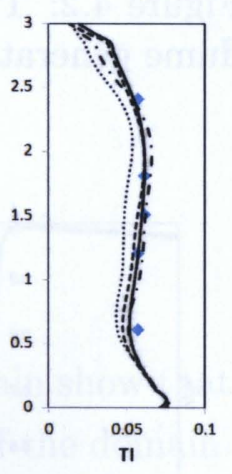

(e) $20 \mathrm{D}$

Figure 4.5: Predicted vertical turbulence intensity profile for various turbulence models compared to experimental results at various downstream locations

solid line: ske, dotted line: rke, dashed line: skw, dotted-dashed line: kw-sst 


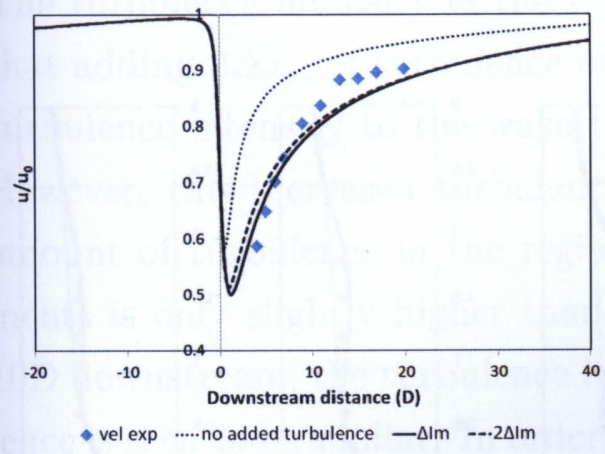

(a)

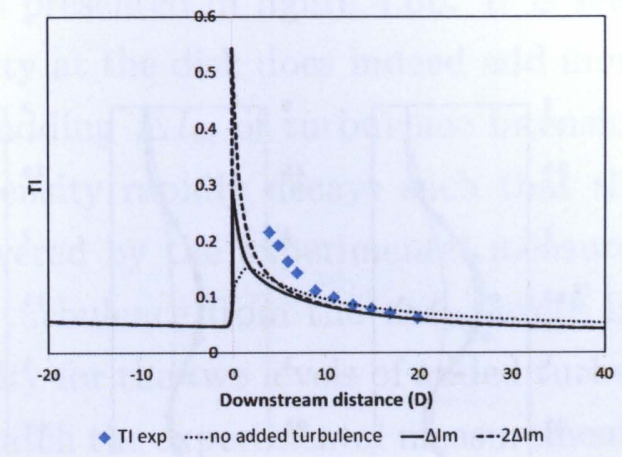

(b)

Figure 4.6: The a) centreline velocity and b) turbulence profiles of an actuator disk generated by different amount added turbulence at the disk

actuator disk is present. As such, the standard k-epsilon turbulence model will be used for all subsequent actuator disk simulations.

As seen in figure $4.3 \mathrm{~b}$, the turbulence intensity is under-predicted in the near wake for all turbulence models tested, as also noted in [7]. A study was performed to investigate whether the amount of turbulence added by the disk affected the wake recovery and the turbulence intensity in the wake. Added turbulence intensity levels of $0, \triangle I_{m}$ and $2 \Delta I_{m}$ were tested, where $\triangle I_{m}$ refers to the amount calculated from equation 4.18. Results for the centreline and for vertical profiles at various locations downstream are presented in figures 4.6-4.8.

Figure 4.6a shows that adding turbulence to the disk improved the wake recovery profile, but adding additional turbulence did not significantly affect the wake recovery. Wake recovery for the $2 \triangle I_{m}$ level was marginally faster within $10 \mathrm{D}$ downstream compared to the $\triangle I_{m}$ level. By 20D, wake recovery for the 2 added turbulence levels were similar. 


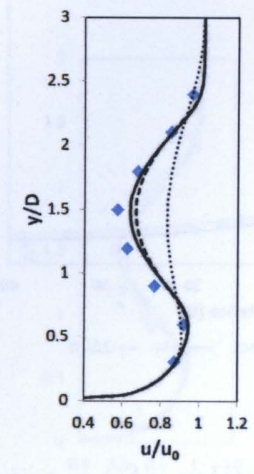

(a) $4 \mathrm{D}$

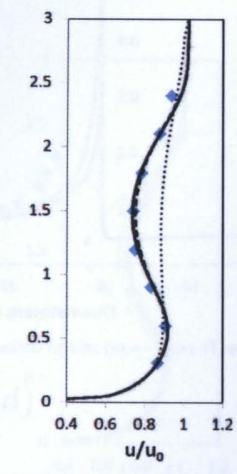

(b) $7 \mathrm{D}$

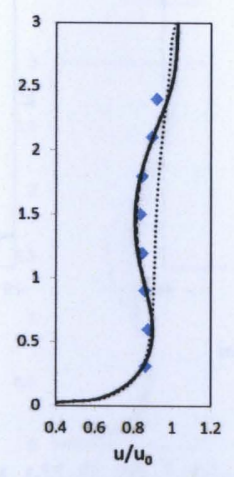

(c) $11 \mathrm{D}$

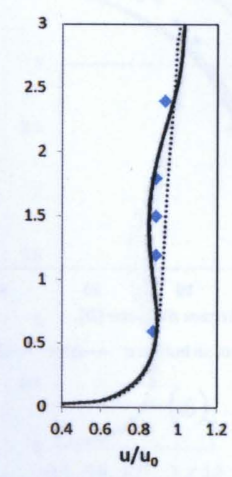

(d) $15 \mathrm{D}$

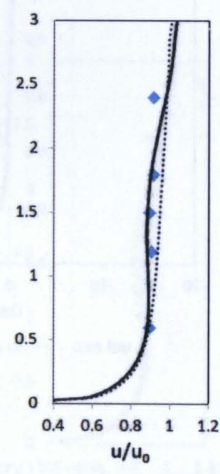

(e) $20 \mathrm{D}$

Figure 4.7: Predicted vertical velocity profile for various amounts of turbulence added at the disk compared to experimental results at various downstream locations

No added turbulence: dotted line, $\triangle I_{m}$ added: solid line, $2 \triangle I_{m}$ added: dashed line

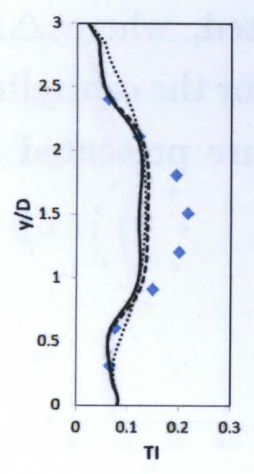

(a) $4 \mathrm{D}$

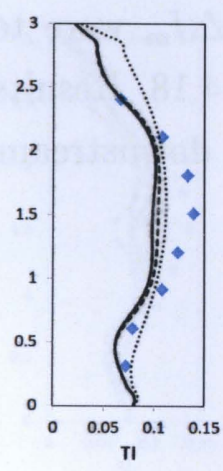

(b) $7 \mathrm{D}$

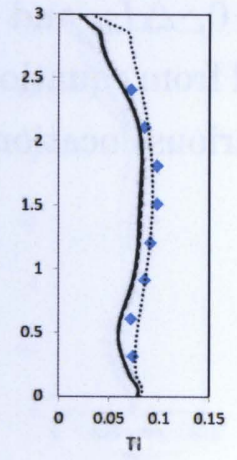

(c) $11 \mathrm{D}$

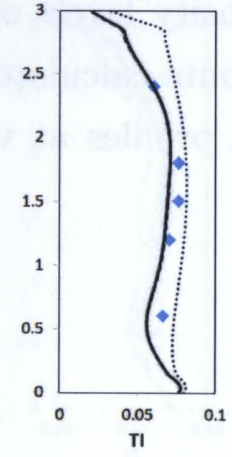

(d) $15 \mathrm{D}$

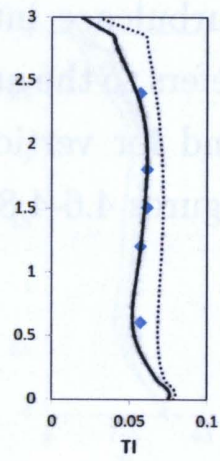

(e) $20 \mathrm{D}$

Figure 4.8: Predicted vertical turbulence intensity profile for various amounts of turbulence added at the disk compared to experimental results at various downstream locations

No added turbulence: dotted line, $\Delta I_{m}$ added: solid line, $2 \Delta I_{m}$ added: dashed line 
The turbulence intensity in the wake is presented in figure $4.6 \mathrm{~b}$. It is seen that adding $2 \triangle I_{m}$ of turbulence intensity at the disk does indeed add more turbulence intensity to the wake than adding $\triangle I_{m}$ of turbulence intensity. However, this increased turbulence intensity rapidly decays such that the amount of turbulence in the region covered by the experimental measurements is only slightly higher than the turbulence from the $\triangle I_{m}$ level. By 10D downstream, the turbulence intensity for the two levels of added turbulence is seen to be similar. In order to match the experimental measurements of the turbulence in the wake, a far larger amount of turbulence intensity is needed to be added at the disk. However, doing so is not physically realistic and would affect the wake recovery profile as well. Hence, the $\triangle I_{m}$ of turbulence intensity added at the disk was chosen for the rest of this thesis for the best balance between having an accurate wake recovery profile and turbulence intensity profile in the wake.

Differences between the numerical and experimental results in the wake recovery and turbulence decay in the wake could be due to the assumption that the turbulence in the wake is isotropic when using the 2-equation turbulence models, while turbulence in the actual flume might not be isotropic. Also, turbulence generated by the physical disk might not be generated at the disk location as currently assumed, but a short distance downstream when the turbulent eddies are formed after the flow passes through the disk.

Wake generated by the momentum sink approach was next compared with that generated by the porous media formulation and presented in figure 4.9. It is seen that the wake generated by both methods are almost identical, with the difference being the predicted velocity through the turbine.

\subsection{Discussion}

Although the two methods of modelling the actuator disk serve to provide a resistance to the flow, they do so by different means. The momentum 


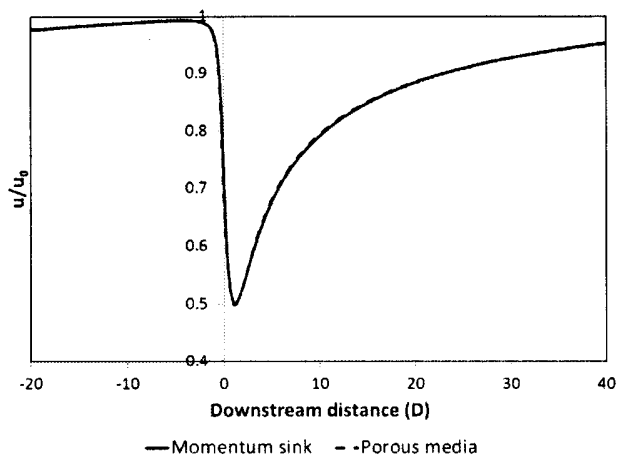

Figure 4.9: Comparison of the wake generated by the momentum sink approach and the porous media formulation

sink approach is useful when the thrust on the flow is known, but the thrust coefficient of the device is unknown. An example of this situation is when thrust readings are recorded from experiments and the thrust coefficient is to be determined. On the other hand, the porous media formulation is useful when the thrust on the flow is unknown, but the thrust coefficient of the device is known.

The two methods can be utilised to predict the performance of a device outside its experimental environment. The momentum sink approach can first be used to determine the free-stream equivalent thrust coefficient of a device after measuring its thrust coefficient under experimental blocked conditions. Using the free-stream equivalent thrust coefficients, the porous media formulation can then be used to measure the thrust exerted by that device in other blocked conditions. 


\section{Chapter 5}

\section{Effects of Reynolds number and tip loss models on the accuracy of $\mathrm{BEMT}^{1}$}

This chapter studies the effect of various aspects of the BEMT model on the predicted power and thrust coefficient of a turbine. The tip loss models as introduced by Prandtl, Goldstein and Shen et al. were compared against experimental results of 2 turbines found in literature. In addition, the effects of varying Reynolds number over the blades on the predicted power and thrust at various operating conditions are studied. The Prandtl tip loss model was found to provide the best match with experimental results. Allowing the lift and drag coefficients of the blade to vary with Reynolds number resulted in a higher power coefficient being predicted at higher rotation speeds.

\footnotetext{
${ }^{1}$ This section is published substantially as: Koh, W.X.M., Ng, E.Y.K.. Effects of Reynolds number and different tip loss models on the accuracy of BEM applied to tidal turbines as compared to experiments. Ocean Engineering 2016;111:104-115
} 


\subsection{Introduction}

Chapter 2 discusses various factors that affect the accuracy of the BEMT. Among them, the choice of tip loss model has a significant impact on the accuracy of the predicted power and thrust coefficients. Although there are many tip loss models available, no studies comparing the accuracy of the different models available in literature exists to the author's knowledge.

Accuracy of the airfoil data that is an input to the model is also often overlooked. Throughout the complete turbine operating range from the cutin to cut-out velocity, the turbine blade experiences a wide range of Reynolds numbers. There is also a considerable variation of Reynolds numbers over the blade span. It is known that the lift and drag of $2 \mathrm{D}$ airfoil sections are dependent on the Reynolds number of the airfoil. Hence, for the same airfoil section at the same position along the blade, its lift and drag coefficients would change throughout the full operating range of the turbine. However, existing BEMT studies $[4,35,110]$ often display only 1 set of lift and drag coefficients for their airfoils. Conditions at which the lift and drag coefficients are obtained are also often not mentioned. Mason-Jones [110] included the BEMT code used in his thesis, and his expression for the coefficient of lift is seen to be constant for all the blade sections.

Thus, this chapter aims to compare the effect of the different tip loss models, as well as the effect of Reynolds number on the accuracy of the predicted power and thrust coefficients.

\subsection{Rotor models}

Due to extensive literature available, the rotor presented by Bahaj et al. [1] is used, with the blade sectional data as given in Table 5.1. The rotor was previously used in a series of cavitation tunnel and tow tank experiments measuring the power and thrust under different operating conditions. The rotor is 3 bladed and has a diameter of $800 \mathrm{~mm}$, with the NACA $63-8 \mathrm{xx}$ 
airfoil being used as the cross sections for the blades. The rotor is designed to operate with a hub pitch angle of 20 degrees.

In order to ensure that the results obtained from the rotor by Bahaj et al. [1] is not unique to that rotor alone, the rotor presented by Mycek et al. [2] is also chosen to verify the results by Bahaj et al's rotor. The rotor is used in experiments to study the effect of turbulence on rotor performance. The rotor is also 3 bladed, but with a diameter of $700 \mathrm{~mm}$. The NACA $63-4 \mathrm{xx}$ airfoil was used for the cross sections of the blades, with the blade sectional data included in Table 5.2. Results presented in later sections were obtained at the blade setting in Table 5.2.

\subsection{Methodology}

The BEMT codes presented in section 3.2 were used in this chapter. The Goldstein, Prandtl and Shen tip loss corrections presented in section 3.2.1 were used and compared. The Goldstein and Prandtl tip loss corrections work by modifying the induction factors in the BEMT equations as seen in equations 3.12 and 3.13, whereas Shen correction modifies the force coefficients of the turbine. Thus, its usage can be combined with the other two tip loss corrections.

2D airfoil lift and drag coefficients were obtained by XFOIL. XFOIL is a 2D linear-vorticity panel code coupled with a boundary layer formulation, developed to evaluate low Reynolds number airfoils [111]. This allows the code to take into account viscous effects when computing the lift and drag forces, and to model small to moderate levels of flow separation. Turbulence transition is governed by the $e^{n}$ transition criterion, where $\mathrm{n}$ (Ncrit) is the critical number used to determine transition. A range of Ncrit has been suggested for aerodynamic applications [112], but none has been found for hydrodynamic applications. Results for a series of hydrofoil tests can be found in Molland et al. [6], and they were used to determine the Ncrit that provide the best fit to experimental data. By trial and error, the Ncrit 


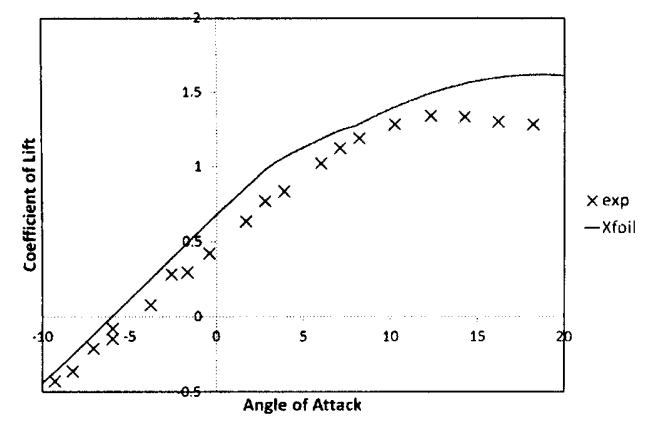

(a)

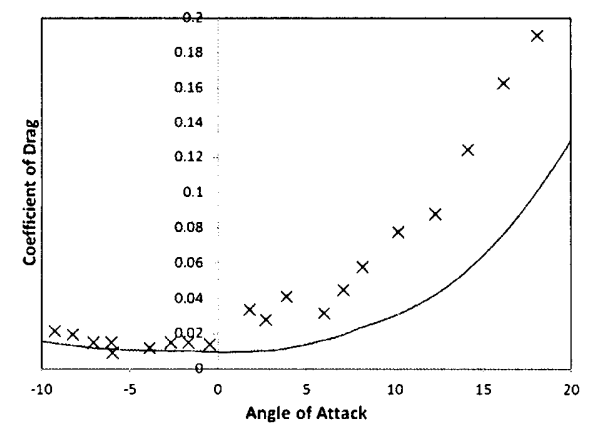

(b)

Figure 5.1: Comparison of the a) lift and b) drag coefficients predicted by XFoil and experiments for the NACA 63-815 airfoil [6]

value of 0.5 was found to provide the best match between the predicted and experimental results, and will be used for subsequent airfoil lift and drag predictions. Figure 5.1 shows the predictions of lift and drag obtained by XFOIL with Ncrit of 0.5 compared to experimental results obtained at a Reynolds number of 800,000 for the NACA $63-815$ airfoil.

It is observed that although lift is slightly over-predicted in the pre-stall region, there is generally good agreement with experimental results. Drag is generally under-predicted, but correlates well to the experimental results for negative angle of attacks (AOA). Angle of attack refers to the angle at which the incoming fluid meets the chord line of an airfoil, which is represented by the angle $\alpha$ in figure 3.2. At positive AOAs, the under-prediction increases with increasing angle of attacks. This suggests that XFOIL might not be able to model separated flows well. As no further experimental data exist for this particular class of airfoils, airfoil data being presented subsequently is assumed to have the same level of accuracy as that presented in Figure 5.1 .

For the turbine presented by Bahaj et al. [1], 2D airfoil data for the airfoils NACA $63-812,63-815,63-818,63-821$ and $63-824$ were obtained by XFOIL for a range of Reynolds number from $0.7 \times 10^{5}$ to $5 \times 10^{5}$, while airfoil data 
for the turbine by Mycek et al. [2] were also obtained for the same range of Reynolds number. The blade chord is used as the reference length for the calculation of the Reynolds number. Figures 5.2 and 5.3 show the the lift and drag coefficient versus angle of attack over the stated range of Reynolds number for NACA 63-815 and NACA 63-418 respectively, while figure 5.4 shows the variation of the lift to drag $(\mathrm{L} / \mathrm{D})$ ratio versus angle of attack for the stated range of Reynolds number for the two airfoils.

It can be seen that the airfoil lift and drag vary over the selected range of Reynolds number. Generally, airfoil lift coefficient increases with increasing Reynolds number, while the drag coefficient decreases. In addition, the $\mathrm{L} / \mathrm{D}$ ratio for both the airfoils generally increase with increasing Reynolds number, and the angle of attack where the maximum L/D ratio happens tend to decrease with increasing Reynolds number.

In wind tunnel tests of low Reynolds number airfoil, it is observed that laminar separation bubbles are observed on the airfoil [113, 114]. They are formed when a laminar flow encounters a strong enough adverse pressure gradient that causes it to separate. Depending on the flow conditions, the separated flow might transit to a turbulent flow and reattach, forming a bubble [115]. The presence of this bubble causes an increase in drag and a decrease in lift. However, this is not observed at the low Reynolds number range of the airfoils presented. This could be due to the fact that the low Ncrit causes the flow to transition early. It is however unclear whether laminar separation bubbles are to be expected for the airfoils being used at the range of Reynolds number stated.

The 3D airfoil correction by Snel et al. [20] is applied to the airfoil data up to the stall delay angle, and the post stall model of Viterna and Corrigan [116] is used to obtain the post stall data.

The usage of airfoil data over a range of Reynolds number is compared to the usage of a single set of airfoil data obtained at a reference Reynolds number. The reference Reynolds number for the turbine is defined to be the average Reynolds number across the blade span-wise at the peak power condition. 
Table 5.1: Blade sectional data for the turbine used by Bahaj et al. [1]

\begin{tabular}{|c|c|c|c|c|}
\hline$r / R$ & $r(\mathrm{~mm})$ & $c / R$ & Pitch distribution $\left({ }^{\circ}\right)$ & $t / c(\%)$ \\
\hline 0.2 & 80 & 0.1250 & 15.0 & 24.0 \\
\hline 0.25 & 100 & 0.1203 & 12.1 & 22.5 \\
\hline 0.3 & 120 & 0.1156 & 9.5 & 20.7 \\
\hline 0.35 & 140 & 0.1109 & 7.6 & 19.5 \\
\hline 0.4 & 160 & 0.1063 & 6.1 & 18.7 \\
\hline 0.45 & 180 & 0.1016 & 4.9 & 18.1 \\
\hline 0.5 & 200 & 0.0969 & 3.9 & 17.6 \\
\hline 0.55 & 220 & 0.0922 & 3.1 & 17.1 \\
\hline 0.6 & 240 & 0.0875 & 2.4 & 16.6 \\
\hline 0.65 & 260 & 0.0828 & 1.9 & 16.1 \\
\hline 0.7 & 280 & 0.0781 & 1.5 & 15.6 \\
\hline 0.75 & 300 & 0.0734 & 1.2 & 15.1 \\
\hline 0.8 & 320 & 0.0688 & 0.9 & 14.6 \\
\hline 0.85 & 340 & 0.0641 & 0.6 & 14.1 \\
\hline 0.9 & 360 & 0.0594 & 0.4 & 13.6 \\
\hline 0.95 & 380 & 0.0547 & 0.2 & 13.1 \\
\hline 1.0 & 400 & 0.0500 & 0.0 & 12.6 \\
\hline
\end{tabular}
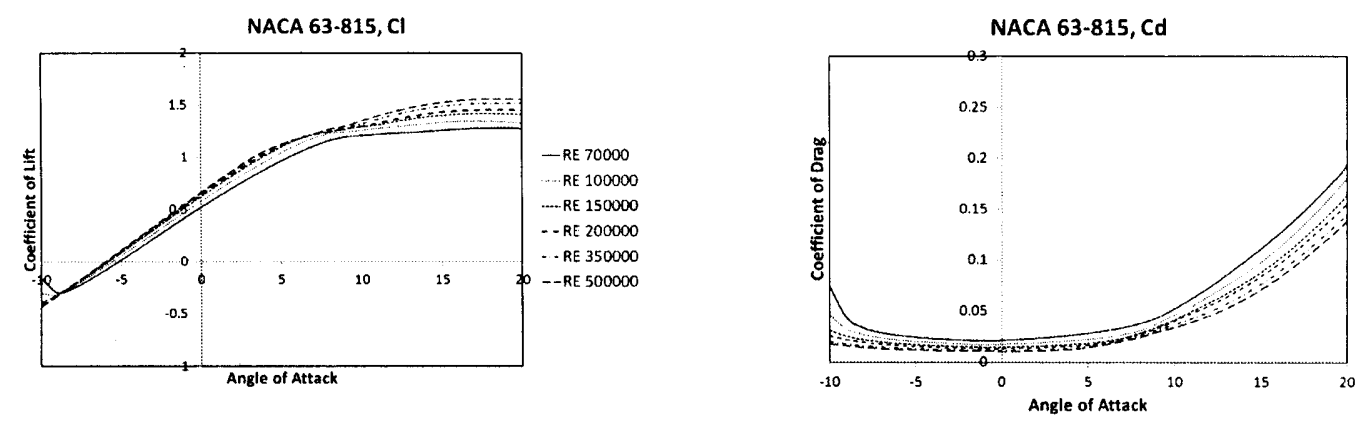

Figure 5.2: Variation of lift and drag coefficients with Reynolds number as predicted by XFOIL for NACA 63-815 used in the turbine by Bahaj et al. [1] 
Table 5.2: Blade sectional data for the turbine used by Mycek et al. [2]

\begin{tabular}{|c|c|c|c|}
\hline$r / R$ & $c / R$ & Pitch distribution $\left({ }^{\circ}\right)$ & $t / c(\%)$ \\
\hline 0.2417 & 0.2474 & 22.1491 & 21.3 \\
\hline 0.285 & 0.2375 & 19.3031 & 21.4 \\
\hline 0.3283 & 0.2259 & 16.9737 & 21.7 \\
\hline 0.3717 & 0.2141 & 15.0538 & 22.0 \\
\hline 0.4150 & 0.2029 & 13.4572 & 22.2 \\
\hline 0.4583 & 0.1925 & 12.1169 & 22.4 \\
\hline 0.5017 & 0.1829 & 10.9815 & 22.5 \\
\hline 0.545 & 0.1743 & 10.0114 & 22.5 \\
\hline 0.5883 & 0.1665 & 9.1761 & 22.4 \\
\hline 0.6317 & 0.1594 & 8.4516 & 22.2 \\
\hline 0.675 & 0.1529 & 7.8191 & 21.9 \\
\hline 0.7183 & 0.1471 & 7.2638 & 21.5 \\
\hline 0.7617 & 0.1418 & 6.7735 & 20.9 \\
\hline 0.8050 & 0.137 & 6.3387 & 20.2 \\
\hline 0.8483 & 0.1325 & 5.9514 & 19.5 \\
\hline 0.8917 & 0.1285 & 5.605 & 18.6 \\
\hline 0.935 & 0.1247 & 5.2941 & 18.0 \\
\hline 0.9783 & 0.1213 & 5.0143 & 18.0 \\
\hline 1 & 0.0655 & 4.8743 & 25.0 \\
\hline
\end{tabular}
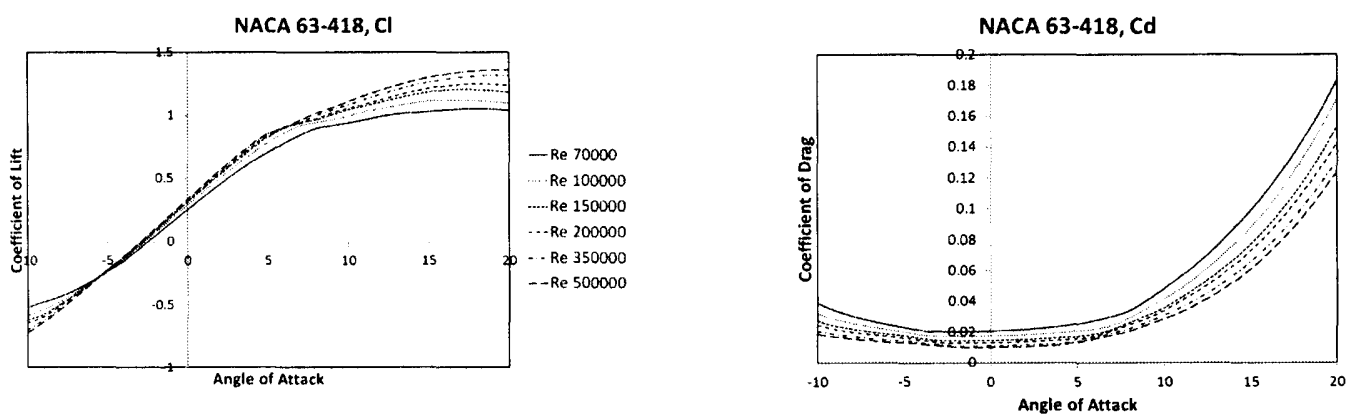

Figure 5.3: Variation of lift and drag coefficients with Reynolds number for NACA 63-418 used in the turbine by Mycek et al. [2] 


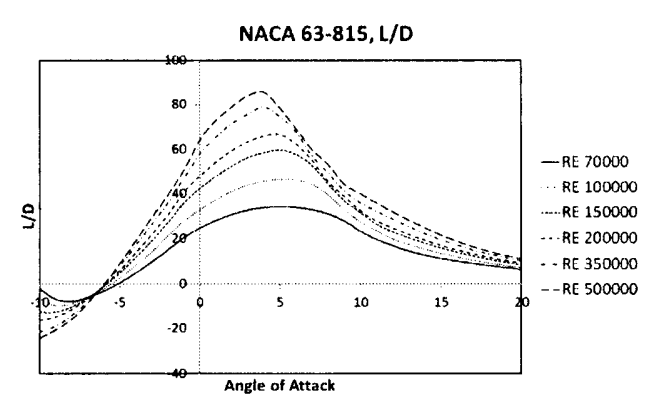

(a)

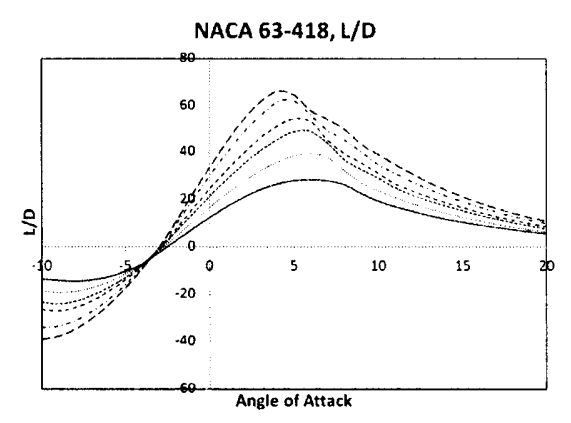

(b)

Figure 5.4: Variation of $\mathrm{L} / \mathrm{D}$ ratio with Reynolds number for a) NACA 63-815 and b) NACA 63-418

For the turbine by Bahaj et al. [1], the reference Reynolds number is $2 \times 10^{5}$ obtained at a TSR of 6 , while it is $1.8 \times 10^{5}$ as obtained at a TSR of 5 for the turbine by Mycek et al. [2].

The results of the tip loss functions are also compared against the experimental results obtained for the rotor used by Bahaj et al. [1] for the blade pitch angles of 20, 25 and 27 degrees, as well as that obtained by the rotor used by Mycek et al. [2].

\subsection{Results and Discussion}

\subsubsection{Effect of Reynolds number variation}

Figures 5.5 and 5.6 compare the effect of using airfoil data obtained with the local sectional Reynolds number as compared with using a single reference Reynolds number for the turbine by Bahaj et al. [1] across 3 different pitch angles for Prandtl and Goldstein tip loss functions. At the design pitch angle of 20 degrees, it is observed that at higher TSRs, the power coefficient predicted with the local sectional Reynolds number is higher that that predicted using a single reference Reynolds number. The thrust coefficient predicted using the local sectional Reynolds number reduces across the whole 
range of TSRs. For pitch angles of 25 and 27 degrees, it can be seen that the power and thrust coefficients predicted with the local sectional Reynolds number are less than that as predicted using the reference Reynolds number. Possible reasons for this can be explained with Figure 5.7.

Figure 5.7 shows the variation of Reynolds number across the operating range of the turbine for the different pitch angles. For the pitch angle of 20 degrees, it is seen that at higher TSRs, the Reynolds number of the blades are higher than that of the reference Reynolds number. This means that the actual lift and drag coefficients experienced by the blade sections at the higher TSRs are respectively higher and lower than that predicted using the reference Reynolds number.

It can be seen that as the blade pitch angle increases and the flow velocity decreases, a larger portion of the turbine is operating below the reference Reynolds number. For the turbine pitch angle of 27 degree, the Reynolds number experienced by the turbine across the whole operating range is less than the reference Reynolds number. As a result, the lift coefficients of the blade sections are reduced and drag coefficient increased as compared to using the reference Reynolds number. This has the effect of reducing the predicted power and thrust coefficients.

Figure 5.8 examines the effect of using the local Reynolds number on the axial and tangential induction factors. It can be seen that most of the changes happen near the tip, with the the axial induction factor being decreased, while the tangential induction factor being increased slightly. This can be explained by examining the changes in the AOA experienced by the blade as a result of using the local Reynolds number, as shown in Figure 5.9.

Figure 5.9 shows an increase in the AOA at the tip as a result of using the local Reynolds number compared to the single reference Reynolds number, resulting in a force vector that is pitched further from the axial direction and closer towards the tangential direction. Subsequently, the axial force coefficient is reduced at the tip while the tangential force coefficient is increased, leading to decreased out of plane bending moments, but increased 


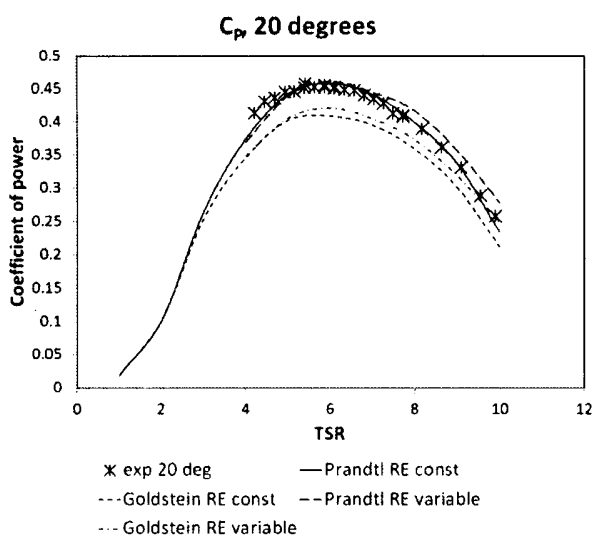

(a) $\mathrm{Cp}, 20$ degrees

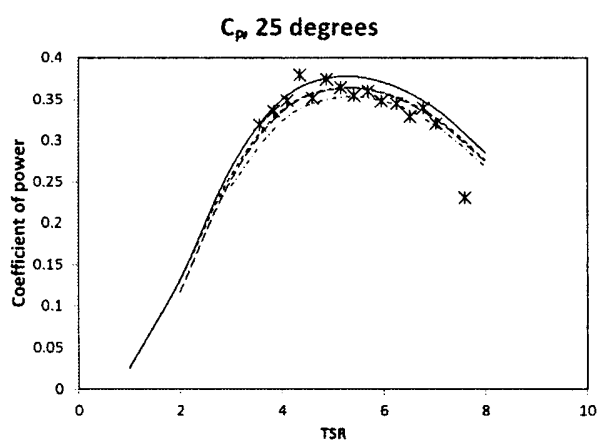

(b) $\mathrm{Cp}, 25$ degrees

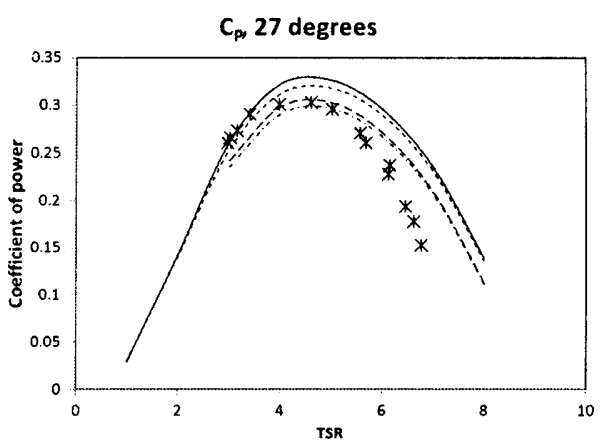

(c) $\mathrm{Cp}, 27$ degrees

Figure 5.5: Effect of using the local Reynolds number on the predicted power coefficient of the turbine by Bahaj et al. [1] for turbine pitch angles of 20, 25 and 27 degrees respectively

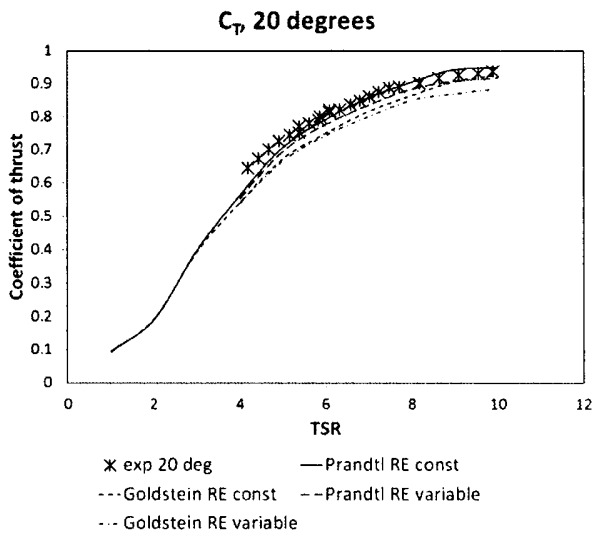

(a) $\mathrm{Ct}, 20$ degrees

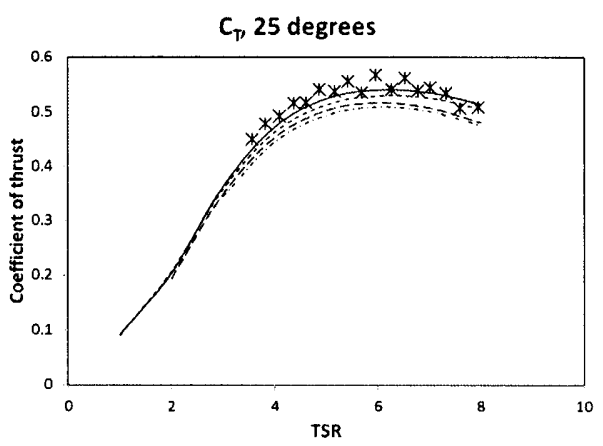

(b) $\mathrm{Ct}, 25$ degrees

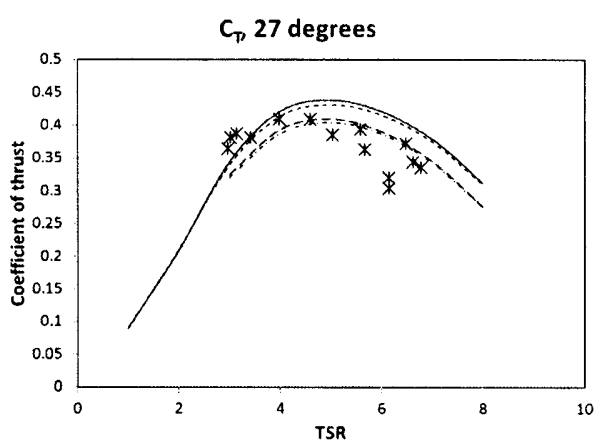

(c) Ct, 27 degrees

Figure 5.6: Effect of using the local Reynolds number on the predicted thrust coefficient of the turbine by Bahaj et al. [1] for turbine pitch angles of 20, 25 and 27 degrees respectively 


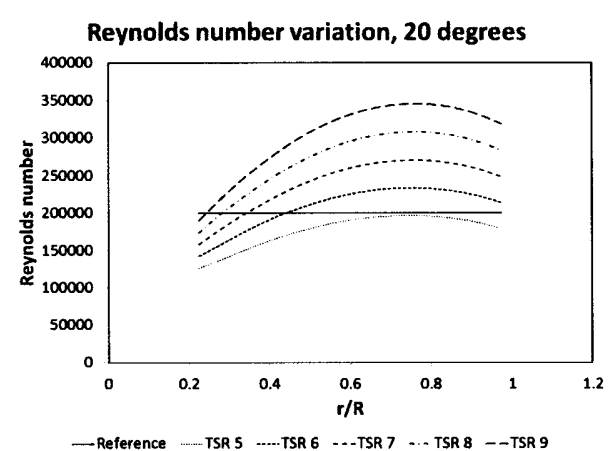

(a) Reynolds number variation, 20 degrees

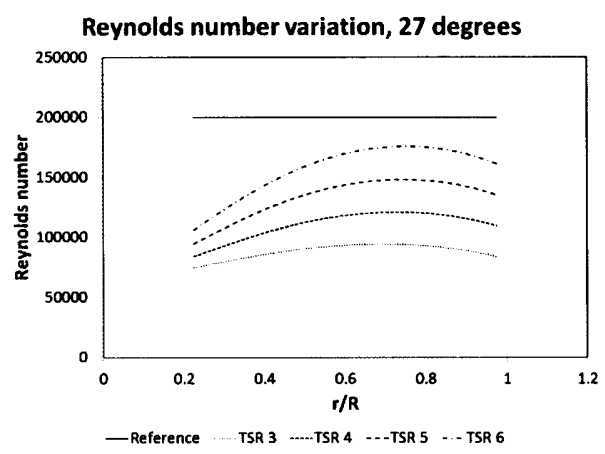

(c) Reynolds number variation, 27 degrees

Figure 5.7: Variation of Reynolds number experienced by the turbine blade used by Bahaj et al. [1] across the whole operational range of TSRs for turbine pitch angles of 20,25 and 27 degrees respectively
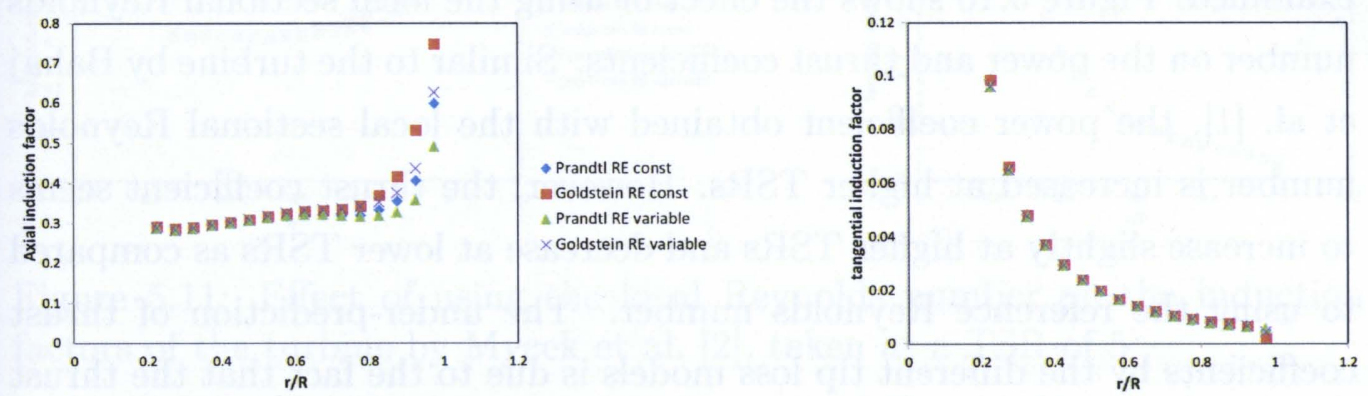

Figure 5.8: Effect of using the local Reynolds number on the induction factors of the turbine by Bahaj et al. [1], taken at a pitch angle of 20 degrees with a TSR of 6 


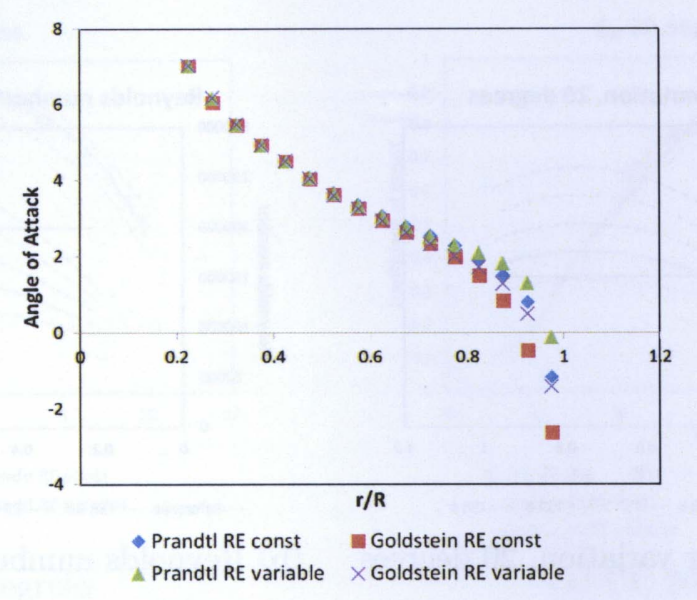

Figure 5.9: Effect of using the local Reynolds number on the angle of attack on the blade of the turbine by Bahaj et al. [1], taken at a pitch angle of 20 degrees with a TSR of 6

edge-wise bending moments.

Examining Figure 5.9, it is noted that the AOA are in the positive pre-stall region throughout most of the blade, which means that the drag experienced by the blade is under-estimated. This could imply that the power coefficient is overestimated and the thrust coefficient underestimated. On the other hand, it might also suggest that the actual AOA on the blade is different from what is predicted.

For further verification purposes, the turbine of Mycek et al. [2] was also examined. Figure 5.10 shows the effect of using the local sectional Reynolds number on the power and thrust coefficients. Similar to the turbine by Bahaj et al. [1], the power coefficient obtained with the local sectional Reynolds number is increased at higher TSRs. However, the thrust coefficient seems to increase slightly at higher TSRs and decrease at lower TSRs as compared to using the reference Reynolds number. The under-prediction of thrust coefficients by the different tip loss models is due to the fact that the thrust coefficient provided by Mycek et al. [2] is for the whole structure, while that predicted by BEMT is produced only by the blades.

Figure 5.11 shows the effect of using the local sectional Reynolds number on 

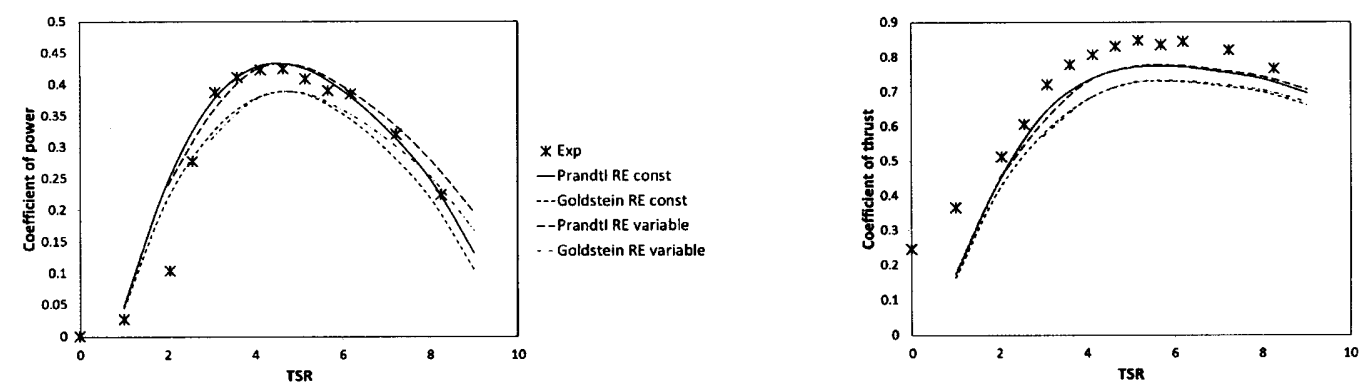

Figure 5.10: Effect of using the local Reynolds number on the predicted power and thrust coefficient of the turbine by Mycek et al. [2]
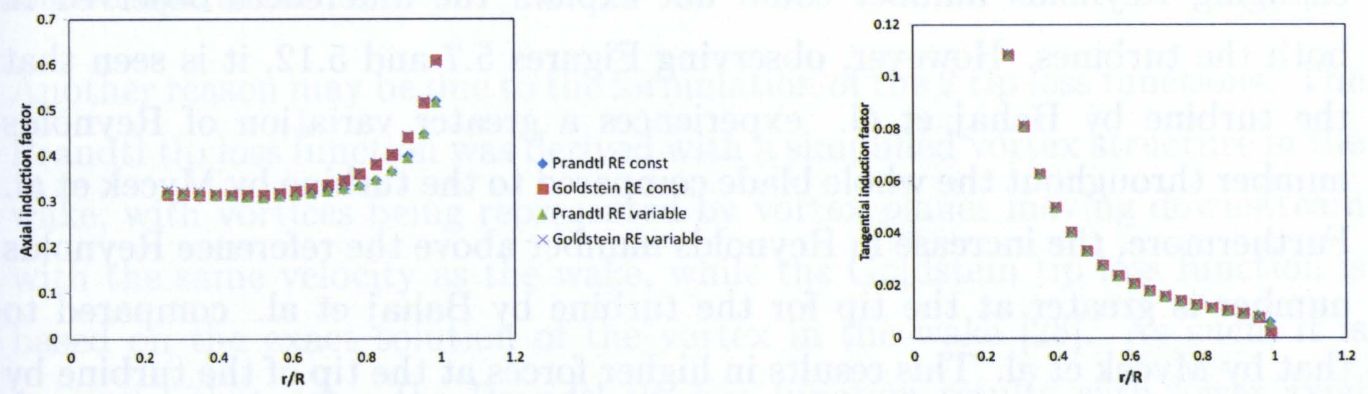

Figure 5.11: Effect of using the local Reynolds number on the induction factors of the turbine by Mycek et al. [2], taken at a TSR of 5 


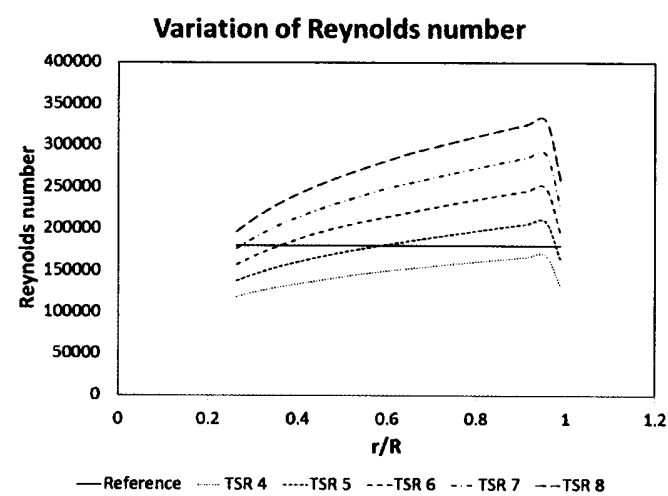

Figure 5.12: Variation of Reynolds number for the turbine by Mycek et al. [2] for various TSRs

the induction factors for the turbine by Mycek et al. It is noticed that there are no significant changes in both the axial and tangential induction factor as a result of using the local Reynolds number. This is in contrast to Figure 5.8 , where there are noticeable changes in the induction factors at the blade tip when the local sectional Reynolds number is used.

Examining Figures 5.2 and 5.3, it is seen that the lift and drag coefficients of both families of airfoils vary in the same manner with regards to increasing Reynolds number. Thus, differences in airfoil performance with respect to changing Reynolds number could not explain the differences observed in both the turbines. However, observing Figures 5.7 and 5.12, it is seen that the turbine by Bahaj et el. experiences a greater variation of Reynolds number throughout the whole blade compared to the turbine by Mycek et al. Furthermore, the increase in Reynolds number above the reference Reynolds number is greater at the tip for the turbine by Bahaj et al. compared to that by Mycek et al. This results in higher forces at the tip of the turbine by Bahaj et al. compared to that predicted using a single reference Reynolds number, leading to greater changes compared to the turbine by Mycek et al.

Figures 5.8 and 5.11 both show that the axial induction factor is more than 0.5 at the tip, which indicates that there is flow reversal in the wake if the actuator disk theory is to be enforced. However, this cannot happen 
in reality and turbulent mixing of the wake with the bypass flow happens instead [11].

\subsubsection{Effect of tip loss functions}

For the study on tip loss functions, results obtained using the local sectional Reynolds number is used, as it is physically more accurate. Only the results for the turbine by Bahaj et al. are shown in this section, as the behaviour for the tip loss functions is expected to be similar across different turbines.

From Figures 5.5 and 5.6, it is clear that the Prandtl tip loss function generally provides a higher prediction for both the coefficients of power and thrust over the turbine operating range for all the 3 blade pitch angles, and subsequently better accuracy. Also, it can be seen that the difference between the tip loss functions decreases as the blade pitch angle increases. A check with the axial induction factor showed that the rotor is most heavily loaded at a blade pitch angle of 20 degree, and the loading subsequently decreased for blade pitch angles of 25 and 27 degrees. This may be a reason for the wide differences in performance between Prandtl and Goldstein tip loss function at a blade pitch angle of 20 degree, as they are not derived for heavily loaded operations.

Another reason may be due to the formulation of the 2 tip loss functions. The Prandtl tip loss function was derived with a simplified vortex structure in the wake, with vortices being represented by vortex planes moving downstream with the same velocity as the wake, while the Goldstein tip loss function is based on the exact solution of the vortex in the wake [28]. As such, it is proposed that using the Prandtl tip loss function results with lower axial induction factors at the tip in order to ensure uniform velocity in the wake as much as possible, so as to prevent the vortex planes from being distorted. This is seen in Figures 5.8 and 5.11. A lower axial induction factor is the result of larger AOA, which results in a larger tangential force component, and subsequently higher power production being predicted. 
Figures 5.13 and 5.14 introduce the effect of Shen's correction to Prandtl and Goldstein's tip loss functions. Shen's correction tends to increase the prediction of power coefficient at higher TSR, and decreases the thrust coefficient for the whole operational range of TSR, which is similar to the effects of using the local sectional Reynolds number instead of a single reference Reynolds number as illustrated in Figures 5.5 and 5.6. This can be explained by examining the effects of Shen's correction on the induction factors as predicted by Prandtl and Goldstein tip loss functions.

Figure 5.15 presents the effect of Shen's correction on the predicted induction factors. It is clear that Shen's correction acts to decrease the axial induction factor at the tip, as is also obtained by Shen et al. [33]. Figure 5.15 further shows that the rotational induction factor remains virtually unchanged by Shen's correction. A smaller axial induction with an unchanged rotational induction factor would mean an increase in the AOA of the blade section, as seen in Figure 5.16. However, as the relative inflow angle has changed together with the AOA, it is then unclear how the loading at the tip is changed.

Figures 5.17 and 5.18 show the effect of Shen's correction on the axial and tangential force coefficients. As can be seen in Figure 5.17, the normal force coefficient at the tip has indeed been decreased, in line with the rationale for Shen's correction. Although it is not obvious in Figure 5.18, the tangential force coefficient has been increased slightly by Shen's correction. This increment is also observed to increase with higher TSR. This has the effect of increasing the power coefficient, reducing the thrust coefficient at the blade tip, and subsequently for the whole turbine.

Examining Figures 5.13 and 5.14, the effect of Shen's correction has seemed to have reduced the accuracy of the tip loss models used in this study. However, it is unclear which of the predicted blade loading profile is more accurate without more information. Furthermore, there are inaccuracies in the airfoil lift and drag data which introduce further uncertainties. Obtaining more accurate lift and drag is unlikely to affect the general behaviours of 


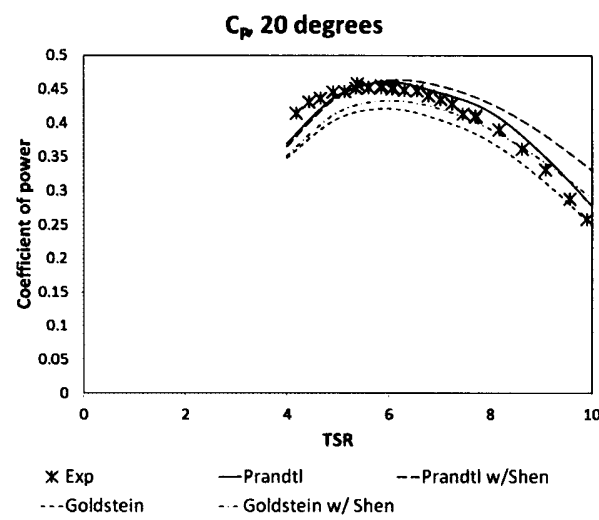

(a) $\mathrm{Cp}, 20$ degrees

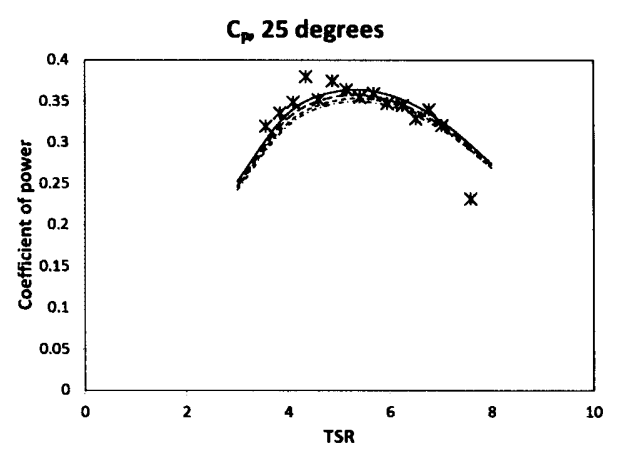

(b) $\mathrm{Cp}, 25$ degrees

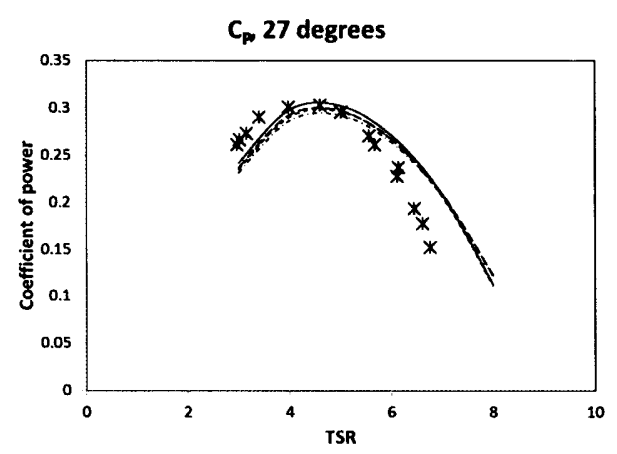

(c) $\mathrm{Cp}, 27$ degrees

Figure 5.13: Effect of Shen's correction on the predicted power coefficient of the turbine by Bahaj et al. [1] for turbine pitch angles of 20,25 and 27 degrees respectively
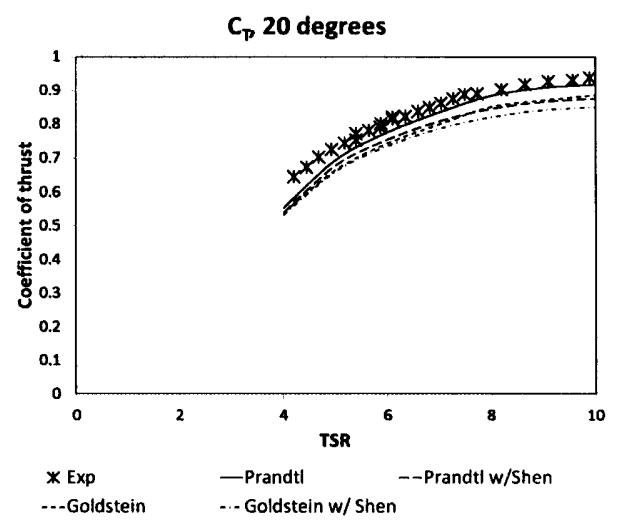

(a) Ct, 20 degrees

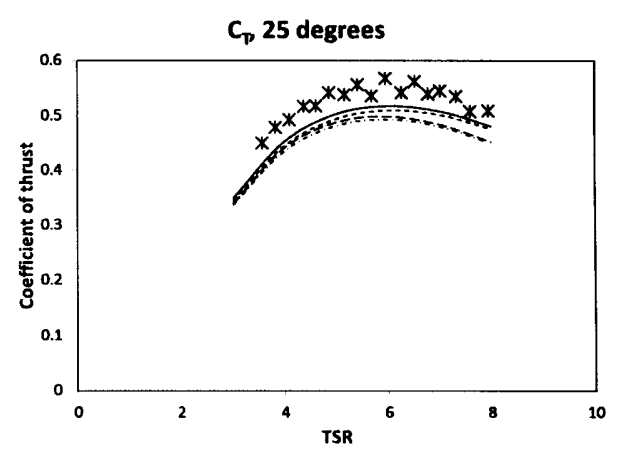

(b) Ct, 25 degrees

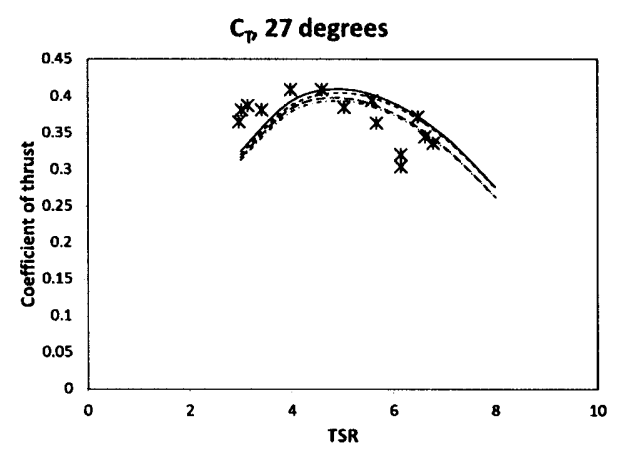

(c) Ct, 27 degrees

Figure 5.14: Effect of Shen's correction on the predicted thrust coefficient of the turbine by Bahaj et al. [1] for turbine pitch angles of 20,25 and 27 degrees respectively 

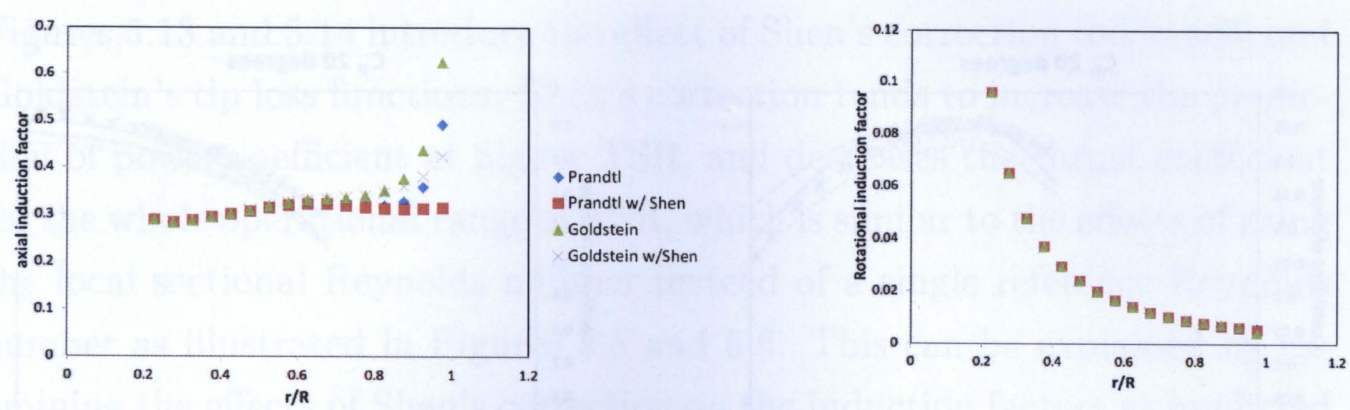

Figure 5.15: Effect of Shen's correction on the induction factors of the turbine by Bahaj et al. [1] for a TSR of 6 and blade pitch angle of 20 degrees

the tip loss models, although the relative accuracies of the different models is likely to be affected.

\subsection{Concluding remarks}

A study was done on the effect of using airfoil data obtained with the local sectional Reynolds number versus that achieved with a single reference Reynolds number. At the design pitch angle, using airfoil data obtained from the local sectional Reynolds number yielded a higher power coefficient at higher TSRs for the 2 turbines tested, while the effect on the thrust coefficient was different for each turbine.

The effect of different types of tip loss functions was also studied. The Prandtl tip loss function consistently predicted higher power and thrust coefficients than Goldstein tip loss function, with the differences increasing with increasing axial induction factors. Shen's correction served to increase the predicted power coefficient of the turbine at higher TSR, similar to the effect of using the local sectional Reynolds number as compared to a single reference Reynolds number, while the thrust coefficient was reduced for the whole operational range of TSR.

Uncertainties in the settings used in XFoil for hydrodynamic applications are also expressed, and this has the ability to affect the accuracy of the results. 


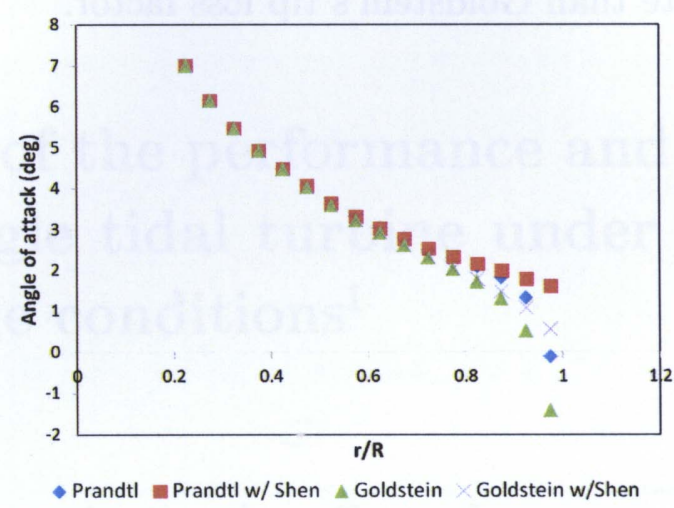

Figure 5.16: Effect of Shen's correction on the angle of attack for a TSR of 6 and blade pitch angle of 20 degrees

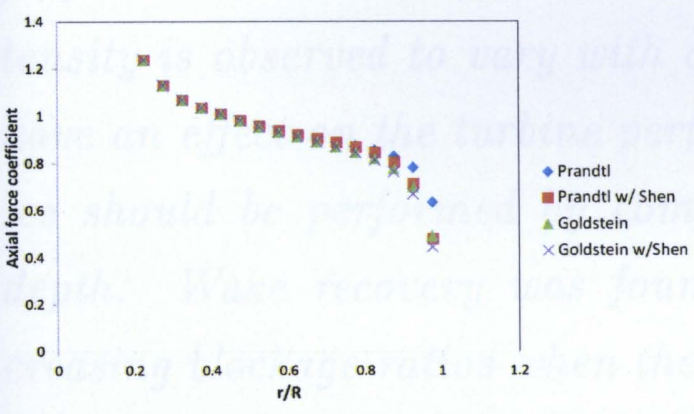

Figure 5.17: Effect of Shen's correction on the axial force coefficient at a TSR of 6 for a blade pitch angle of 20 degrees

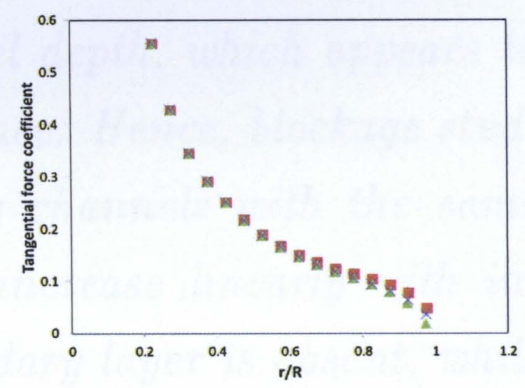

Figure 5.18: Effect of Shen's correction on the tangential force coefficient at a TSR of 6 for a blade pitch angle of 20 degrees 
Overall, considering the inaccuracies in the airfoil lift and drag data and limited validation data available, the Prandtl tip loss factor has been found to be more accurate than Goldstein's tip loss factor. 


\section{Chapter 6}

\section{CFD study of the performance and wake recov- ery of a single tidal turbine under various flow and blockage conditions ${ }^{1}$}

This chapter investigates the effect of various channel and flow parameters on the performance and wake recovery of a tidal turbine through the use of CFD. Modelling the boundary layer caused a reduction in the predicted thrust produced by the turbine as compared to the boundary layer not being modelled. Also, the presence of the boundary layer results in an aspect ratio where the turbine performance is maximised. The rate of decay of turbulence intensity is observed to vary with channel depth, which appears to have an effect on the turbine performance. Hence, blockage studies should be performed by comparing channels with the same depth. Wake recovery was found to increase linearly with increasing blockage ratios when the boundary layer is absent, while the near wake is minimally affected by blockage effects.

\footnotetext{
${ }^{1}$ This section is published substantially as: Koh, W.X.M., Ng, E.Y.K.. A CFD study on the performance of a tidal turbine under various flow and blockage conditions. Renewable Energy 2017, In Press
} 


\subsection{Introduction}

In the operation of a tidal turbine in open waters, it is inevitable that blocked conditions are encountered. Blocked conditions are also experienced in water tunnel experiments. However, it is impossible for water tunnel experiments to simulate every kind of condition than a tidal turbine might face in open water condition.

It is known that a turbine operating in a bounded flow will experience some interference in its behaviour compared to when operating in free-stream conditions [71], where the flow around the turbine and its wake is able to expand freely without any interference. As such, it is of interest to be able to translate data from water tunnel experiments into free-stream equivalent data, and then transform them again into blocked data for use in open water conditions. Majority of the experimental and numerical works involve translating data obtained from water tunnel into free-stream equivalent data. Bahaj et al. [1] first used a modified form of Glauert's correction for propellers to correct their experimental data. This was subsequent picked up and used by other authors [61, 74]. Whelan et al. [76] used a theory proposed by Maskell [75], which was originally used in wind tunnels. The basis for the corrections is that for the device to experience the same thrust in a free-stream environment as that experienced in a tunnel environment, the device will experience an equivalent free-stream velocity, which is different from the tunnel velocity [71].

However, there are few studies on predicting the performance of a turbine in blocked conditions given the free-stream performance data. Garrett and Cummins [5] used the Lanchester-Betz theory to develop a model to predict the thrust and power produced by a turbine in a partially blocked flow. This was expanded by Nishino and Willden [83, 84] to develop a model for an array partially blocking the channel. It is also theoretically possible to use the corrections used by Bahaj et al. and Whelan et al. in reverse to predict the performance of a turbine in blocked flow given its free-stream performance. 
One common thread among the different correction models is that the blockage ratio of the channel is the only variable that is used in determining the performance of a turbine in a channel, whereas others have investigated other factors affecting turbine performance. Keogh et al. [117] conducted flume experiments studying the effect of blockage and different channel aspect ratios on the performance of an actuator disk. Nishino and Willden [48] conducted CFD experiments utilising the actuator disk model to study the effect of channel area and aspect ratio on the thrust and wake recovery of the turbine. Kolekar and Banerjee [68] also did CFD experiments to study the effect of channel area, as well as position of the turbine in the water column on the performance of their turbine. Maganga et al. [94] conducted rotor experiments to study the effect of turbulence intensity of the upstream flow on the turbine's performance, while Mycek et al. [2] also studied the effect of the upstream velocity in addition to turbulence intensity. Both studies reported that turbine performance dropped with increasing turbulence intensity.

Study of the wake recovery is also important as it helps determine the distance between rows in large arrays. While there are more studies on the effect of blockage on the performance of turbines, there are comparatively fewer studies on the effect of blockage on the wake recovery of turbines. This is perhaps due to a lack of test facilities with a working section that is long enough. McTavish et al. [8] studied the effects of blockage on the initial wake expansion of a tidal turbine, and found that the effect of blockage on the initial wake expansion was significant. Giles et al. [118] studied the wake recovery of a turbine in different water depths, and found that an optimal water depth to turbine diameter ratio exists for the fastest wake recovery.

This chapter attempts to study the effect of various flow quantities and geometric relations on the performance and wake recovery of a single turbine by CFD using the actuator disk concept. 
Table 6.1: Cross sectional dimensions and reference velocities of the channels tested

\begin{tabular}{|c|c|c|c|c|}
\hline & Width $(\mathrm{m})$ & Depth $(\mathrm{m})$ & $\begin{array}{c}\text { Reference } \\
\text { velocity }(\mathrm{m} / \mathrm{s})\end{array}$ & Blockage ratio \\
\hline Channel 0 & 1.35 & 0.30 & 0.333 & 0.019 \\
\hline Channel 1 & 4.05 & 0.90 & 0.579 & 0.002 \\
\hline Channel 2 & 0.80 & 0.30 & 0.333 & 0.033 \\
\hline Channel 3 & 0.30 & 0.30 & 0.333 & 0.087 \\
\hline Channel 4 & 0.90 & 0.45 & 0.406 & 0.019 \\
\hline Channel 5 & 0.64 & 0.64 & 0.486 & 0.019 \\
\hline Channel 6 & 0.45 & 0.90 & 0.581 & 0.019 \\
\hline
\end{tabular}

\subsection{Methodology}

The numerical model described in Chapter 4 is expanded to produce 6 other channels with different widths and depth, while maintaining a similar mesh density. The use of the standard k-epsilon turbulence model is maintained. Table 6.1 shows the cross-sectional dimensions of all the channels that are tested, as well as the blockage ratio and the reference velocity for each channel. The reference velocity refers to $u_{0}$ in equation 4.1 , and is such that the Froude number for all channels are similar. The blockage ratio, $B$, is as defined in equation 3.28 .

Channel 0 refers to the channel that is described in Chapter 4. Channel 1 has width and depth 3 times that of channel 0 , and represents the unblocked condition. Channels 2 and 3 maintains the same depth as channel 0 , but has reduced width. Channels 4,5 and 6 has the same cross-sectional area as channel 0 , but with different aspect ratios. Aspect ratio in this study is defined as the ratio of the channel width to the channel depth. The actuator disk is located at mid-depth for all channels.

Actuator disks various thrust coefficients, $C_{T, 0}$, were simulated in the seven channels. The disk has a diameter of $0.1 \mathrm{~m}$ and is placed at mid depth for all channels. The velocity ratio, $\gamma$, defined in equation 4.6 is compared across the different channels and operating conditions. The actuator disk theory 
described in section 3.1 is assumed to be valid for all thrust coefficients and is used for the calculation of $\gamma$.

Boundary conditions were adjusted for the different simulations to test for different parameters, and will be described in Section 6.3.

\subsection{Results and discussion}

\subsubsection{Effect of boundary layer}

Analytical models used for blockage effect predictions are often derived with the implicit assumption that the flow is inviscid due to the use of Bernoulli's equation. However, that assumption is not valid in real world operating conditions as the effects of viscosity are ignored or not taken into account during analysis. One important factor that is often omitted is the effect of the boundary layer on the outcome of predictions. The effect of the boundary layer is critical as current studies in tidal turbine deployment are in relatively shallow waters, meaning that the tidal turbine is encountering part of the boundary layer.

To investigate the effect of boundary layer on the accuracy of predictions, two sets of CFD simulations were performed on the channels described in Section 6.2. The first set of simulations involved setting the bottom and lateral boundaries as non-slip walls and letting the boundary layer develop, while the second set of simulations involved setting all the walls as the symmetry boundary condition. The bottom and lateral non-slip walls for the first set of simulations have the same properties, which results in similar boundary layers being produced in the vertical and lateral direction. Inlet velocity and turbulence profiles for each of the channels were obtained using the same method described in Section 4.2, with the reference velocities being adjusted such that all channels have similar Froude numbers. This is reflected in the column stating the reference velocities for each of the channels in Table 6.1. Velocity and turbulence intensity profiles for all channels simulated can be 


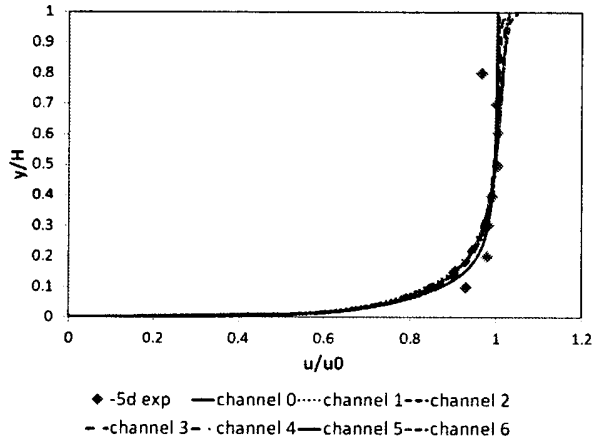

(a)

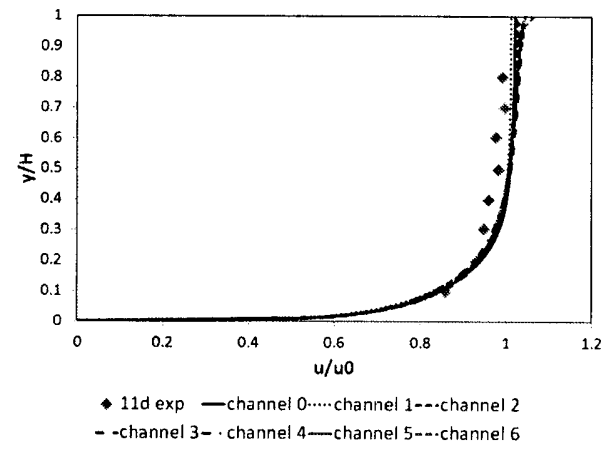

(b)

Figure 6.1: Velocity profiles for all the channels at a) 5 diameters before and b) 11 diameters after the turbine location compared with experimental data [7]

seen in figures 6.1 and 6.2 respectively. Velocities and turbulence quantities at the inlet for the second set of simulations were adjusted such that the velocity and turbulence intensity at the turbine location is similar for both set of simulations. The boundary conditions of the second set of simulations are set to be symmetric, which makes them more similar to the assumptions of the analytical models, and thus taken as the basis for comparisons.

Figure 6.3 shows the relationship between $C_{T, 0}$ which is used as the input for the actuator disk model and $C_{T, E}$ as calculated in equation 4.4 , while figure 6.4 compares the ratio $C_{T, E} / C_{T, 0}$ for all channels. It is seen that $C_{T, E}$ is similar to $C_{T, 0}$ for all the cases tested. Thus, any changes in velocity, $v_{E}$, would correspond to a change in thrust.

Figure 6.5 shows the ratios of velocities for both set of simulations, and figure 6.6 presents the difference between both sets of simulations. Contour 


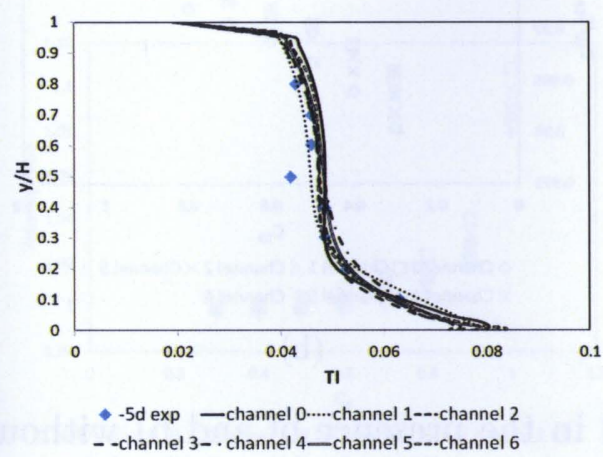

(a)

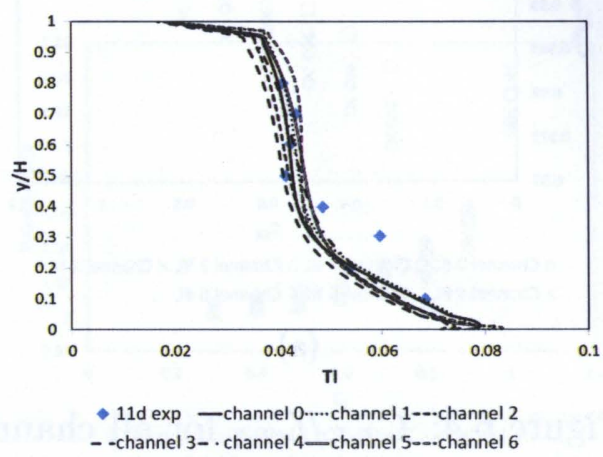

(b)

Figure 6.2: Turbulence Intensity profiles for all the channels at a) 5 diameters before and b) 11 diameters after the turbine location compared with experimental data [7]

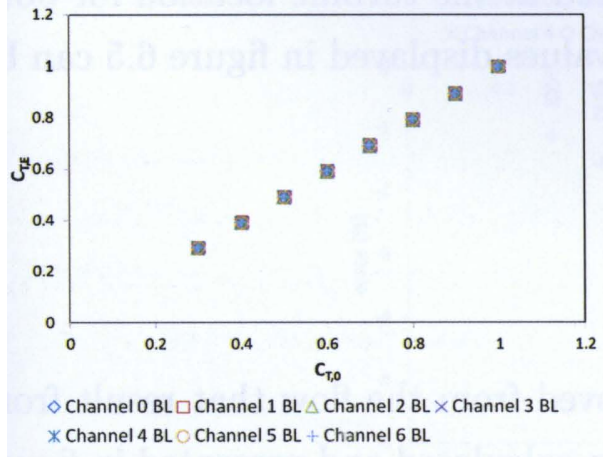

(a)

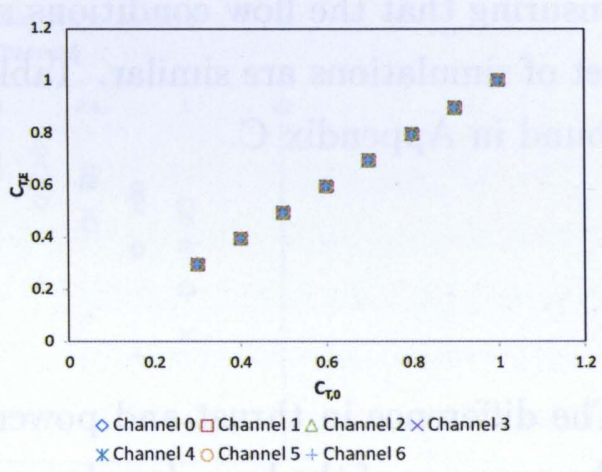

(b)

Figure 6.3: Comparison between $C_{T, 0}$ and $C_{T, E}$ for all channels a) in the presence of and b) without the presence of the boundary layer 


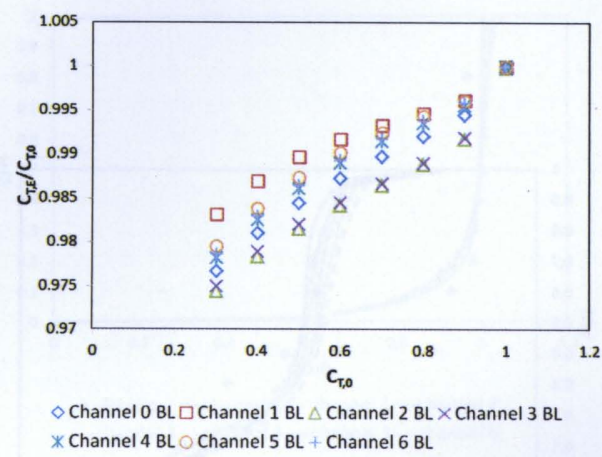

(a)

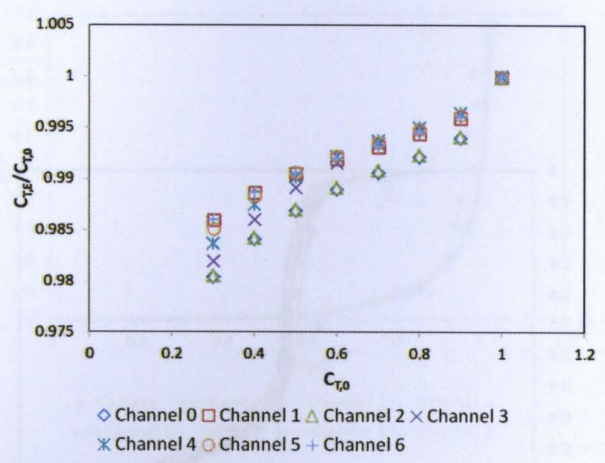

(b)

Figure 6.4: $C_{T, E} / C_{T, 0}$ for all channels a) in the presence of and b) without the presence of the boundary layer

plots of the normalised velocity, turbulence intensity and pressure for the turbine in the different channels operating at a thrust coefficient of 1 can be found in Appendix B. The reference velocities for the different channels shown in table 6.1 were used for the calculation of the normalised velocity and the turbulence intensity for each channel.

It is seen that the velocity ratio measured in the presence of a boundary layer is less than that measured without the boundary layer. This is despite ensuring that the flow conditions measured at the turbine location for both set of simulations are similar. Tables of values displayed in figure 6.5 can be found in Appendix C.

The difference in thrust and power removed from the flow that result from the presence of the boundary layer is then calculated and presented in figure 6.7. The overestimation of thrust when neglecting the presence of the boundary layer might be beneficial to structural designers as it means that there is some measure of safety factor built into the calculations. However, estimation of flow recovery downstream might be affected due to the difference in thrust that is actually experienced. 


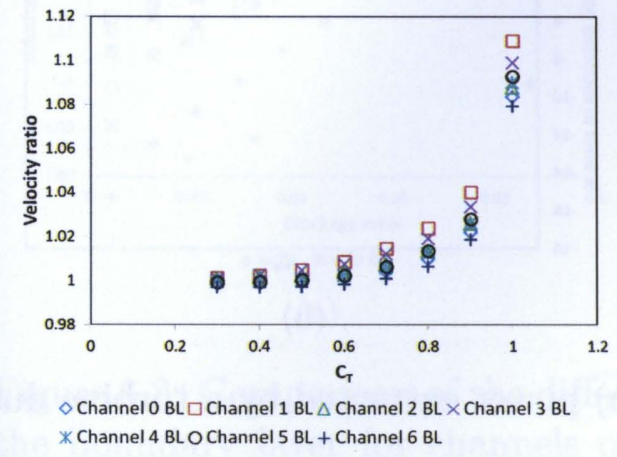

(a)

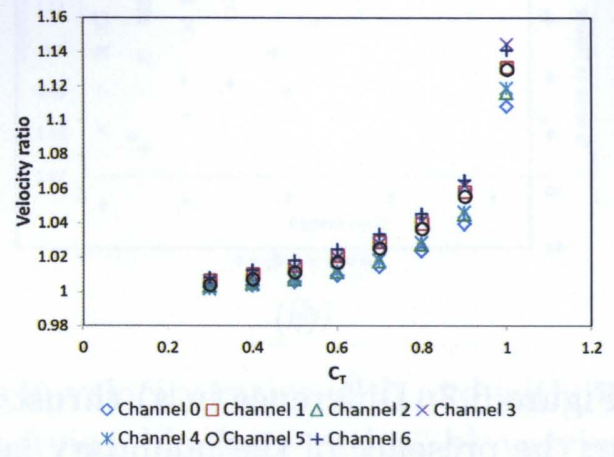

(b)

Figure 6.5: Comparison of the velocity ratios of the different channels a) in the presence of and b) without the presence of the boundary layer

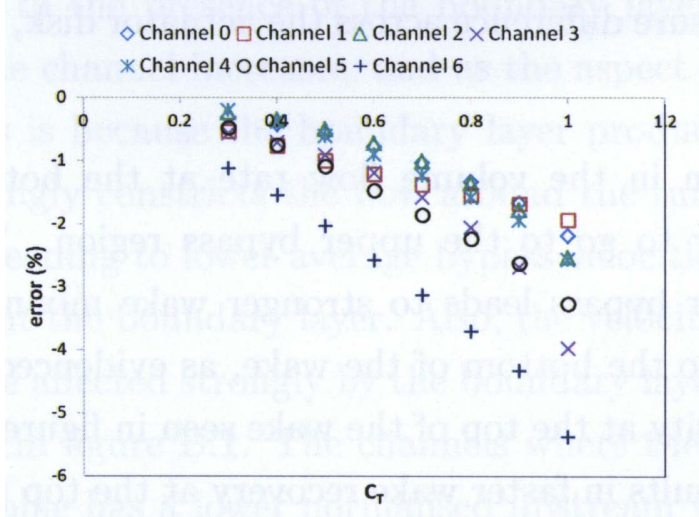

Figure 6.6: Difference in velocity ratios measured with and without the presence of a boundary layer 


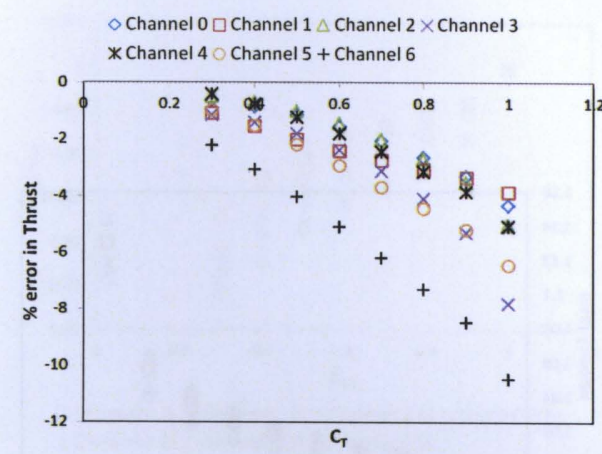

(a)

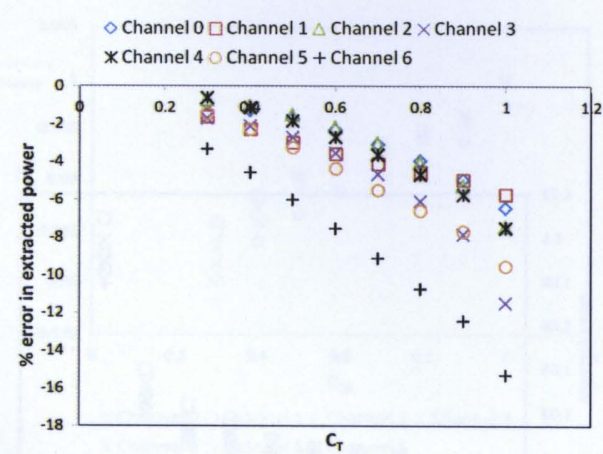

(b)

Figure 6.7: Difference in a) thrust and b) power extracted by a turbine due to the presence of the boundary layer

The decrease in performance is partially due to the reduction of the average bypass velocity as a result of the of the boundary layer constraining the flow below the turbine as seen in figure B.1 compared to figure B.4. This leads to a reduction of pressure drop in the bypass flow. Since pressure in the bypass flow and wake flow sufficiently upstream and downstream of the turbine has to be equalised, a reduction of pressure drop in the bypass flow leads to a reduction of pressure difference across the actuator disk, resulting in reduced thrust.

Also, a reduction in the volume flow rate at the bottom of the turbine causes more flow to go to the upper bypass region. This increased flow rate in the upper bypass leads to stronger wake mixing at the top of the wake compared to the bottom of the wake, as evidenced by higher levels of turbulence intensity at the top of the wake seen in figure B.2. The increased flow rate then results in faster wake recovery at the top portion of the wake, leading to the wake being pushed deeper into the boundary layer. This then results in the wake being entrained in the boundary layer and taking a longer time to recover. The entrained wake in turn reduces the amount of flow passing through the turbine, resulting in decreased thrust and power from the turbine. This effect is seen to be more pronounced when the turbine 


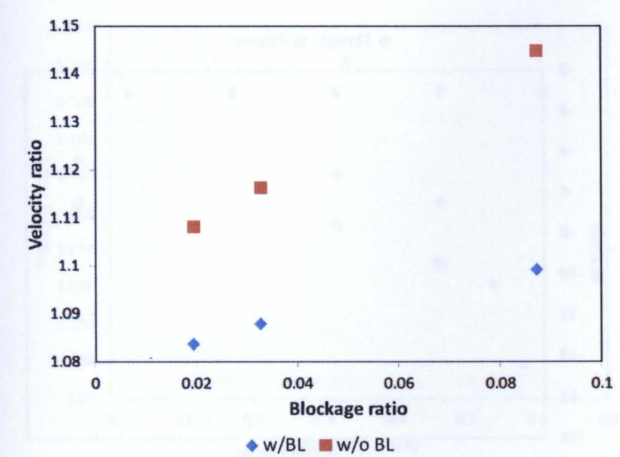

(a)

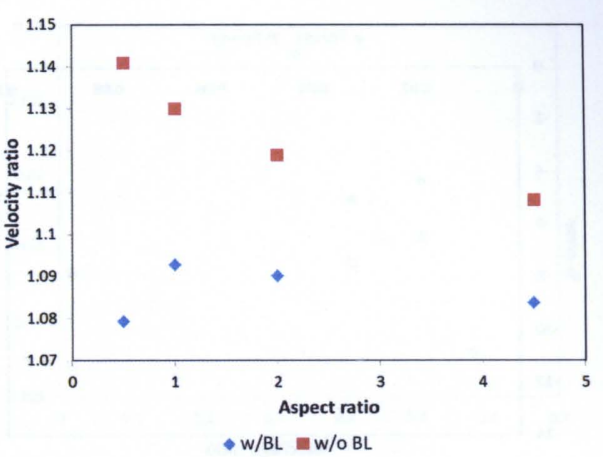

(b)

Figure 6.8: Comparison of the differences in velocity ratios with and without the boundary layer for channels of a) varying blockage ratios, b) varying aspect ratios

is located in shallower channels as compared to deeper channels.

Figure 6.8 compares the velocity ratios obtained in the channels with and without the presence of the boundary layer for different blockage ratios and aspect ratios for turbines operating at a thrust coefficient of 1 . Figure 6.9 then presents the corresponding difference in performance as a result of the differing velocity ratios. It is seen that the performance degradation of the turbine due to the presence of the boundary layer increases as the blockage ratio of the channel increases, and as the aspect ratio of the channel decreases. This is because the boundary layer produced by the lateral boundaries increasingly constricts the flow around the turbine as the channel gets narrower, leading to lower average bypass velocities as compared to the channels without the boundary layer. Also, the velocity upstream of the turbine is seen to be affected strongly by the boundary layer from the lateral boundaries as seen in figure B.1. The channels where the lateral boundary is closer to the turbine has a lower normalised upstream velocity compared to the channels where the lateral boundaries are further away.

The trend of the velocity ratio in the presence of the boundary layer with respect to changing blockage ratios and channel aspect ratio is observed to be similar to that obtained by Keogh et al. [117], who performed similar 


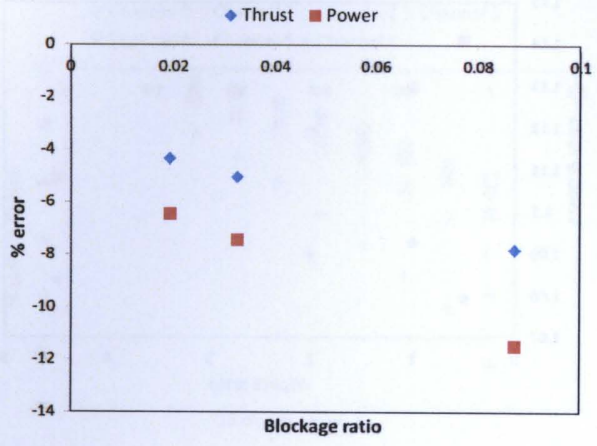

(a)

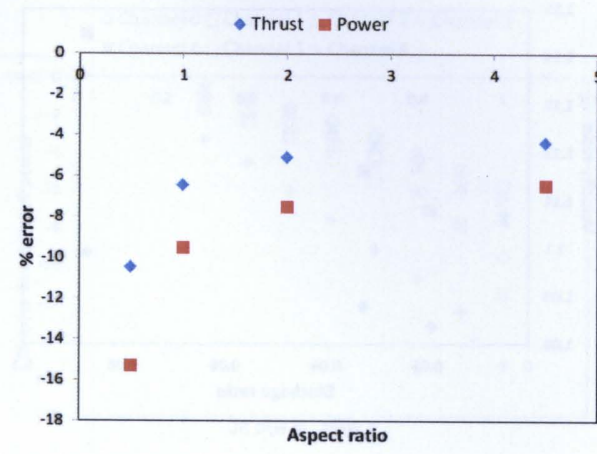

(b)

Figure 6.9: Difference in thrust and power generated by a turbine due to the presence of the boundary layer for channels of a) varying blockage ratios, b) varying aspect ratios

experiments in a flume. However, Keogh et al. [117] obtained a different value of the aspect ratio where the velocity ratio peaked. This could be due to the lateral and bottom walls of the flume producing different boundary layer profiles.

\subsubsection{Effect of flow properties}

From this section onwards, all simulations are performed with the channel boundaries set to the symmetry boundary condition. This is done to remove the uncertainty due to the presence of the boundary layer, as it is not known how it will be affected by the following properties. Results will also be shown at a $C_{T}$ of 1 for the results to be made clearer.

Figures $6.10 \mathrm{a}$ and $6.11 \mathrm{a}$ show the effect of the flow velocity and turbulence intensity on the velocity ratio measured from the experiments performed on Channel 0 respectively, while figures $6.10 \mathrm{~b}$ and $6.11 \mathrm{~b}$ show the effect of flow velocity and turbulence intensity on the maximum velocity reduction, $u_{3} / u_{0}$, in the wake of the actuator disk. Although not accounted for in analytical models, it is clear from the figures that the velocity ratios are affected by the flow properties. The significance of figures $6.10 \mathrm{~b}$ and $6.11 \mathrm{~b}$ will be discussed 


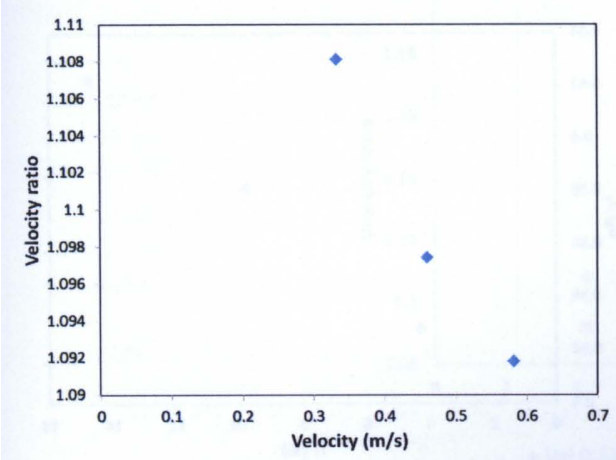

(a)

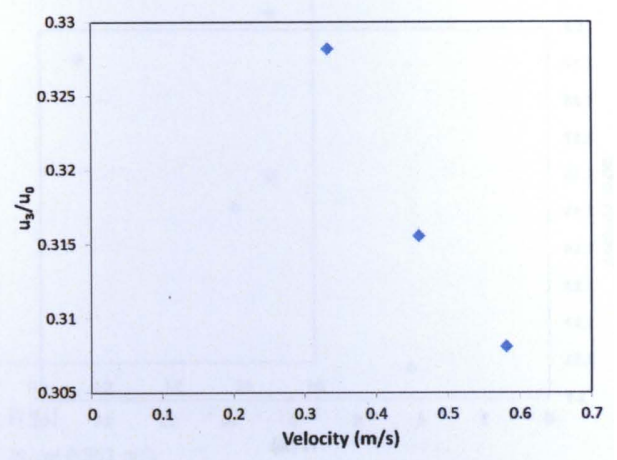

(b)

Figure 6.10: Changes of the a) velocity ratio, b) maximum velocity drop downstream of the turbine due to variation in the inlet velocity taken at thrust coefficient of 1.0

in Section 6.3.5.

Figure $6.10 \mathrm{a}$ shows that the velocity ratio decreased slightly with flow velocity, while studies [2] show that turbine performance increases with flow velocity until a certain value. It is seen from figure 6.11a that the velocity ratio increases with increasing turbulence intensity, whereas studies [94, 2] showed that the average turbine performance decreased with increasing turbulence intensity. The differences in behaviour could be due to the differences in how the flow interacts with the different devices and their wakes. Thrust generated by actuator disks is due to the reduction in pressure across the disk as a result of energy loss, while thrust in rotors are generated due to the forces on the rotor blades. The actuator disk removes energy from the flow through viscous drag and generation of heat, while rotors convert the energy in the flow into rotational kinetic energy which is transformed into electrical energy by a generator.

With actuator disks, the difference between the wake velocity and the bypass velocity increases as the velocity increases. This increase in difference 


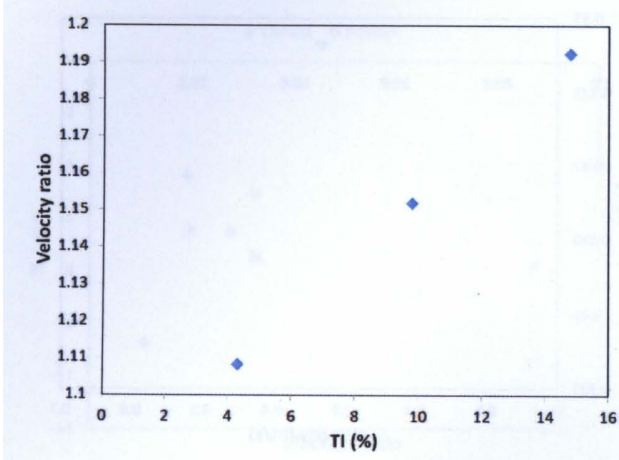

(a)

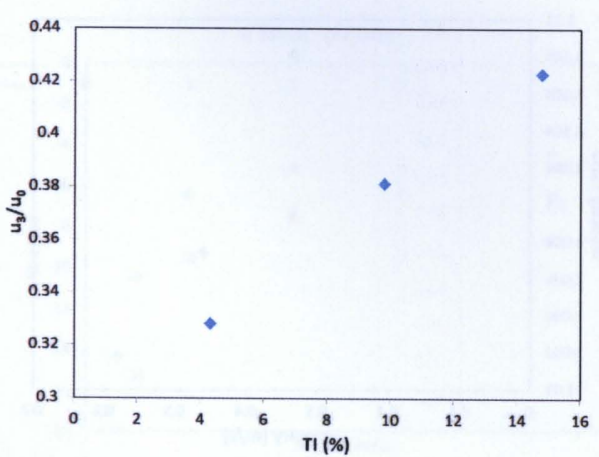

(b)

Figure 6.11: Changes of the a) velocity ratio, b) maximum velocity drop downstream of the turbine due to variation in the Turbulence Intensity taken at thrust coefficient of 1.0

leads to a stronger shear layer between the bypass flow and the wake flow, resulting in energy loss in the bypass flow. With a slower than expected bypass flow, the pressure reduction across the actuator disk will also decrease, leading to lower thrust levels when the thrust coefficient is being held constant. However, forces experienced by the rotor increases with velocity as the Reynolds number of the flow over the blades increases as well. This increase continues until Reynolds independence is reached.

The difference between the behaviour of the thrust predicted by the present actuator disk simulation and that by previous rotor experiments [94, 2] could be due to the difference in the relative turbulence length scale in the flow. Blackmore et al. [119] noted that the effect of turbulence on the device performance depended on the length scale of the turbulence and how it interacted with its wake. With a smaller turbulence length scale, increasing the turbulence intensity reduced the device performance, while the device performance improved with a large turbulence length scale. The present simulation has a turbulence length scale the size of the disk, which is considered large. This leads to the predicted thrust increasing with increasing turbulence intensity. The turbulence length scale in previous rotor experiments $[94,2]$ where the performance reduced with increasing turbulence intensity 


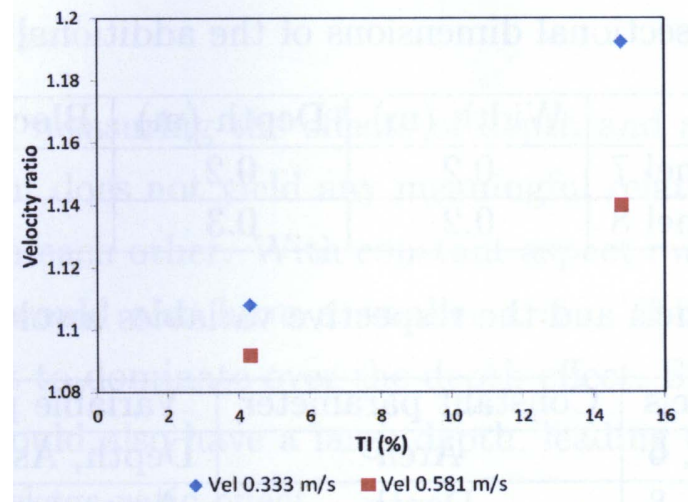

Figure 6.12: Interaction effects between Turbulence Intensity and velocity taken at thrust coefficient of 1.0

would then be smaller than the rotor diameter.

A similar study using the BEMT-AD approach to investigate the effects of velocity on the wake generated was performed in [54]. However, no details on the resulting performance of the turbine was reported.

Finally, figure 6.12 shows the combined effect of velocity and turbulence intensity. The different gradients of the 2 lines indicate that there is an interactive effect between the 2 quantities.

\subsubsection{Effect of channel geometry}

Inlet conditions for simulations performed in this section were adjusted such that the velocity and turbulent intensity at the turbine's location in all channels are as similar to each other as possible. This is to remove the effect of flow properties on the thrust produced by the actuator disk as mentioned in the previous section.

The only geometric quantity that is currently considered in analytical models is the blockage ratio, which is the ratio between the turbine swept area and the channel area. In addition to the blockage ratio, CFD studies conducted 
Table 6.2: Cross-sectional dimensions of the additional channels introduced

\begin{tabular}{|l|c|c|c|}
\hline & Width (m) & Depth $(\mathrm{m})$ & Blockage ratio \\
\hline Channel 7 & 0.2 & 0.2 & 0.196 \\
\hline Channel 8 & 0.2 & 0.3 & 0.131 \\
\hline
\end{tabular}

Table 6.3: Channels and the respective variables involved in each data set

\begin{tabular}{|c|c|c|}
\hline Channels & Constant parameter & Variable parameter \\
\hline $0,4,5,6$ & Area & Depth, Aspect Ratio \\
\hline $0,2,3,8$ & Depth & Area, Aspect Ratio \\
\hline $3,5,7$ & Aspect Ratio & Depth, Area \\
\hline
\end{tabular}

by Nishino and Willden [48] also considered the effect of the aspect ratio of the channel. In this study, channel depth is added as an additional parameter to be studied. Also, turbulent length scales at the inlet are assumed to be proportional to the channel depth for this study unless stated otherwise, so using channel depth can be seen a proxy to study the effects of the turbulent length scale on blockage effects. However, in real tidal sites, the turbulent length scales would be dependent on many factors. This effect is not studied by Nishino and Willden [48], who used constant TKE and dissipation at the inlet for all channels regardless of area and depth.

Trying to study each factor in isolation would lead to the realisation that varying each factor would cause another factor to vary as well. Thus, it would be impossible to isolate the effects of each factor. The approach taken ther was to hold each factor constant and allow the other two to vary, resulting in three sets of data. Two additional channels are introduced to ensure sufficient data points in each set of data. Table 6.2 shows the dimensions o the two additional channels, and table 6.3 presents the channels involved ir each data set.

Figures 6.13, 6.14, and 6.15 show the impact of each factor on the velocity ratio measured from CFD. It is seen that the two data sets for aspect ratic variation in figure 6.13 reveals the same behaviour, and that channel deptl and area have a strong co-relation with each other, as seen in figures 6.1 . 
and 6.15 respectively.

On the other hand, measuring the effects of depth and area while keeping aspect ratio constant does not yield any meaningful relationship as the two factors interact with each other. With constant aspect ratio, a channel with a shallower depth would also have a smaller area. This would cause the blockage ratio effect to dominate over the depth effect. Similarly, a channel with a large area would also have a large depth, leading to the depth effect dominating the blockage ratio effect.

Simulations were also performed on the channels with constant area with constant turbulent length scale specified at the inlet, with the results seen in figure 6.16. It is seen that the velocity ratio decreases with increasing aspect ratio even as the turbulent length scale at the inlet was held constant for all channels. This is in contrast to the results published by Nishino and Willden [48], where it was showed that a turbine placed in a higher aspect ratio channel produced a higher thrust than when it were to be placed in a lower aspect ratio. This could be due the fact that the rate of decay of turbulent intensity is different for the two channels compared. Thus, the turbulent intensity at the turbine's location would be different, affecting the accuracy of the result. Contour plots of the normalised velocity, turbulence intensity and pressure for the different cases shown in figure 6.16 can also be found in Appendix B, where it is seen that there is actually very little difference between the cases where the turbulent length scale varies with depth and the cases with constant turbulence length scale.

For the constant dissipation case, it is noticed that results for the channels with aspect ratio 2 and $1 / 2$ should be similar to each other, yet they produced different velocity ratios. Since the channel depth is the only other varying parameter, it can be concluded that the physical depth of the channel has an effect on the thrust produced by a turbine, instead of just being a proxy for the turbulent length scale as assumed in the beginning of the section. However, this could also be due to the fact that the rate of decay 


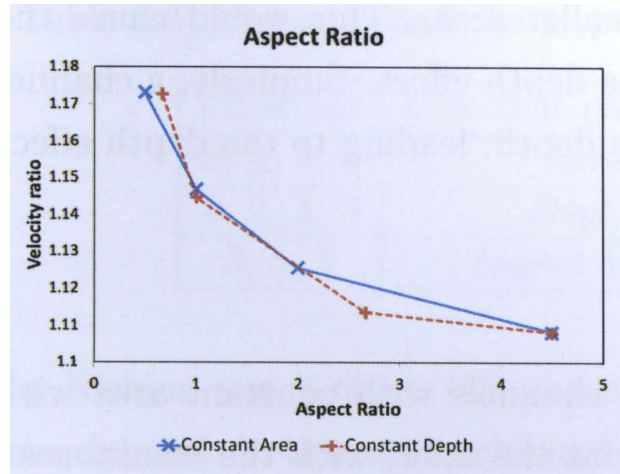

Figure 6.13: Effect of varying the aspect ratio of the channel on the measured velocity ratio taken at thrust coefficient of 1.0

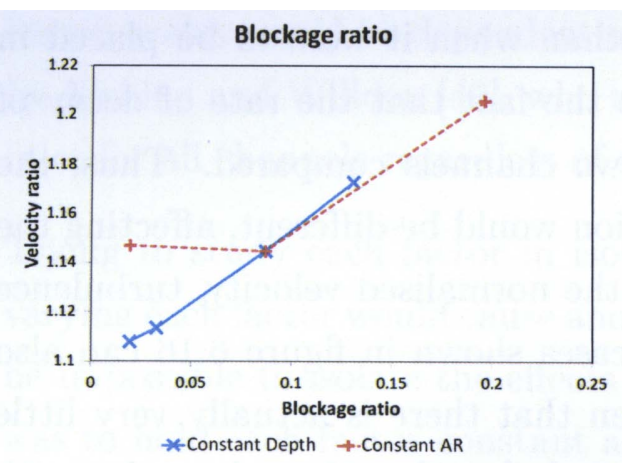

Figure 6.15: Effect of varying the blockage ratio of the channel on the measured velocity ratio taken at thrust coefficient of 1.0

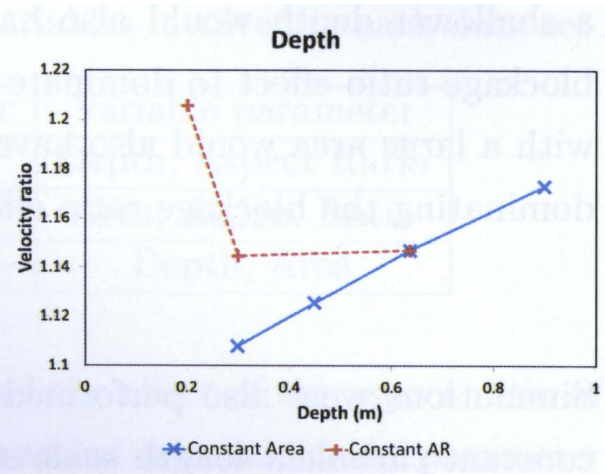

Figure 6.14: Effect of varying the depth of the channel on the measured velocity ratio taken at thrust coefficient of 1.0 


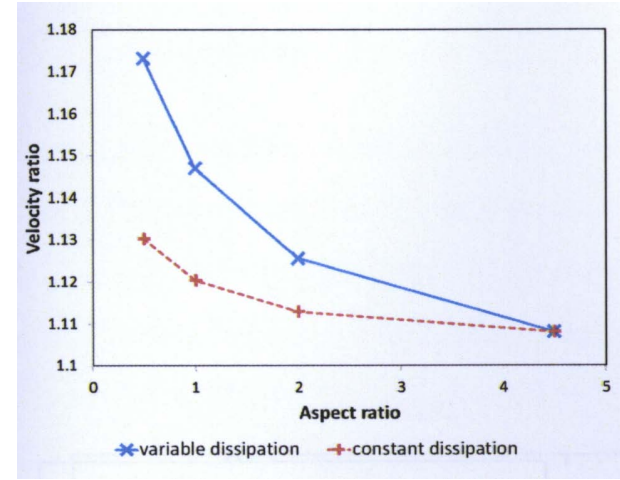

Figure 6.16: Comparison of the effects of dissipation on the velocity ratio with respect to aspect ratio variations

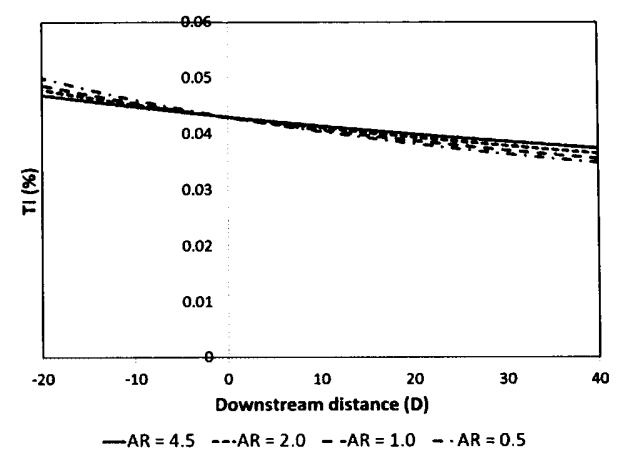

Figure 6.17: Comparison of the decay rate of turbulence intensity in the channels of different aspect ratios

of turbulent intensity in the four channels are different. This is illustrated in figure 6.17 for channels without the presence of the turbine.

\subsubsection{Wake recovery}

The wake recovery of the the turbine in response to the different geometric quantities is also studied. Figure 6.18 shows the wake recovery of the turbine for the channels with constant depth and varying blockage ratio, while figure 6.19 shows the wake recovery for the channels with constant area and different depth. The presented results are for the case where the boundary layer is absent.

Figure 6.18 shows that wake recovery is affected by the blockage ratio of the channel, despite the turbulence intensity being the same for all cases. Wake recovery in the far wake is seen to increase linearly with increasing blockage ratio for the blockage ratios considered, whereas the near wake does not seem so affected by the blockage ratio. This could be due to the fact that stronger mixing of the wake as a result of larger blockage ratios cause the wake to recover faster. This means that it is possible to shorten the distance between 


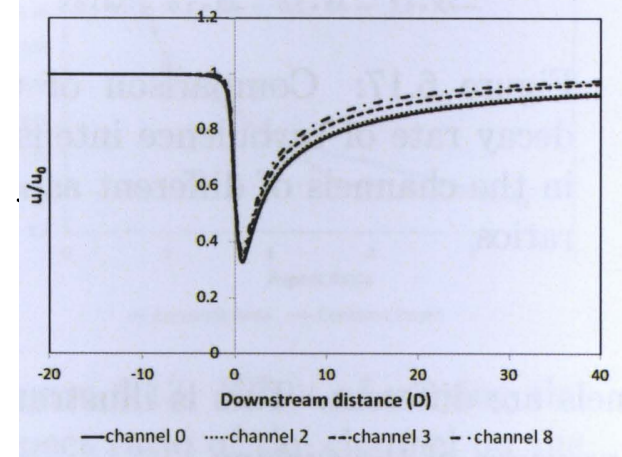

(a)

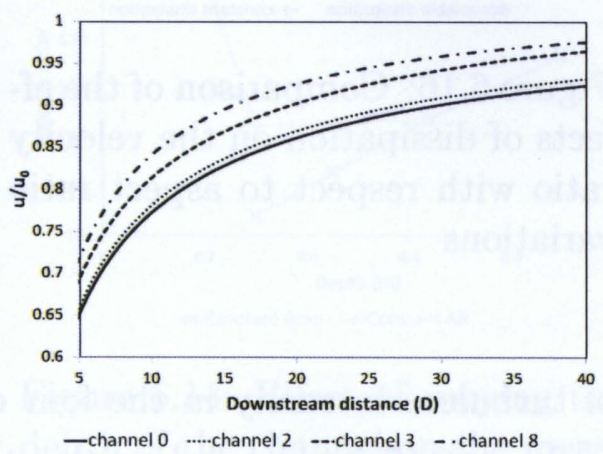

(b)

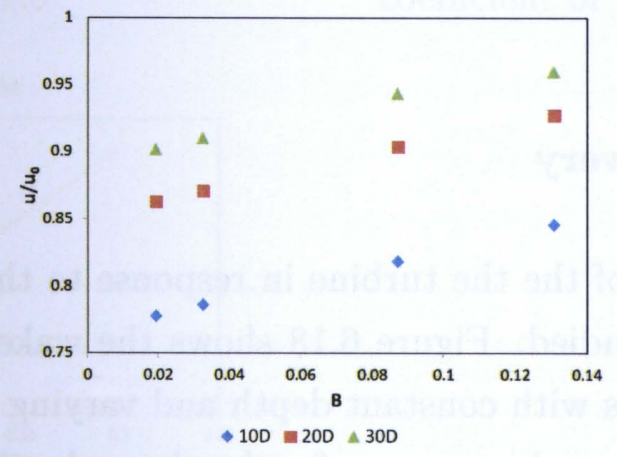

(c)

Figure 6.18: Comparison of the wake generated in channels of constant depth and varying area for a) the full length of the domain, b) from 5D onwards, c) various downstream locations 


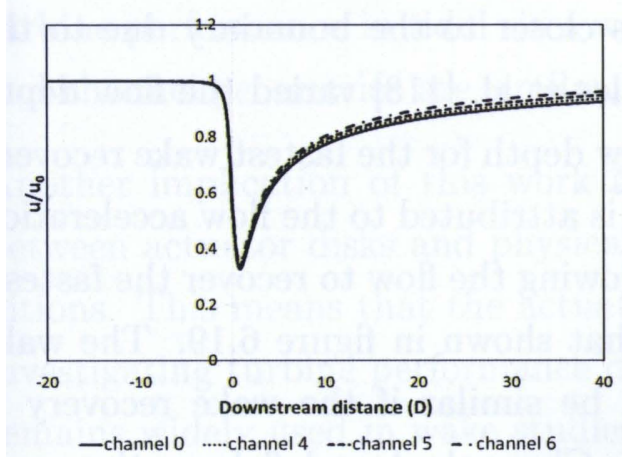

(a)

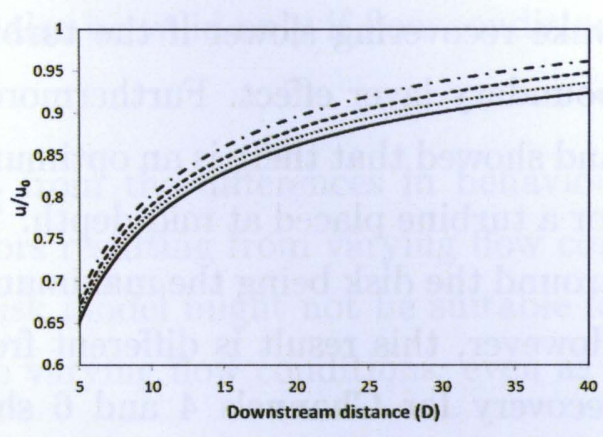

(b)

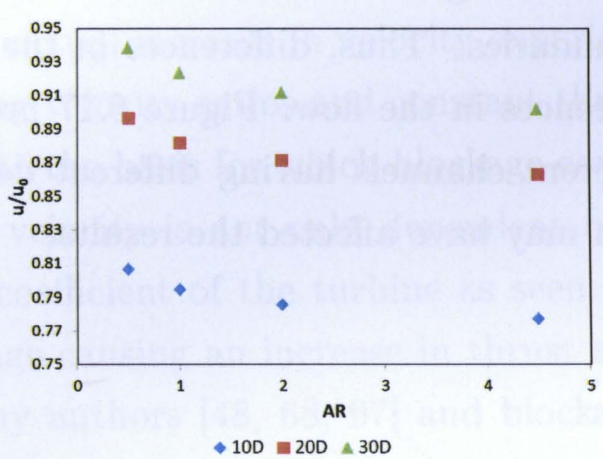

(c)

Figure 6.19: Comparison of the wake generated in channels of constant area and different depth for a) the full length of the domain, b) from 5D onwards, c) various downstream locations 
rows in multi-row arrays when operating in higher blockage environments.

Figure 6.19 suggests that the wake recovery is also affected by the depth of the channel, with the wake recovering faster as the depth increases. This is in partial contrast to the results of Myers and Bahaj [97] and Giles et al. [118], who performed flume experiments and showed that the wake recovery of a turbine depended on its proximity to the flow boundaries, with the wake recovering slower if the turbine is closer to the boundary due to the boundary layer effect. Furthermore, Giles et al. [118] varied the flow depth and showed that there is an optimum flow depth for the fastest wake recovery for a turbine placed at mid-depth. This is attributed to the flow acceleration around the disk being the maximum, allowing the flow to recover the fastest. However, this result is different from that shown in figure 6.19. The wake recovery for Channels 4 and 6 should be similar if the wake recovery is influenced by its boundary proximity. Channels 4 and 6 have the same channel dimensions, meaning that the turbines are the same distance away from the closest boundaries. Thus, differences in the wake recovery could then be due to differences in the flow. Figure 6.17 presents the turbulence intensity in the different channels having different decay rates due to the varying depth, which may have affected the results.

\subsubsection{Discussion}

Considering the constant depth data-set in figure 6.15 , it is seen that there is a linear relationship between blockage ratio and the velocity ratio predicted from CFD. This is in contrast to studies which suggest that blockage ratio effects below a value of $10 \%$ are negligible [68, 78]. Also, assuming the linear relationship holds and extrapolating to the $\mathrm{Y}$-axis, it is seen that the line does not intercept at 1 . This implies that even in a truly blockage free condition, thrust and power produced by the turbine will still be of some percentage higher than what is predicted in equation 3.5. Reasons for this behaviour is explored in Chapter 7. However, the current observation is only valid for the numerical model used in this study, and more studies have to 
be conducted to establish the extent of this observation.

One implication of this study as a whole is that the usage of current blockage correction models to predict a turbine's performance should be at best adopted as a guideline, since there are many factors that influences the performance of a turbine beyond the blockage ratio of the channel. Using performance data measured in a cavitation tunnel or tow tank to predict turbine performance in tidal sites would also be valid only if flow conditions and channel geometries are similar.

Another implication of this work arises from the differences in behaviour between actuator disks and physical rotors resulting from varying flow conditions. This means that the actuator disk model might not be suitable for investigating turbine performance due to varying flow conditions, even as it remains widely used in wake studies.

Results in the previous sections show that the increase in thrust caused by blockage is due to a virtual increase in velocity experienced by the turbine, as seen by the varying velocity ratios and constant thrust coefficients. This behaviour is similar to the basis for which blockage correction is carried out [71]. The increased velocity is not only dependent on the blockage ratio, but also the thrust coefficient of the turbine as seen in figure 6.5. This is in contrast to blockage causing an increase in thrust and power coefficients as presented by many authors $[48,68,67]$ and blockage correction models [5]. Although the end result for both methods is to cause an increase in performance of the turbine, the mechanism by which it happens is however very different. Presenting the effect of increased blockage as an increase in thrust and power coefficients suggests that blockage causes the characteristics of the device to change, even if the device settings remain unchanged. It might also give problems for researchers who might want to use wake models to predict the performance of a farm. A thrust coefficient of more than unity might occur if the turbine is placed in a highly blocked channel, resulting in the wake velocity having imaginary values if the actuator disk model is used. However, presenting the effect of blockage as an increase in velocity 
experienced allows the device characteristics to remain constant regardless of the environment it is placed in. But, in practice, it might be difficult to determine the increased velocity with the method applied in this study as it is almost impossible to determine the velocity at the turbine plane when using a physical turbine.

Although it is not the main subject of study in this chapter, it is seen that the conventional actuator disk theory fails in predicting the maximum velocity drop in the wake of the turbine. When operating at a $C_{T}$ of 1 , conventional actuator disk theory predicts that the minimum wake velocity should reduce to zero. However, Figures $6.10 \mathrm{~b}$ and $6.11 \mathrm{~b}$ clearly show that the maximum velocity drop in the wake is not to zero even in a relatively unblocked channel. However, this observation agrees with empirical formulae [11, 41] that correct the thrust produced by an actuator disk operating at high axial induction factors. An axial induction factor of less than 0.5 is returned if the freestream $C_{T}$ of 1 is put into the empirical formulae, resulting in a wake velocity that is more than zero.

This could also be due to the fact that the actuator disk theory was derived while assuming free-stream conditions, leading to the free-stream velocity being traditionally used as a reference to calculate the axial induction factor. However, a non-zero velocity in the wake of the turbine can be predicted if the increased velocity is treated as the reference velocity instead.

Finally, it is seen that the performance and wake recovery of the actuator disk is affected by the flow and geometrical properties of the channel. Also, it is shown that the geometrical properties of the channel can affect the flow properties of the channel. In order to solely study the effect of blockage on the turbine performance, the effect of flow properties on the turbine performance has to be reduced as much as possible.

The blockage ratio of the channel can be varied by either varying one dimension of the channel while keeping the other constant, or varying both dimensions of the channel while keeping the aspect ratio of the channel constant. It is shown in figure 6.17 that the turbulence decay rate is different 
for channels with different depths, possibly causing the difference in performance of the turbine seen in figure 6.16. In order to solely study the effect of blockage on the performance and wake recovery of the turbine, the channel depth should be held constant while varying the width in order to eliminate the possibility of the turbulence decay rate affecting the turbine performance. This will be implemented throughout the rest of the thesis.

\subsection{Concluding remarks}

This chapter presents a series of CFD simulations done to study the effect of various factors on the thrust of a tidal turbine. It was found that the presence of the boundary layer reduced the thrust of the tidal turbine due to the reduced bypass flow velocity. Flow velocity and turbulence intensity were also found to influence the thrust of a turbine. Thrust increased with increasing turbulence intensity while the rate of thrust increase decreased with increasing velocity. Aspect ratio, channel depth and channel area were also used to study the effect of channel geometry on the turbine thrust. Thrust was found to increase linearly with increasing blockage ratio when the channel depth was kept constant. Depth of the channel was also found to have an effect on the performance of the turbine, possibly due to differences in the decay of the turbulent quantities in the flow.

Wake recovery of the turbine was also studied. The recovery of the wake was found to increase linearly with increasing blockage ratio when the channel depth was kept constant. Channel depth was also found to have an effect on the wake recovery, also possibly due to the differences in the decay of the turbulent quantities in the flow.

Thus, it is recommended to keep the channel depth constant and vary the channel width when studying the effects of blockage on the turbine, so as not to let unintended flow effects to affect the result. The limitations of current blockage correction models were also made clear in light of the various factors affecting turbine performance, and will be studied in more detail in the next 
chapter. 


\section{Chapter 7}

\section{Analytical prediction of the performance and wake recovery of a single tidal turbine under blocked conditions}

This chapter investigates the ability of analytical models to accurately predict the blockage effect of turbines by comparing their predictions with CFD simulations performed in the previous chapter. Three blockage correction models were evaluated for their ability to predict the performance of a turbine in blocked conditions, while two wake prediction models were evaluated for their ability to predict the wakes generated by CFD. Validation with experimental results was performed for the blockage correction models. The blockage model used by Bahaj et al. had the best match with the performance predicted by CFD, while the Jensen wake model is recommended for predicting the wake in blocked conditions. 


\subsection{Introduction}

As more tidal turbine deployments are being planned, it is important to be able to predict the effects of blockage on the performance of tidal turbines in order to make better design and planning decisions. Most of the current blockage models are used to correct the performance of the turbine obtained in blocked conditions to achieve the free-stream equivalent performance. Bahaj et al. [1] used a blockage model developed by Barnsley and Wellicome [73], who modified a form of Glauert's model [71] to correct data from a tidal turbine experiment. This was subsequently adopted by other authors to correct data obtained from water tunnel experiments $[61,8,74]$. Whelan et al. [76] modified the actuator disk model to account for the variation in water surface due to the reduction in pressure in the wake of the turbine, and used Maskel's theory [75] to correct their data obtained from water tunnel experiments. Garrett and Cummins [5] derived an expression that predicts the performance of a single turbine in a blocked environment. This was expanded in $[83,84]$ to describe the performance of an array partially blocking the channel.

It is seen that the models mentioned above often appear on their own. As such, there is no information on how the performance of the different models compare with each other. Accuracy of the models are also unknown as there are currently no method to directly measure the free-stream performance of the turbines.

This chapter attempts to quantify the accuracy of the different blockage correction and wake models by comparing them against each other, as well as results from the CFD simulations presented in the previous chapter. 


\subsection{Analytical models}

\subsubsection{Blockage correction models}

The models by Garrett and Cummins [5], Whelan et al. [76], and that used by Bahaj et al. [1] presented in section 3.3 will be used for comparison in this chapter. Velocity quantities in this subsection is the same as that presented in figure 3.3 .

The original models by Whelan et al. and Bahaj et al. were derived to predict the free-stream performance given the performance under blocked conditions. Thus, some modifications are needed in order to make predictions on the performance under blocked conditions given the free-stream performance. The modified model of Whelan et al. is presented in equations 7.1 to 7.4 , while the modified model of that used by Bahaj et al. is presented in equations 7.5 to 7.8 .

$$
\begin{gathered}
\tau=\frac{u_{4}}{u_{0}}=\frac{1-\alpha+\sqrt{B-2 \alpha B+\alpha^{2}\left(1-B+B^{2}\right)}}{1-B} \\
\frac{4\left(1-\sqrt{1-C_{T}}\right)}{1+\sqrt{1-C_{T}}}=\frac{\tau^{2}-\alpha^{2}}{\delta^{2}} \\
\alpha=\frac{u_{3}}{u_{0}} \\
\delta=\frac{u_{1}}{u_{0}}=\frac{\alpha(\tau-1)}{B(\tau-\alpha)} \\
\frac{u_{1}}{u_{3}}=\frac{-1+\sqrt{1+B\left(\left(u_{4} / u_{3}\right)^{2}-1\right)}}{B\left(u_{4} / u_{3}-1\right)}
\end{gathered}
$$




$$
\begin{gathered}
\frac{u_{0}}{u_{3}}=\frac{u_{4}}{u_{3}}-B \frac{u_{1}}{u_{3}}\left[\frac{u_{4}}{u_{3}}-1\right] \\
\frac{u_{0}}{u_{3}}=1 / \sqrt{\frac{f\left(u_{1} / u_{0}\right)^{2}}{\left[\left(u_{4} / u_{3}\right)^{2}-1\right]}} \\
f=\frac{4\left(1-\sqrt{1-C_{T}}\right)}{1+\sqrt{1-C_{T}}}
\end{gathered}
$$

Equations 7.6 and 7.7 are solved iteratively until a value of $u_{0} / u_{3}$ is found to satisfy both equations. $C_{T}$ in equations 7.2 and 7.8 refers to the free-stream thrust coefficient. The MATLAB code for the implementation of equations 7.5 to 7.8 can be found in Appendix D.

\subsubsection{Wake models}

Similar to section 7.2.1, velocity quantities in this subsection is the same as that presented in figure 3.3 .

Despite its shortcomings, the Jensen model is a very popular wake model due to its simplicity. It has been modified and used extensively in wind farm wake predictions $[120,121]$, and recently in simple tidal farm arrangements [122]. The equations describing the Jensen model in its original form can be found in [122]. In this study, the model has been modified for use in blocked conditions, and is presented in the following equations.

$$
\begin{gathered}
r=r_{1}+k x \\
r_{1}=r_{0} \sqrt{0.5 \frac{\left(1+u_{3} / u_{0}\right)}{u_{3} / u_{0}}}
\end{gathered}
$$




$$
\begin{gathered}
\pi r_{0}^{2} u_{w 0}+\pi\left(r^{2}-r_{1}^{2}\right) U_{0}=\pi r^{2} u_{w} \\
u_{w 0}=\frac{u_{3}}{u_{0}}
\end{gathered}
$$

where $x$ represents the distance behind the turbine, $r_{0}$ represents the radius of the turbine, $r_{1}$ represents the radius of the wake after the initial wake expansion, and $r$ represents the radius of the wake at a distance $x$ behind the turbine. $u_{0}$ represents the magnitude of the free-stream velocity, $u_{w 0}$ represents the velocity in the wake after the initial wake expansion, and $u_{w}$ represents the velocity in the wake when the wake has a radius of $r$.

The wake model by Bastankhah and Porté-Agel [9] builds upon the model by Frandsen et al. [123] by assigning a Gaussian wake profile within the wake boundaries, instead of a uniform velocity profile. The equations describing the model in it's original form can be found in [9]. It was also derived under free-steam conditions, and is similarly modified for use under blocked conditions for this study. The following equations presents the modified model.

$$
\begin{gathered}
\frac{\Delta u}{u_{0}}=\left(1-\sqrt{1-\frac{C_{T, B}}{8\left(k^{*} x / d_{0}+\varepsilon\right)^{2}}}\right) \times \\
\exp \left(-\frac{1}{2\left(k^{*} x / d_{0}+\varepsilon\right)^{2}}\left\{\left(\frac{z-z_{h}}{d_{0}}\right)^{2}+\left(\frac{y}{d_{0}}\right)^{2}\right\}\right) \\
\varepsilon=\frac{\left(1+u_{3} / u_{0}\right)}{6.6\left(1-u_{3} / u_{0}\right)} \sqrt{\beta} \\
\beta=\frac{A_{a}}{A_{0}}=0.5 \frac{\left(1+u_{3} / u_{0}\right)}{u_{3} / u_{0}}
\end{gathered}
$$


Table 7.1: Cross sectional dimensions of the channels tested

\begin{tabular}{|c|c|c|}
\hline & Width $(\mathrm{m})$ & Depth $(\mathrm{m})$ \\
\hline Channel 0 & 1.35 & 0.3 \\
\hline Channel 2 & 0.8 & 0.3 \\
\hline Channel 9 & 0.4 & 0.3 \\
\hline Channel 3 & 0.3 & 0.3 \\
\hline Channel 8 & 0.2 & 0.3 \\
\hline
\end{tabular}

where $\triangle u / u_{0}$ represents the velocity deficit in the wake. $d_{0}$ represents the diameter of the turbine, and $A_{a}$ is the area of the wake immediately after initial wake expansion. $u_{3} / u_{0}$ is calculated from the blockage correction models presented in section 7.2.1. $x$ represents the stream-wise Cartesian coordinates, while $y$ and $z$ represents the lateral and vertical Cartesian coordinates.

Equation 7.14 is a function that is found to provide satisfactory performance for blockage ratios up till 0.3 , as the original form could not work with equation 7.15 .

$k$ and $k *$ are variables that determine the spreading of the wake for Jensen model and the model by Bastankhah and Porté-Agel [9] respectively, and are required to be tuned in order to match the wake predictions.

\subsection{Methodology}

Five of the channels previously used in Chapter 6 are chosen to be compared with the analytical equations and listed according to blockage ratio in Table 7.1. The channels are chosen as all of them have the same depth, as it is shown that channels with the same area but different depths produced different results. The velocity ratio, $\gamma$, defined in equation 4.6 is compared across all the different models used.

$\gamma$ for the model proposed by Garrett and Cummins [5] can be calculated from equation 3.26, while $\gamma$ for the method proposed by Whelan et al. [76] is 


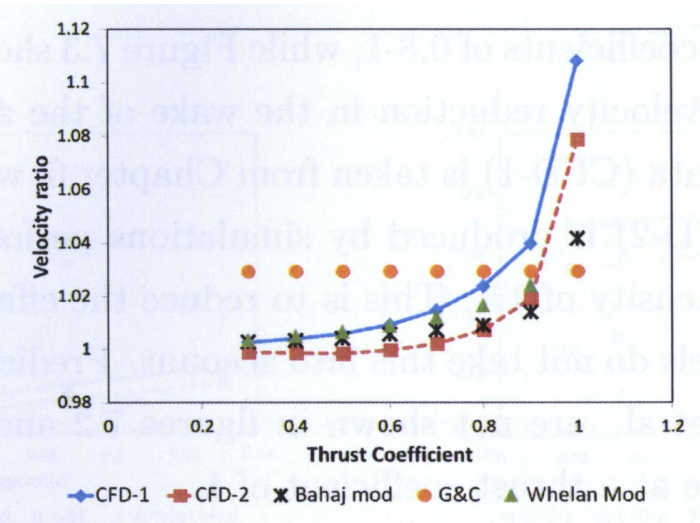

Figure 7.1: Comparison of the different analytical models with CFD simulations for different thrust coefficients. CFD-1 refers to the data set taken from Chapter 6, CFD-2 refers to the simulations performed with lower inlet turbulent intensity

just $\tau$ in equation 7.1. $\gamma$ for the modified model of Barnsley and Wellicome [73] can be calculated using equations 4.3 and 4.6.

\subsection{Results and discussion}

\subsubsection{Performance prediction}

Figure 7.1 shows the comparison between the velocity ratio predicted by the blockage correction models by Garrett and Cummins [5], Bahaj et al. [1] and Whelan et al. [76] and that predicted by CFD for different thrust coefficients obtained for Channel 0 . It is seen that with the exception of the model by Garrett and Cummins [5], the different analytical models are able to match the velocity ratios with a good degree of agreement for thrust coefficients from 0.3-0.9. The main differences between the different models can be seen when predicting the velocity ratio for a thrust coefficient of 1 .

Figure 7.2 shows the comparison of the velocity ratios predicted by CFD versus that predicted by the different analytical models for different blockage 
ratios with thrust coefficients of $0.8-1$, while Figure 7.3 shows the comparison of the maximum velocity reduction in the wake of the actuator disk. The first set of CFD data (CFD-1) is taken from Chapter 6, while the second set of CFD data (CFD-2) is produced by simulations performed with a lower inlet turbulent intensity of $1 \%$. This is to reduce the effect of wake mixing, as analytical models do not take this into account. Predictions by the model used by Whelan et al. are not shown in figures 7.2 and 7.3 as the model could not converge at a thrust coefficient of 1 .

It is seen that the variation of the velocity ratio and velocity reduction predicted by CFD are less sensitive to the variation in blockage ratios as compared to the analytical models. The effect of decreasing the turbulent intensity of the free-stream flow is a downward shift of both the velocity ratio and velocity reduction lines in figures 7.2 and 7.3 while the gradient remains almost constant. It is seen that the model by Bahaj et al. has the best agreement with the CFD model for the range of blockage ratios tested, while the model by Garrett and Cummins [5] had the worst agreement.

The models used by Bahaj et al. [1] and Whelan et al. [76] are both derived using the same considerations. As such, the velocity reduction predicted by both the models are almost identical. However, the predicted velocity ratio varies between them as the measured quantity is different. It is seen that using the theory by Maskell [75] results in a higher prediction of the velocity ratio, which over predicts the CFD results at higher blockage ratios.

Differences in behaviour between the CFD results and the analytical models could be explained by the differences in flow around the turbine as predicted by CFD and that assumed by the analytical models. At lower blockage ratios, the higher than expected thrust can be explained by the presence of local flow acceleration around the turbine, which is shown in figure 7.4. This differs from the flow assumed by analytical models, which presume uniform flow in the bypass region. According to the actuator disk theory, this means that pressure in the bypass flow is lower than predicted, leading to a higher than predicted thrust. This could also explain the fact that the trend lines 


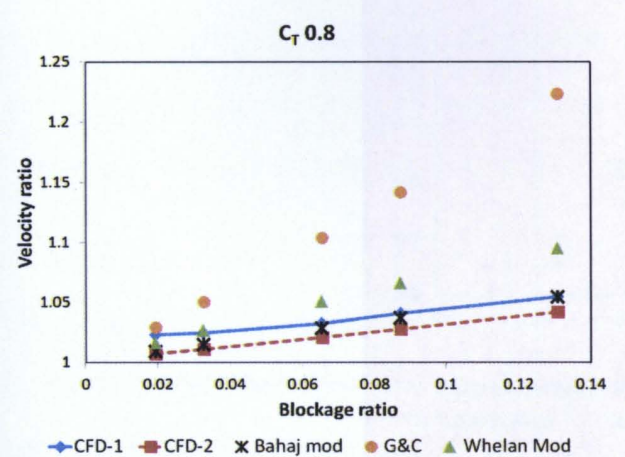

(a)

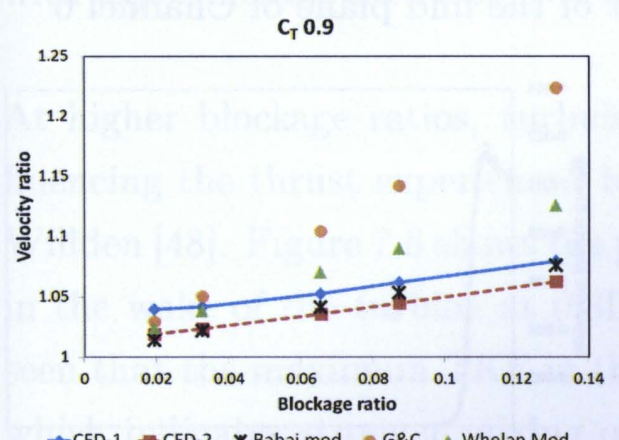

(b)

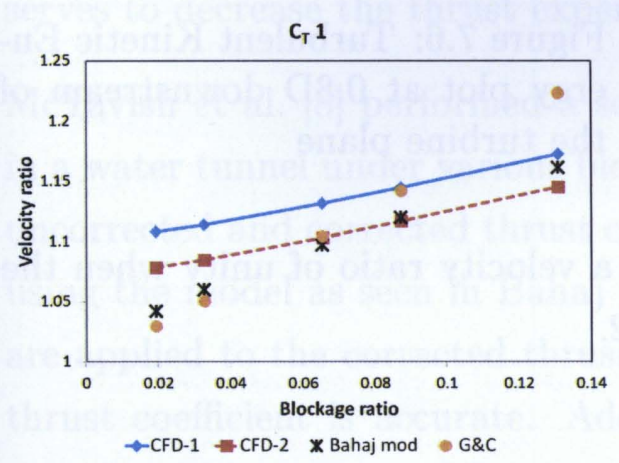

(c)

Figure 7.2: Variation of the velocity ratio predicted by the different models with increasing blockage ratio for thrust coefficients of $0.8,0.9$ and 1.0 respectively

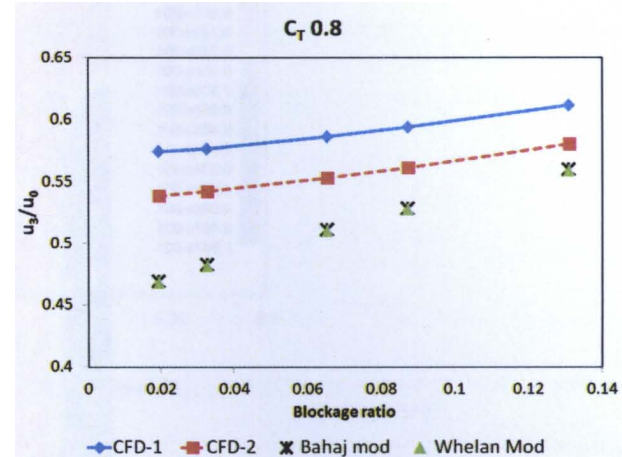

(a)

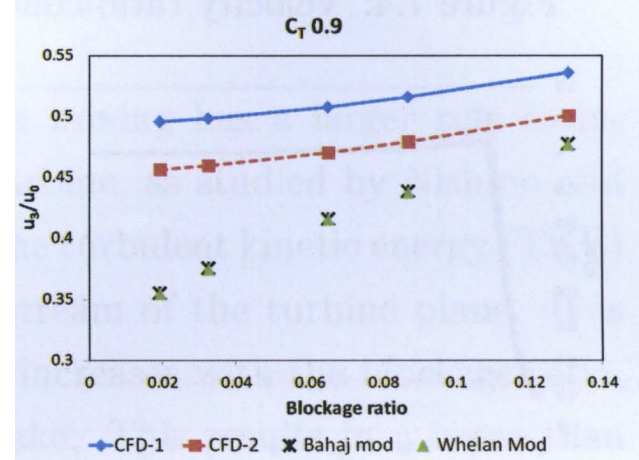

(b)

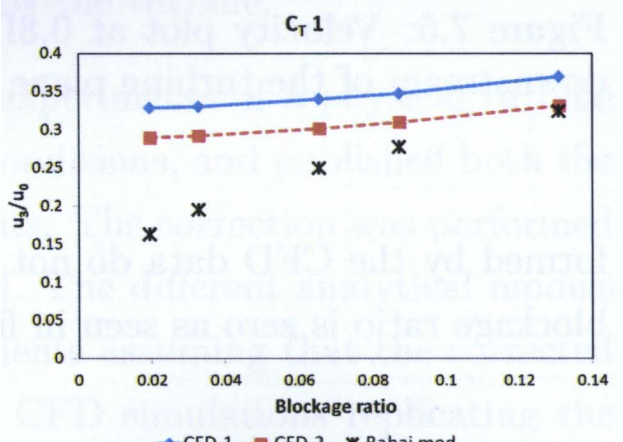

(c)

Figure 7.3: Variation of the maximum velocity reduction predicted by the different models with increasing blockage ratio for thrust coefficients of $0.8,0.9$ and 1.0 respectively 


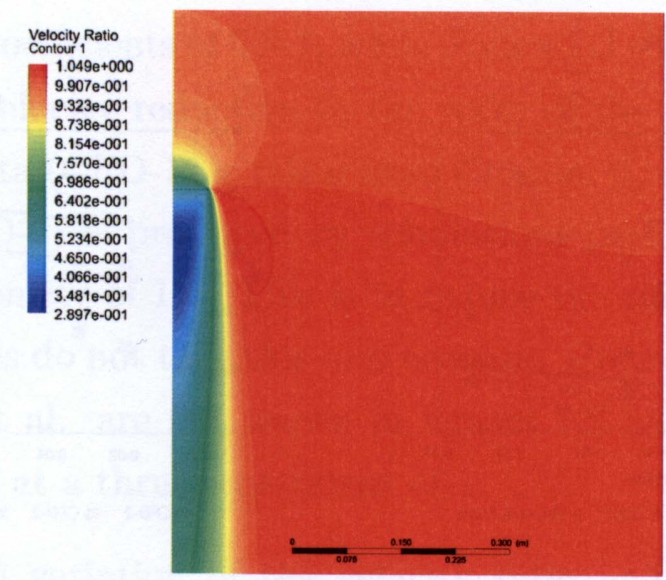

Figure 7.4: Velocity ratio contour plot of the mid plane of Channel 0

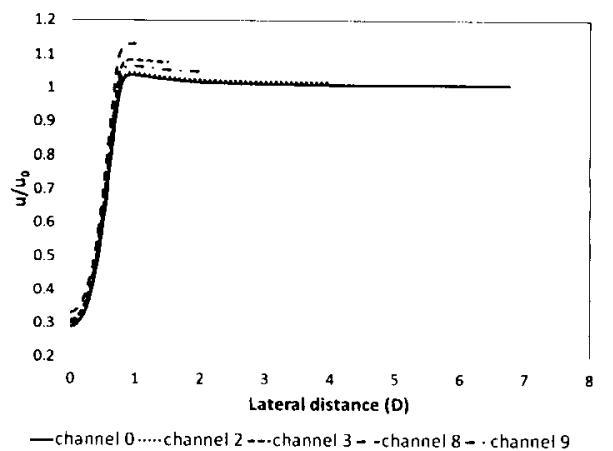

Figure 7.5: Velocity plot at $0.8 \mathrm{D}$ downstream of the turbine plane

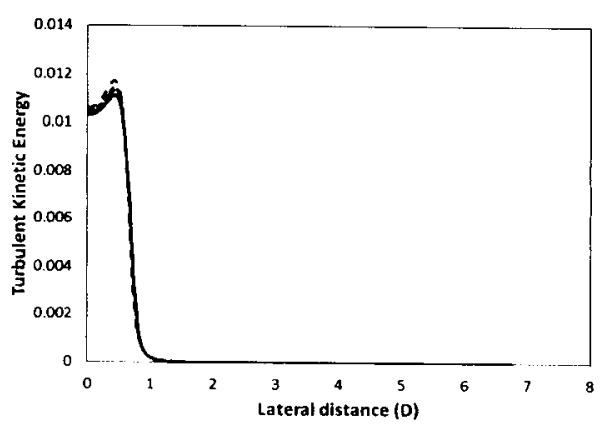

-channel 0.....channel 2---channel 3--channel 8-.channel 9

Figure 7.6: Turbulent Kinetic Energy plot at $0.8 \mathrm{D}$ downstream of the turbine plane

formed by the CFD data do not predict a velocity ratio of unity when the blockage ratio is zero as seen in figure 7.2.

Figure 7.5 shows the lateral velocity profile of all the channels for the low turbulent intensity case taken at $0.8 \mathrm{D}$ downstream of the turbine plane, where it is observed that the minimum wake velocity occurs. It is seen that the local flow acceleration occurs in all channels, regardless of the blockage ratio. However, this effect is seen to be less pronounced as the blockage ratio increases. 


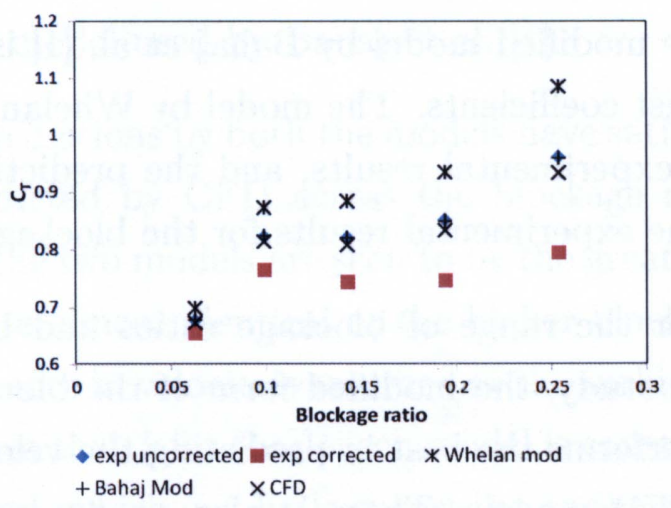

Figure 7.7: Thrust coefficients predicted by the different models compared with experimental data from McTavish et al. [8]

At higher blockage ratios, turbulent wake mixing has a larger role in influencing the thrust experienced by the turbine, as studied by Nishino and Willden [48]. Figure 7.6 shows the plot of the turbulent kinetic energy (TKE) in the wake of the turbine at $0.8 \mathrm{D}$ downstream of the turbine plane. It is seen that the maximum TKE in the wake increases with the blockage ratio, which indicates stronger mixing of the wake. This results in a lower than expected bypass velocity and a higher than expected wake velocity, which serves to decrease the thrust experienced by the turbine.

McTavish et al. [8] performed a series of experiments of a physical turbine in a water tunnel under various blockage conditions, and published both the uncorrected and corrected thrust coefficients. The correction was performed using the model as seen in Bahaj et al. [1]. The different analytical models are applied to the corrected thrust coefficients assuming that the corrected thrust coefficient is accurate. Additional CFD simulations replicating the experimental set-up and flow conditions are also performed, replacing the rotors with actuator disks of similar size. Results of all models are compared against the experimental results and presented in figure 7.7. Results for the model by Garrett and Cummins [5] is left out as its behaviour is seen to be different from the other models. 
It is seen that the modified model by Bahaj et al. [1] is able to recover the experimental thrust coefficients. The model by Whelan et al. [76] is seen to overestimate the experimental results, and the prediction by CFD slightly underestimates the experimental results for the blockage ratios compared.

It is seen that for the range of blockage ratios and boundary conditions considered in this study, the modified form of the blockage model used by Bahaj et al. [1] performs the best in predicting the velocity ratio generated by the current CFD model. There is also good agreement between the current CFD model and experiments for the range of blockage ratios and thrust coefficients compared. However, it is seen in Chapter 6 that many factors influenced the performance of the turbine, and that these factors are not considered in analytical models. Hence, the analytical blockage models should only be used as an estimate to predict the performance of a tidal turbine under blocked conditions.

The actuator disk theory was used in the development of the analytical blockage prediction models used in this section, as well as in the calculation of the velocity ratio that is the metric used for comparison between the CFD results and the analytical models. However, the empirical models used to correct the thrust coefficient of the turbine at high axial induction factors might be able to produce results that is closer to that observed from CFD results, as mentioned in the previous chapter. Thus, the models to correct the thrust coefficient at high axial induction factors can be incorporated into the present models for more robustness in future works.

\subsubsection{Wake prediction}

The ability of the blockage correction models to aid in the prediction of wakes using wake models is also tested. Figure 7.8 shows the wakes predicted by the models presented in section 7.2 .2 compared with the wake predicted by CFD for the low inlet turbulence case at a thrust coefficient of 1 . Required velocity inputs to the wake models are taken from the modified form of the 
blockage correction model used by Bahaj et al. [1].

It is seen that the predictions by both the models have satisfactory agreement with the wake predicted by CFD across the blockage ratios tested. The difference between the two models are seen to be the greatest at low blockage ratios, while they are almost identical at the higher blockage ratios. This is because of the difference in velocities assumed at the turbine location by both models. It is also seen that $k$ for the Jensen model is constant for the range of blockage ratios tested, which is a little surprising as the wake recovery rates for the different blockage ratios predicted by CFD are different. Nevertheless, the constant $k$ value (in equation 7.9) across the different blockage ratios for the Jensen model is consistent with the conclusion that $k$ is mainly affected by the turbulence intensity of the flow. Hence, once the Jensen model has been calibrated for use with a certain hydrodynamic condition, it does not have to be re-calibrated if the environment were to change. However, the $k *$ values (in equation 7.13) for the model by Bastankhah and Porté-Agel [9] increases with increasing blockage ratio. This makes it less user friendly as the model needs to be re-calibrated every time the environment changes.

\subsection{Concluding remarks}

A study has been performed to determine the accuracy of various analytical blockage correction models and wake prediction models seen and used by researchers by comparing them with CFD simulations. The blockage correction models by Bahaj et al., Whelan et al. and Garrett and Cummins were compared against CFD simulations and experimental data. The performance prediction by the current CFD model is seen to provide a good match with experimental results, and the model by Bahaj et al. is found to provide the best agreement against the results obtained by CFD. Higher thrust experienced in CFD simulations compared to that predicted by analytical models at lower blockage ratios have been found to be the result of local acceleration of the flow around the turbines, while enhanced turbulent wake mixing at higher blockage ratios caused the thrust levels experienced 


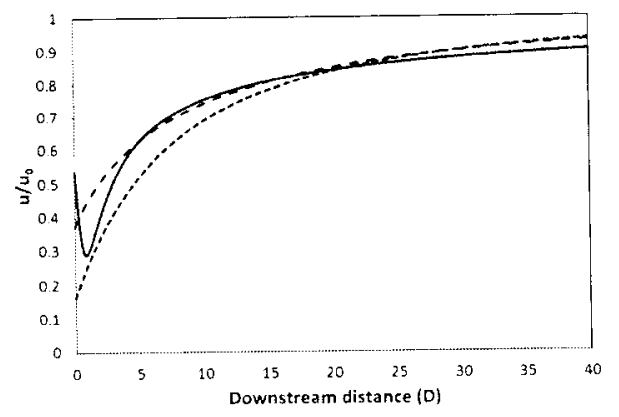

-Channelo --.Jensen $k=0.06--$ Gaussian $k^{*}=0.015$

(a)

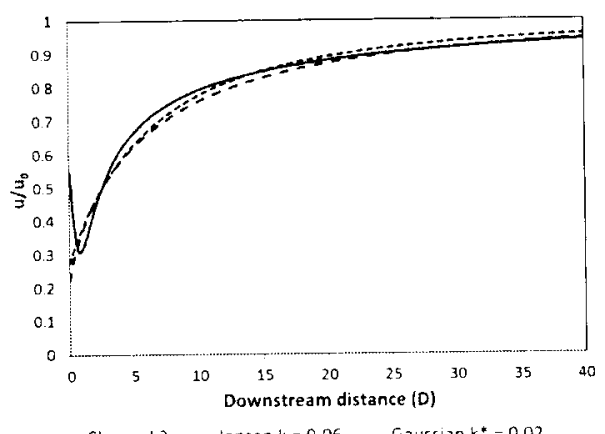

(c)

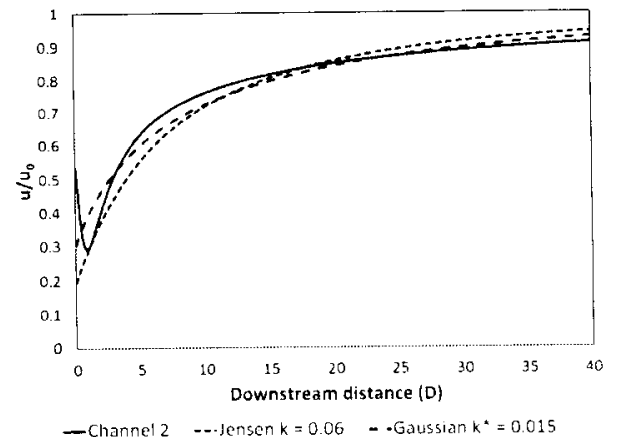

(b)

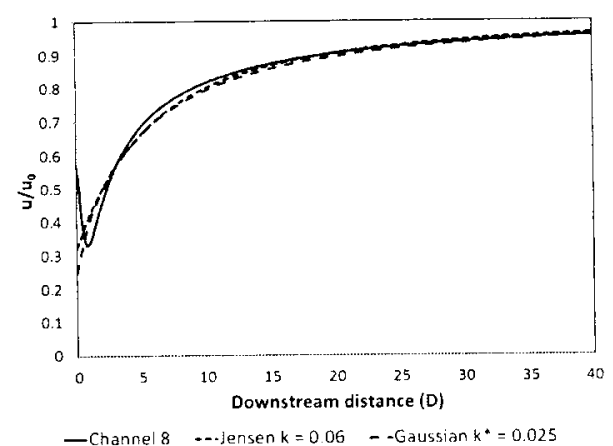

(d)

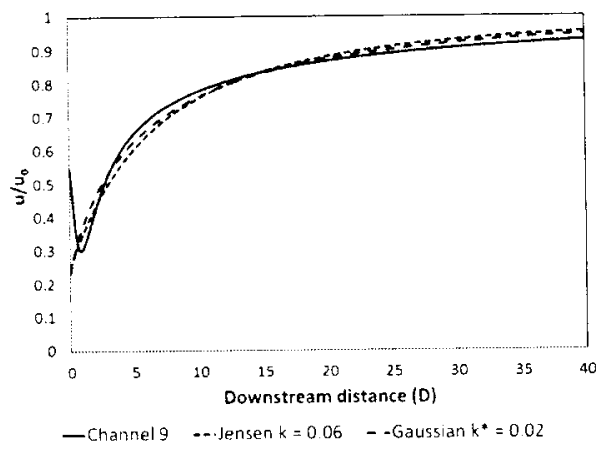

(e)

Figure 7.8: Wake recovery for the different channels predicted with $\mathrm{CFD}$ compared with the modified Jensen wake model and the modified wake model of Bastankhah and Porté-Agel [9] 
in CFD simulations to be lower than that predicted by analytical models.

Increasing blockage ratios have been found to accelerate wake recovery of the turbine in the far-field, while the near-wake recovery is minimally affected for the geometries simulated. The wake predicted by CFD in the different channels were next compared with two analytical wake models. The wake models were originally derived while assuming free-stream conditions have been modified for use in blocked conditions, with the required velocity inputs obtained from the blockage correction models. The modified wake models have been shown to work well in predicting the wakes predicted by CFD across the range of blockage ratios tested. Although the Jensen model is less accurate compared to the wake model with the Gaussian distribution at lower blockage ratios, it's usage is preferred as it can be used with reasonable accuracy across a wide range of blockage ratios without recalibration assuming constant hydrodynamic conditions. Thus, the blockage correction model used by Bahaj et al. paired with the blockage modified Jensen wake model can be a very versatile tool for predicting the performance and wake of a single turbine under various blocked conditions. 


\section{Chapter 8}

\section{Modelling and prediction of the effect of block- age on the performance of a 3 bladed rotor}

This chapter attempts to predict the performance of a 3 bladed rotor by combining the BEM model studied in Chapter 5 and the blockage model studied in Chapter 7. The performance predicted by the chosen combination of models matched well with that predicted by CFD simulations of the same rotor. In addition, modeling the effects of increased Reynolds number experienced by the turbine blades was found to be important in accurately predicting the performance of the turbine. 


\subsection{Introduction}

Blockage studies using the actuator disk model were performed in Chapters 6 and 7 . Although the actuator disk model was used due to its simplicity; it leaves out some details that might be of importance to tidal turbine designers and operators. As such, higher order models need to be considered.

The BEM model has been a reliable analytical model to predict the performance of a turbine given the geometry details of the blade. However, the models which it is based on were derived using free-stream conditions, making it unable to predict performance of a turbine under blocked conditions on its own. Whelan et al. [76] attempted to incorporate their blockage correction model into a BEM code and managed to achieve good agreement with experimental results.

This chapter attempts to integrate the BEM model previously studied in Chapter 5 with the blockage prediction model by Bahaj et al. studied in Chapter 7 to predict the performance of a rotor under blocked conditions. The predictions will also be compared against CFD predictions of the same rotor.

\subsection{CFD model}

The turbine used by Mycek et al. [2] for their water tunnel experiments was chosen as the basis for the CFD model. The turbine is 3 bladed and has a diameter of $0.7 \mathrm{~m}$. The entire turbine geometry except the support structure was resolved using structured mesh generated by ICEM CFD. The turbine was placed mid-depth in a domain with a width of $4 \mathrm{~m}$ and a depth of $2 \mathrm{~m}$, similar to the cross-sectional dimensions of the water tunnel. This results in a blockage ratio of approximately $5 \%$. The velocity inlet was placed 20 turbine diameters upstream of the turbine plane, and the pressure outlet 40 turbine diameters downstream of the turbine plane. A cylindrical inner domain was created around the turbine and the multiple reference frame 
Table 8.1: Size of the different meshes and results for the convergence test

\begin{tabular}{|c|c|c|c|}
\hline & Mesh size & $\mathrm{Cp}$ & $\mathrm{Ct}$ \\
\hline Coarse & 1639338 & 0.412 & 0.711 \\
\hline Medium & 3699857 & 0.434 & 0.740 \\
\hline Fine & 8356575 & 0.437 & 0.742 \\
\hline GCI (\%) & & 2.24 & 0.89 \\
\hline
\end{tabular}

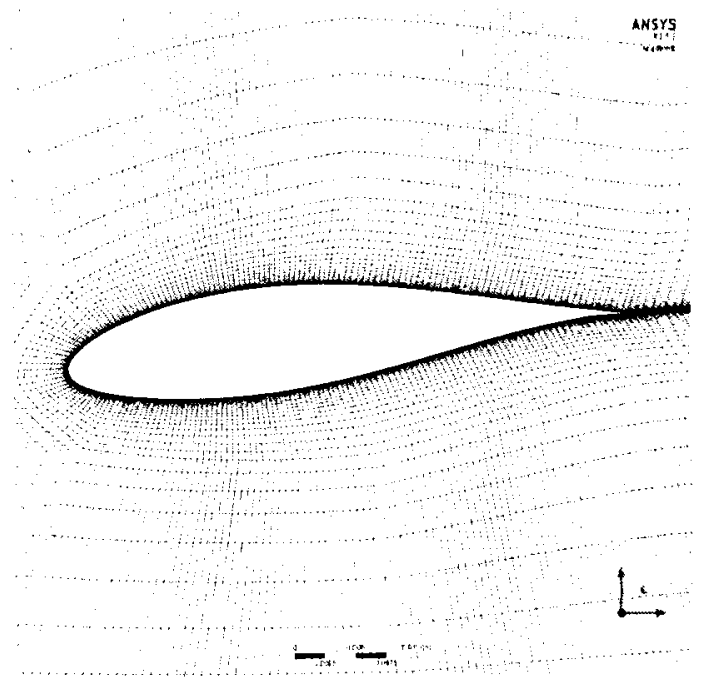

Figure 8.1: Cross section of the mesh around a turbine blade from Mycek et al. [2]

method was used to model the rotation of the turbine.

Three levels of meshes were created and the convergence was measured using the Grid Convergence Index (GCI) [107]. The height of the first cell above the turbine blades is such that the measured $y+$ for the blades is less than 5 at the conditions of the convergence test. A flow velocity of $1.2 \mathrm{~m} / \mathrm{s}$ was prescribed for the convergence test, with a inlet turbulence intensity of $2.8 \%$ and a length scale of $1 / 3$ of the channel depth. All lateral and vertical boundaries were given the symmetry condition to remove the effects of the boundary layer. Results were obtained for the turbine operating with a tip speed ratio (TSR) of 4 . Table 8.1 lists the sizes of the different meshes, as well as results of the convergence test. A cross-section of the mesh around the blade is shown in figure 8.1. 


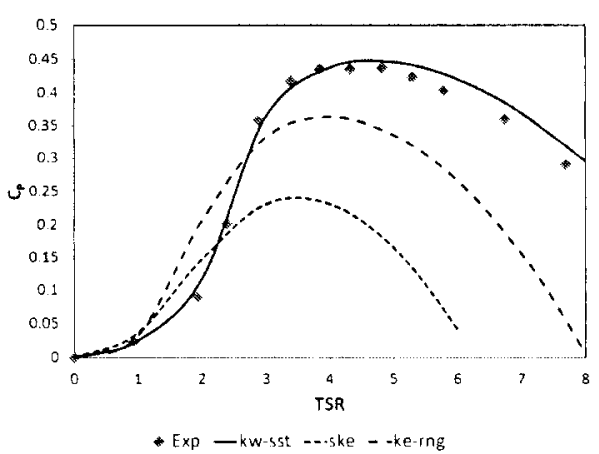

(a)

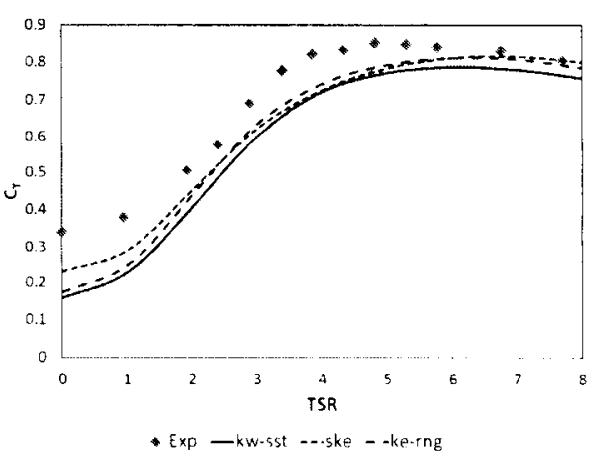

(b)

Figure 8.2: Validation of the CFD model with results obtained with different turbulence models

\subsection{Validation}

The model was tested with three commonly used RANS turbulence models, and the results are presented in figure 8.2. Only RANS models were considered as they offer the best balance between accuracy and computational requirements. No mention of any blockage correction being made was found in [2], thus the presented results is assumed to be as measured from the experiments. As such, no blockage correction is applied on the results produced by CFD as well.

It is seen that the $k-w$ SST turbulence model matches the experimental power coefficient the best, as similarly observed by other authors [61]. Difference in thrust coefficient is due the support structure not being modelled, whereas drag from the whole structure was measured during the experiments. As such, the $k-w$ SST turbulence model was used in all subsequent simulations. 
Table 8.2: Details of the channels used to study the blockage effect

\begin{tabular}{|c|c|c|c|}
\hline Channel & Width $(\mathrm{m})$ & Depth $(\mathrm{m})$ & Blockage ratio (\%) \\
\hline B0 & 4.0 & 2.0 & 4.81 \\
\hline B1 & 2.0 & 2.0 & 9.62 \\
\hline B2 & 1.4 & 2.0 & 13.74 \\
\hline
\end{tabular}

\subsection{Methodology}

Two smaller channels were created by narrowing the channel, with the dimensions of all the channels and the resulting blockage ratios being presented in table 8.2. Channel B0 represents the original channel used in the experimental studies, and channel B2 represents the case where the turbine is in an infinite array with a lateral spacing of 2 turbine diameters.

Inlet conditions used for the convergence test was prescribed for all the different channels. Data was collected for the turbine operating at the same rotational speeds for all channels.

BEM and the blockage prediction model by Bahaj et al. were used to predict the performance of the turbine operating in the different channels. The BEM model used is the same as that used in Chapter 5, where the lift and drag coefficients of the different blade elements are dependent on the Reynolds number of that element. The BEM model was first run with the same rotational speeds as the CFD simulations. The thrust coefficients calculated at each rotational speed were than used as input to the blockage prediction model to predict the velocity that is experienced by the turbine for that particular rotational speed. The BEM model is then run again with the same rotational speed as before, but now with the experienced velocity as the free-stream velocity instead of the velocity in the channel. The new power and thrust coefficients obtained from the experienced velocity was then multiplied by the velocity ratio to calculate the blocked power and thrust coefficients. This process is visually described in figure 8.3.

The blocked power and thrust coefficients calculated from BEM were than 


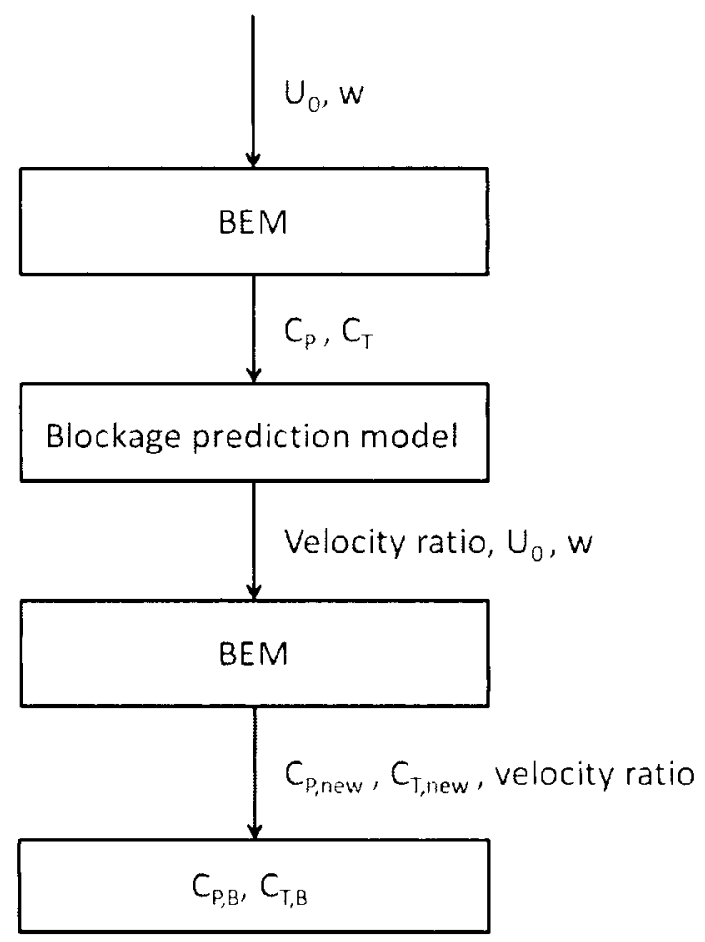

Figure 8.3: Flowchart illustrating the process to predict the blocked performance of a turbine using BEM and a blockage prediction model 


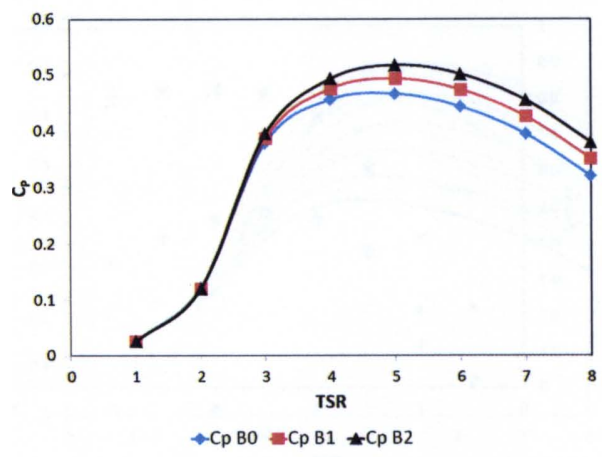

(a)

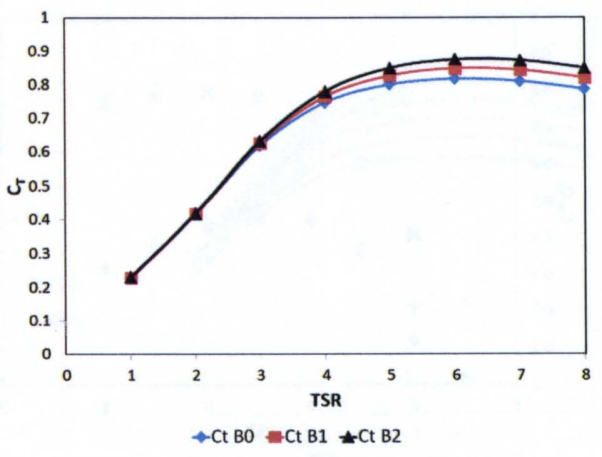

(b)

Figure 8.4: a) Power and b) Thrust coefficients predicted by CFD in the different channels

compared with that predicted from CFD.

\subsection{Results}

\subsubsection{CFD modeling}

Figure 8.4 shows the power and thrust coefficients for the different channels.

It is seen in figure 8.4 that there is a noticeable increase in the power and thrust coefficients as the blockage ratio increased. This runs contrary to that observed by Kolekar and Banerjee [68] in figure 7, which showed that the power coefficient curve remained roughly constant for blockage ratios up till $11 \%$ for the range of TSR tested. The difference in behaviour can be attributed to the boundary walls being given the no-slip condition in the simulations performed by Kolekar and Banerjee [68], thus allowing the boundary layer to develop. Thus, any increase in the power coefficient due to blockage effects is off-set by an increase in power drop due to the boundary layer effect as was illustrated in Chapter 6. 


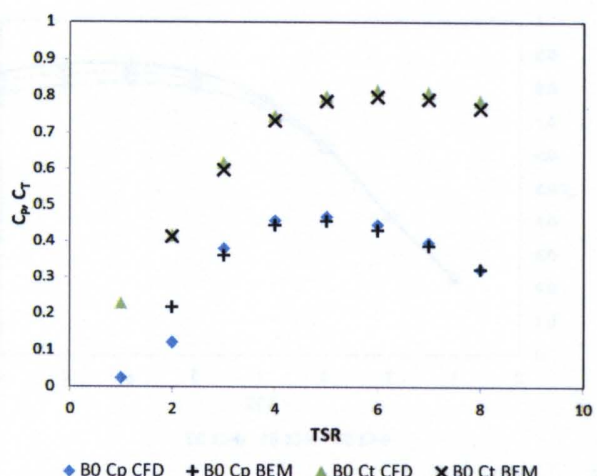

(a)

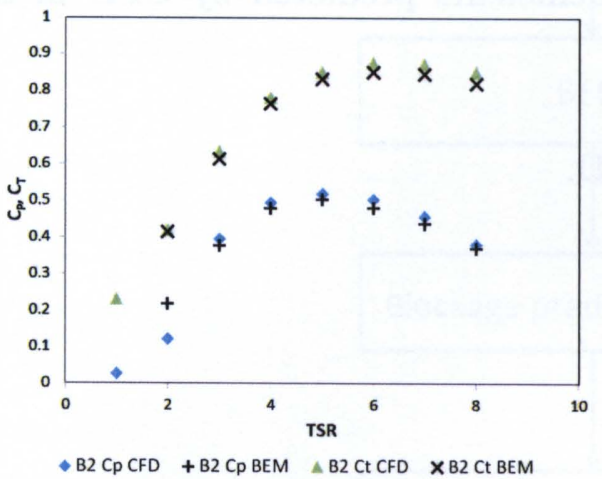

(c)

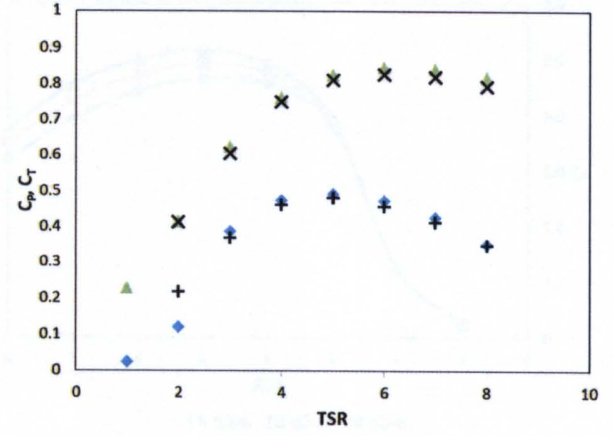

- B1 CPCFD + B1 Cp BEM $\triangle$ B1 Ct CFD X B1 Ct BEM

(b)

Figure 8.5: Comparison between the power and thrust coefficients predicted by CFD and BEM for a) channel B0, b) channel B1, c) channel B2

\subsubsection{BEM prediction}

Figure 8.5 presents the comparison between the power and thrust coefficients predicted by CFD with that predicted by BEM. It is seen that the current combination of BEM and blockage prediction model is able to provide a good prediction of the blocked performance of the rotor over most of the rotational speeds for the blockage ratios considered.

The current prediction method also reveals the mechanism for the increase in performance coefficients of the turbine due to blockage. The increase in power and thrust coefficients can be attributed to two factors. The first factor is the increase in velocity that is experienced by the turbine blades, 


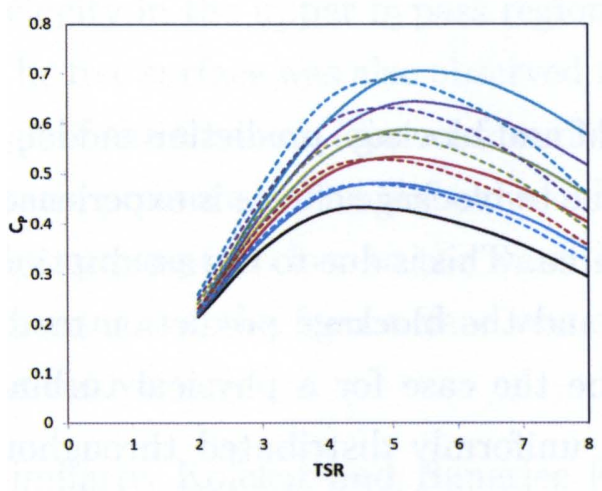

$$
\begin{aligned}
& -B=0 \quad-C p w / R E, B=0.1-C p w / R E, B=0.2 \\
& -C p w / R E, B=0.3-C p w / R E, B=0.4-C p w / R E, B=0.5 \\
& --C p w / o \text { RE, } B=0.1-C p w / o \text { RE, } B=0.2-C p w / o \text { RE, } B=0.3 \\
& --C p w / o \text { RE, } B=0.4-C p w / o \text { RE, } B=0.5
\end{aligned}
$$

(a)

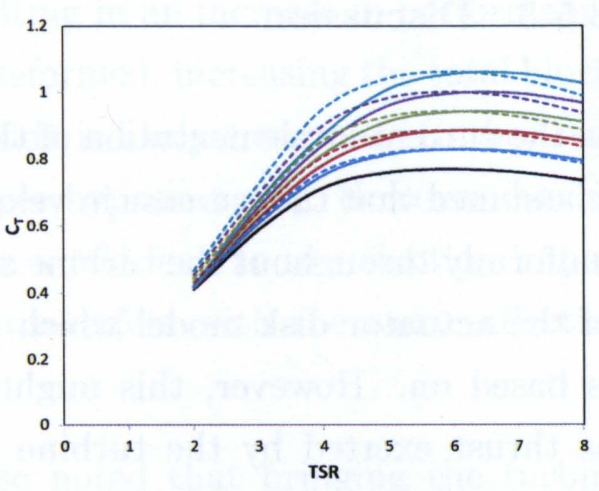

$-B=0 \quad-C t w / R E, B=0.1-C t w / R E, B=0.2$

$-\mathrm{Ct} w / \mathrm{RE}, \mathrm{B}=0.3-\mathrm{Ct} w / \mathrm{RE}, \mathrm{B}=0.4-\mathrm{Ct} w / \mathrm{RE}, \mathrm{B}=0.5$

$\cdots C t w / o$ RE, $B=0.1-\cdots$ Ct $w / o$ RE, $B=0.2 \cdots C t w / o R E, B=0.3$ -..Ct $w / o$ RE, $B=0.4-\cdots C t$ w/o RE, $B=0.5$

(b)

Figure 8.6: Comparison between the a) power coefficients and b) thrust coefficients with (solid lines) and without (dashed lines) the Reynolds number effect

and the second factor is the increase in lift and drag coefficients as a result of the increased Reynolds number over the blade. Both factors must be modelled in order to accurately predict the performance of a turbine in blocked conditions. The Reynolds number effect is also discovered to be the mechanism that caused the increase in optimum rotation speed as the blockage ratio increases, as observed by a few authors $[68,76]$. This is illustrated in figure 8.6, which provides a comparison of the prediction of the performance of the turbine for different blockage ratios with and without the Reynolds number effect.

It is also seen in figure 8.6 that the maximum power and thrust coefficients are similar whether the Reynolds number effect is considered or not. Thus, it might not be necessary to consider the Reynolds number effect if just a rough gauge of the maximum power and thrust coefficient is needed. 


\subsubsection{Discussion}

In the current implementation of the BEM and blockage prediction model, it is assumed that the increase in velocity due to blockage effects is experienced uniformly throughout the turbine swept area. This is due to the assumptions of the actuator disk model which BEM and the blockage prediction model is based on. However, this might not be the case for a physical turbine, as thrust exerted by the turbine is not uniformly distributed throughout the turbine swept area. Although this might not affect the total predicted power and thrust, the power and thrust distribution along the blades might be affected.

The study has important implications for the accuracy of the correction of blocked coefficients obtained in experiments to obtain the blockage-free coefficients. The presence of the boundary layer in water tunnels means that the measured coefficients would be lower than the case where the turbine sees a uniform flow. The coefficients are corrected after obtaining the blockagefree velocity. However, the fact that the lift and drag of the turbine blades are affected by the change in velocity is often overlooked. This effect would be significant when the blockage ratio during experiments are high, meaning that the difference between the blockage-free velocities and tunnel velocities are large. This effect would be rather difficult to account for without the use of models, which would also be subjected to their own uncertainties.

For cases where the blockage ratio is small, the difference between the blockage-free velocity and the tunnel velocity would likely be small. Effect of the change in velocity on the lift and drag of the turbine blades would than also be small and is likely able to be ignored.

Simulations and predictions performed so far have ignored the effects that happen when the turbine is brought close to the fluid boundaries. For cases where the tip of the turbine is closer to the fluid surface, the free-surface effect would likely become significant. Kolekar and Banerjee [68] noted that having the turbine closer to the water surface lead to an increase in the fluid 
velocity in the upper bypass region, resulting in an increase in performance. The free surface was also observed to be deformed, increasing the total blockage of the channel. The surface deformation is likely a response to the lower static pressure in the higher velocity upper bypass region. However, having the turbine too close to the water surface would lead to to reduction in performance as the free-surface was seen to interfere with the wake, affecting its recovery.

Similarly, Kolekar and Banerjee [68] also noted that bringing the turbine close to the bed affected the turbine's performance, and Keogh et al. [117], Myers and Bahaj [97] showed that the wake recovery suffered when the turbine was brought close to the channel boundaries. Although the thrust experienced by the turbine was not reported by Myers and Bahaj [97], Keogh et al. [117], it is likely that the thrust levels had also been reduced due to less flow passing through the disk.

\subsection{Concluding remarks}

This chapter presents a study to predict the performance of a turbine in blocked conditions using BEM and a blockage prediction model. The power and thrust predictions obtained by BEM together with the blockage prediction model had good agreement when compared with predictions obtained using CFD for most of the rotation speeds. Through the use of BEM, it was discovered that the increase in turbine performance due to blockage was due to the increase in velocity experienced by the turbine, as well as an increase in lift and drag brought about by the increase in Reynolds number over the blades. This change in lift and drag of the turbine blades also slightly modified the turbine characteristics, causing the increase of the optimum rotation speed of the turbine. 


\section{Chapter 9}

\section{Performance and wake recovery of a tidal tur- bine array under various blockage conditions}

This chapter expands on the work in Chapter 6 to an array of 5 turbines. The local and global blockage of the channel were varied and their effect on the performance of the turbine was studied. The performance of the turbines within the array was matched to that of a single turbine operating with a certain blockage ratio. By performing a fit with the CFD results, an expression describing the single turbine blockage in terms of the local and global blockage ratio was obtained. Wake recovery of the array was also studied, and the wake was found to recover quicker as the global blockage ratio increases when comparing between channels with the same depth. If the wakes within the array did not merge, they can be estimated by the wake of a single turbine with the same global blockage ratio. 


\subsection{Introduction}

As tidal turbine technology matures, more turbines are going to be deployed in arrays. There is much research on arrays, with most of them focusing on optimising the spacing between turbines by various methods. However, there are comparatively few works on determining the performance of the turbines within the array. It has been shown that placing a turbine in close proximity to boundaries can enhance its performance. Hence, a turbine within an array would surely have its performance enhanced due to its close proximity to the other turbines in addition to the channel bottom and free-surface. Myers and Bahaj [80] noted in experiments of a pair of actuator disk that the measured thrust coefficients increased if the disks were brought too close together. Based on the work of Garrett and Cummins [5], Nishino et al. $[83,84]$ studied this issue and derived analytical expressions to describe the power and thrust coefficients experienced by an array partially spanning a channel. Vennell $[124,125,126]$ derived a model for an array spanning the whole channel, including the effects of channel dynamics.

Wake recovery of an array is also important in determining the distance between rows. Many studies have been done on the effect of lateral turbine spacing on wake recovery of a row of turbines $[80,56,96]$, which naturally extends to the placement of staggered arrays [56]. However, there are only very few studies on the effect of blockage on the wake development of an array.

This chapter expands the study performed in Chapter 6 of a single turbine by investigating the variation in performance of an array as a result of varying local and global blockages, as well as effects on the wake recovery.

\subsection{Methodology}

The numerical model used in this study is adapted from the model that is described in Chapter 4. The model was expanded to include more turbines 


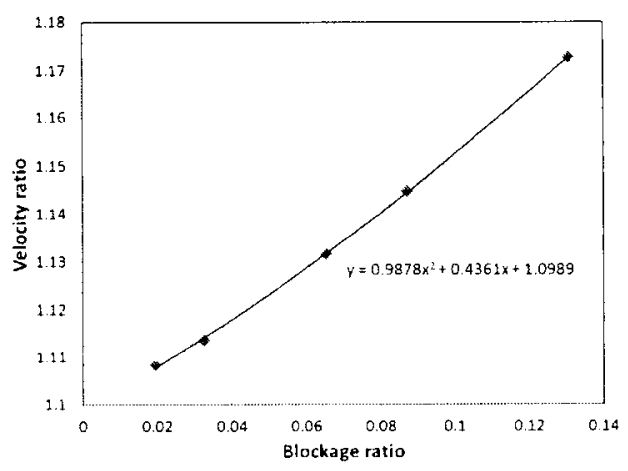

Figure 9.1: Variation of the velocity ratio with changing blockage ratio for a single turbine taken from Chapter 6

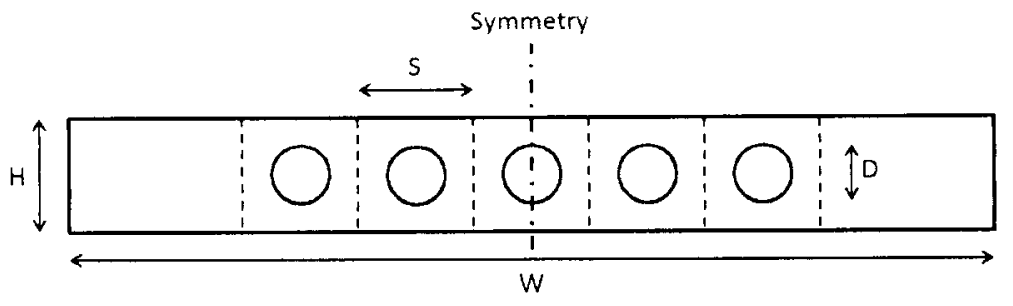

Figure 9.2: Schematic of the array of turbines in a channel

while keeping the mesh densities similar.

Results for simulations performed previously in Chapter 6 with a single turbine is reproduced in Figure 9.1, and will be used as reference for results presented in Section 9.3.

An array of five turbines arranged laterally was placed in channels of varying widths, with inter-turbine spacings of 1.5 to 4 turbine diameters measured from the turbine centres being considered. Depth of the channel was kept constant at 3 turbine diameters. Figure 9.2 shows a schematic of the array within the channel. Half the channel is simulated for computational efficiency, with the dotted-dashed line in the middle representing the symmetry plane. The channel boundaries were assigned the symmetry condition. Thus, the effects of the boundary layer are not considered in this study. A uniform inflow was prescribed, with an inlet velocity of $0.333 \mathrm{~m} / \mathrm{s}$, inlet turbulence intensity of $4.7 \%$ and a turbulence length scale of $0.1 \mathrm{~m}$. 
Table 9.1: Values of the global and local blockage ratios used in CFD simulations

\begin{tabular}{|c|c|c|}
\hline & Global (G) & Local (L) \\
\hline 1 & 0.019 & 0.174 \\
\hline 2 & 0.033 & 0.131 \\
\hline 3 & 0.066 & 0.087 \\
\hline 4 & 0.087 & 0.066 \\
\hline
\end{tabular}

The global, local and array blockage ratios are used to characterise the array, and are defined in equations $9.1,9.2$ and 9.3 respectively.

$$
\begin{gathered}
B_{G}=\frac{N A}{W H} \\
B_{L}=\frac{A}{S H} \\
B_{A}=\frac{B_{G}}{B_{L}}=\frac{N S}{W}
\end{gathered}
$$

where $N$ represents the number of turbines in the array.

Table 9.1 lists the global and local blockage ratios used. This results in 16 combinations of global and local blockage ratios. However, it is physically impossible for the combination G4L4 to happen as the global blockage is higher than the local blockage, which means that the turbines will have to extend beyond the limits of the channel.

Furthermore, 4 other combinations where the local blockage equals the global blockage were considered during the analysis of results, but not simulated. The 4 additional combinations are G1G1, G2G2, L1L1 and L2L2.

The velocity ratio, $\gamma$, defined in equation 4.6 is computed for each turbine and compared across the different arrays. Unless stated otherwise, all results presented are obtained at a thrust coefficient of 1 . 


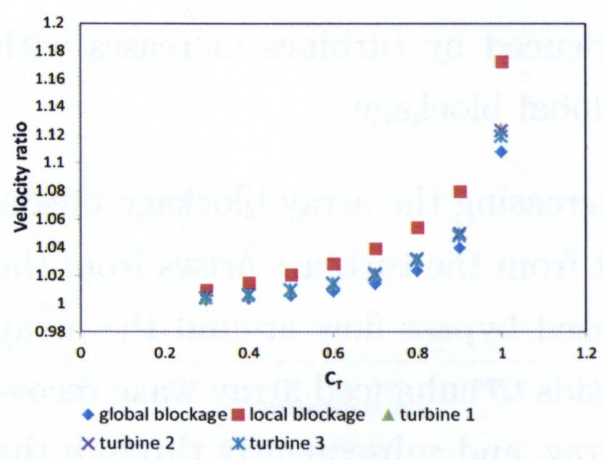

Figure 9.3: Velocity ratio experienced by each turbine in the array compared with that experienced by a single turbine at the same global and local blockage ratio for different thrust coefficients

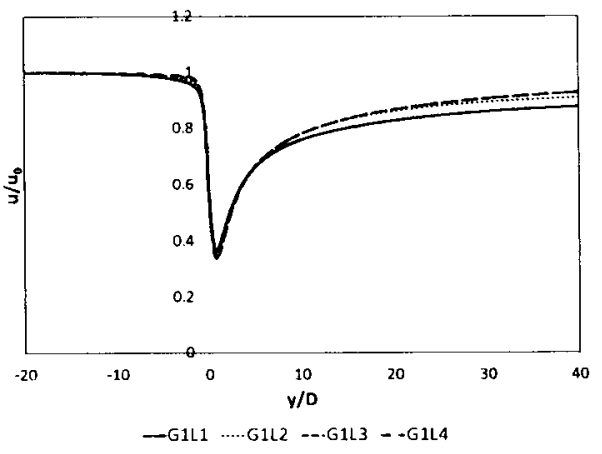

Figure 9.4: Velocity profile along the centreline of the array for various local blockage at global blockage G1

\subsection{Results}

\subsubsection{Array performance}

Figure 9.3 shows that velocity ratio of the turbines within the array is higher than that of a single turbine working in a channel with the same global blockage ratio, and lesser than that of a turbine working in a channel with the same local blockage ratio. This could be due to the fact that free-stream velocity experienced by a turbine in an array is not the same as the freestream velocity experienced by a stand-alone turbine, as the free-stream velocity has been affected by the presence of the array before it reaches the individual turbine. Velocity reduction caused by the presence of the array is seen to decrease with increasing array blockage as seen in figure 9.4 , meaning higher velocities seen by the individual turbines.

For a constant global blockage, increasing array blockage means smaller local blockage. However, the increased velocity seen by the turbines is offset by a weaker local blockage effect. Conversely, the slower velocity seen by the turbine for the case of smaller array blockage is offset by the increased 
local blockage effect. Thus, thrust experienced by turbines increases with increasing local blockage for a constant global blockage.

For the case of constant local blockage, increasing the array blockage means a higher global blockage. Increased thrust from the turbines arises from the blockage effect on the array scale. Increased bypass flow around the array resulting from increased global blockage leads to enhanced array wake recovery. This allows more flow through the array, and subsequently through the turbines as well, leading to increased thrust as array blockage is increased.

It is also seen in figure 9.3 that the velocity ratios of the 3 turbines are almost the same. Thus, the average velocity ratio of the 3 turbines should be a good gauge of the performance of each turbine in the array. The equivalent single turbine blockage, $B_{e q}$, of the turbines within the array can then be calculated from the relationship presented in figure 9.1 using the average velocity ratio. The equivalent single turbine blockage is defined as the blockage ratio required for a single turbine operating in isolation to achieve the same performance of the turbine within an array. Since the performance of the turbines within the array can be predicted using an equivalent single turbine blockage, it is of interest to define a relationship that describes the behaviour of the equivalent blockage as a function of the global and local blockage ratios of the channel. The expression describing the equivalent single turbine blockage is assumed to take the form shown in equation 9.4 .

$$
B_{e q}=f\left(B_{L}\right) B_{G}^{m}+g\left(B_{G}\right) B_{L}^{n}+C
$$

where $C$ is a constant.

Figure 9.5 shows the variation of $B_{e q}$ with respect to the global blockage ratio for each local blockage ratio, while figure 9.6 shows the variation of $B_{e q}$ with respect to the local blockage ratio for each global blockage ratio.

The values of $m$ and $n$ that provide the overall best fit curve for all data 


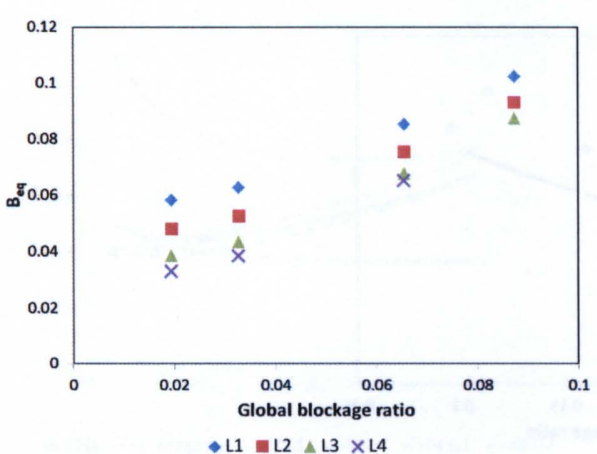

Figure 9.5: Variation of the calculated equivalent single turbine blockage with the global blockage ratio for the different local blockage ratios

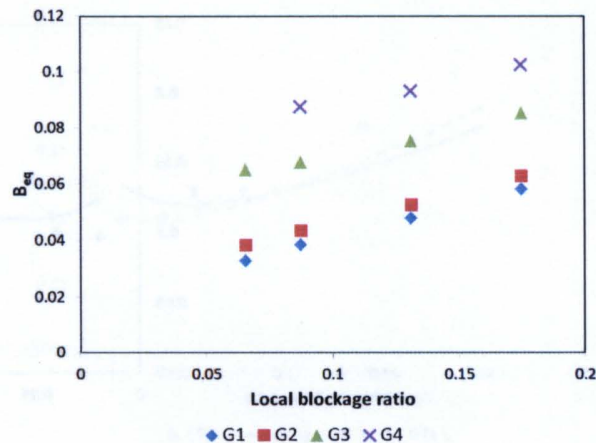

Figure 9.6: Variation of the calculated equivalent single turbine blockage with the local blockage ratio for the different global blockage ratios

shown in figures 9.5 and 9.6 are found to be 1.430 and 1.100 respectively. However, it is to be noted that the values stated do not provide the best fit curve for each data set. It is also seen that the gradients for all the lines in figures 9.5 and 9.6 are different. $f\left(B_{L}\right)$ is thus the equation that describes the gradient of the lines in figure 9.5 , while $g\left(B_{G}\right)$ is the equation that describes the gradient of the lines in figure 9.6. $C$ is obtained by taking the root mean square of the difference between the values obtained by CFD and the values obtained by equation 9.4 after substituting all the relevant blockage values.

Equation 9.4 is then tested by performing additional CFD simulations of an array with a local blockage ratio of 0.4 . This would involve placing a turbine in fluid enclosed by square boundaries with sides of 1.4 diameters. This is physically not advisable as the boundary layer and free-surface would significantly impact the performance of the turbine [68]. The channel width is varied such that the global blockage ratio varies from 0.01 to 0.2 .

Figure 9.7 shows the comparison between results obtained by CFD and equation 9.4. It is seen that the equivalent blockage predicted by equation 9.4 tend to overestimate the CFD results at low global blockage ratios and underestimate the CFD results at higher blockage ratios. Accuracy seems to 


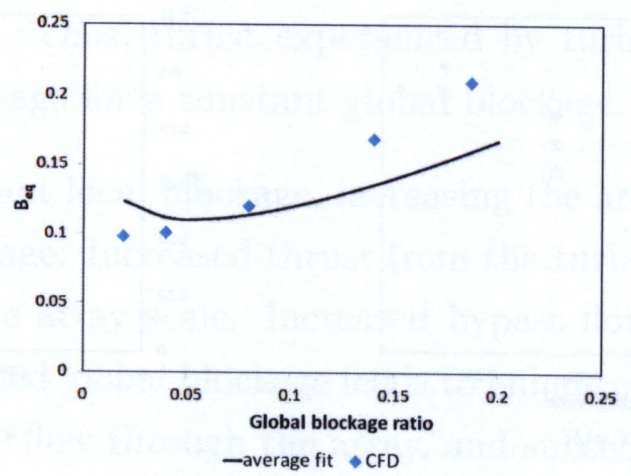

Figure 9.7: Comparison of the predictions obtained by the overall best fit equation with results obtained by CFD simulations for arrays with a local blockage of 0.4

be best for global blockage ratios of 0.05-0.1. Differences between CFD results and that obtained by equation 9.4 could be due to the fact that the exponents in equation 9.4 do not provide the best fit line for all CFD data seen in figures 9.5 and 9.6, but provides the overall best fit line for all the CFD data. Small errors between the fitted equation and CFD data are thus amplified when blockage ratios beyond that used for fitting the equation are used.

$m$ and $n$ are next varied individually to provide the best fit for each of the 2 extreme data sets in figures 9.5 and 9.6. They are L1 and L4 for figure 9.5, and G1 and G4 for figure 9.6. Figure 9.8 shows the results of the alternative fittings compared with the fitting shown in figure 9.7 .

It is seen that the fitting for the L1 data set in figure 9.5 improves the prediction at higher global blockage ratios, while the fitting for G1 data set in figure 9.6 improves the prediction at lower global blockage ratios. Combining results from the two fittings leads to better fitting of the CFD data, which is shown in figure 9.9. Values for $m$ and $n$ for the new fit are found to be 1.300 and 0.780 respectively, and the new expression is given in equation 9.5 . 


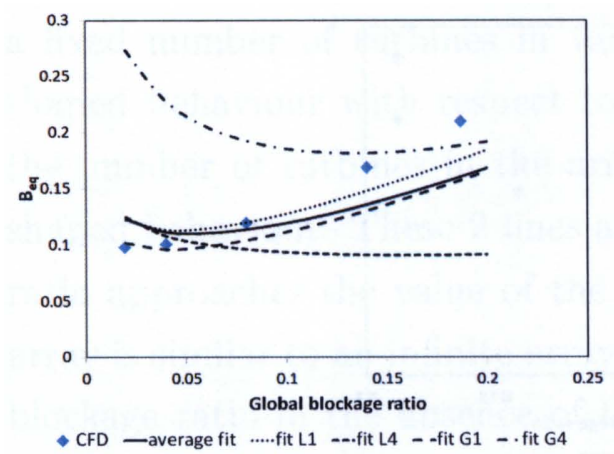

Figure 9.8: Comparisons of predictions obtained by alternative fittings with the original fitting and results obtained from CFD simulations for arrays with local blockage ratio of 0.4

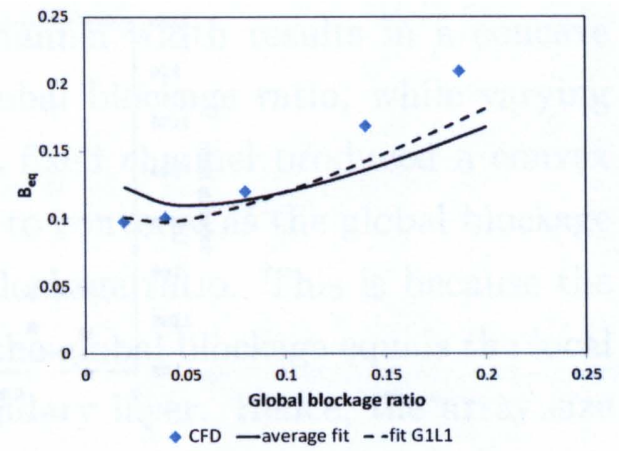

Figure 9.9: Comparison of the new fitting with the original fitting and results obtained from CFD simulations for arrays with local blockage ratio of 0.4

$$
B_{e q}=\left(-1.428 B_{L}^{0.550}+1.751\right) B_{G}^{1.300}+\left(0.004 B_{G}^{-0.700}+0.121\right) B_{L}^{0.780}+0.007
$$

Equation 9.5 is fitted with data obtained from turbines operating at a freestream thrust coefficient of 1 . However, turbines are not expected to achieve that thrust coefficient during normal operations. The accuracy of the model is thus tested at a lower thrust coefficient. CFD simulations of a single turbine operating at a thrust coefficient of 0.7 are performed using the equivalent single turbine blockage predicted by equation 9.5 for the CFD cases shown in figure 9.9. This is compared with simulations of the same CFD cases illustrated in figure 9.9 , with each turbine in the array having a thrust coefficient of 0.7. Results are presented in figure 9.10 .

It is seen that there is good agreement between the two sets of data, and that the behaviour of the predictions seen in figure 9.10 is similar to that seen in figure 9.9. This shows that despite being fitted at a thrust coefficient of 1 , equation 9.5 also works reasonably well when predicting the performance at other thrust coefficients. 


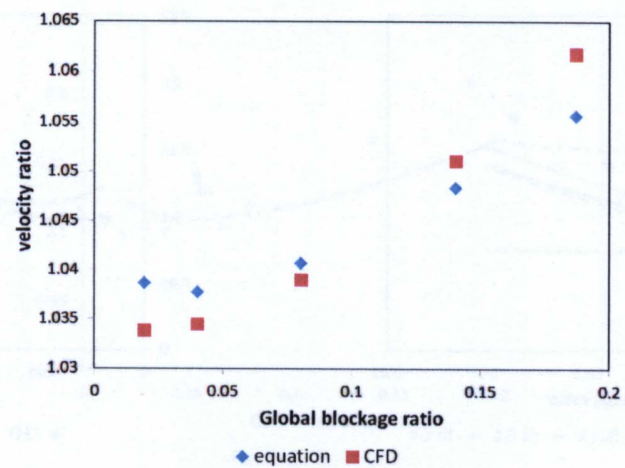

Figure 9.10: Comparisons of the average velocity ratio experienced by each turbine between that predicted using the equivalent single turbine blockage obtained from equation 9.5 and that obtained by CFD simulations for an array operating at a thrust coefficient of 0.7

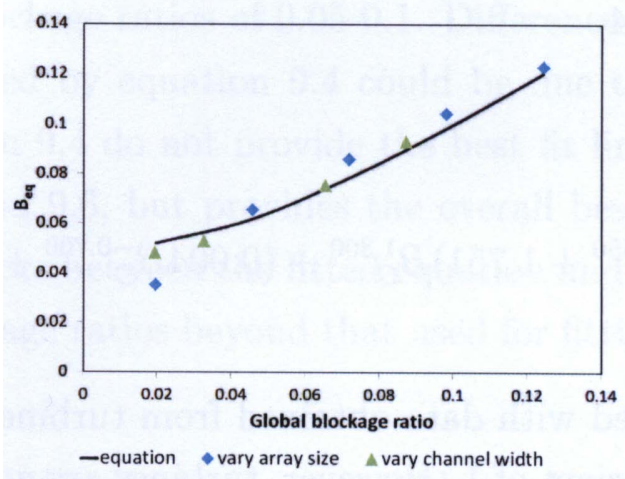

Figure 9.11: Comparisons between the equivalent single turbine blockage obtained by the different methods of varying the global blockage ratio and that predicted by equation 9.5

CFD simulations used to fit equation 9.5 had a fixed array size of 5 turbines. The equation is next tested with arrays with varying number of turbines in a fixed channel, where turbines are added till the array spans the whole channel. Thus, the global blockage ratio is varied by a different method. Local blockage is fixed at L2 in table 9.1.

Figure 9.11 shows the comparison of the equivalent single turbine blockage obtained by different methods, as well as that predicted by equation 9.5 . It is seen that the two methods of varying the global blockage ratio produced different behaviours in the equivalent single turbine blockage. Having 
a fixed number of turbines in varying channel width results in a concave shaped behaviour with respect to the global blockage ratio, while varying the number of turbines in the array in a fixed channel produced a convex shaped behaviour. These 2 lines are seen to converge as the global blockage ratio approaches the value of the local blockage ratio. This is because the array is similar to an infinite array when the global blockage equals the local blockage ratio in the absence of the boundary layer. Hence, the array size should have no effect on the array performance at this point.

\subsubsection{Wake study}

The effect of different local and global blockages on the wake recovery of the array along the centreline is also studied. Figure 9.4 shows the centreline wake velocity for the different local blockage ratios at the global blockage ratio G1 in table 9.1. It is seen that having too large a local blockage can have a significant effect on the wake recovery of the array, as seen by the difference between recovery rates for lines $\mathrm{L} 1$ and $\mathrm{L} 2$. This is due to the fact that the wakes had merged for configuration L1 and L2, leading to reduced wake recovery. Recovery rates for L3 and L4 are seen to be similar as the turbines are sufficiently separated such that the wakes do not merge. This is in line with the observations found in $[80,96]$. As such, the inter-turbine spacing might be more important in determining wake recovery than the resulting local blockage.

It is also seen in figure 9.4 that wake recovery along the centreline for all lateral spacings is almost identical up to approximately 5 diameters downstream of the array, which lies within the near-wake region. This is despite the fact that flow in the bypass region between turbines are different for the different lateral spacings. This might indicate that what happens in the bypass region between turbines for the length of the near wake do not affect the wake recovery in that region.

Figure 9.12 presents the centreline wake recovery for the different global 


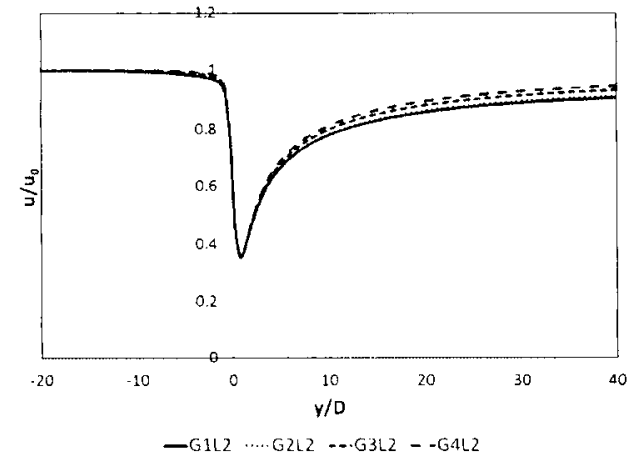

Figure 9.12: Velocity profile along the centreline of the array for various global blockage at local blockage L2

blockage ratios for local blockage ratio L2, while figure 9.13 shows the velocity at 20 diameters downstream for the same cases. It is noticed that the rate of wake recovery for the array increases with increasing global blockage ratios. It is also seen that the wake recovers slightly faster at the edges of the array due to accelerated flow in the bypass region. Increased rate of wake recovery as a result of increased global blockage could be due to the fact that more flow is forced through the array as blockage increases. Increased bypass velocities within the array also implies that more turbulence is generated along the edges of the wake, leading to enhanced wake recovery. This can be seen in figure 9.14, which shows higher levels of turbulent kinetic energy in the wake at 20 diameters downstream as global blockage increases.

This behaviour is also seen for the case of varying array size in a fixed channel, as shown in figure 9.15 .

Figure 9.16 displays the wake recovery for the arrays G1L2 and G1L3 compared with the wake predicted for a single turbine operating at their respective global and local blockage ratios. It is seen that the centreline wake profile for both arrays matches well with that of a single turbine operating at their global blockage ratios. This suggests that where there is no significant merging of wakes within the array, it is possible to have a good approximation of the wake recovery of an array by using the wake of a single turbine operating at the array's global blockage ratio. 


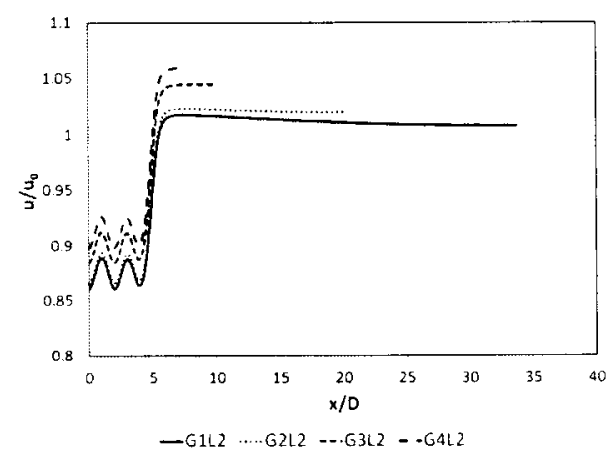

Figure 9.13: Lateral velocity profile for various global blockage ratios for the local blockage L2 taken at $20 \mathrm{D}$ downstream of the turbine plane

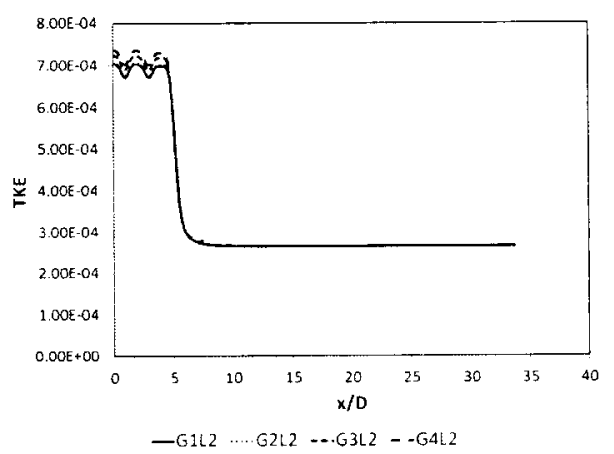

Figure 9.14: Turbulent kinetic energy profile for various global blockage ratios for the local blockage $\mathrm{L} 2$ taken at $20 \mathrm{D}$ downstream of the turbine plane

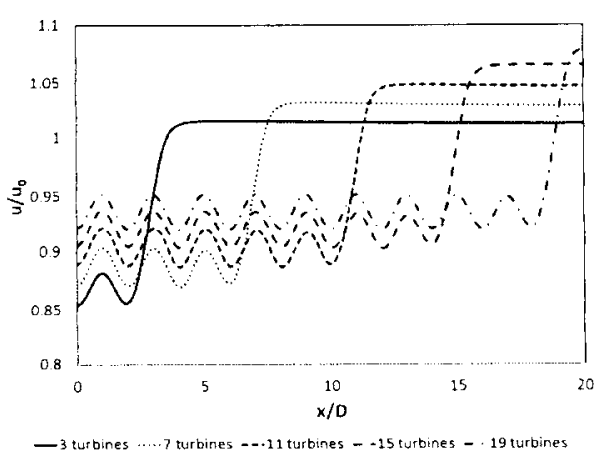

(a)

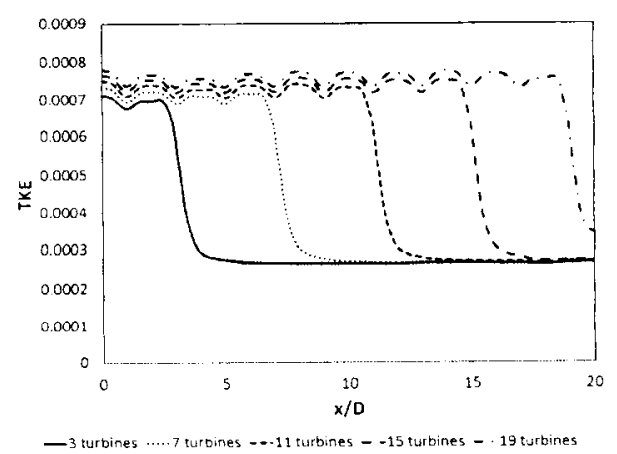

(b)

Figure 9.15: a) Lateral velocity profile and b) turbulent kinetic energy for arrays of various sizes for the local blockage L2 taken at 20D downstream of the turbine plane 


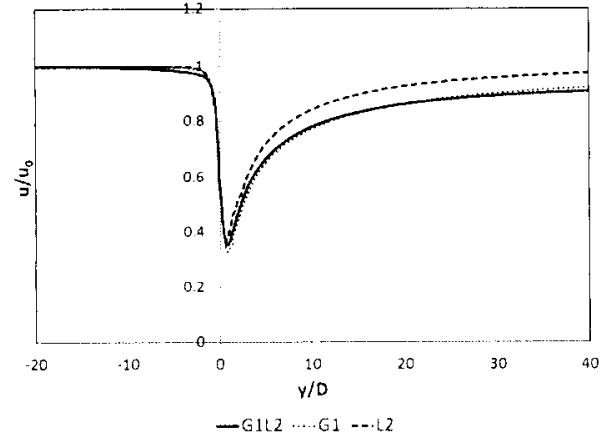

(a)

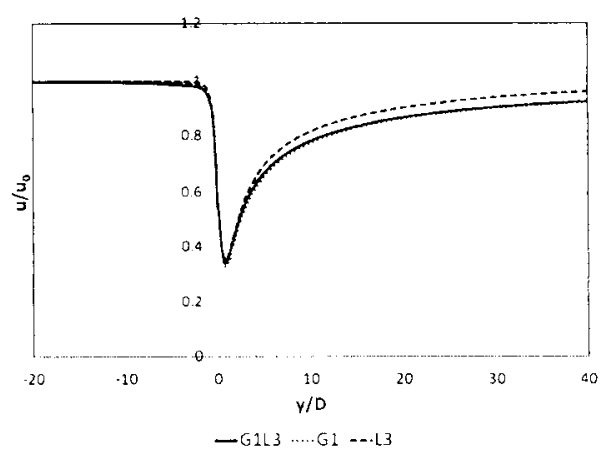

(b)

Figure 9.16: Comparison between the centreline wake recovery of the arrays a) G1L2, b) G1L3 with the wake of a single turbine at their respective global and local blockage ratio

This finding is further supported by figure 9.17 , which compares the centreline wake recovery at $20 \mathrm{D}$ downstream of the central turbine for the different methods for varying the global blockage ratio. The two array cases had the local blockage ratio $\mathrm{L} 2$, while the single turbine result is taken from figure 6.18c. Good agreement is seen between the different methods of varying the global blockage ratio, which means that the finding that wake recovery increases linearly with blockage ratio for single turbines also generally applies to arrays provided that the cases compared had the same channel depth. This makes it possible for single turbine wake prediction models to be used to predict the wake of an array. However, this is only observed for the cases currently considered, where the channel depth is the same for all cases that are compared. More studies are needed to determine the extent of this observation.

\subsubsection{Discussion}

Figures 9.5 and 9.6 show that increasing both the global and local blockages of a turbine array serves to increase the equivalent blockage experienced by each turbine in the array, thus increasing the power and thrust from each turbines. This is in line with the advice to pack as densely as possible a row 


\subsection{Concluding remarks}

A study to investigate the performance of turbines within an array has been performed. CFD simulations of an array of five turbines with various local and global blockage ratios were performed, and it was discovered that performance of all the turbines were almost identical. The concept of the equivalent single turbine blockage was introduced, where the the performance of an array can be represented by a single turbine operating with a particular blockage ratio. An equation predicting the equivalent single turbine blockage of an array was obtained by fitting data obtained from the CFD simulations, and validation of the equation using an array with a local blockage ratio outside of the calibration range showed satisfactory agreement. Although the equation was fitted using data obtained at a thrust coefficient of 1 , it is shown also to work at other thrust coefficients. Performance of the array is found to be affected by array size for low global blockage ratios, but the dependency on array size is reduced as the global blockage ratio tends towards the local blockage ratio.

Wake recovery of the array is also studied. Increasing the global blockage while keeping the local blockage constant is found to result in faster wake recovery, whereas increasing local blockage while keeping global blockage constant is found to result in slower wake recovery. It is further discovered that recovery of the near wake is minimally affected by variations of lateral spacings between turbines. When there are no significant mixing between the wakes of different turbines within the array, it is also found that wake recovery of the turbines within the array can be represented by that of a single turbine operating at the same global blockage ratio as the array. 


\section{Chapter 10}

\section{Conclusion}

This chapter summarises the findings from the entire thesis. Some major findings include the result that blockage causes the velocity experienced by the turbine to increase. Including the effect of the boundary layer causes the predicted performance of the tidal turbine to be reduced. Analytical models to predict the various aspects of tidal turbine operation are also recommended. Potential areas for further work include a more in depth study on the effect of the boundary layer on tidal turbine operation, and further studies on tidal turbine arrays. 


\subsection{Summary}

This thesis presents a series of studies performed to gain a better understanding of the effects of channel blockage on the performance and wake recovery of tidal turbines. Implementation of analytical models for the prediction of the operation of a turbine under blocked conditions is also explored, and guidelines for implementation are given where appropriate.

The accuracy of the BEMT model was first studied as it is the most commonly used analytical turbine model used to predict the performance of a turbine. Effects of the different tip loss functions and the effect of the airfoil Reynolds number were studied as they greatly influenced the output of the model. The Prandtl tip loss function was found to provide the best prediction for most of the cases studied. Allowing the lift and drag coefficient of the airfoil to vary according to the Reynolds number that is experienced increased the power coefficient of the turbine at higher rotational speeds. Prediction at off-design cases also improved compared to using lift and drag coefficient obtained from a single Reynolds number.

A new method of analysing the results from CFD simulations is introduced, using parameters that can be directly measured from the simulations. The fluid velocity that the turbine experienced is calculated, and its ratio to the free-stream velocity is recommended as a metric to determine the effects of blockage on the performance of the turbine.

Using CFD simulations, the effect of blockage on a turbine is found to increase the velocity that is experienced by the turbine without a physical increase in the free-stream velocity. In addition to the amount of blockage, the increase in velocity is also influenced by the free-stream thrust coefficient of the turbine. As such, given the free-stream thrust coefficient of the tidal turbine, the effect of blockage can be quantified by the amount of increase in the experienced velocity over the measured free-stream velocity.

The study of a single turbine under various flow and blockage conditions illustrates the differences between the real world operating environment and 
assumptions made by the analytical models. The main difference being the presence of the boundary layer, which is not accounted for in the analytical models. The presence of the boundary layer causes a drop in predicted power and thrust compared to prediction in the absence of it due to the difference in the flows around the turbine. The discrepancy in predicted thrust grows as the blockage ratio of the channel increases, as larger parts of the flow experienced by the turbine would be affected by the boundary layer. The same behaviour in thrust is observed as the channel was changed from a shallow and wide channel to a narrow and deep channel due to the lateral boundary layers having more influence than the boundary layer from the channel bottom. Thus, it would be more advisable to install turbines in a shallow and wide channel than a narrow and deep channel. The difference in experienced thrust would mean that there is a natural safety factor built in for turbine designers. However, power removed from the flow by the turbine would also be accordingly overestimated.

For the same case of a single turbine in a shallow and wide channel versus a deep and narrow channel of the same area without boundary layers, the deep and narrow channel was found to result in more thrust being produced by the turbine, possibly due to different decaying rates of turbulence in the flow. Thus, comparing different channels with the same depth is recommended for studying the effect of blockage on performance of a turbine to avoid bringing in unintended flow effects. Studies performed for channels with a certain depth would also not be accurate for channels of another depth, although the overall trends are likely to be similar.

Increasing the blockage ratio of a channel increased the thrust produced by the turbine, as well as hastening the recovery of the wake produced. The increase in wake recovery was also found to be proportional to the increase in blockage ratio of the channel. This means that distance between rows of turbines can be shortened in higher blockage environments while achieving similar levels of power extraction.

The performance of various analytical models to predict the effect of blockage 
on the performance and wake produced was also studied. For the case of thrust produced by a turbine under blocked conditions, predictions by the blockage models was found to be more sensitive to changes in blockage ratios when compared to that predicted by CFD. However, there is generally good agreement between the models and CFD for typical ranges of operating thrust coefficients. The thrust predicted by the blockage model used by Bahaj et al. has the best match with that predicted by CFD, while the Jensen wake model has the best balance between usability and accuracy when predicting the wake produced by a turbine under blocked conditions.

The blockage model by Bahaj et al. was next combined with the BEMT model to predict the performance of a 3 bladed rotor under various blockage conditions. The chosen combination of analytical models provided good match for the power and thrust coefficients when compared with CFD predictions of the same rotor for the blockage ratios considered. It was also established that it is important to take into account the increase in lift and drag coefficients as a result of increased velocity experienced by the turbine in a blocked environment. This has the effect of increasing the optimum rotation speed of the rotor as the blockage ratio increases. Failure to do so would result in the optimum rotation speed being wrongly predicted.

An array of five turbines arranged laterally and partially spanning a channel was studied as well. Local blockage was varied by varying the distance between the turbines within the array, while global blockage was varied by varying the channel width. Channel depth was kept constant for all cases. Thrust for each turbine in the array was found to be higher than the thrust predicted by a single turbine operating with the same global blockage as the array, while lower than a single turbine operating with the same local blockage as the array. This puts those thrust levels as the lower and upper bounds respectively of what to expect within an array.

The thrust experienced by each turbine in the array is also matched to that of a single turbine operating in a certain blockage, which is termed as the equivalent single turbine blockage. An expression relating the equivalent 
single turbine blockage to the local and global blockage of the array was obtained by fitting data from CFD simulations of arrays with various local and global blockage ratios. The global blockage ratio was found to have a greater influence on the equivalent single turbine blockage compared to the local blockage ratio. This indicates that it is better to grow arrays by filling up the channel before shortening the inter-turbine distance to get the most out of them.

This was also supported by studying the wake produced by the array. A higher global blockage ratio caused the array wake to recover quicker, while the wake of the different turbines will merge and take longer to recover if the inter-turbine distance is too short. The wake recovery of the centre turbine in the array was also found to increase linearly with the global blockage ratio, as with the case for a single turbine. If there are no significant merging of wakes between the turbines in the array, the centreline wake produced by the centre turbine of the array is found to match that produced by a single turbine operating with the same global blockage as the array. This allows the use of analytical wake models to predict the wake recovery of the array. From the results in this thesis, the lateral distance between turbines in an array is recommended to be no shorter than 2 turbine diameters measured from the turbine centre to take advantage of this fact.

\subsection{Implications}

Some implications can be drawn from the works in this thesis.

The fact that turbines within a closely packed array experiences blockage effects means that their operational characteristics in the array could potentially be very different from when they are operated in isolation. The peak power coefficient would be higher than if it were isolated, and it would occur at a higher rotational speed. Power curves with respect to velocity would also be different.

This has knock-on effects on the accuracy of resource characterisation. Power 
available for extraction might be underestimated if blockage effects are not taken into account. Number of rows that can fit into a given area might also increase due to the increase in wake recovery. However, this might significantly increase the amount of thrust on the flow, leading to reduced flow through the array.

\subsection{Future works}

In the study of a single turbine in a channel, only one boundary layer profile was considered. The study can be expanded to investigate the effects of different profiles on the performance of the turbine. This can include the boundary layer generated by different types of terrains on the channel bed. A model to correct for the presence of the boundary layer could also be developed.

Another follow up study can be on the effects of different channel shapes on the blockage experienced by the turbine, as only rectangular channels were considered in this thesis.

The validation of the blockage prediction models involved comparing against CFD results obtained from channels with the same depth. More study can be performed with channels of different depth and a greater range of blockage ratios to determine the extent of the accuracy of the models.

The expression to describe the blockage experienced by each turbine in an array is only valid for the case of an array of 5 turbines. However, it has been proven that arrays of different sizes have different performance even if they have the same local and global blockage. Hence, it is of interest to extend the expression to take into account the size of the array.

The wake of the array was studied at a relatively low level of turbulence. More work could be done to investigate the wake produced by the array at levels of turbulence typically found in potential tidal sites. This could result in a different minimum inter-turbine spacing within the array than what is 
recommended in this thesis.

Since the effect of blockage on a single row of turbines was studied, this work can also be extended by studying the effect of blockage on multi-row arrays, both in regular and staggered arrangements. Irregular arrangements of turbines would also be of interest as well. This can be further extended to study the effects of blockage on resource characterisation.

Lastly, the actuator disk method is used for the analysis of the CFD results and the formulation of the analytical blockage prediction models. The use of models that correct the thrust coefficient at high axial induction factors can included in future works. 
Conclusion 


\section{References}

(1] Bahaj, A., Molland, A., Chaplin, J., Batten, W.. Power and thrust measurements of marine current turbines under various hydrodynamic flow conditions in a cavitation tunnel and a towing tank. Renewable Energy 2007;32(3):407-426.

[2] Mycek, P., Gaurier, B., Germain, G., Pinon, G., Rivoalen, E.. Experimental study of the turbulence intensity effects on marine current turbines behaviour. Part I: One single turbine. Renewable Energy 2014;66:729-746.

[3] UK: Scottish Power Completes Tidal Turbine Trials. 2012. URL: http://subseaworldnews.com/2012/06/01/uk-scottish-powercompletes-tidal-turbine-trials/.

44 Batten, W., Bahaj, A., Molland, A., Chaplin, J.. The prediction of the hydrodynamic performance of marine current turbines. Renewable Energy 2008;33(5):1085-1096.

[5] Garrett, C., Cummins, P.. The efficiency of a turbine in a tidal channel. Journal of Fluid Mechanics 2007;588:243-251.

[6] Molland, A.F., Bahaj, A.S., Chaplin, J.R., Batten, W.M.J.. Measurements and predictions of forces, pressures and cavitation on 2-D sections suitable for marine current turbines. Proceedings of the Institution of Mechanical Engineers, Part M: Journal of Engineering for the Maritime Environment 2004;218(2):127-138.

[7] Blackmore, T., Batten, W., Harrison, M., Bahaj, A.. The Sensitivity of Actuator-Disc RANS Simulations to Turbulence Length Scale Assumptions. In: 9th European Wave and Tidal Energy Conference. Southampton; 2011, p. 390-399. 
[8] McTavish, S., Feszty, D., Nitzsche, F.. An experimental and computational assessment of blockage effects on wind turbine wake development. Wind Energy 2014;17(July 2013):1515-1529.

[9] Bastankhah, M., Porté-Agel, F.. A new analytical model for windturbine wakes. Renewable Energy 2014;70:116-123.

[10] MeyGen Limited, MeyGen - The tide of change in Caithness. 2013. URL: http://www. meygen.com/.

[11] Burton, T., Jenkins, N., Sharpe, D., Bossanyi, E.. Wind Energy Handbook. Chichester: Wiley; 2011.

[12] Hansen, M.. Aerodynamics of wind turbines. 2nd editio ed.; London: Earthscan; 2008.

13] Batten, W., Bahaj, A., Molland, A., Chaplin, J.. Experimentally validated numerical method for the hydrodynamic design of horizontal axis tidal turbines. Ocean Engineering 2007:34(7):1013-1020.

[14] Bahaj, A., Batten, W., McCann, G.. Experimental verifications of numerical predictions for the hydrodynamic performance of horizontal axis marine current turbines. Renewable Energy 2007;32(15):24792490 .

[15] Himmelskamp, H.. Profile investigations on a rotating airscrew. Tech. Rep.; MAP Volkenrode; 1947.

[16] Lindenburg, C.. Investigation into Rotor Blade Aerodynamics. Ecnnl 2003; (July):114.

[17] Du, Z., Selig, M.. A 3-D stall-delay model for horizontal axis wind turbine performance prediction. In: AIAA-98-0021, 36th AIAA Aerospace Sciences Meeting and Exhibit, 1998 ASME Wind Energy Symposium. Reno, NV: American Institute of Aeronautics and Astronautics; 1998,. 
[18] Mc Croskey, W.J.. Measurements of boundary layer transition, separation and streamline direction on rotating blades. Tech. Rep.; NASA TN-D-6321; 1971.

[19] Eggers, A.J., Digumarthi, R.. Approximate Scaling of Rotational Effects of Mean Aerodynamic Moments and Power Generated by the Combined Experiment Rotor Blades Operating in Deep-Stalled Flow. In: 11th ASME Wind Energy Symposium. Houston, TX; 1992, p. 33-43.

[20| Snel, H., Houwink, R., Bosschers, J.. Sectional prediction of lift coefficients on rotating wind turbine blades in stall. Tech. Rep.; National Aerospace Laboratory; Amsterdam; 1994.

[21] Lindenburg, C.. Modelling of rotational augmentation based on engineering considerations and measurements. In: European Wind Energy Conference. London; 2004, p. 22--25.

[22] Bak, C., Johansen, J., Andersen, P.. Three-dimensional corrections of airfoil characteristics based on pressure distributions. Proceedings of the European Wind Energy Association 2006;:1-10.

[23] Corrigan, J., Schilling, J.. Empirical model for stall delay due to rotation. In: American Helicopter Society Aeromechanics Specialists Conference. San Francisco; 1994,.

[24] Tangler, J.L., Selig, M.. An evaluation of an empirical model for stall delay due to rotation for HAWTS. Tech. Rep.; National Renewable Energy Laboratory; Golden, Co; 1997.

[25] Guntur, S., Bak, C., Sø rensen, N.N.. Analysis of 3D Stall Models for Wind Turbine Blades Using Data from the NEXICO Experiment. In: Proceedings of the 13th International Conference on Wind Engineering. Amsterdam; 2012,.

[26] Yu, G., Shen, X., Zhu, X., Du, Z.. An insight into the separate flow and stall delay for HAWT. Renewable Energy 2011;36(1):69-76. 
[27] Dowler, J.L., Schmitz, S.. A solution-based stall delay model for horizontal-axis wind turbines. Wind Energy 2015;18(August 2014):1793-1813.

[28] Branlard, E.. Wind turbine tip-loss corrections. Masters thesis; Technical University of Denmark; 2011.

[29] Goldstein, S.. On the vortex theory of screw propellers. Proceedings of the Royal Society A: Mathematical, Physical and Engineering Sciences $1929 ; 123(792): 440-465$.

[30] Glauert, H.. Airplane Propellers. In: Durand, W.F., editor. Aerodynamic Theory; chap. XI. Springer Berlin Heidelberg; 1935, p. 169-360.

[31] Wilson, R.E., Lissaman, P.B.S.. Applied aerodynamics of wind power machines. Tech. Rep.; Oregan State University; 1974.

[32] De Vries, O.. Fluid dynamic aspects of wind energy conversion. Tech. Rep.; AGARD-AG-243; 1979.

[33] Shen, W.Z., Mikkelsen, R., Sø rensen, J.N., Bak, C.. Tip loss corrections for wind turbine computations. Wind Energy 2005;8(4):457-475.

[34] Hansen, M.O.L., Johansen, J.. Tip studies using CFD and comparison with tip loss models. Wind Energy 2004;7(April):343-356.

[35] Lee, J.H., Park, S., Kim, D.H., Rhee, S.H., Kim, M.C.. Computational methods for performance analysis of horizontal axis tidal stream turbines. Applied Energy 2012;98:512-523.

[36] Sankar, L.. Computational studies of horizontal axis wind turbines. Tech. Rep.; Georgia Institute of Technology; 2000.

[37] Madsen, H.A., Mikkelsen, R., Ø ye, S., Bak, C., Johansen, J.. A detailed investigation of the Blade Element Momentum (BEM) model based on analytical and numerical results and proposal for modifications of the BEM model. Journal of Physics: Conference Series $2007 ; 75: 012016$. 
|38| Branlard, E., Dixon, K.. An improved tip-loss correction based on vortex code results. In: Proceedings of EWEA 2012 - European Wind Energy Conference \& Exhibition. Copenhagen: European Wind Energy Association (EWEA); 2012,.

[39] Masters, I., Chapman, J.. A robust blade element momentum theory model for tidal stream turbines including tip and hub loss corrections. Journal of Marine Engineering and Technology 2011;10(1):25-36.

[40] Glauert, H.. The analysis of experimental results in the windmill brake and vortex ring states of an airscrew. Tech. Rep.; Aeronautical Research Committee Reports and Memoranda, London: Her Majesty's Stationery Office; 1926.

[41] Buhl Jr, L.. A new empirical relationship between thrust coefficient and induction factor for the turbulent windmill state. Tech. Rep.; NREL; 2005.

[42] Chapman, J., Masters, I., Togneri, M., Orme, J.. The Buhl correction factor applied to high induction conditions for tidal stream turbines. Renewable Energy 2013;60:472-480.

[43] Harrison, M., Batten, W., Myers, L., Bahaj, A.. A comparison between CFD simulations and experiments for predicting the far wake of horizontal axis tidal turbines. IET Renewable Power Generation $2009 ; 4(6): 566-575$.

[44] Batten, W., Harrison, M., Bahaj, A.. Accuracy of the actuator disc-RANS approach for predicting the performance and wake of tidal turbines. Philosophical transactions Series A, Mathematical, physical, and engineering sciences 2013;371:20120293.

[45] Blackmore, T., Batten, W., Bahaj, A.. Influence of turbulence on the wake of a marine current turbine simulator. Proceedings of the Royal Society A: Mathematical, Physical and Engineering Sciences 2014;470(August):20140331-20140331. 
[46] Hunter, W., Nishino, T., Willden, R.. Investigation of tidal turbine array tuning using 3D Reynolds-Averaged Navier-Stokes Simulations. International Journal of Marine Energy 2015;10:39-51.

[47] Bai, L., Spence, R., Dudziak, G.. Investigation of the influence of array arrangement and spacing on tidal energy converter (TEC) performance using a 3-dimensional CFD model. In: 8th European Wave and Tidal Energy Conference. 2009, p. 654-660.

[48] Nishino, T., Willden, R.. Effects of 3-D channel blockage and turbulent wake mixing on the limit of power extraction by tidal turbines. International Journal of Heat and Fluid Flow 2012;37:123-135.

[49] Harrison, M., Batten, W., Bahaj, A.. A blade element actuator disc approach applied to tidal stream turbines. In: Occans $2010 \mathrm{MT}$ S/IEEE Seattle. Seattle: IEEE; 2010, p. 1-8.

[50] Turnock, S.R., Phillips, A.B., Banks, J., Nicholls-Lee, R.. Modelling tidal current turbine wakes using a coupled RANS-BEMT approach as a tool for analysing power capture of arrays of turbines. Ocean Engineering 2011;38(11-12):1300-1307.

[51] Masters, I., Williams, A., Croft, T., Togneri, M., Edmunds, M., Zangiabadi, E., et al. A Comparison of Numerical Modelling Techniques for Tidal Stream Turbine Analysis. Energies 2015;8:7833-7853.

[52] Malki, R., Williams, A., Croft, T., Togneri, M., Masters, I.. A coupled blade element momentum - Computational fluid dynamics model for evaluating tidal stream turbine performance. Applied Mathematical Modelling 2013;37(5):3006-3020.

[53] Masters, I., Malki, R., Williams, A., Croft, T.. The influence of flow acceleration on tidal stream turbine wake dynamics: A numerical study using a coupled BEM-CFD model. Applied Mathematical Modelling 2013;37(16-17):7905-7918. 
[54] Malki, R., Masters, I., Williams, A., Croft, T.. The variation in wake structure of a tidal stream turbine with flow velocity. In: 4th International Conference on Computational Methods in Marine Engineering, MARINE 2011. Lisbon, Portugal; 2011, p. 1-12.

[55] Edmunds, M., Malki, R., Williams, A., Masters, I., Croft, T.. Aspects of tidal stream turbine modelling in the natural environment using a coupled BEM-CFD model. International Journal of Marine Energy 2014;7:20-42.

[56] Malki, R., Masters, I., Williams, A., Nick Croft, T.. Planning tidal stream turbine array layouts using a coupled blade element momentum - computational fluid dynamics model. Renewable Energy 2014;63:4654 .

[57] Bai, G., Li, J., Fan, P., Li, G.. Numerical investigations of the effects of different arrays on power extractions of horizontal axis tidal current turbines. Renewable Energy 2013;53:180-186.

[58] Schluntz, J., Willden, R.H.J.. An actuator line method with novel blade flow field coupling based on potential flow equivalence. Wind Energy 2015;18(June 2014):1469-1485.

[59] Churchfield, M., Li, Y., Moriarty, P.. A Large-Eddy Simulation Study of Wake Propagation and Power Production in an Array of Tidal-Current Turbines. In: 9th European Wave and Tidal Energy Conference. July; 2011,.

[60] Andersen, P.B., Gaunaa, M., Bak, C.. Validation of an Actuator Line Model Coupled to a Dynamic Stall Model for Pitching Motions Characteristic to Vertical Axis Turbines. In: Journal of Physics: Conference Series 753. 2016, p. 22-43.

[61] McNaughton, J., Afgan, I., Apsley, D., Rolfo, S., Stallard, T., Stansby, P.K.. A simple sliding-mesh interface procedure and its application to the CFD simulation of a tidal-stream turbine. International Journal for Numerical Methods in Fluids 2013; 
[62] McNaughton, J., Rolfo, S., Apsley, D., Afgan, I., Stansby, P., Stallard, T.. CFD prediction of turbulent flow on an experimental tidal stream turbine using RANS modelling. 1st Asian Wave and Tidal Conference Series 2012;.

[63] Baltazar, J., Falcao de Campos, J.. Hydrodynamic Analysis of a Horizontal Axis Marine Current Turbine With a Boundary Element Method. Journal of Offshore Mechanics and Arctic Engineering $2011 ; 133(4): 041304$.

[64] Kang, S., Borazjani, I., Colby, J.a., Sotiropoulos, F.. Numerical simulation of 3D flow past a real-life marine hydrokinetic turbine. Advances in Water Resources 2012;39:33-43.

[65] Jo, C.H., Lee, J.H., Rho, Y.H., Lee, K.H.. Performance analysis of a HAT tidal current turbine and wake flow characteristics. Renewable Energy 2013;65:175-182.

[66] Afgan, 1., McNaughton, J., Rolfo, S., Apsley, D., Stallard, T., Stansby, P.. Turbulent flow and loading on a tidal stream turbine by LES and RANS. International Journal of Heat and Fluid Flow $2013 ; 43: 96-108$.

[67] Schluntz, J., Willden, R.. The effect of blockage on tidal turbine rotor design and performance. Renewable Energy 2015;81:432-441.

[68] Kolekar, N., Banerjee, A.. Performance characterization and placement of a marine hydrokinetic turbine in a tidal channel under boundary proximity and blockage effects. Applied Energy 2015;148:121-133.

[69] Bramwell, F.H., Relfand, E.F., Bryant, W.. Experiments to determine the lateral force on a propeller in a side wind. Tech. Rep.; A.R.C, R. \& M. $123 ; 1914$.

[70] Wood, R.M., Harris, R.G.. Some notes on the theory of an airscrew working in a wind channel. Tech. Rep.; A.R.C., R. \& M. 662; 1920. 
[71] Glauert, H.. Wind Tunnel Interference on Wings, Bodies and Airserews. Tech. Rep. R. \& M. No.1566; 1933.

[72] Ryi, J., Rhee, W., Chang Hwang, U., Choi, J.S.. Blockage effect correction for a scaled wind turbine rotor by using wind tunnel test data. Renewable Energy 2015;79:227-235.

[73] Barnsley, M., Wellicome, J.. Final report on the 2nd phase of development and testing of a horizontal axis wind turbine test rig for the investigation of stall regulation aerodynamics. Carried out under ETSU Agreement E.5A/CON5103/1746. 1990.

|74| Lust, E.E., Luznik, L., Flack, K.A., Walker, J.M., Van Benthem, M.C.. The influence of surface gravity waves on marine current turbine performance. International Journal of Marine Energy 2013;3-4:27-40.

[75] Maskell, E.C.. A Theory of Blockage Effects on Bulff Bodies and Stalled Wings in a Closed Wind Tunnel. Tech. Rep.; 1965.

|76| Whelan, J.I., Graham, J.M.R., Peiró, J.. A free-surface and blockage correction for tidal turbines. Journal of Fluid Mechanics 2009;624:281.

[77] Birjandi, A.H., Bibeau, E.L., Chatoorgoon, V., Kumar, A.. Power measurement of hydrokinetic turbines with free-surface and blockage effect. Ocean Engineering 2013;69:9-17.

[78] Chen, T.Y., Liou, L.R.. Blockage corrections in wind tunnel tests of small horizontal-axis wind turbines. Experimental Thermal and Fluid Science 2011;35(3):565-569.

[79] Medici, D., Ivanell, S., Dahlberg, J.A., Alfredsson, P.H.. The upstream flow of a wind turbine: Blockage effect. Wind Energy 2011;14(January):691-697.

[80] Myers, L., Bahaj, A.. An experimental investigation simulating flow effects in first generation marine current energy converter arrays. Renewable Energy 2012;37(1):28-36. 
[81] McTavish, S., Rodrigue, S., Feszty, D., Nitzsche, F.. An investigation of in-field blockage effects in closely spaced lateral wind farm configurations. Wind Energy 2015;18(September 2014):1989-2011.

[82] Meyer Forsting, a.R., Troldborg, N.. The effect of blockage on power production for laterally aligned wind turbines. Journal of Physics: Conference Series 2015;625:012029.

[83] Nishino, T., Willden, R.. The efficiency of an array of tidal turbines partially blocking a wide channel. Journal of Fluid Mechanics 2012;708:596-606.

[84] Nishino, T., Willen, R.. Two-scale dynamics of flow past a partial cross-stream array of tidal turbines. Journal of Fluid Mechanics 2013;730:220-244.

[85] Nishino, T., Draper, S.. Local blockage effect for wind turbines. Journal of Physics: Conference Series 2015;012010:10.

[86] Houlsby, G.T., Draper, S., Oldfield, M.L.G.. Application of Linear Momentum Actuator Disc Theory to Open Channel Flow by. Tech. Rep.; OUEL 2296/08, Department of Engineering Science, University of Oxford; 2008.

[87] Werle, M.J.. Wind Turbine Wall-Blockage Performance Corrections. Journal of Propulsion and Power 2010;26(6):1317-1321.

[88] Chamorro, L.P., Troolin, D.R., Lee, S.J., Arndt, R.E.a., Sotiropoulos, F.. Three-dimensional flow visualization in the wake of a miniature axial-flow hydrokinetic turbine. Experiments in Fluids 2013;j4.

[89] Neary, V.S., Gunawan, B., Hill, C., Chamorro, L.P.. Near and far field flow disturbances induced by model hydrokinetic turbine: ADV and ADP comparison. Renewable Energy 2013;60:1 6.

[90] Tedds, S.C., Owen, I., Poole, R.J.. Near-wake characteristics of a model horizontal axis tidal stream turbine. Renewable Energy 2014;63:222-235. 
[91] Bahaj, A.S., Myers, L.E.. Shaping array design of marine current energy converters through scaled experimental analysis. Energy 2013;59:83-94.

[92] McCombes, T., Johnstone, C., Grant, a.. Unsteady 3D Wake Modelling for Marine Current Turbines. Proceedings of the 8th European Wave and Tidal Energy Conference, Uppsala, Sweden 2009;:548-557.

[93] Myers, L., Bahaj, A.. Wake studies of a 1/30th scale horizontal axis marine current turbine. Ocean Engineering 2007;34(5-6):758-762.

[94] Maganga, F., Germain, G., King, J., Pinon, G., Rivoalen, E.. Experimental Characterisation of flow effects on marine current turbine behaviour and on its wake properties. IET Renewable Power Generation 2010;4(6):498-509.

[95] Myers, L., Bahaj, A.. Near wake properties of horizontal axis marine current turbines. In: 8th European Wave and Tidal Energy Conference. Uppsala; 2009, p. 558--565.

(96) Stallard, T., Collings, R., Feng, T., Whelan, J.. Interactions betweem tidal turbine wakes: Experimental study of a group of 3bladed rotors. Philosophical Transactions of the Royal Society of London: A 2013;371:1471-2962.

[97] Myers, L.E., Bahaj, A.S.. Experimental analysis of the flow field around horizontal axis tidal turbines by use of scale mesh disk rotor simulators. Ocean Engineering 2010;37(2-3):218-227.

[98] Bahaj, A.S., Myers, L.E., Rawlinson-Smith, R.I., Thomson, M.. The Effect of Boundary Proximity Upon the Wake Structure of Horizontal Axis Marine Current Turbines. Journal of Offshore Mechanics and Arctic Engineering 2012;134(2):021104.

[99] Sanderse, B.. Aerodynamics of wind turbine wakes: Literature review. Tech. Rep.; ECN-E-09-016; 2009. 
[100] Mo, J.O., Choudhry, A., Arjomandi, M., Lee, Y.H.. Large eddy simulation of the wind turbine wake characteristics in the numerical wind tunnel model. Journal of Wind Engineering and Industrial Acrodynamics 2013;112:11-24.

[101] Troldborg, N., Sorensen, J.N., Mikkelsen, R.. Numerical simulations of wake characteristics of a wind turbine in uniform inflow. Wind Energy 2010;13(June 2009):86-99.

[102] Moriarty, P.J.. AeroDyn Theory Manual. 2005.

[103] Lanzafame, R., Messina, M.. Fluid dynamics wind turbine design: Critical analysis, optimization and application of BEM theory. Renewable Energy 2007;32(14):2291-2305.

[104] Divett, T., Vennell, R., Stevens, C.. Channel scalc tuning of large tidal turbine arrays using large eddy simulations with adaptive mesh. EWTEC 2013 Proceedings 2013;86:1394-1405. URL: http://dx. doi.org/10.1016/j.renene.2015.09.048. doi:10.1016/j.renene. 2015.09 .048 .

[105] Rao, S., Xue, H., Bao, M., Funke, S.. Determining tidal turbine farm efficiency in the Western Passage using the disc actuator theory. Ocean Dynamics 2016;66:41-57.

[106] ANSYS, . ANSYS FLUENT 14 Theory Guide. 2011.

[107] Celik, I., Ghia, U., Roache, P., Freitas, C.. Procedure for Estimation and Reporting of Uncertainty Due to Discretization in CFD Applications. Journal of Fluids Engineering 2008;130(7).

[108] ANSYS, . ANSYS FLUENT 14.0 User's Guide. 2011.

[109] Mozafari, A.T.J.. Numerical Modeling of Tidal Turbines : Methodology Development and Potential Physical Environmental Effects. Master's thesis; University of Washington; 2010. 
[110] Mason-Jones, A.. Performance assessment of a Horizontal Axis Tidal Turbine in a high velocity shear environment. Phd thesis; Cardiff University, UK; 2010.

[111] Drela, M.. XFOIL: An analysis and design system for low Reynolds number airfoils. In: Low Reynolds number aerodynamics. University of Notre Dame, USA; 1989, p. 1-12.

[112] Drela, M., Youngren, H.. XFOIL 6.9 User Primer. 2001. URL: http://web.mit.edu/aeroutil】_v1.0/xfoil】_doc.txt.

[113] Ananda, G., Sukumar, P., Selig, M.. Low-to-Moderate aspect ratio wings tested at low Reynolds number. In: 30th AIA A Applied Aerodynamics Conference. June; New Orleans, Louisiana; 2012, p. 1-19.

[114] Selig, M.S., Mcgranahan, B.D.. Wind tunnel aerodynamic tests of six airfoils for use on small wind turbines. Tech. Rep. October; NREL/SR-500-34515; 2004.

[115] Jahanmiri, M.. Laminar Separation Bubble: Its Structure, Dynamics and Control. Tech. Rep.; Chalmers University of Technology; 2011.

|116| Viterna, L., Corrigan, R.. Fixed pitch rotor performance of large horizontal axis wind turbines. Tech. Rep.; NASA Lewis Research Centre; Cleveland, Ohio; 1981.

[117] Keogh, B., Myers, L., Bahaj, A.S.. Tidal stream turbine device performance with changes in channel size and geometry. In: 2nd Asian Wave and Tidal Energy Conference. Tokyo; 2014,.

[118] Giles, J., Myers, L., Bahaj, A., Shelmerdine, B.. The downstream wake response of marine current energy converters operating in shallow tidal flows. In: World Renewable Energy Congress. Linkoping; 2011,.

[119] Blackmore, T., Myers, L.E., Bahaj, A.S.. International Journal of Marine Energy Effects of turbulence on tidal turbines: Implications to performance, blade loads, and condition monitoring. International Journal of Marine Energy 2016;14:1-26. 
[120] Peña, A., Réthoré, P.E., Rathmann, O.. Modeling large offshore wind farms under different atmospheric stability regimes with the Park wake model. Renewable Energy 2014;70:164-171.

[121] Barthelmie, R.J., Folkerts, L., Larsen, G.C., Rados, K., Pryor, S.C., Frandsen, S.T., et al. Comparison of wake model simulations with offshore wind turbine wake profiles measured by sodar. Journal of Atmospheric and Oceanic Technology 2006;23:888-901.

[122] Palm, M., Huijsmans, R., Pourquie, M.. The Applicability of SemiEmpirical Wake Models for Tidal Farms. In: 9th European Wave and Tidal Energy Conference. Southampton; 2011,.

[123| Frandsen, S., Barthelmie, R., Pryor, S., Rathmann, O., Larsen, S., Hojstrup, J.. Analytical modelling of wind speed deficit in large offshore wind farms. Wind Energy 2006;9(January):39-53.

[124] Vennell, R.. Tuning turbines in a tidal channel. Journal of Fluid Mechanics 2010;663:253-267.

[125] Vennell, R.. The energetics of large tidal turbine arrays. Renewable Energy 2012;48:210-219.

[126] Vennell, R.. Realizing the potential of tidal currents and the efficiency of turbine farms in a channel. Renewable Energy 2012;47:95-102. 


\section{Appendix A}

\section{Validation cases contour plots}

\section{A.1 Contour plots for the empty flume}

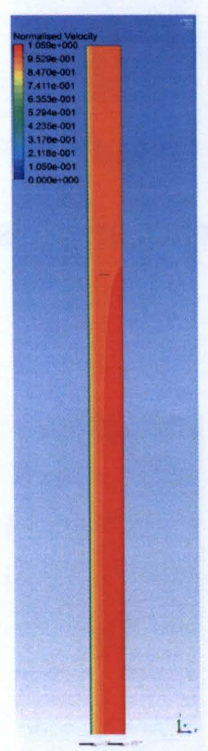

(a) SKE

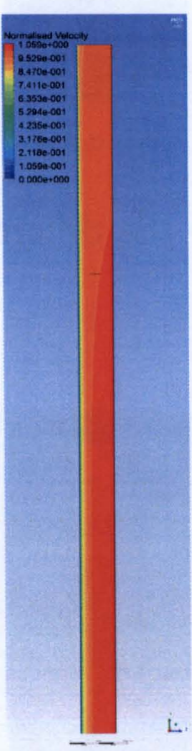

(b) RKE

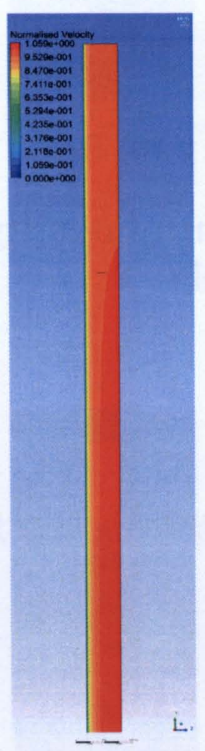

(c) SKW

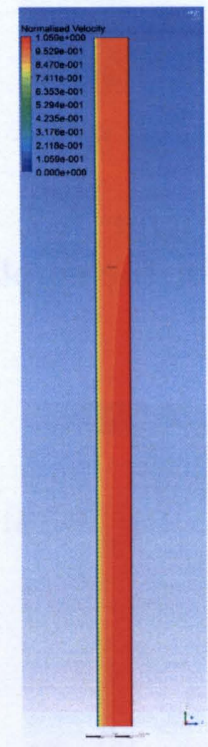

(d) KW-SST

Figure A.1: Velocity plots by the different turbulence models for the empty channel 


\section{Validation cases contour plots}
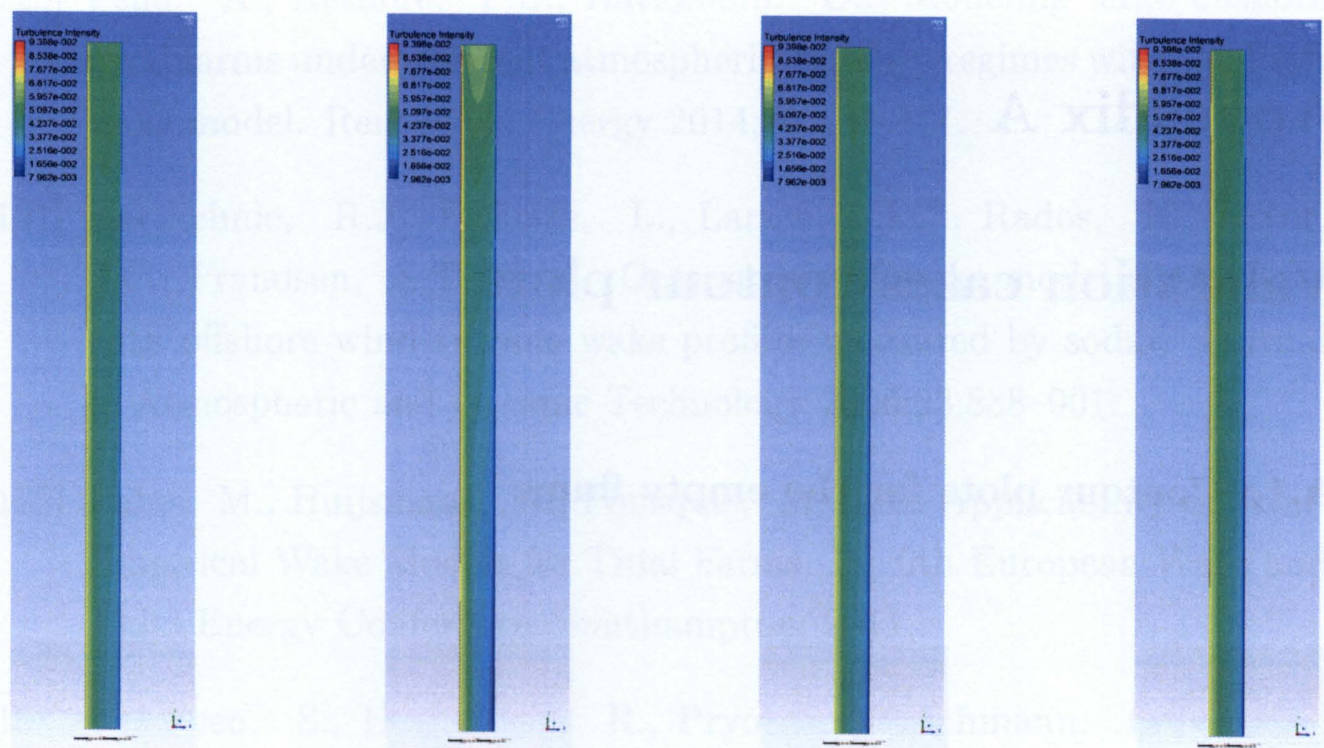

(a) SKE

(b) RKE

(c) SKW

(d)

Figure A.2: Turbulence Intensity plots by the different turbulence models for the empty channel

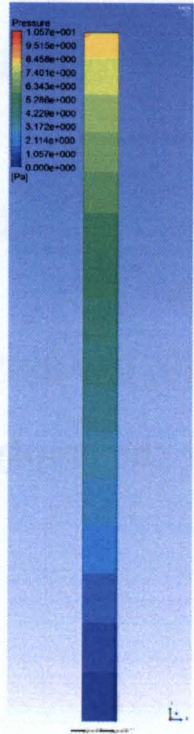

(a) SKE

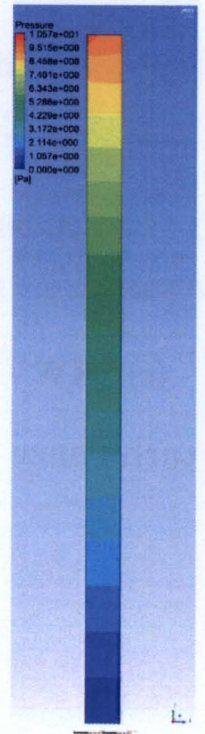

(b) RKE

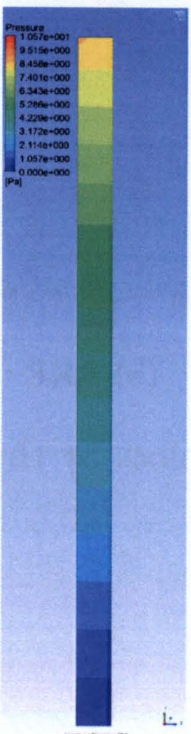

(c) SKW

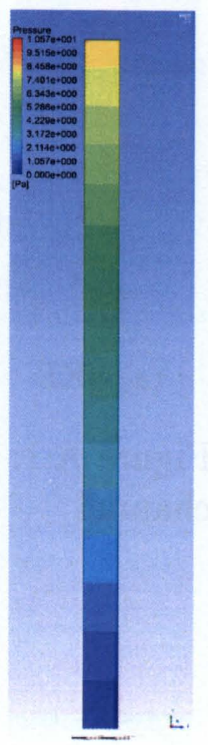

(d)

Figure A.3: Pressure plots by the different turbulence models for the empty channel 


\section{A.2 Contour plots for a turbine in the flume}

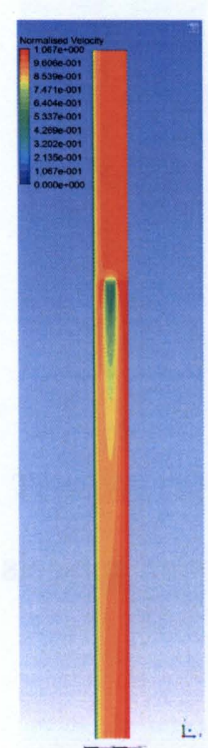

(a) SKE

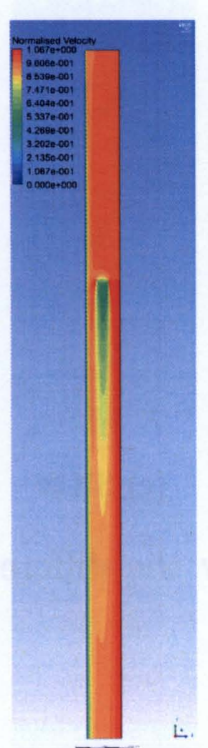

(b) RKE

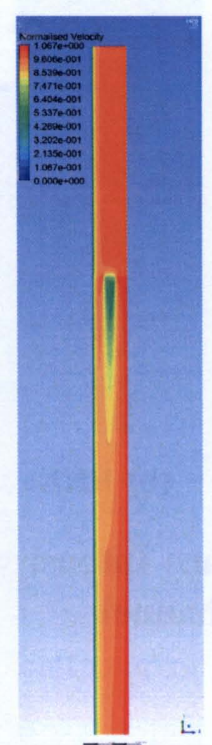

(c) SKW

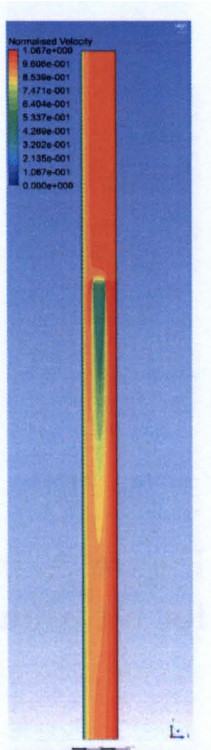

(d) KW-SST

Figure A.4: Velocity plots by the different turbulence models for a turbine in the channel 


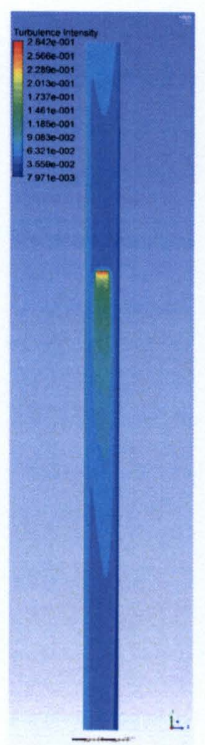

(a) SKE

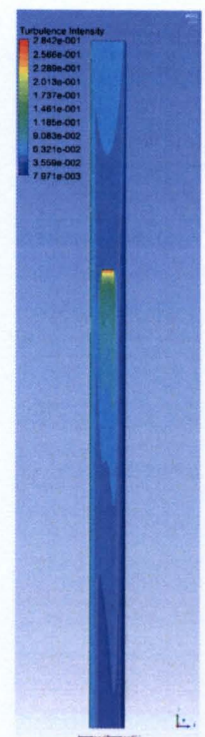

(b) RKE

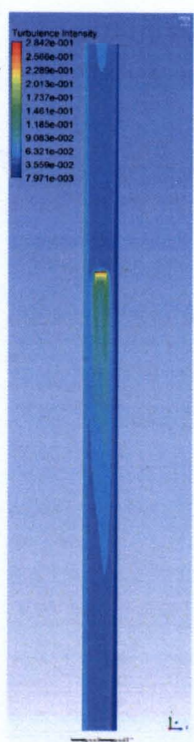

(c) SKW

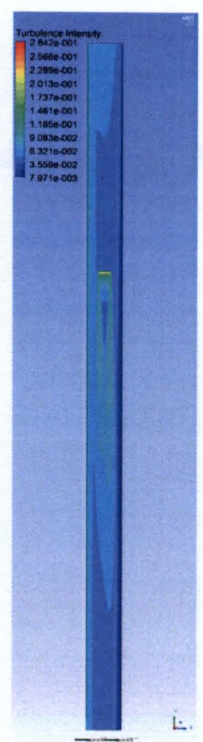

(d) KW-SST

Figure A.5: Turbulence Intensity plots by the different turbulence models for a turbine in the channel

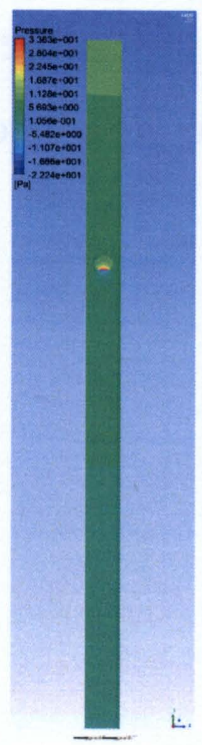

(a) SKE

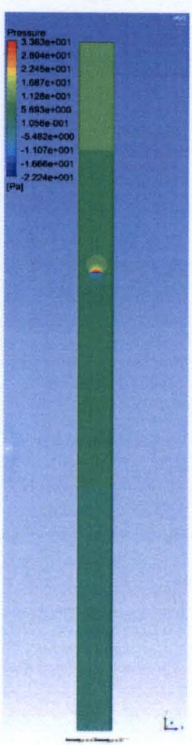

(b) RKE

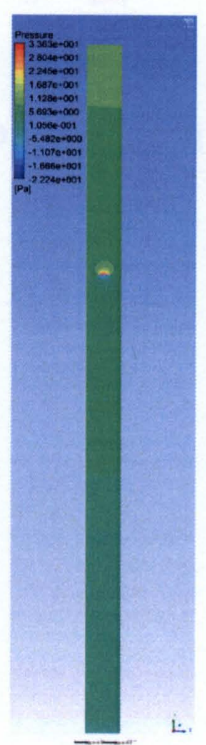

(c) SKW

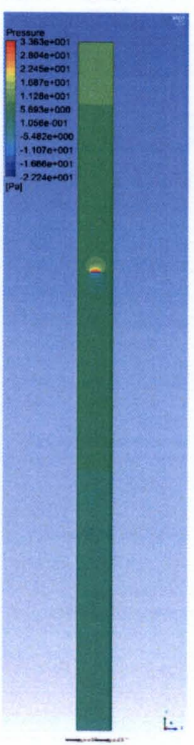

(d) KW-SST

Figure A.6: Pressure plots by the different turbulence models for a turbine in the channel 


\section{A.3 Contour plots for turbines with different added turbulence intensity}

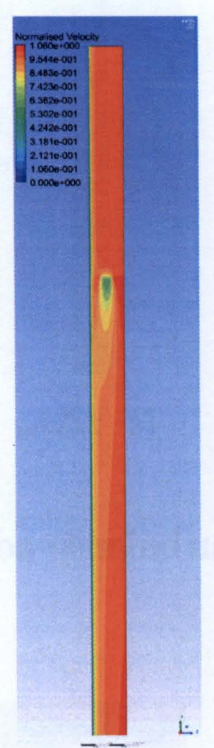

(a) No added turbulence

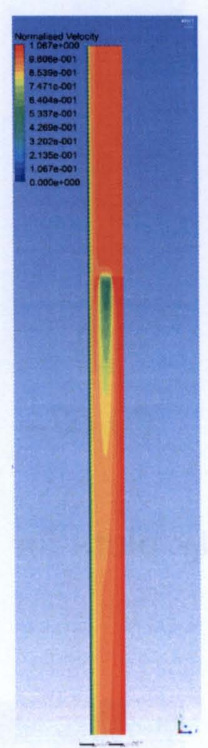

(b) $\triangle I_{m}$

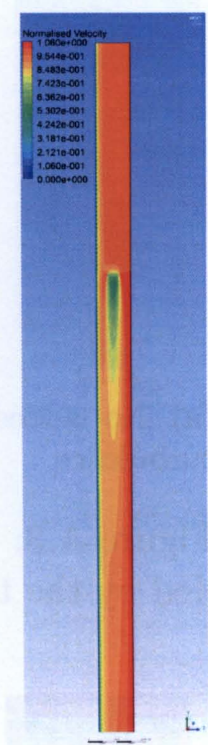

(c) $2 \triangle I_{m}$

Figure A.7: Velocity plots for different levels of turbulence added by the turbine 


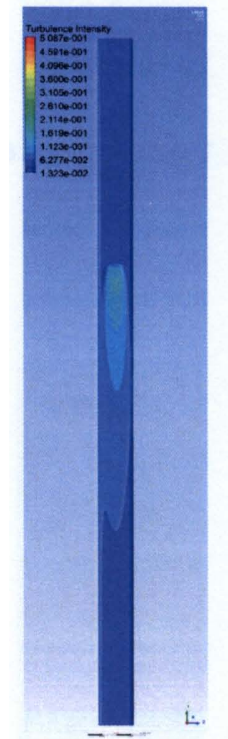

(a) No added turbulence

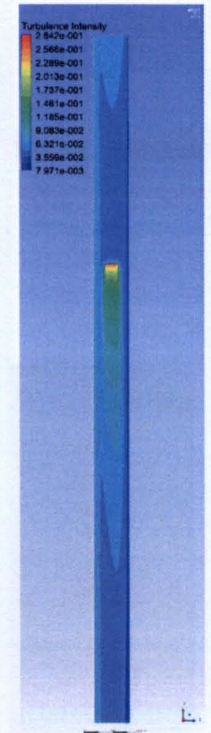

(b) $\triangle I_{m}$

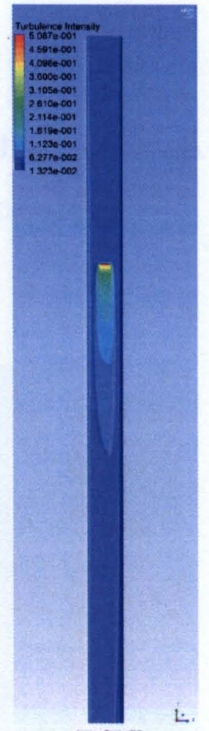

(c) $2 \triangle I_{m}$

Figure A.8: Turbulence Intensity plots for different levels of turbulence added by the turbine

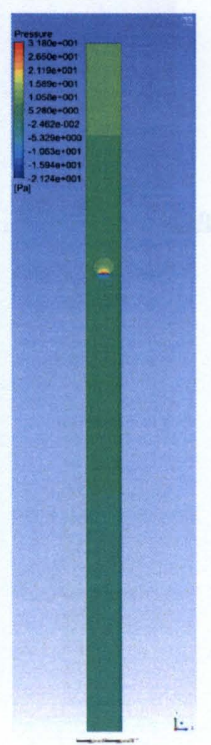

(a) No added turbulence
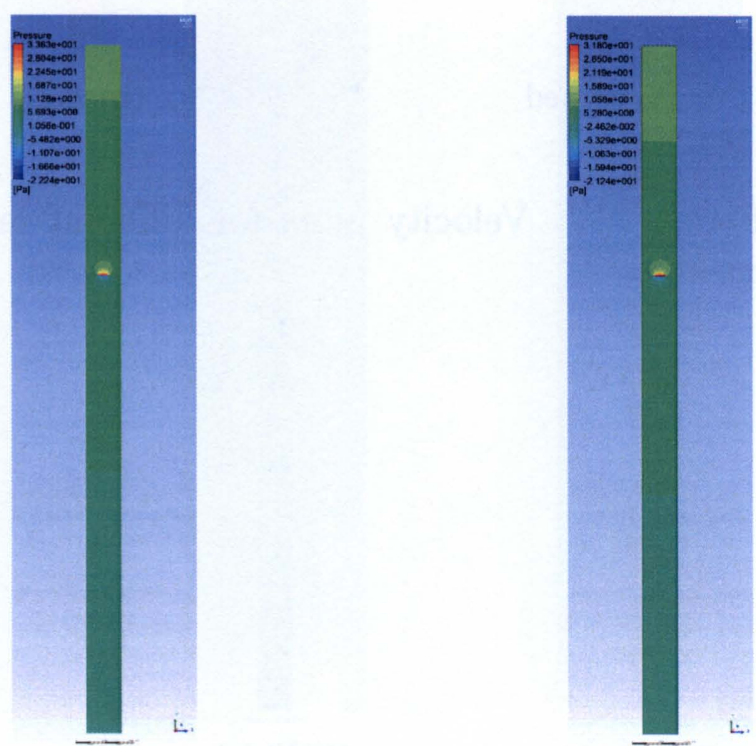

(b) $\triangle I_{m}$

(c) $2 \triangle I_{m}$

Figure A.9: Pressure plots for different levels of turbulence added by the turbine 


\section{Appendix B}

Contour plots for single turbine cases 


\section{B.1 Plots for a single turbine in channels with the boundary layer present}

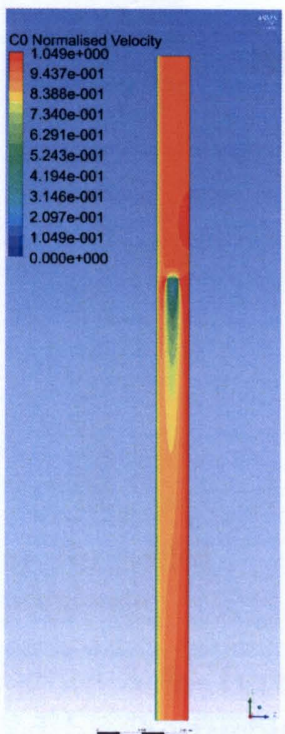

(a) Channel 0

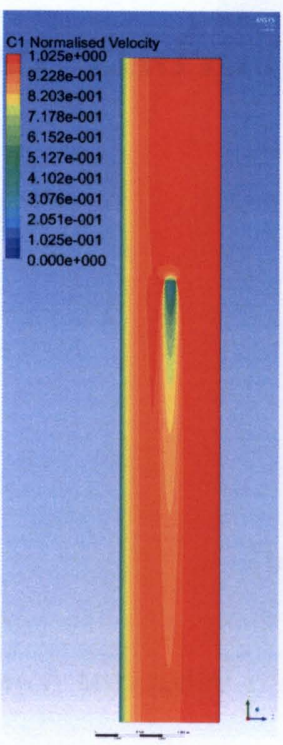

(b) Channel 1

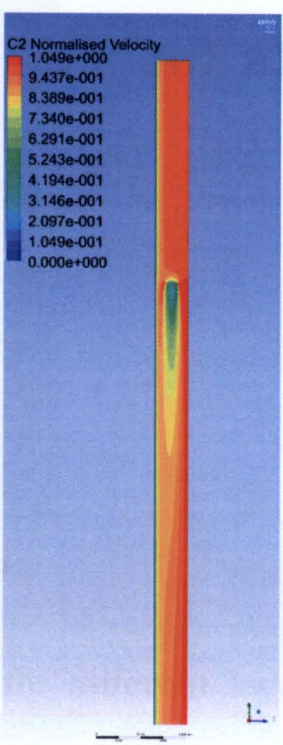

(c) Channel 2

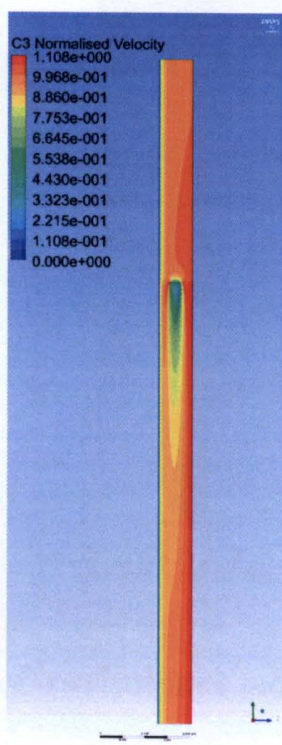

(d) Channel 3

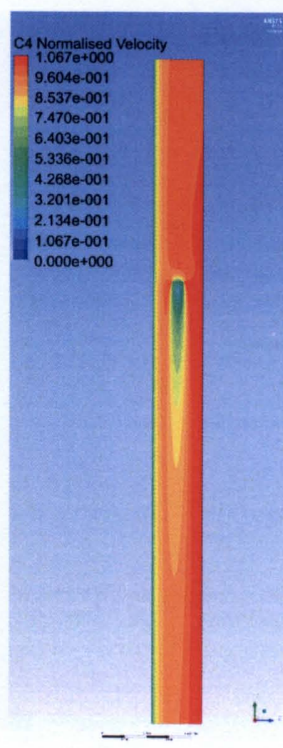

(e) Channel 4

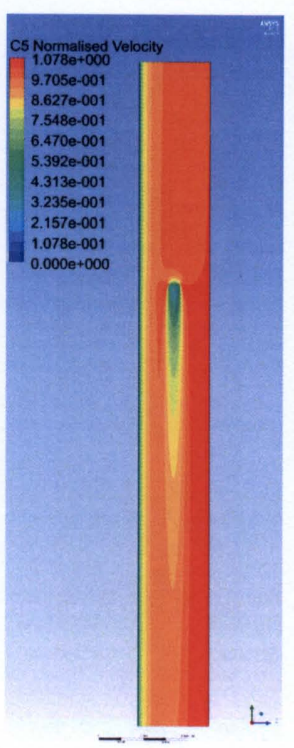

(f) Channel 5

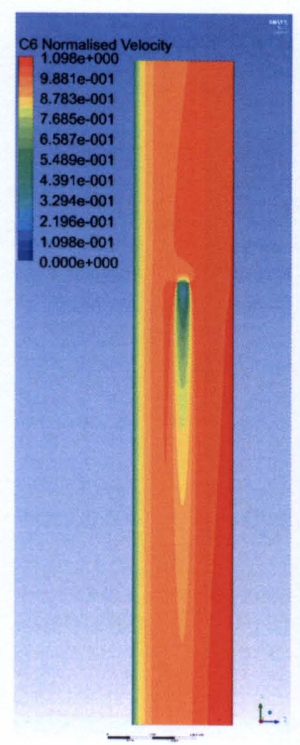

(g) Channel 6

Figure B.1: Velocity plots for the different channels with the boundary layer present 


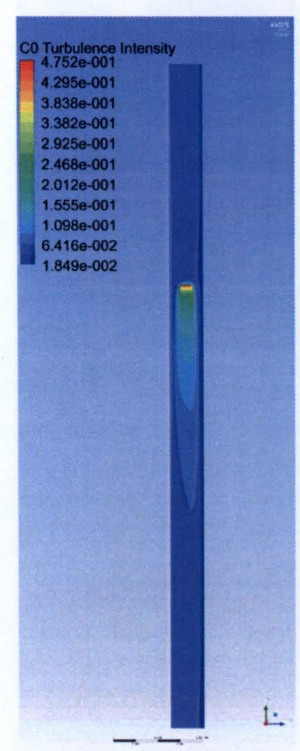

(a) Channel 0

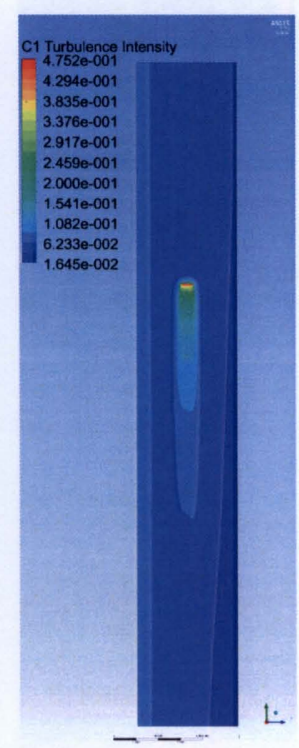

(b) Channel 1

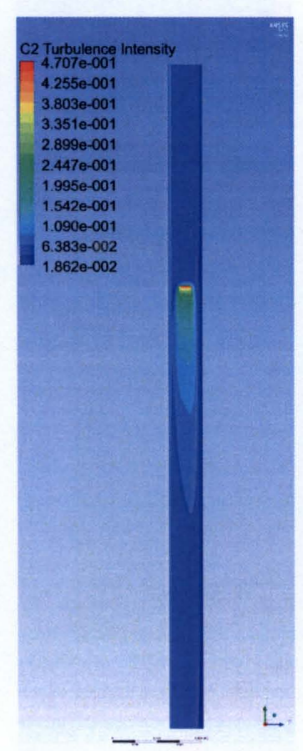

(c) Channel 2

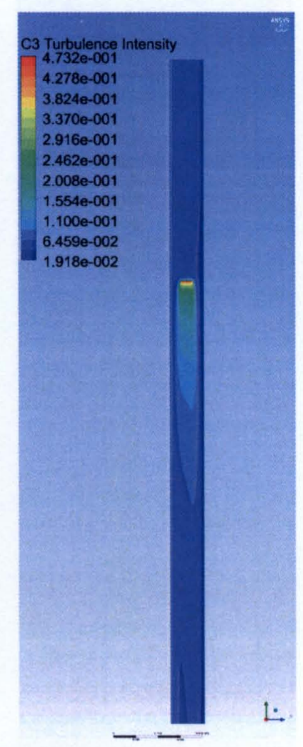

(d) Channel 3

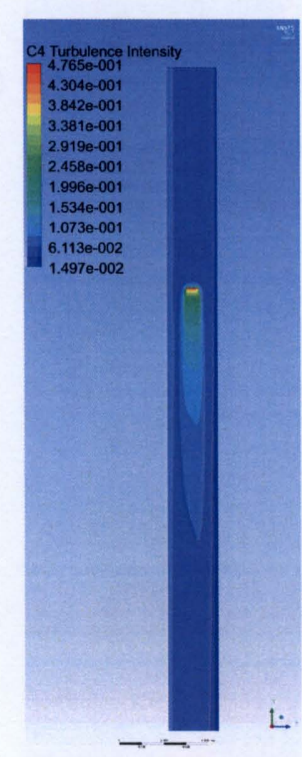

(e) Channel 4

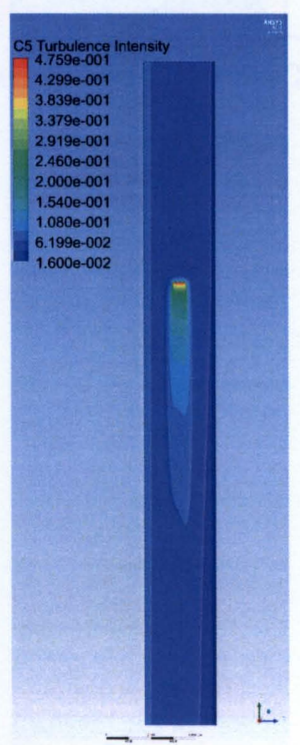

(f) Channel 5

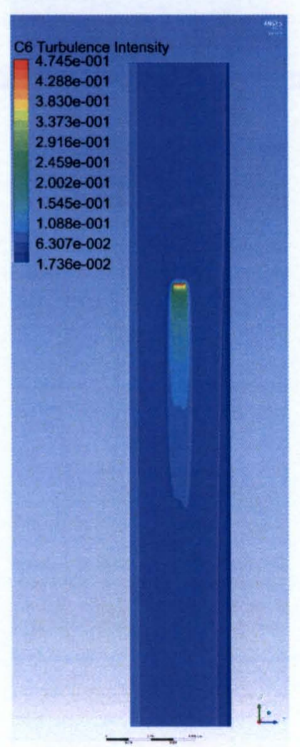

(g) Channel 6

Figure B.2: Turbulence Intensity plots for the different channels with the boundary layer present 


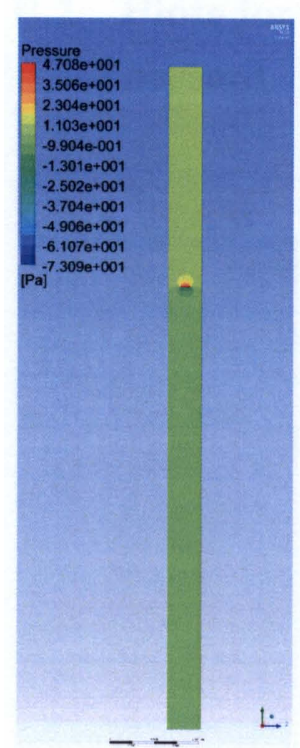

(a) Channel 0

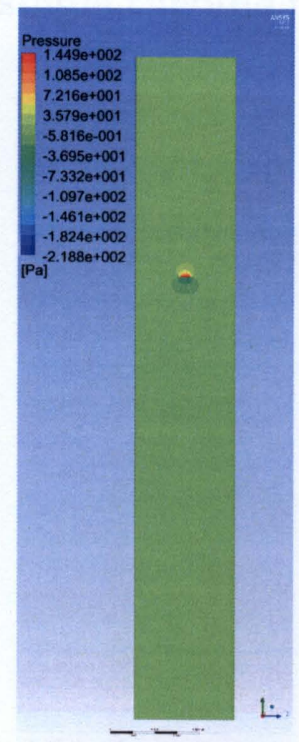

(b) Channel 1

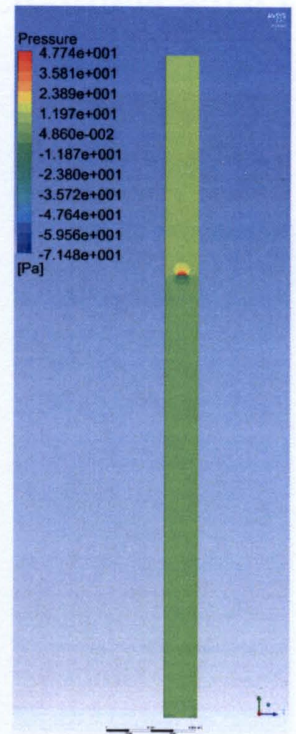

(c) Channel 2

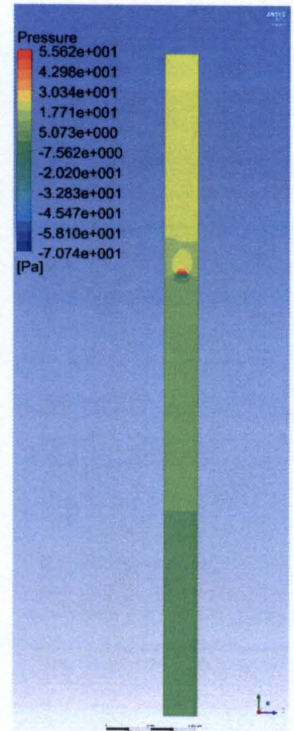

(d) Channel 3

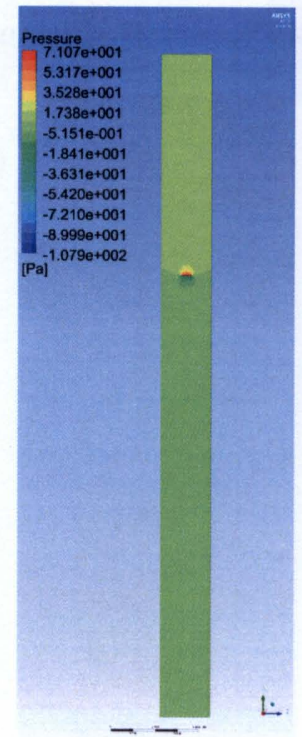

(e) Channel 4

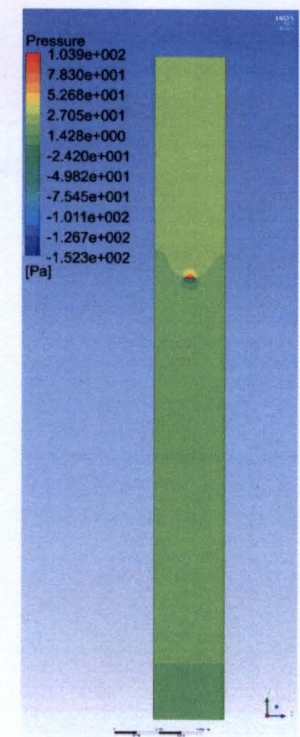

(f) Channel 5

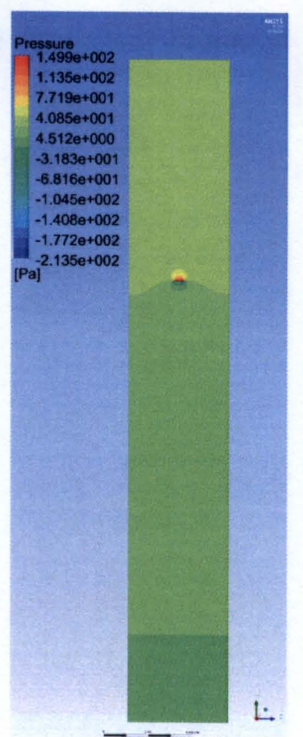

(g) Channel 6

Figure B.3: Pressure plots for the different channels with the boundary layer present 


\section{B.2 Plots for a single turbine in channels without the boundary} layer

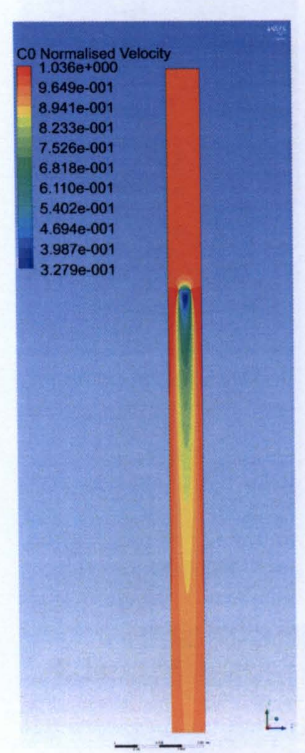

(a) Channel 0

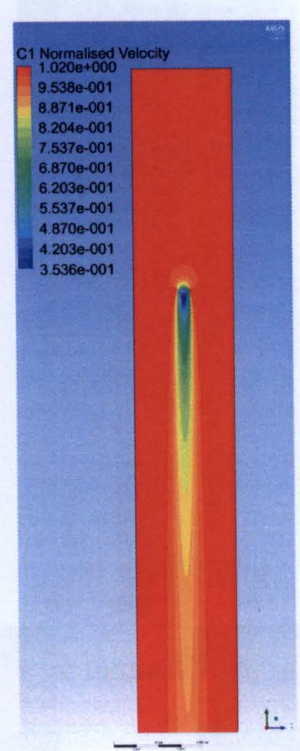

(b) Channel 1

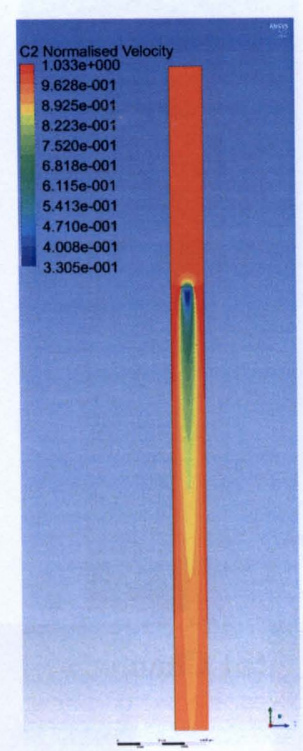

(c) Channel 2

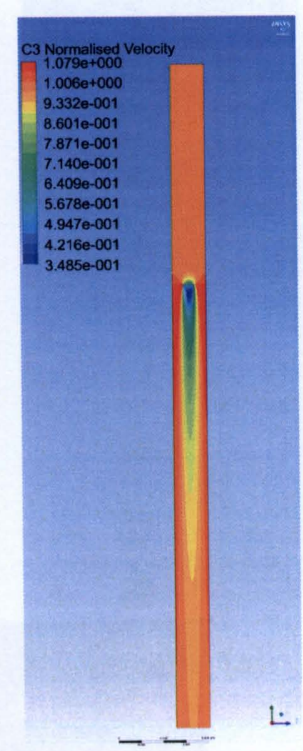

(d) Channel 3

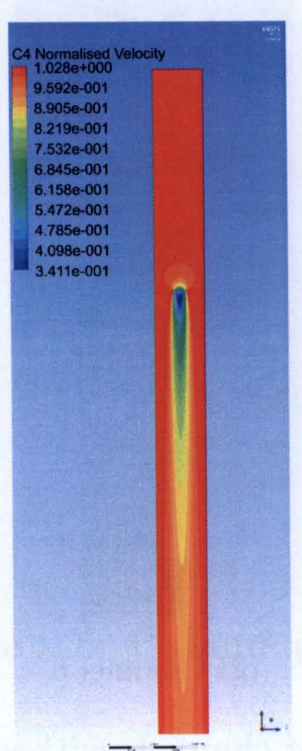

(e) Channel 4

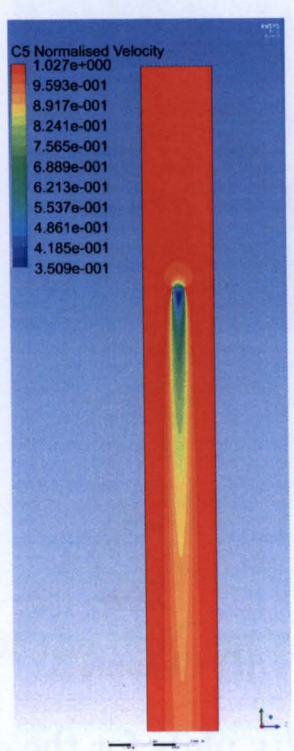

(f) Channel 5

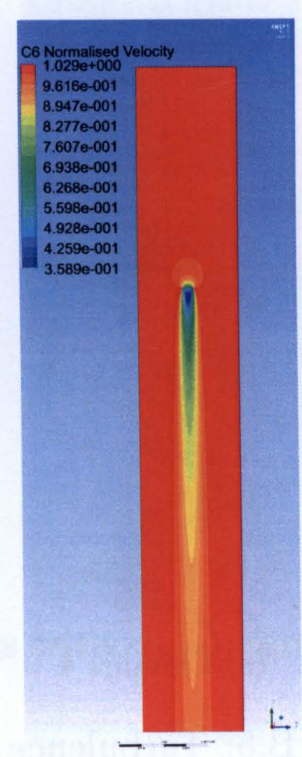

(g) Channel 6

Figure B.4: Velocity plots for the different channels without the boundary layer 

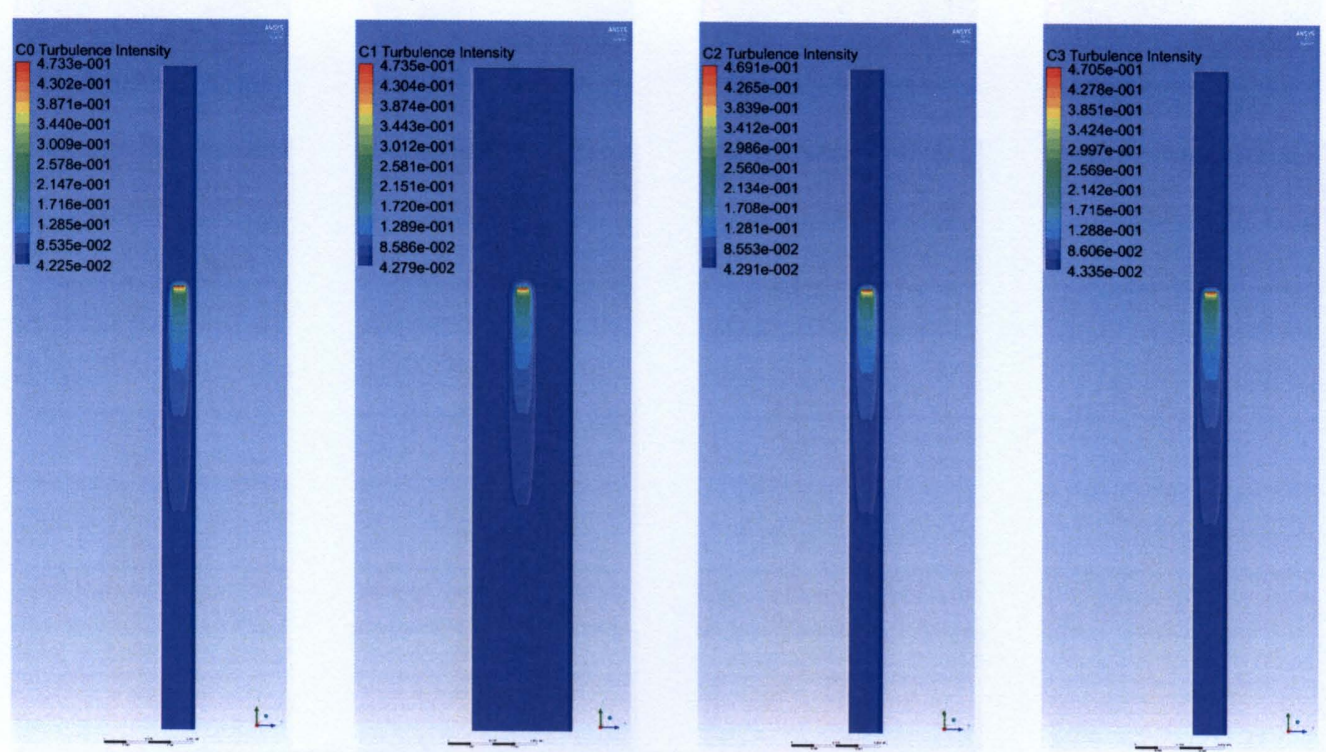

(a) Channel 0

(b) Channel 1

(c) Channel 2

(d) Channel 3

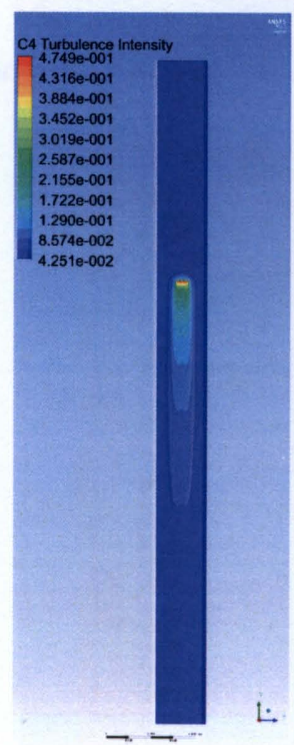

(e) Channel 4

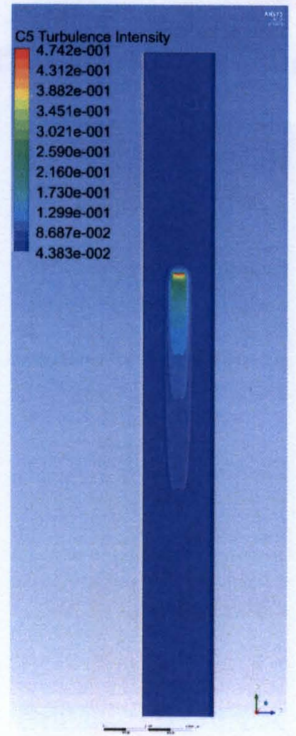

(f) Channel 5

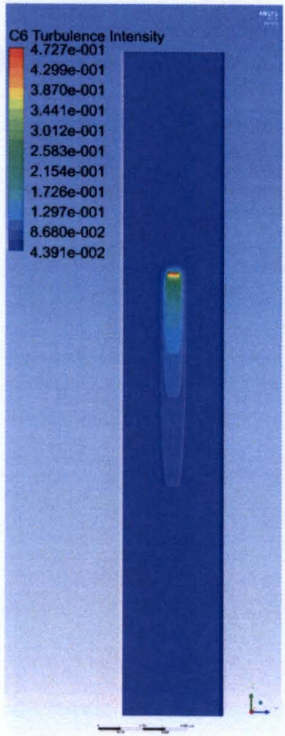

(g) Channel 6

Figure B.5: Turbulence Intensity plots for the different channels without the boundary layer 


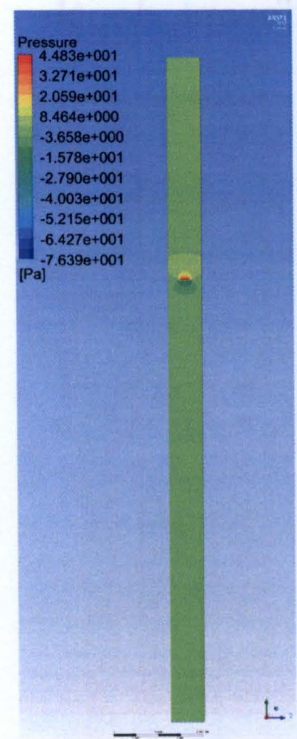

(a) Channel 0

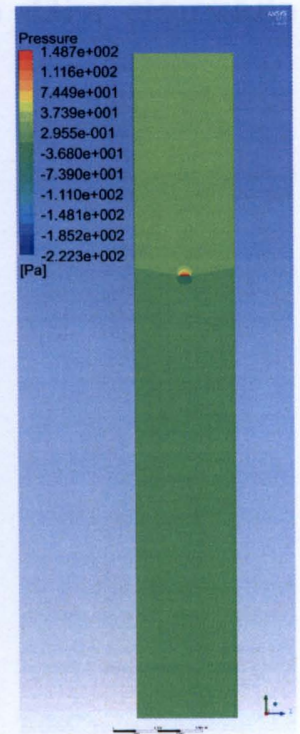

(b) Channel 1

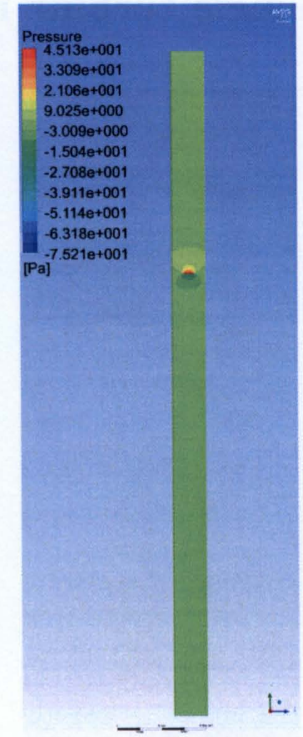

(c) Channel 2

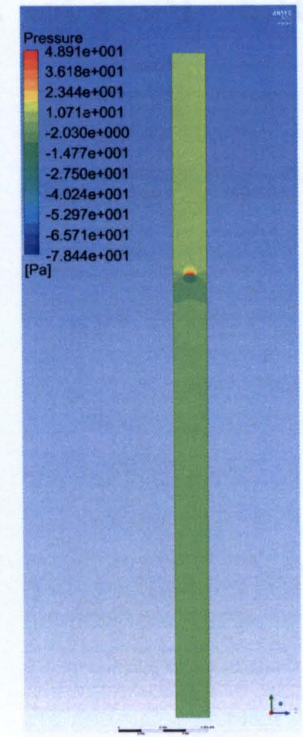

(d) Channel 3

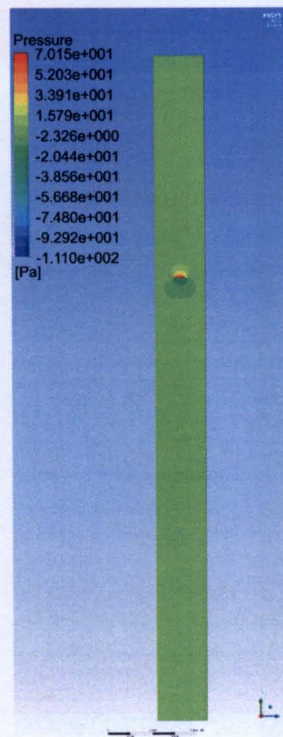

(e) Channel 4

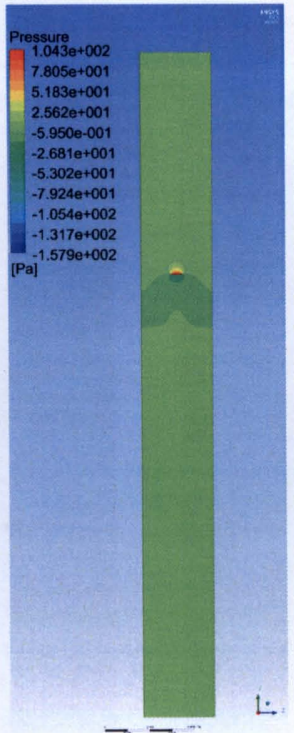

(f) Channel 5

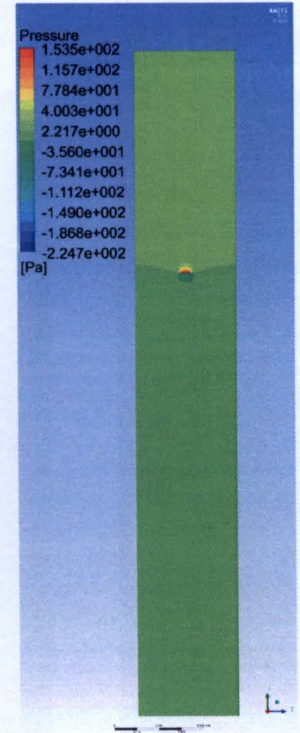

(g) Channel 6

Figure B.6: Pressure plots for the different channels without the boundary layer 


\section{B.3 Plots for a single turbine in channels with different depth}

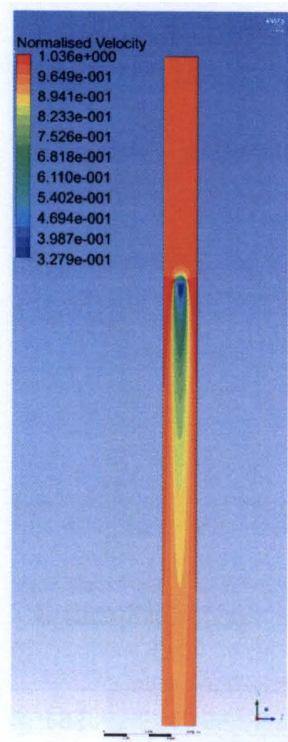

(a)

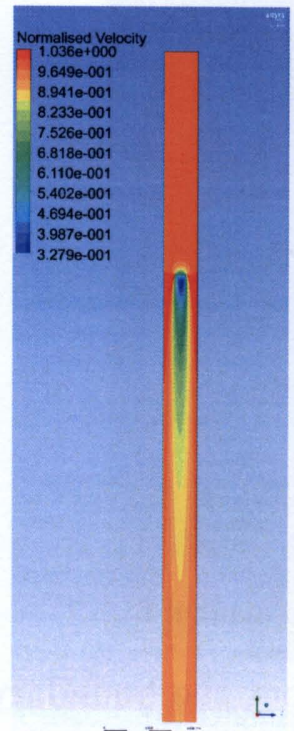

(e) Channel 0

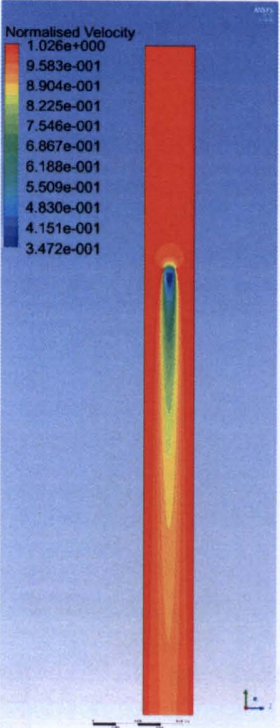

(b)

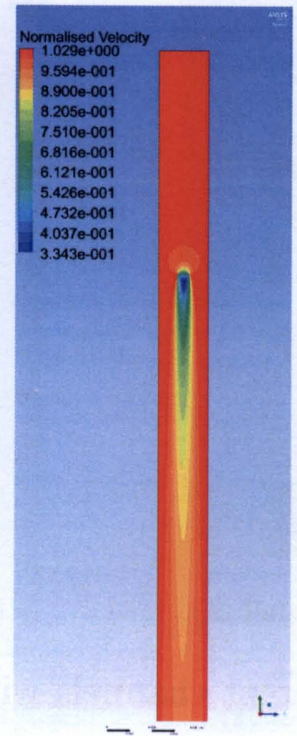

(f) Channel 4

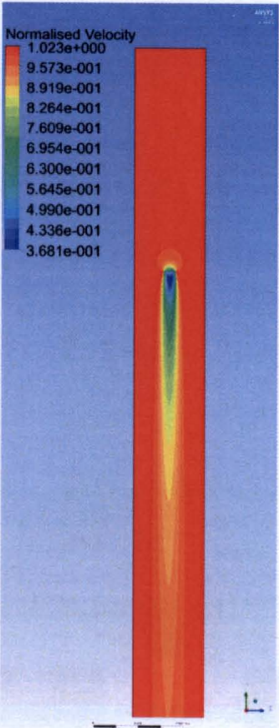

(c)

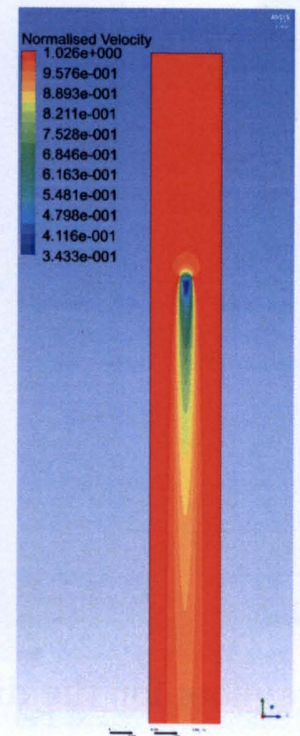

(g) Channel 5

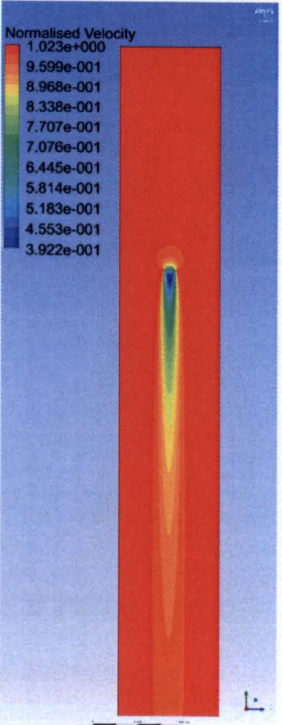

(d)

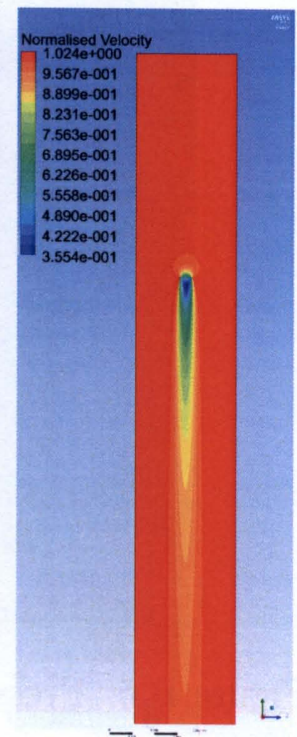

(h) Channel 6

Figure B.7: Velocity contours for channels with varying depth for turbulent length scale varying with depth (a-d), constant turbulent length scale (e-h) 


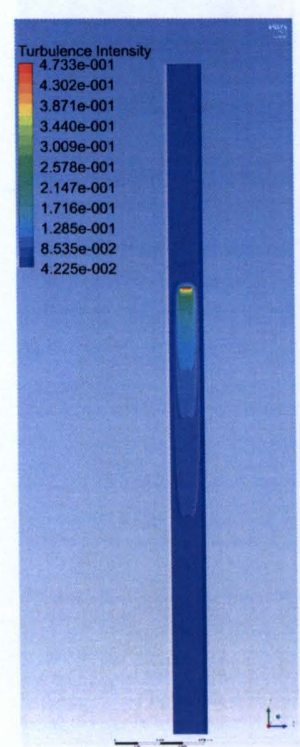

(a)

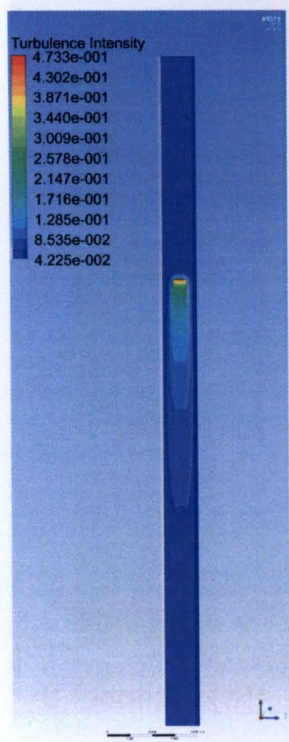

(e) Channel 0

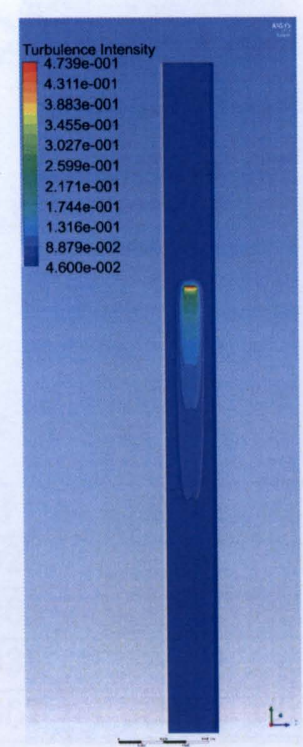

(b)

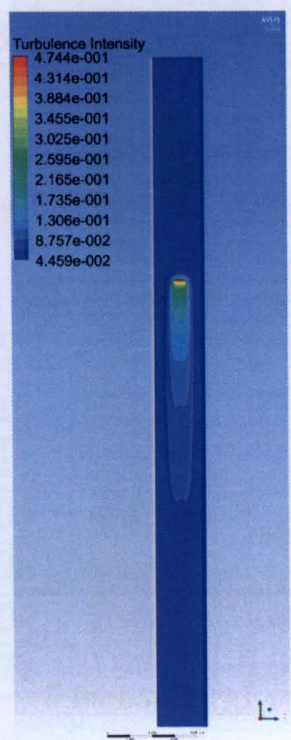

(f) Channel 4

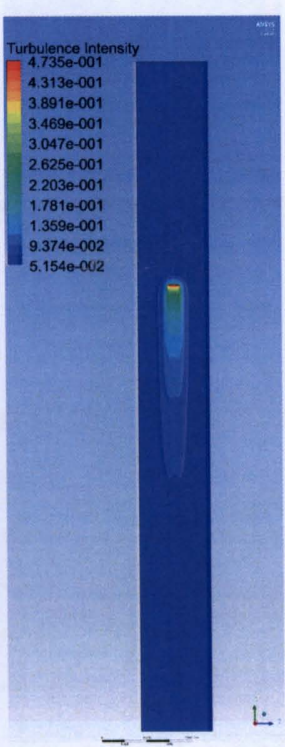

(c)

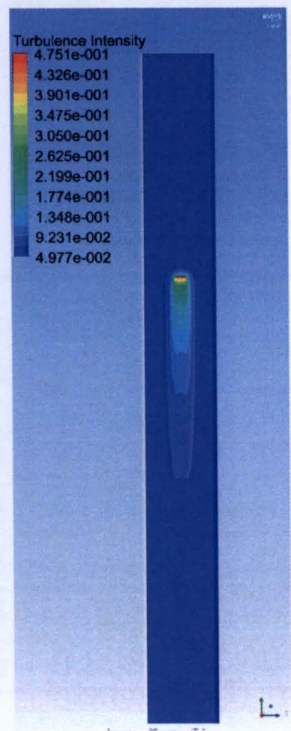

(g) Channel 5

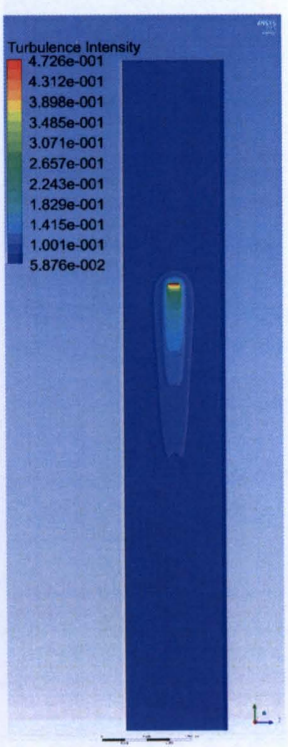

(d)

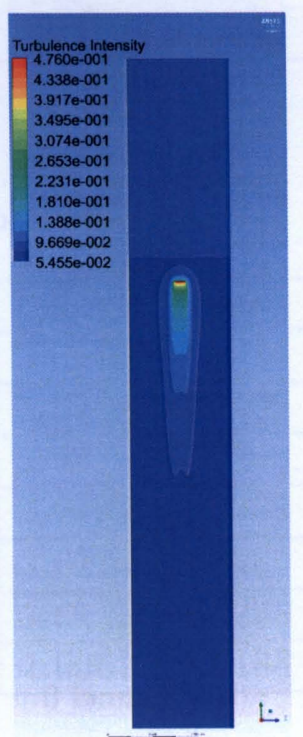

(h) Channel 6

Figure B.8: Turbulence Intensity contours for channels with varying depth for turbulent length scale varying with depth (a-d), constant turbulent length scale $(e-h)$ 
Contour plots for single turbine cases

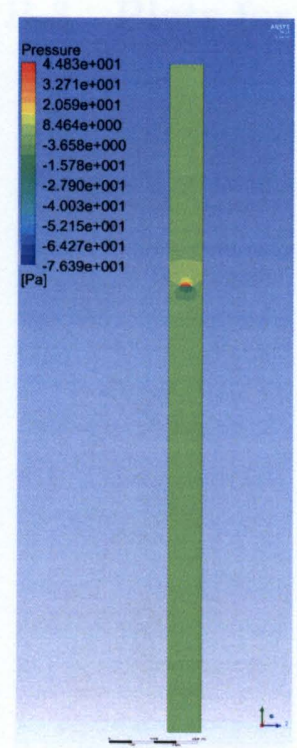

(a)

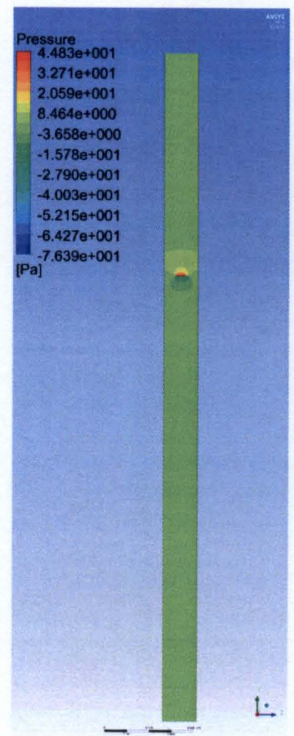

(e) Channel 0

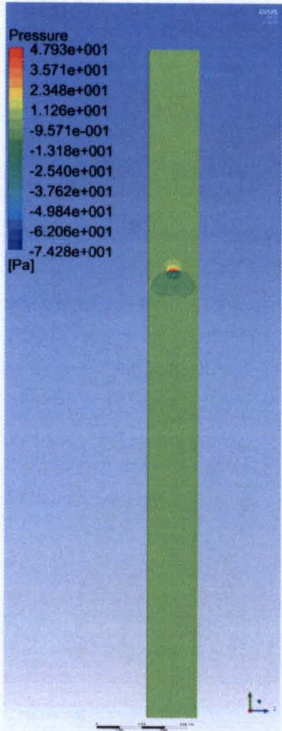

(b)

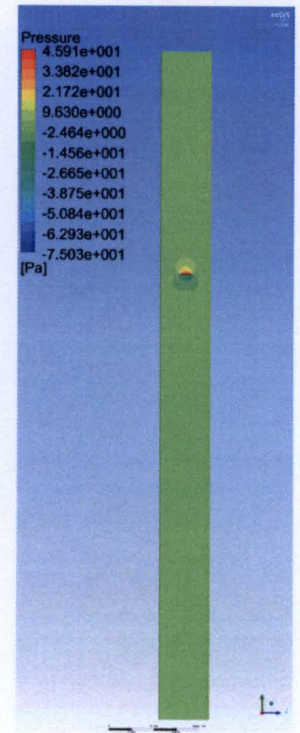

(f) Channel 4

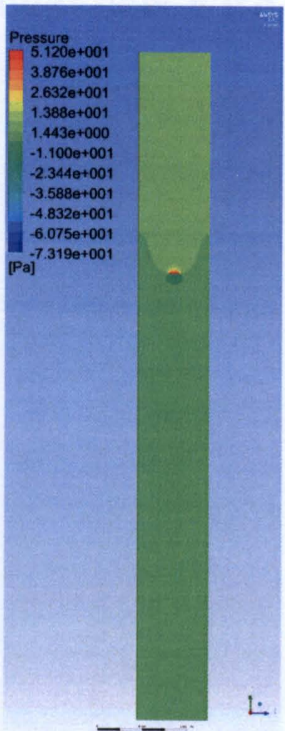

(c)

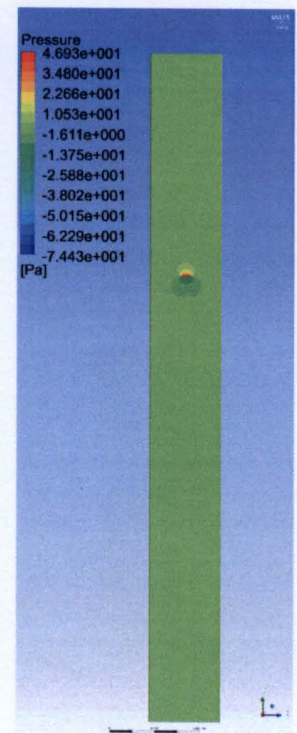

(g) Channel 5

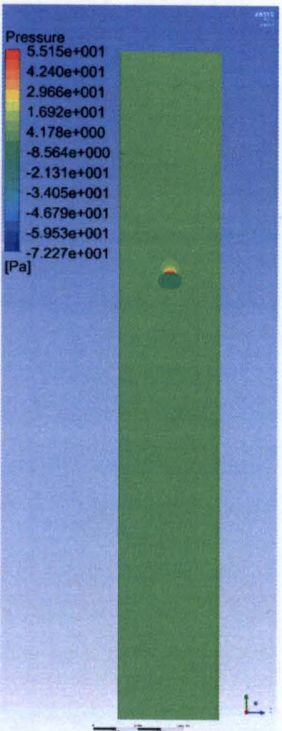

(d)

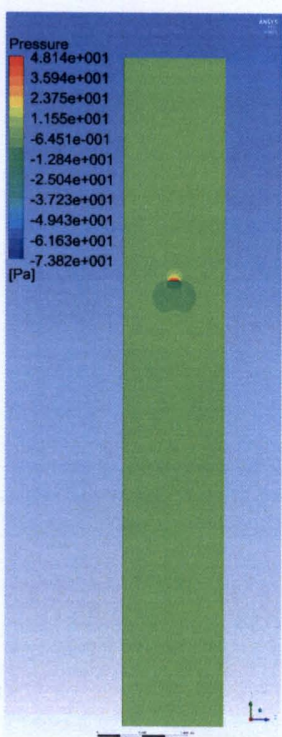

(h) Channel 6

Figure B.9: Pressure contours for channels with varying depth for turbulent length scale varying with depth (a-d), constant turbulent length scale (e-h) 


\section{Appendix C}

\section{Velocity ratios for single turbine case}

Table C.1: Table of velocity ratios for channels without boundary layer

\begin{tabular}{|c|c|c|c|c|c|c|c|r}
\hline Ct & 0.3 & 0.4 & 0.5 & 0.6 & 0.7 & 0.8 & 0.9 & 1 \\
\hline Channel 0 & 1.0028 & 1.0039 & 1.0059 & 1.0093 & 1.0148 & 1.0237 & 1.0398 & 1.1 \\
\hline Channel 1 & 1.0068 & 1.0105 & 1.0153 & 1.0214 & 1.0292 & 1.0402 & 1.0584 & 1.1 \\
\hline Channel 2 & 1.0035 & 1.0051 & 1.0077 & 1.0118 & 1.0180 & 1.0278 & 1.0451 & 1.1 \\
\hline Channel 3 & 1.0067 & 1.0098 & 1.0140 & 1.0200 & 1.0284 & 1.0411 & 1.0623 & 1.1 \\
\hline Channel 4 & 1.0018 & 1.0038 & 1.0070 & 1.0117 & 1.0186 & 1.0290 & 1.0467 & 1.1 \\
\hline Channel 5 & 1.0044 & 1.0075 & 1.0118 & 1.0177 & 1.0257 & 1.0370 & 1.0557 & 1.1 \\
\hline Channel 6 & 1.0085 & 1.0128 & 1.0182 & 1.0250 & 1.0337 & 1.0456 & 1.0650 & 1.1 \\
\hline
\end{tabular}

Table C.2: Table of velocity ratios for channels with boundary layer

\begin{tabular}{|c|c|c|c|c|c|c|c|r|}
\hline Ct & 0.3 & 0.4 & 0.5 & 0.6 & 0.7 & 0.8 & 0.9 & 1 \\
\hline Channel 0 & 0.9996 & 0.9997 & 1.0001 & 1.0014 & 1.0042 & 1.0100 & 1.0224 & 1.0 \\
\hline Channel 1 & 1.0014 & 1.0027 & 1.0051 & 1.0090 & 1.0150 & 1.0242 & 1.0406 & 1.1 \\
\hline Channel 2 & 1.0011 & 1.0016 & 1.0026 & 1.0044 & 1.0077 & 1.0138 & 1.0265 & 1.0 \\
\hline Channel 3 & 1.0009 & 1.0026 & 1.0048 & 1.0078 & 1.0122 & 1.0196 & 1.0338 & 1.0 \\
\hline Channel 4 & 0.9998 & 1.0000 & 1.0008 & 1.0025 & 1.0060 & 1.0127 & 1.0263 & 1.0 \\
\hline Channel 5 & 0.9995 & 0.9998 & 1.0008 & 1.0028 & 1.0066 & 1.0138 & 1.0280 & 1.0 \\
\hline Channel 6 & 0.9972 & 0.9971 & 0.9975 & 0.9986 & 1.0012 & 1.0067 & 1.0188 & 1.0 \\
\hline
\end{tabular}




\section{Appendix D}

\section{MATLAB code for blockage correction}

\%calculates the velocity ratio, uet, and the velocity drop behind the turbine, $\mathrm{u} 2 \mathrm{t}$

\%uses free-stream $\mathrm{Ct}$, forward predicts thrust under blockage

\%variable names follow that found in Bahaj et al. [1]

\%Initialisation of variables

$\mathrm{u} 3 \mathrm{t}=1$;

$\mathrm{u} 13=1$

diff $=1$;

alpha $=1 ;$

$\mathrm{u} 0=0.333 ;$

for $\mathrm{i}=1: \operatorname{size}(\mathrm{Ct}, 2)$

\%first guess of variables

$\mathrm{u} 1 \mathrm{t}=0.5 *(1-\mathrm{sqrt}(1-\mathrm{Ct}(\mathrm{i})))$;

if $\mathrm{Ct}(\mathrm{i})=1$ $\mathrm{ut} 2 \mathrm{~b}(\mathrm{i})=1 / 0.001$;

else $\mathrm{ut} 2 \mathrm{~b}(\mathrm{i})=1 / \operatorname{sqrt}(1-\mathrm{Ct}(\mathrm{i}))$;

end

$\mathrm{f}(\mathrm{i})=4 *(1-\operatorname{sqrt}(1-\mathrm{Ct}(\mathrm{i}))) /(1+\operatorname{sqrt}(1-\mathrm{Ct}(\mathrm{i})))$;

diff $=1$; 
while diff $>0.0001$

$$
\begin{aligned}
& \mathrm{ut} 2(\mathrm{i})=\mathrm{ut} 2 \mathrm{~b}(\mathrm{i}) \text {; } \\
& \mathrm{u} 32=\operatorname{sqrt}\left(\mathrm{f}(\mathrm{i}) * \mathrm{ut} 2(\mathrm{i}) \wedge 2 * \mathrm{u} 1 \mathrm{t}^{\wedge} 2+1\right) ; \\
& \mathrm{u} 12=\left(-1+\operatorname{sqrt}\left(1+\mathrm{B} *\left(\mathrm{u} 32^{\wedge} 2-1\right)\right)\right) /(\mathrm{B} *(\mathrm{u} 32-1)) ; \\
& \mathrm{ut} 2 \mathrm{a}(\mathrm{i})=\mathrm{u} 32-\mathrm{B} * \mathrm{u} 12 *(\mathrm{u} 32-1) \text {; } \\
& \text { diff }=\operatorname{abs}((\text { ut2a(i) }-u t 2(i)) / u t 2(i)) \text {; } \\
& \text { ut } 2 b(\mathrm{i})=0.5 *(\mathrm{ut} 2(\mathrm{i})+\mathrm{ut} 2 \mathrm{a}(\mathrm{i})) \text {; } \\
& \mathrm{u} 3 \mathrm{t}=\mathrm{u} 32 / \mathrm{ut} 2 \mathrm{~b}(\mathrm{i}) \text {; } \\
& \mathrm{u} 13=\mathrm{u} 12 / \mathrm{u} 32 \\
& \mathrm{u} 1 \mathrm{t}=\mathrm{u} 12 / \mathrm{ut} 2 \mathrm{~b}(\mathrm{i}) \text {; }
\end{aligned}
$$

end

$$
\begin{aligned}
& \mathrm{u} 2 \mathrm{t}(\mathrm{i})=1 / \mathrm{ut} 2(\mathrm{i}) ; \\
& \mathrm{u} 1 \mathrm{t}(\mathrm{i})=\mathrm{u} 12 * \mathrm{u} 2 \mathrm{t}(\mathrm{i}) ; \\
& \mathrm{a}(\mathrm{i})=0.5 *(1-\operatorname{sqrt}(1-\mathrm{Ct}(\mathrm{i}))) ; \\
& \mathrm{uet}(\mathrm{i})=\mathrm{u} 1 \mathrm{t}(\mathrm{i}) /(1-\mathrm{a}(\mathrm{i})) ;
\end{aligned}
$$

end 


\section{Related publications}

\section{Journal}

Koh, W.X.M., Ng, E.Y.K.. Effects of Reynolds number and different tip loss models on the accuracy of BEM applied to tidal turbines as compared to experiments. Ocean Engineering 2016;111:104-115

Koh, W.X.M., Ng, E.Y.K.. A CFD study on the performance of a tidal turbine under various flow and blockage conditions. Renewable Energy 2017, In Press

Koh, W.X.M., Ng, E.Y.K.. A CFD study of the performance and wake recovery of a tidal turbine array under various blockage conditions. Submitted to International Journal of Marine Energy 